\title{
MODELS \\ FOR ANALYZING NONLINEARITIES \\ in PRICE TRANSMission
}

\author{
Dissertation \\ zur Erlangung des Doktorgrades \\ der Fakultät für Agrarwissenschaften \\ der Georg-August-Universität Göttingen
}

vorgelegt von

Rico Ihle

geboren in Zschopau

Göttingen, Dezember 2009 
D 7

1. Referent:

Prof. Dr. Stephan von Cramon-Taubadel

2. Korreferent: Prof. Dr. José Maria Gil Roig

Tag der mündlichen Prüfung: 4. Februar 2010 


You always return from a journey as a different man to the one who set off.

(Graham Greene)

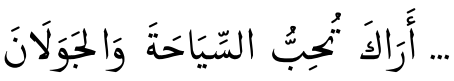

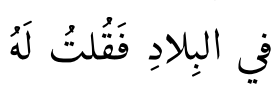

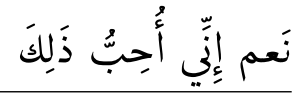
(ابن بطوطة، رحلة) 

"...I see that you are fond of traveling through foreign lands." I replied, "Yes, I am."

(Ibn Battuta, Voyage) 



\section{Danksagung - Acknowledgments}

Hisde, nuu is suuweid. Nuu werdor Gung emohl ferdsch! Nuu kimmor de Dugdurarb abor nich schreim, uhne duß doo e bill, wingsdns e bill Arzgibargsch drinne schdied.

Joa, ze erschdemohl rachd schien Doank an de Luid dehemm, an mei Illorn, mei Schwasdor und un ulle gudn Bekanndn, ze die dar Foodn nuch gehalln hood, ah wenniech inde ledsdn Goahr rachd viel in dor Waldgischichd rimhargirääsd bie un nichesu viel dehemm giwasn bie, wiemor villeichd dehemm sei hädd kinne. Nuijoa, su is dus Lahm ahm. Schien Doank difier, dussor mier'n Riggn freighalln hubd, dussiech ah emohl ewus annorsch ze du hadd uls wie näär de Arb. E bill Oabwachslung dud nischd schoodn! Un ullis, wus mor dehemm gilernd hood, dudor nidsn, ah wenn de idse mid'n Rachnkasdl un nich middor Goobl dei Gald vordiene dusd. Asso: rachd schien Doank!

Nuu muß' abor uff Englisch weddor gieje, duß die annorn miech ah vorschdieje:

The two quotes above illustrate what I consider the last half a decade-but not only that time-to be: a journey. Not only because I traveled, physically, to (or through) 20 countries in this time, but also in the broader and more figurative sense of "journey." Therefore, I would like to take the opportunity now to thank some of the people who guided me on this journey and have thus played a part in making it turn out the way it did.

Those who bear the most guilt for my interest in statistics and econometrics are Professor Stephan von Cramon-Taubadel and Professor Walter Zucchini, who inspired me with their excellent lectures to pursue quantitative methods in my M.Sc. studies. When I was abroad after graduation, I learned about the recently founded Centre for Statistics at Göttingen University, and thus applied from Sana'a, Yemen, for the Ph.D. program "Applied Statistics and Empirical Methods." I am grateful for the support of Professor von Cramon-Taubadel and Professor Zucchini in my application for this program.

I would like to explicitly thank the Centre for Statistics for giving me the chance to study in the program; it has shaped me in a lasting way. Particularly, I would like to thank Professor Manfred Denker for his help in making the start in the program as easy as possible for new students, and for his small bits of wisdom he gave the new Ph.D. students along their way. The courses of Professor Walter Zucchini and Professor Stefan Sperlich familiarized me with the vast and very interesting world of quantitative methods; a warm thank you for this. Because of Professor Edgar Brunner, I became convinced of the usefulness of matrix algebra. Thank you.

Special thanks go to the supervisors of my thesis. Professor Stephan von CramonTaubadel provided much motivation, many helpful comments and a pleasant international working atmosphere at the Lehrstuhl. Many thanks to Professor José-Maria Gil and Professor Bernhard Brümmer for their support and helpful advice which made 
me look at several aspects from new perspectives, and thus enriched the thesis considerably (Muchas gracias und vielen Dank!).

Thanks also to the friends and good colleagues whom I got to know during these last five years in Göttingen. Some of them I would like to name here. First, I shared the office with Rodrigo and Oleg on the $10^{\text {th }}$ floor of the Blauer Turm during this time which was enjoyable to say the least. Moreover, thanks to Arne, Johannes, Paola, Jei, Yesilda, and Rauf, with whom I spent so much time during my first two years in the Ph.D. program. Thank you to my immediate colleagues of the Agrarpolitiklehrstuhl, Dr. Jochen Meyer, Sebastian, Joseph, Karla, and Linde; and my indirect colleagues of the Marktlehrelehrstuhl on the $10^{\text {th }}$ floor, Stefan, Tinoush, and Martin, for the nice time and interesting discussions we had. I would like to also thank the people who co-founded the Arbeitskreis Ökonometrie with me, Julia, Asif, and Yanjie, for their engagement, and the "new" generation of interested and regular participants who have kept it alive since spring 2006. Thanks for the great meetings and critical discussions we had! Also, I have good memories of the engaging talks with and the hospitality of Afsaneh and Arash. And, I will not forget the good time with Ren un de Sochs' $n$ of the Ökonometrielehrstuhl on the $8^{\text {th }}$ floor. I am also thankful for the help of Kristin, Mary, and Stefan for proofreading this thesis.

Last but not least, I do not want to forget the many nice people all over the world whose ways met briefly with mine. Many thanks for their reliability, hospitality, and helpfulness which kept me from getting lost, whether it be in Arabia Felix, the Pamirs, the Rub' al Khali, the Atacama, or the Dasht-e Lut. 




\section{Contents}

List of Figures $\quad$ V

$\begin{array}{lll}\text { List of Tables } & \text { VI }\end{array}$

List of Abbreviations $\quad$ IX

List of Symbols $\quad$ XI

Executive Summary $\quad$ XV

1 Introduction $\quad 1$

1.1 Motivation ........................... 1

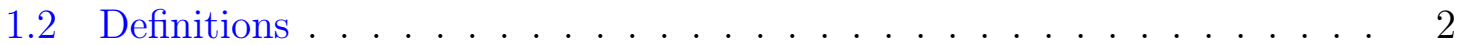

1.3 Vector Error-Correction Model . . . . . . . . . . . . . . . . . 7

1.3 .1 Basic Idea . . . . . . . . . . . . . . . . . . . . 7

1.3.2 Model Structure . . . . . . . . . . . . . . . . . . . . . 8

1.3.3 Estimation . . . . . . . . . . . . . . . . . . . . 12

1.3.4 Interpretation . . . . . . . . . . . . . . . . . 16

1.3.5 Applications . . . . . . . . . . . . . . . . 18

1.4 Nonlinearities in Price Transmission . . . . . . . . . . . . . . . . . 19

1.4.1 The VECM and Nonlinearities . . . . . . . . . . . . . . . 19

1.4.2 Literature Review . . . . . . . . . . . . . . . . . . . 22

2 Models in Detail 25

2.1 Threshold Vector Error-Correction Model . . . . . . . . . . . . . . . . . 25

2.1 .1 Basic Idea . . . . . . . . . . . . . . . . . 25

2.1.2 Model Structure . . . . . . . . . . . . . . . . . . . . . 28

2.1.3 Estimation . . . . . . . . . . . . . . . . 33

2.1.4 Interpretation . . . . . . . . . . . . . . . . . 34

2.1.5 Applications . . . . . . . . . . . . . . . . 34

2.2 Markov-Switching Vector Error-Correction Model . . . . . . . . . . . . 35

2.2 .1 Basic Idea . . . . . . . . . . . . . . . . . . . . . . . . . . . . . . . . .

2.2.2 Model Structure . . . . . . . . . . . . . . . . . . . . . . . . . . . . . . . . . . . . . . . . .

2.2 .3 Estimation . . . . . . . . . . . . . . . 41

2.2 .4 Interpretation . . . . . . . . . . . . . . . . 41

2.2.5 Applications ........................ . . 42 
2.3 Semiparametric Vector Error-Correction Model . . . . . . . . . . . . . 45

2.3 .1 Basic Idea . . . . . . . . . . . . . . . . . . . . . 45

2.3.2 Model Structure . . . . . . . . . . . . . . . . . . 46

2.3 .3 Estimation . . . . . . . . . . . . . . . 46

2.3 .4 Interpretation . . . . . . . . . . . . . . . . . . . . . . . . . . . . . 49

2.3.5 Applications . . . . . . . . . . . . . . . . . . 49

2.4 Parity Bounds Model . . . . . . . . . . . . . . . . . . . 51

2.4 Basic Idea . . . . . . . . . . . . . . . . . . . . . 51

2.4 .2 Model Structure . . . . . . . . . . . . . . . . . 52

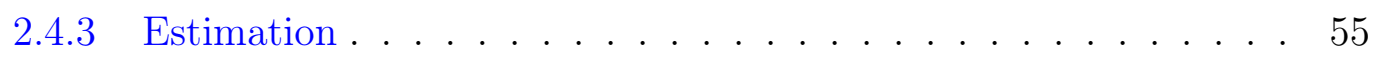

2.4 .4 Interpretation . . . . . . . . . . . . . . . 55

2.4.5 Applications ..................... 57

3 Summary $\quad 59$

3.1 Cointegration Models . . . . . . . . . . . . . . . . . . . . 59

3.2 Parity Bounds Models . . . . . . . . . . . . . . . . 63

3.3 Parity Bounds vs. Cointegration Models . . . . . . . . . . . 67

$\begin{array}{lll}4 \text { Conclusion } & 79\end{array}$

$\begin{array}{ll}\text { Bibliography } & 80\end{array}$

$\begin{array}{ll}\text { Appendices } & 98\end{array}$

Appendix A: Spatial Market Integration in the EU Beef and Veal Sector:

Policy Decoupling and the Outbreak of Blue Tongue Disease . . . . . . 101

Appendix B: Markov-Switching Estimation of Spatial Maize Price Transmis-

sion Processes between Tanzania and Kenya . . . . . . . . . . . . . . 125

Appendix C: Grenzeffekte in der Marktintegration bei Mais in Ostafrika:

Einsichten aus einem semi-parametrischen Regressionsmodell . . . . . . 139

Appendix D: A Comparison of Threshold Cointegration and Markov-Switching

Vector Error-Correction Models in Price Transmission Analysis . . . . . 161

Appendix DI: Literature Review of Applications of the TVECM . . . . 197

Appendix DII: Simulation Study . . . . . . . . . . . . . . . . 201 




\section{List of Figures}

1.1 Nonstationary Process vs. Mean Reversion of a Stationary Process . . . 4

1.2 Stochastic vs. Deterministic Trend . . . . . . . . . . . . . 5

1.3 Example of a VECM . . . . . . . . . . . . . . . . . . 9

1.4 Characteristic Graphs of the VECM . . . . . . . . . . . . . . . . 13

2.1 Example of a Continuous and Symmetric Band-TVECM . . . . . . . . 31

2.2 Example of a Symmetric EQ-TVECM . . . . . . . . . . . . . . . . . . 32

2.3 Characteristic Graphs of a Typical Sym. and Continuous Band-TVECM 33

2.4 Characteristic Graphs of a Typical Symmetric EQ-TVECM . . . . . . . 33

2.5 Estimated Constants of a MSM-VAR and a MSI-VAR Specification . . 39

2.6 Characteristic Graphs of a Typical MSVECM . . . . . . . . . . . . . . 40

2.7 Example of a MSVECM . . . . . . . . . . . . . . . . . . . . . 41

2.8 Characteristic Graphs of a Typical SPVECM . . . . . . . . . . . . . . . 47

2.9 Examples of Unweighted Estimated Mixture Distributions . . . . . . . 54

2.10 Examples of Weighted Estimated Mixture Distributions . . . . . . . . . 55

2.11 Characteristic Graphs of a Typical PBM . . . . . . . . . . . . 56

3.1 Mean and Variance of a Band-TVECM . . . . . . . . . . . . . . . . . 63

3.2 Mean and Variance of an EQ-TVECM . . . . . . . . . . . . . . . 64

3.3 Mean and Variance of a MSVECM . . . . . . . . . . . . . . . . 65

3.4 Distribution of Band-TVECM Realizations (Weak Adjustment) . . . . 73

3.5 Distribution of Band-TVECM Realizations (Strong Adjustment) . . . . 74

3.6 Distribution of EQ-TVECM Realizations (Weak Adjustment) . . . . . 75

3.7 Distribution of EQ-TVECM Realizations (Strong Adjustment) . . . . . 76

3.8 Distribution of MSVECM Realizations (Strong vs. Weak Adjustment) . 77

D.1 Classification of Nonlinear Models after Tong (1990) . . . . . . . . . . . 166

D.2 Transactions Costs and Regime-Dependent PT . . . . . . . . . . . . . 170

D.3 Realization of a TVECM(3) . . . . . . . . . . . . . . . . . 173

D.4 Transition Graph of a Two-State Markov Chain . . . . . . . . . . . . . 180

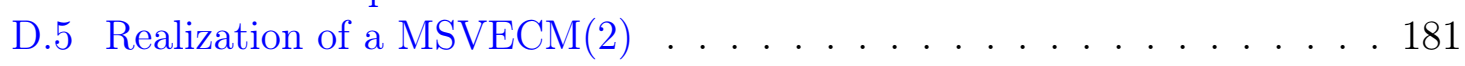

D.6 The Expectation-Maximization Algorithm . . . . . . . . . . . . . . . . 182 



\section{List of Tables}

1.1 Aspects of Nonlinearities . . . . . . . . . . . . . . . . . 19

2.1 Special Cases of the TVECM . . . . . . . . . . . . . . 30

3.1 Correspondence of PBM and Cointegration Terminology . . . . . . . 70

D.1 Estimation Approaches of Selected Publications . . . . . . . . . . . . 174

DI.2 Publications per Type . . . . . . . . . . . . . . . . . . . 197

DI.3 Publications per Research Field . . . . . . . . . . . . . . . 197

DI.4 Publications per Year . . . . . . . . . . . . . . . . . 197

DI.5 Review of Price Transmission Studies in Commodity Markets . . . . . . 200

DII.1Design of the Simulation Study . . . . . . . . . . . . . . . 203 



\section{List of Abbreviations}

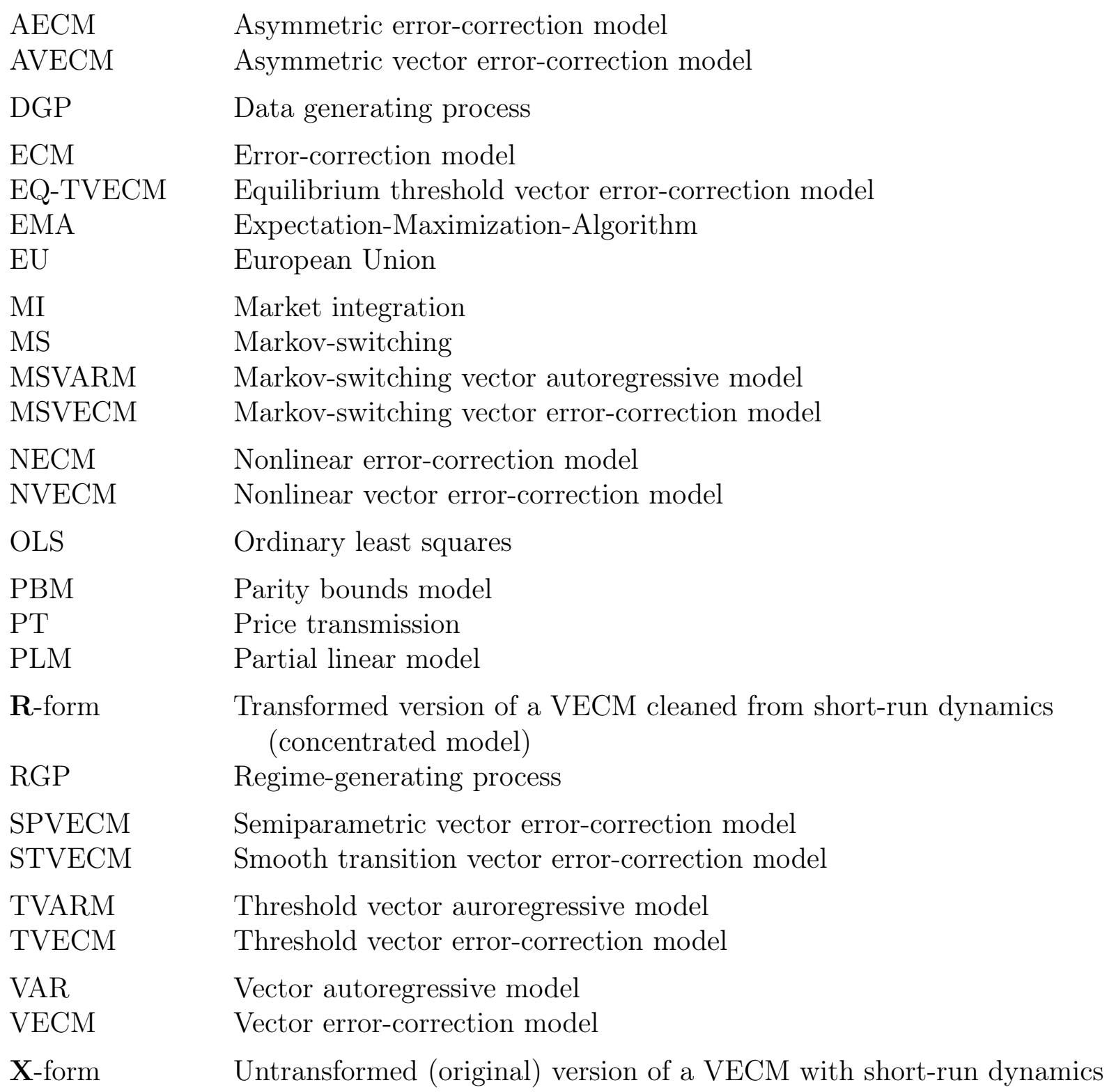





\section{List of Symbols}

\begin{tabular}{|c|c|}
\hline$\alpha$ & $(v \times r)$ matrix containing the loading parameters (adjustment speeds) \\
\hline$\beta$ & $(v \times r)$ matrix containing the cointegration relationships \\
\hline$D_{t}$ & $m$-dimensional vector of deterministic terms \\
\hline$\Delta$ & First difference operator $\left(\Delta y_{t}=y_{t}-y_{t-1}\right)$ \\
\hline$\epsilon$ & Indepedent identically normally distributed error term \\
\hline$F(\bullet)$ & Distribution function \\
\hline$\Phi$ & $(v \times m)$ matrix of coefficients of $D_{t}$ \\
\hline$I(d)$ & Integration of order $d$ \\
\hline iid & Independent and identically distributed \\
\hline$k$ & Number of lags of a vector autoregressive model $(\operatorname{VAR}(k))$ \\
\hline$\kappa$ & Cointegration vector \\
\hline$L$ & Lag/ backshift operator $\left(L y_{t}=y_{t-1}\right)$ \\
\hline$M$ & $\begin{array}{l}\text { Number of states of a Markov-chain } \\
\text { or regimes of a MSVARM/ MSVECM }\end{array}$ \\
\hline$N I D\left(\mu, \sigma^{2}\right)$ & $\begin{array}{l}\text { Independent and identically normal distributed } \\
\text { with mean } \mu \text { and variance } \sigma^{2}\left(\sim N I D\left(\mu, \sigma^{2}\right)\right)\end{array}$ \\
\hline$p$ & $v$-dimensional vector of prices \\
\hline$\Pi=\alpha \beta^{\prime}$ & $\begin{array}{l}(v \times v) \text { matrix containing the loading parameters } \\
\text { and cointegration relationships }\end{array}$ \\
\hline$\Pi_{i}$ & $(v \times v)$ matrix of coefficients of a $\operatorname{VAR}(k), i=1, \ldots, k$ \\
\hline$r$ & Number of cointegration relationships, i.e., $r=\operatorname{rank}(\Pi)$ \\
\hline$R_{0 t}$ & $\begin{array}{l}v \text {-dimensional vector of residuals } \\
\quad \text { from regressing } \Delta p_{t} \text { on } \Delta p_{t-1}, \Delta p_{t-2}, \ldots, \Delta p_{t-k+1}\end{array}$ \\
\hline$R_{1 t}$ & $\begin{array}{l}v \text {-dimensional vector of residuals } \\
\quad \text { from regressing } p_{t-1} \text { on } \Delta p_{t-1}, \Delta p_{t-2}, \ldots, \Delta p_{t-k+1}\end{array}$ \\
\hline$t$ & Time index \\
\hline$T$ & Number of time periods observed (length of time series) \\
\hline$v$ & Number of variables in the system (dimension of $y$ ) \\
\hline$y$ & $v$-dimensional vector of one observation of each of the $v$ variables \\
\hline
\end{tabular}







\section{Executive Summary}

This dissertation aims at providing an overview of various econometric model classes which are suitable for the analysis of price relationships. It discusses a number of models which are suitable for the analysis of price relationships both in the vertical, i.e., of markets along the processing chain, and the horizontal domain, i.e., of markets of one commodity in space. However, the thesis focuses on the latter topic. It provides an in-depth discussion of selected model classes and, in the appendices, three empirical applications of selected models. The applications analyze structural changes in the transmission of European calf prices and regime-dependent behavior of spatial maize price relationships in Eastern Africa. Furthermore, the structure and determinants of the transmission of maize prices in domestic as well as cross-border trade are assessed.

We are not aware of publications in agricultural economics in general and in price transmission analysis in particular which compare various model classes in detail. Hence, this dissertation is a contribution to the literature in three respects. First, it provides an overview of recent developments of the methodology used to analyze price relationships. It focuses on the intuition of four model classes and can thus help to improve the link between econometric theory and the study of empirical questions in applied research in agricultural economics. Second, it serves to relate, to compare and to contrast these classes. Model selection approaches between several competing classes of nonlinear models as well as between nonlinear and linear models based on econometric testing are being developed in the literature. However, the theory is very complicated and thus not many approaches are currently available. Therefore, we believe that a well developed intuition of the fundamental mechanisms governing such models can help the selection of an adequate model class based on the empirical context and economic theory. Thereby, we take an applied point of view and focus on the ideas behind the models, and potentially promising areas of application in applied research. Third, the dissertation provides analyses of the impacts of changing policy environments on the transmission of agricultural commodity prices within the European Union as well as within the East African Community.

CHAPTER 1 briefly presents the background and a number of definitions of properties which characterize the type of data this thesis is focused on, i.e., time series of economic variables. It introduces autoregressive time series models which are particularly useful for the modeling of cointegrated variables, and the relationships between them. It then addresses the underlying idea, model structure, estimation, interpretation, and applications of the traditional vector error-correction model. First, the potential role of disequilibrium besides the usual lag-structure dynamics on the development of economic variables along time is discussed. This idea is then formalized in an autoregressive model and illustrated by suitable graphs. The Frisch-Waugh-Lovell Theorem 
is presented in order to give the background of a particular version of the model, the so-called $\mathbf{R}$ (educed)-form. This version is cleaned from autoregressive dynamics and, thus, contains variables only quantifying the dynamics of the equilibrium restoring forces. We present a number of graphs which provide a schematic summary of key characteristics of the model. The estimation section first discusses various estimation approaches of the long-run relationship and, second, elaborates Johansen's reduced rank regression in some detail. Afterwards, we define our understanding of core concepts regarding the interpretation of cointegration models in the context of spatial price analysis and refer to selected literature for applications. A discussion of different forms of nonlinearities in price transmission follows. This serves to confine the focus of this thesis which lies in nonlinearities in short-run price transmission. Such nonlinearities are caused by nonconstant loading coefficients which quantify the speed with which deviations from the equilibrium are adjusted. Parametric functions, most often in the form of regime-dependent models, and nonparametric functions might be suitable choices for modeling them. Moreover, the notion of the regime-generating process is introduced. The chapter closes with a brief literature review of the econometric theory of nonlinear vector error-correction models and of applications of the latter modeling strategy via nonparametric functions.

CHAPTER 2 presents the four model classes in detail. The classes of the threshold vector error-correction model, the Markov-switching vector error-correction model and the semiparametric vector error-correction model belong to the family of cointegration models. The parity bounds model represents a class of the family of mixture distribution models. The chapter follows the same structure as the presentation of the vector error-correction model does by addressing the underlying idea, the formal structure, estimation, interpretation, and applications of each class. It partly draws on Appendix D and provides mostly additional thoughts instead of repeating the content of this appendix. We take an applied view and focus on the intuition behind and the interpretation of the model classes.

Chapter 3 provides a critical summary of the outlined models. It first discusses a number of problems of the three presented classes of cointegration models. Afterwards, it elaborates on properties of suitable regime-generating processes. This is followed by a critical discussion of the parity bounds model. The chapter closes with a critical comparison of both model families highlighting the advantages and drawbacks of each. Furthermore, similarities and differences between the thresholds and the parity bounds models are addressed. The distributions of the underlying data which are implied by selected versions of cointegration models are contrasted to the distributional assumptions of the parity bounds model. CHAPTER 4 concludes the dissertation.

ApPENDIX A studies spatial price transmission between European calf markets. It focuses on the effects of the decoupling of agricultural support from production and of movement restrictions, which resulted from the outbreak of the Blue Tongue disease in central Europe. The 2003 reforms of the Common Agricultural Policy of the European 
Union allowed for discretionary implementation among member states. Discretion was allowed with respect to the timing and the degree of decoupling of policy support. Differences among member states were particularly apparent in the European beef and veal sector. Using weekly data from 2003 to 2009, we assess the consequences of different national implementation strategies of the reforms on price transmission for young calves, which are intensively traded in the European Union. Time series properties are analyzed by employing a range unit-root test and a multivariate cointegration model. We find that the calf markets in Germany, France, the Netherlands and Spain are integrated and tightly interrelated and can thus be considered to belong to a common market. This conclusion is supported by the estimated persistence profiles which track the absorption of shocks by the system of prices. We also find strong statistical support for the hypothesis that decoupling reduced calf price levels. Using counterfactual scenarios, evidence of the effects of different policy choices on the price system is obtained. Additionally, our results indicate that the outbreak of the Blue Tongue disease induced a structural change in parts of the EU calf market.

APPENDIX B gives an overview of the Markov-switching vector autoregressive class of non-linear time series models which can be used to analyze recurring discrete structural breaks in time series. Most empirical applications of the model to date have focused on the business cycle and financial markets, but we see potential for this model class in agricultural economics, for example in price transmission analysis. We first provide an overview of the model framework. We then present an illustrative application to the analysis of spatial maize prices between Tanzania and Kenya. We employ a Markov-switching vector autoregressive model to assess regimes in maize price margins between each of four regions in Tanzania and Nairobi, the capital of Kenya. We conjecture that temporarily implemented Tanzanian export bans might have caused the alternating phases of high and low margins which characterize the data. However, the available information on trade restrictions seems not to be complete. Moreover, we lack information on the effectiveness of their implementation. We analyze monthly prices from 2000 to the end of 2008. We identify five periods of increased margins which last from four to seventeen months and partly correspond to the timings of export restrictions reported by the literature. During these periods, the margins' averages are more than doubled and their standard errors considerably reduced in comparison to periods of low margins.

APPENDIX $\mathrm{C}$ analyzes factors which influence the magnitude of price transmission in domestic and cross-border trade of East African maize markets. There is an extensive literature on distance and border effects in trade, but little attention has been paid to the impact of distance and borders on spatial price transmission. We analyze distance and border effects in maize price transmission between Kenya, Tanzania and Uganda. Using monthly data from January 2000 to October 2008, maize price transmission is measured for 85 market pairs within and between these countries. The magnitude of price transmission between market pairs is found to vary systematically with distance and the presence or lack of a national border between a market pair. This analysis 
extends the border effects literature in three ways. First, price transmission rather than trade flows or price variability is analyzed. Second, the impact of distance on price transmission is shown to be nonlinear, and is modeled using a semi-parametric partially linear regression model. Third, strong evidence is found that border effects are heterogeneous; the Tanzanian border has a significant negative impact on price transmission, while the Ugandan border has no effect. The results suggest that Tanzania is a relatively isolated and internally fragmented island within the East African maize market. Price transmission between Nairobi and other markets is strong, which confirms the role that Nairobi plays as a hub in East African trade in general and East African maize markets in particular.

APPENDIX D compares two regime-dependent econometric models for price transmission analysis, namely the threshold vector error-correction model and Markovswitching vector error-correction model. We first provide a detailed characterization of each of the models, which is followed by a comprehensive comparison. We find that the assumptions regarding the nature of their regime-switching mechanisms are fundamentally different, so that each model is suitable for a certain type of nonlinear price transmission. Furthermore, we conduct a Monte Carlo experiment in order to study the performance of the estimation techniques of both models for simulated data. We find that both models are adequate for studying price transmission since their characteristics match the underlying economic theory and hence allow for an easy interpretation. Nevertheless, the results of the corresponding estimation techniques do not reproduce the true parameters and are not robust against nuisance parameters. The comparison is supplemented by a review of empirical studies of nonlinear price transmission which focuses on the class of threshold vector error-correction models which is the most popular nonlinear model in the analysis of price transmission to date. 




\section{Introduction}

This chapter briefly addresses the economic background of this dissertation. It develops the underlying statistical concepts and confines the topic of the thesis from related areas.

\subsection{Motivation}

Knowledge on the dynamics and interrelationships of commodity markets across space (horizontal) and along the processing chain (vertical) is relevant in many circumstances. Fackler and Goodwin (2001) mention, for example, the definition of the size of a market in space, antitrust regulation, or trade modeling. Both economic agents and political decision makers can profit from insights into these questions either in order to assess past actions and decisions or to derive guidelines for future action. Furthermore, scientists and international organizations, such as the Worldbank or FAO, share a particular interest in the analysis of markets for staple foods in developing countries; see, e.g., Rapsomanikis et al. (2003) or Conforti (2004).

Analyses of this topic can have different focuses. Partly due to the fact that price data of commodity trade represents the most easily available data in many cases, considerable interest focuses on this type of data. An extensive literature focused on horizontal and vertical price analyses exists, e.g., in agricultural economics research, which is referred to in this dissertation as price transmission (PT) analysis. This dissertation contributes to this line of research in which it focuses on spatial price analysis. It discusses selected recent models in detail and thus provides an overview of recent developments. In doing so, it takes an applied research point of view and focuses on the intuition behind the models and their interpretation. Furthermore, it includes three empirical analyses of price relationships in European calf and East African maize markets. Therefore, it contributes to the literature by reviewing central properties of several model classes and by studying the effects of differing and changing policy environments on spatial price relationships. It discusses potential applications of each model class in PT analysis and, sporadically, in general applied research in agricultural economics. For selected models, a critique of the methodology is provided and improvements are suggested.

Often, models in PT analysis implicitly assume that the mechanisms which generated the observed data did not change during the period studied. However, this represents 
a strong assumption since it implies that the behavior of economic agents remains unaffected by exogenous events. This dissertation focuses on models which do not make this assumption. It deals with models accounting for nonlinearities in PT analysis by allowing the underlying economic processes to vary in response to incentives or exogenous events.

Prices of a homogeneous commodity on spatially separated markets might react to each other due to the flow of information, e.g., on past price developments, or the physical flow of commodities via trade. A notion closely connected to this issue is spatial arbitrage understood as the exchange of commodities with the objective of taking advantage of price differences (Shepherd, 1997). Furthermore, the movement, i.e., the transaction of the commodity across space, is connected with a number of costs. Transaction costs encompass all expenses which have to be covered to conduct trade, for example, freight costs, duties, costs for insurance etc.; see, e.g., Barrett (2001) for an in-depth discussion.

The empirical analysis of the interrelationships of prices of a homogeneous commodity across space can be approached with a number of methodologies. Among recently developed methods are cointegration models and parity bounds models. Tastan (2003) and Fackler and Tastan (2008) suggest estimation methods of indirect inference. Furthermore, directed acyclic graphs may be used; see, e.g., Tastan (2003). Among these, the first two methods are most popular in current research. Therefore, we focus on the model families of cointegration and parity bounds models. The family of cointegration models encompasses various model classes of which we discuss selected ones in this dissertation. Approaches such as the seemingly unrelated error-correction model of Thompson et al. (2002) or the smooth transition vector error-correction model (see, e.g., Mainardi, 2001; Serra et al., 2008; or Ubilava and Holt, 2009) are not dealt with here.

\subsection{Definitions}

This dissertation deals with multivariate time series models, that is, models for at least two variables observed at the same points in time. The observations of period $t$ are summarized into a $v$-dimensional vector $y_{t}$

$$
y_{t}=\left(y_{1 t}, \ldots, y_{v t}\right)^{\prime}
$$

which is observed at time points $t=1, \ldots, T$. In PT analysis, this is a vector of prices either observed for a homogeneous commodity at different points in space (horizontal PT) or for different levels of the processing chain (vertical PT). Such a multivariate time series as an entity represents one realization of a stochastic process which is an ordered series of random variables. The process will be referred to in the remainder of this work as data generating process (DGP). In the following, we state the definitions of several fundamental properties of processes relevant in the context of PT analysis. 
Definition 1 (Weak Stationarity). The stochastic process $\left\{y_{t}\right\}, t=\ldots,-2,-1,0,1,2, \ldots$ is said to be weakly stationary if

$$
\begin{aligned}
E\left[y_{t}\right] & =-\infty<\mu<\infty & & \forall t, \\
E\left[\left(y_{t}-\mu\right)\left(y_{t+h}-\mu\right)\right] & =\Sigma_{h}<\infty & & \forall t \text { and } h=0,1,2, \ldots
\end{aligned}
$$

This property of time-invariant first and second moments is also termed covariance stationarity. It means that the mean, variance and covariance of the process remain constant over time. ${ }^{1}$

Definition 2 (Strict Stationarity). The stochastic process $\left\{y_{t}\right\}, t=\ldots,-2,-1,0,1,2, \ldots$ is said to be strictly stationary if

$$
F\left(y_{t_{1}}, \ldots, y_{t_{k}}\right)=F\left(y_{t_{1}+h}, \ldots, y_{t_{k}+h}\right) \text { for } h=\ldots,-2,-1,0,1,2, \ldots
$$

where $F(\bullet)$ denotes the distribution function.

A stationary process according to Definition 1 is characterized by, among other properties, a constant mean and a constant variance which leads to a process that exhibits pronounced mean reversion. That is, it shows a strong tendency to revert towards its constant mean and to cross it frequently. It does not drift too far apart from this value, in contrast to a nonstationary process, which may extensively and unpredictably wander around (Figure 1.1). This property of a stationary process is central to the interpretation of the models discussed in detail below.

An alternative perspective particularly useful in this context is available in the so-called stochastic trends, which are the source for the nonstable behavior of nonstationary processes (see, for example, Enders 2004, ch. 4.2; or Juselius 2008, ch. 2). A stochastic trend is characterized by a nonconstant slope which is stochastically changing from period to period. An example of a stochastic trend is contrasted to the constant slope of a deterministic trend in Figure 1.2 below.

Definition 3 (Integration). A process $y_{t}$ is called integrated of order $d$, abbreviated as $y_{t} \sim I(d)$, if $\Delta^{d} y_{t}=(1-L)^{d} y_{t}$ is stationary, where Lyt $\equiv y_{t-1}$ denotes the lag or backshift operator.

According to this definition, a stationary process is integrated of order zero which is denoted as $I(0)$. Processes with $d \geq 1$ are referred to as nonstationary processes. One type of a nonstationary process is the unit root process which is defined as a nonstationary process whose characteristic function has roots which equal unity. ${ }^{2}$ In

1 We use the short-hand term stationarity to refer to the concept of weak stationarity because it suffices for the following discussions.

2 Such roots may be real or complex. If all roots lie inside the unit circle, i.e., the absolute values of 


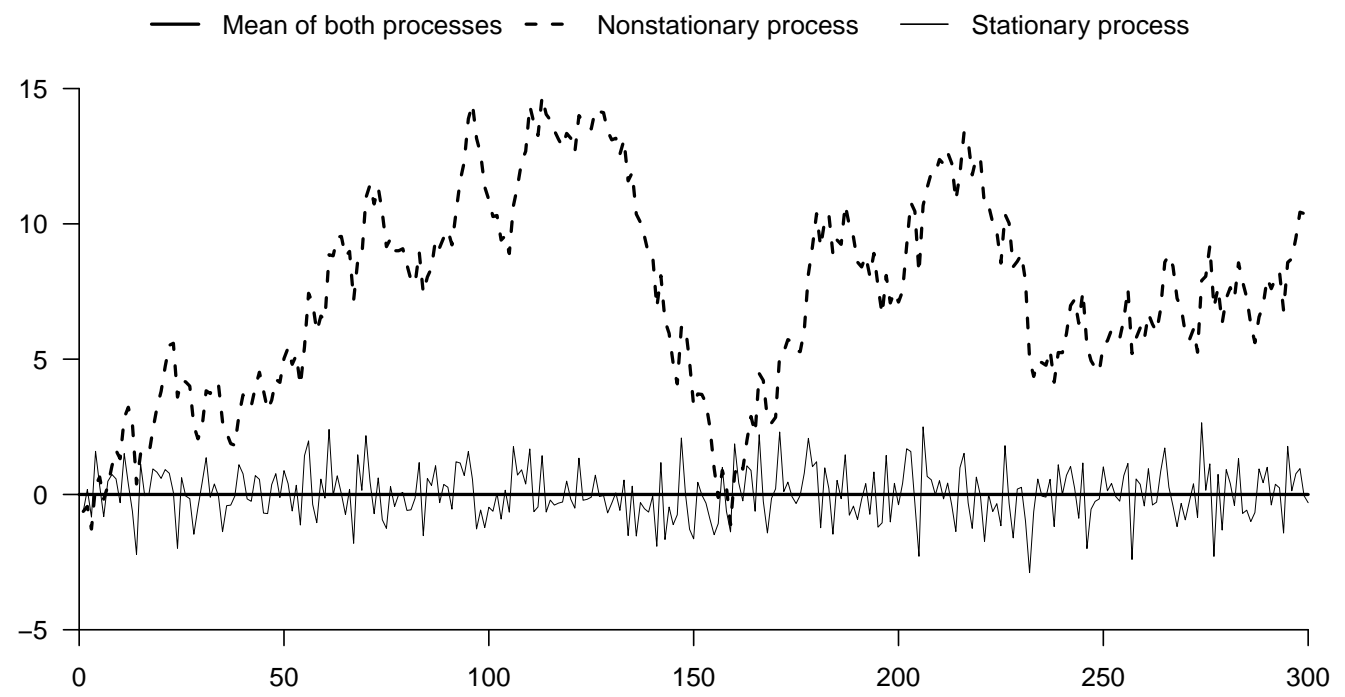

Figure 1.1: Nonstationary Process vs. Mean Reversion of a Stationary Process

this thesis, we only consider processes with at most one unit root, that is, $I(1)$ processes for which $\Delta y_{t}=(1-L) y_{t}=y_{t}-y_{t-1} \sim I(0)$.

Definition 4 (Cointegration). An $I(d)$ process $y_{t}$ is called cointegrated of orders $(d, b)$, that is $y_{t} \sim C I(d, b)$, with cointegrating vector $\kappa$ if $\kappa^{\prime} y_{t}$ is integrated of order $d$-b, that is, $\kappa^{\prime} y_{t} \sim I(d-b)$ where $b=1, \ldots, d ; d=1,2, \ldots ; \kappa \neq 0$.

Since this thesis focuses on processes which are at most $I(1)$, we mean, when using the term cointegration, linear combinations of stochastic processes which are stationary $(I(0)) .^{3}$

The stochastic trend perspective on cointegration has a very intuitive appeal. Variables can only be cointegrated if they possess at least one common stochastic trend. The linear combinations via $\kappa$ lead to the cancellation of the common stochastic trends between certain subsets of the multivariate processes so that the resulting combinations are no longer driven by stochastic trends and are thus stationary. However, the multivariate system as an entity will be driven by at least one stochastic trend if $y_{t} \sim I(1)$.

all real roots and the moduli of all complex roots are smaller than one, then the process is stationary which means that it shows exponentially declining behavior. Complex roots yield cyclical behavior. If a real root is lying on the unit circle, i.e., its absolute value equals unity, then the resulting process is nonstationary. A modulus of one of a complex root yields seasonal nonstationary behavior. Both cases are called a unit root. The number of unit roots of the process corresponds to the order of integration $d$. Roots outside the unit circle correspond to explosive behavior (Juselius, 2008, p. 49 ff). We follow Johansen (1995, Assumption 1, p. 14) and exclude explosive and seasonal roots since such processes are beyond the scope of this work.

3 We follow Lütkepohl and Krätzig (2004, p. 89) and allow for $I(0)$ variables in cointegration relationships. 


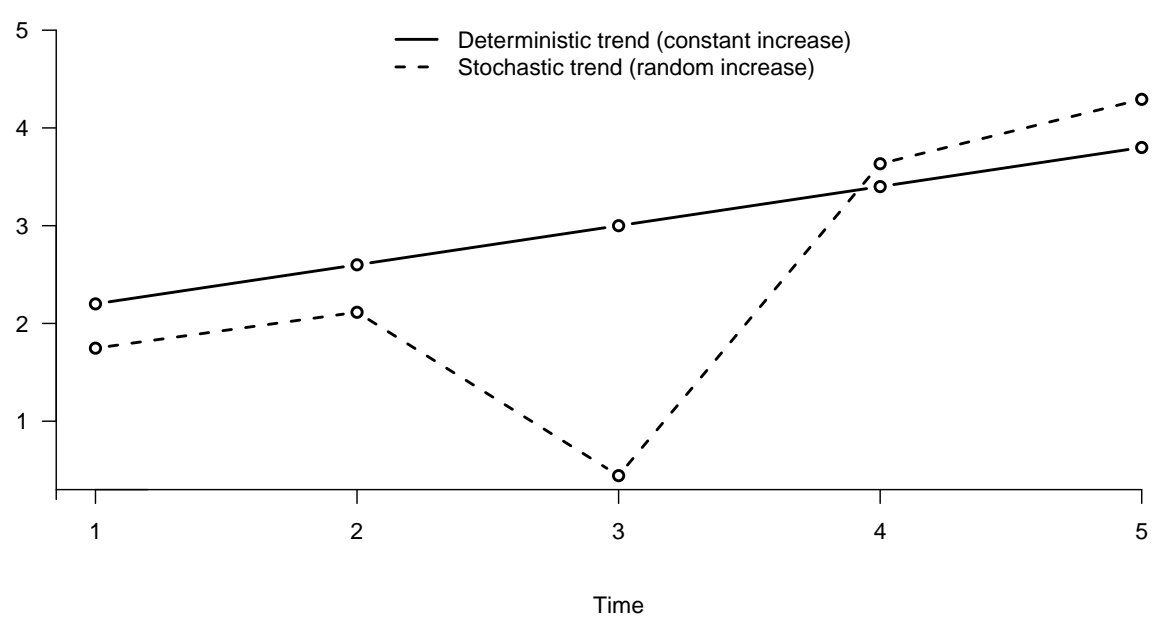

Figure 1.2: Stochastic vs. Deterministic Trend

\section{The Basic Model}

The basic model class considered here is the vector autoregressive model (VAR). In general, the model has $k$ lags:

$$
y_{t}=\Pi_{1} y_{t-1}+\cdots+\Pi_{k} y_{t-k}+\epsilon_{t} .
$$

It is said to be of order $k$, denoted as $\operatorname{VAR}(k) .{ }^{4}$ The $k$ presample values $y_{-k+1}, \ldots, y_{0}$ are assumed to be fixed. The errors $\epsilon_{t}$ are assumed to be independent and identically distributed $(i i d)$ with a multivariate normal distribution $N_{v}(0, \Omega) . \Pi_{i}, i=1, \ldots, k$ denotes $(v \times v)$ coefficient matrices.

Although model (1.2) is apt to represent both stationary and nonstationary processes, in most cases it is not the most convenient formulation due to a number of reasons. It can be repara-metrized into the so-called vector error-correction model (VECM) which is identical to the VAR representation (Juselius, 2008, p. 60-61). The VECM parametrization eases the multicollinearity problem of the VAR formulation. Most importantly, it enables a more intuitive interpretation of the estimated parameters because it is able to distinguish between long-run and short-run effects due to an orthogonalization of the variables. Thus the $\operatorname{VECM}(k-1)$ becomes

$$
\Delta y_{t}=\Pi y_{t-1}+\Gamma_{1} \Delta y_{t-1}+\cdots+\Gamma_{k-1} \Delta y_{t-k+1}+\epsilon_{t}
$$

The $(v \times v)$ matrix $\Pi=-\left(I-\Pi_{1}-\cdots-\Pi_{k}\right)$ contains the influence of the levels of the variables of the previous period. The $(v \times v)$ matrices $\Gamma_{i}=-\left(\Pi_{i+1}+\cdots+\Pi_{k}\right), i=$

\footnotetext{
${ }^{4}$ Model (1.2) can also be augmented by $\Phi D_{t}$, where $D_{t}$ is a vector of $m$ deterministic terms such as a constant, a linear trend, seasonal or interventional dummies. However, we do not consider this term here because the focus of this work lies not on the role of deterministic variables in this model. The methods discussed later on are easily adapted for such terms. Detailed accounts can be found, among others, in Johansen (1995) and Juselius (2008).
} 
$1, \ldots, k-1$ quantify the influences of lagged price changes $\Delta y_{t-i}, i=1, \ldots, k$ and are usually interpreted as short-run dynamics. Johansen (1995, Lemma 4.1, p. 47) shows that the inverse characteristic polynomial of the process described in (1.3) can be written as

$$
A(z)=-\Pi+(\Lambda+\Pi)(1-z)+A^{*}(z)(1-z)^{2}
$$

where $A^{*}(z)$ is also a polynomial and $\Lambda=I-\sum_{i=1}^{k-1} \Gamma_{i}$. The process is stationary if $A(z)$ has no roots with modulus $\leq 1$. Since the case of nonstationary variables $y_{t} \sim I(1)$ is of particular interest for applied analysis, roots for $z=1$ are allowed for. For unit root processes, that is for the case $z=1$, the polynomial (1.4) becomes $A(1)=-\Pi$. The characteristic polynomial is then calculated as

$$
\operatorname{det}(A(1))=\operatorname{det}(-\Pi)=-\operatorname{det}(\Pi)=0 .
$$

Consequently, $\Pi$ is a singular matrix if $y_{t}$ is a unit-root process. That is, the matrix $\Pi$ is not of full rank. Its rank $r$ is smaller than $v(\operatorname{rank}(\Pi)=r<v)$ which is the number of observed variables. The Granger Representation Theorem (Engle and Granger, 1987) states that in such a case the matrix $\Pi$ can be factorized into

$$
\Pi=\alpha \beta^{\prime}
$$

for some $\alpha$ and $\beta$ matrices of dimension $(v \times r) .^{5}$ That is, $\Pi$ has to be of reduced rank $r$ so that (1.3) becomes

$$
\Delta y_{t}=\alpha \beta^{\prime} y_{t-1}+\Gamma_{1} \Delta y_{t-1}+\cdots+\Gamma_{k-1} \Delta y_{t-k+1}+\epsilon_{t}
$$

This point can also be seen from a more intuitive perspective. We allow at most for $I(1)$ processes. The first differences $\Delta y_{t}$ of such processes are then $I(0)$, which means that the difference terms $\Delta y_{t-i}, i=0, \ldots, k-1$ on both sides are stationary. Only the term $\alpha \beta^{\prime} y_{t-1}$ contains nonstationary variables. But since a stationary term on the left-hand side of the equation can never equal the sum of a nonstationary and a stationary term on the right-hand side, $\alpha \beta^{\prime} y_{t-1}$ has to be stationary as well, which implies that $\Pi$ must be of reduced rank. Furthermore, since an I(0) term premultiplied by some matrix remains stationary (Lütkepohl and Krätzig, 2004, p. 89), $\left(\alpha^{\prime} \alpha\right)^{-1} \alpha^{\prime} \alpha \beta^{\prime} y_{t-1}=\beta^{\prime} y_{t-1}$ has to be stationary as well. Hence, $\beta^{\prime} y_{t-1}$ is integrated of order zero while $y_{t-1}$ is integrated of order one which illustrates that $\beta^{\prime} y_{t-1}$ is a $(r \times 1)$ vector of $r$ cointegration relations as defined in Definition 4 . That is, $\beta$ quantifies the linear combinations of the variables $y$ which lead to the elimination of the stochastic trends common to the respective sets of variables. ${ }^{6}$ Hence, the matrix is called the cointegration matrix, and $r=\operatorname{rank}(\Pi)$ the cointegration rank of the system. The matrix $\alpha$ is referred to as the loading matrix. ${ }^{7}$

\footnotetext{
${ }^{5}$ Neither matrix is unique, since for any nonsingular matrix $B$ of dimension $(r \times r)$ it holds that $\Pi=\alpha \beta^{\prime}=\alpha B\left(\beta B^{\prime-1}\right)^{\prime}$, i.e., many linear transformations of them exist.

${ }^{6}$ For the relationship between stochastic trends and cointegration relations, see footnote 10, p. 8 .

7 The parameters of this matrix have to satisfy certain stability conditions with respect to sign and magnitude to ensure that a stable relationship is indeed achieved. Johansen (1995, Theorem 4.2, p.
} 


\subsection{Vector Error-Correction Model}

\subsubsection{Basic Idea}

In their seminal paper, Engle and Granger (1987, p. 251) characterize the fundamental idea of error-correction models as

"equilibrium relationships, where equilibrium is a stationary point characterized by forces which tend to push the economy back toward equilibrium whenever it moves away."

for which Clive W.J. Granger received the 2003 Nobel prize in economics. The complexity of economic systems leads to complicated interdependencies and manifold sources of exogenous shocks potentially impacting the variables studied. Due to the infinitively large set of possible combinations of magnitudes, timings, sources and interactions of such shocks, economic equilibrium relationships which are established theoretically can rarely - if at all - be observed in economic systems. What usually is observed are temporary deviations from equilibria which Engle and Granger call equilibrium errors. Hence, the behavior of economic variables along time which are tied together by equilibria depends not only on the past movements of these variables but also on the magnitude of disequilibrium and the economic forces which try to bring the variables involved back to their equilibrium values. A crucial factor determining the behavior of such variables would be omitted if the influence of the usually existing disequilibrium on the variables were not regarded. Since economic equilibrium-restoring forces try to correct the equilibrium errors, this phenomenon is termed error-correcting behavior. Hence, the behavior of a set of economic variables, say prices, which are tied to each other by at least one equilibrium relationship can be formulated as

$$
\begin{aligned}
\text { current price movement } & =\text { error }- \text { correction }+n(\text { past price movement }) \\
& =g(\text { past equilibrium errors })+n(\text { past price movement })
\end{aligned}
$$

where $g(\bullet)$ and $n(\bullet)$ are linear functions, typically coefficients which are multiplicatively connected with the respective variables, i.e., the $\alpha$ and $\Gamma_{i}$ coefficients in (1.6).

Early versions of this model class were "formulated as the response of one variable, the dependent variable, to shocks of another, the independent variable" (Engle and Granger, 1987, p. 259). The vector version of the model, that is, the VECM, however, extends the approach to more than one equation with at least two variables and allows for complex interdependencies between the variables by treating all of them

$49 \mathrm{ff})$ provides the following condition which has to be checked in case the loading coefficients have the wrong sign since their effect is not easily assessed. If $\alpha_{\perp}^{\prime} \Gamma \beta_{\perp}$, where $\alpha^{\prime} \alpha_{\perp}=0, \beta^{\prime} \beta_{\perp}=0$, is of full rank then cointegration takes place for the respective $\beta$ even though the variables themselves might be explosive. 
as endogenous. ${ }^{8}$ This includes the possibility of various equilibria between subsets of them. The importance of the Granger Representation Theorem lies in proving the equivalence of cointegration and error-correction. Hence cointegrated variables can be seen as the results of economic equilibria and exhibit error-correction behavior. On the other hand, variables which are generated according to a (vector) error-correction process are cointegrated. Here, the parallel to the concept of equilibrium in economics becomes apparent. An equilibrium defines a stable relationship according to which economic agents act. Due to the complexity of the economic system, stochastic influences usually do not permit the attainment of the equilibrium in practice. Whenever the economic system is not in equilibrium, incentives are created for economic agents to react - with the result that the system tends back towards its equilibrium state.

One can imagine a number of areas in which the notion of economic equilibria and the interdependencies of the variables tied to it are of interest. Examples are demographics, voting behavior, trade and price analysis as mentioned in Engle and Granger (1987, p. 254): "The idea is simply that a proportion of the disequilibrium from one period is corrected in the next period. For example, the change in price in one period may depend upon the degree of excess demand in the previous period." Hence, this methodology seems suitable to the study of price dynamics and price interrelationships, in particular for PT analysis.

\subsubsection{Model Structure}

The VECM is formulated as in (1.6) in the following way:

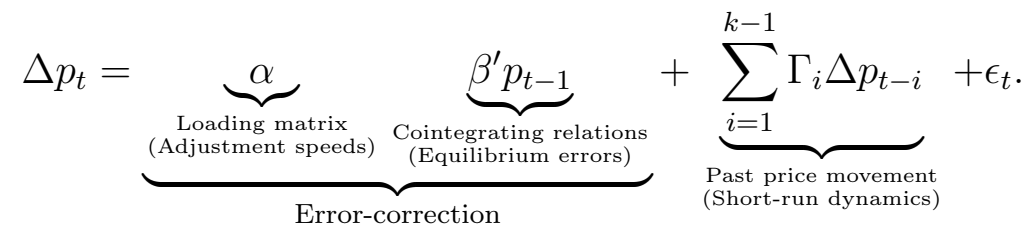

As mentioned above, $\Gamma_{i}, i=1, \ldots, k-1$, denote the parameters quantifying the shortrun dynamics, that is, the influences of past price changes $\Delta p_{t-i}, i=1, \ldots, k-1$ on current price changes. ${ }^{9} \beta$ denotes the cointegration vector, i.e., the weights which lead to the cancellation of the stochastic trends among the sets of cointegrated variables. ${ }^{10}$ Each linear combination via the columns of $\beta$ yields the difference between

\footnotetext{
8 Of course, such a model can be augmented by variables which influence some of the endogenous ones but are not influenced by them. This issue is beyond the scope of this work; see, e.g., Garratt et al. (2006) and references therein.

9 The dimensions of the matrices and vectors involved correspond to the ones of equations (1.3) and (1.6). Note that the constant may either be restricted to the cointegration space, that is, included in the cointegration relations as in (1.8), i.e., $p_{t}=\left(\text { const }, p_{1 t}, \ldots, p_{v t}\right)^{\prime}$, or may be modeled outside the cointegration relations so that $p_{t}=\left(p_{1 t}, \ldots, p_{v t}\right)^{\prime}$.

10 This points to the duality between stochastic trends and cointegration relationships. In a system of $v$ variables and $r$ cointegration relationships, there are $v-r$ remaining stochastic trends which do not cancel and drive the system. These are also called common trends; see, e.g., Johansen (1995, ch. 3), Juselius (2008, ch. 5), and Enders (2004, ch. 6) for detailed discussions and illustrating graphs.
} 
each (hypothetical) equilibrium price and the respective observed price, i.e., the residual. Thus, the term $\beta^{\prime} p_{t-1}=e^{e} t_{t-1}$ also quantifies the equilibrium errors of each cointegration relationship for each time point $t, t=1, \ldots, T . \alpha$ denotes the so-called loading matrix which contains the magnitudes and directions of the responses of $\Delta p_{t}$ to the equilibrium errors $\beta^{\prime} p_{t-1}$. It quantifies the rates or the relative speeds at which the adjustments of the equilibrium errors occurs. Its elements typically have values between -1 and $1 .^{11}$

An example of a typical VECM of two variables is depicted in Figure 1.3. In the upper left panel, the close co-movement of the two price series becomes apparent. Furthermore, the common stochastic trend which drives the cointegrated system, that is, which causes the system to move upwards and downwards, is plotted as a dashed line. In the upper right panel, the mean reversion behavior of the stationary equilibrium error ect $t_{t}$ can be seen. The green line indicates whether the model parameters are constant or show regime-dependent behavior, which will be addressed in more detail below. Clearly, the parameters of the VECM are constant. The lower two panels show the densities of the generated prices and equilibrium errors.
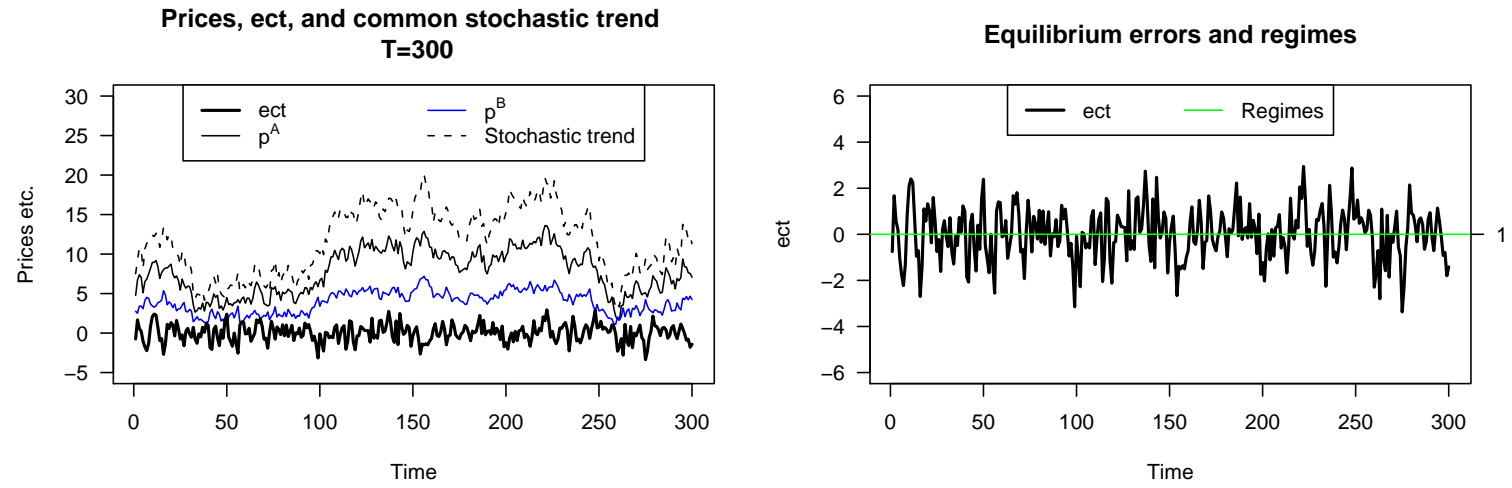

Densities of prices
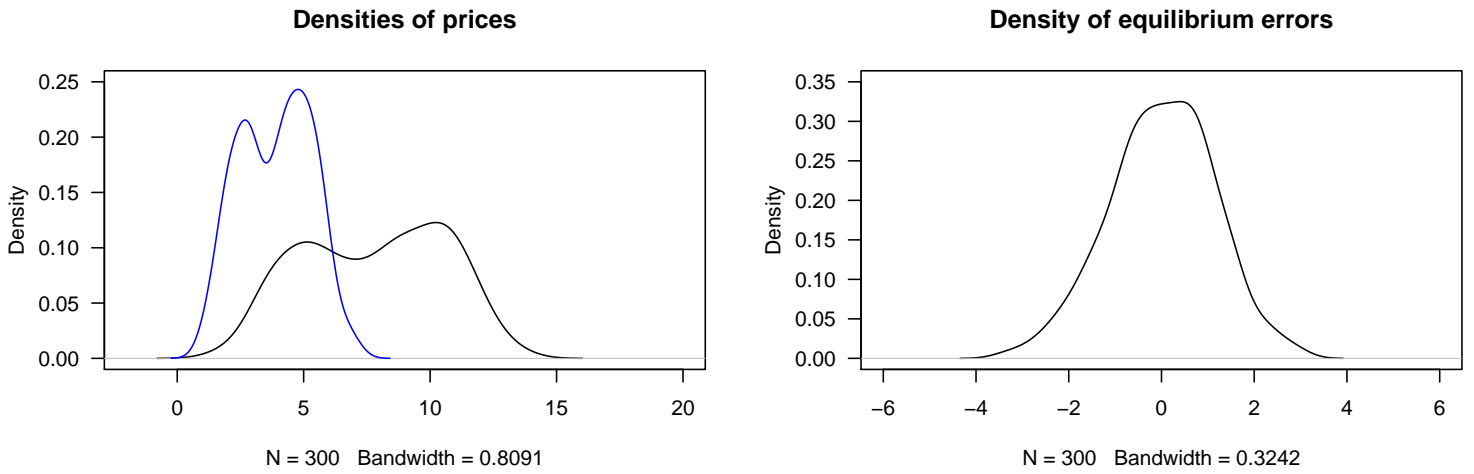

Note: The model is generated according to equations (1) and (2) in Balke and Fomby (1997, p. 629). The parameters are set in order to obtain a reasonable model which potentially could be observed in practice, particularly regarding the adjustment speeds $\alpha$. The simulation uses the parameters $\alpha=-2, \rho=0.4, \beta=1$, and $\epsilon_{t}, \eta_{t} \sim N I D(0,1)$ (notation of Balke and Fomby). Hence, the resulting parameters of model (1.8) are $\alpha=(-0.20 .2)^{\top}, \beta=(1-2)^{\top}$, and $k=1$, i.e., no short-run autoregressive dynamics are existing $\left(\Gamma_{i}=0\right)$.

Figure 1.3: Example of a VECM

\footnotetext{
${ }^{11}$ For the condition indicating the stability of the relationship, see footnote 7, p. 6 .
} 
At this point, we introduce a related specification, i.e., a transformation of this model which will be referred to several times in the remainder of this thesis. This specification concentrates out, or "cleans," the model from the influence of the short-run dynamics $\Gamma_{i}$ which are usually not central for interpretation anyhow. Moreover, it is the key to Johansen's maximum likelihood approach (Johansen, 1988, 1991) and will turn out to be useful in the dissertation later on. ${ }^{12}$ The theoretical basis of this transformation is the Frisch-Waugh-Lovell Theorem (Frisch and Waugh, 1933; Lovell, 1963).

Theorem 1 (Frisch-Waugh-Lovell Theorem). Let $y=X \beta+u$ be a linear model where $X$ and $\beta$ are of adequate size and can be partitioned into $\left(X_{1} X_{2}\right)$ and $\left(\beta_{1}^{\prime} \beta_{2}^{\prime}\right)^{\prime}$, respectively, so that

$$
y=X_{1} \beta_{1}+X_{2} \beta_{2}+u .
$$

Furthermore, let $M_{1}=I-X_{1}\left(X_{1}^{\top} X_{1}\right)^{-1} X_{1}^{\top}$ be the orthogonal projection matrix of the partial model $y=X_{1} \beta_{1}+u_{1}$ so that $M_{1} y=u_{1}$.

Then the OLS estimates of $\beta_{2}$ and of the residuals from equation (1.9) and from

$$
M_{1} y=M_{1} X_{2} \beta_{2}+\text { residuals }
$$

are numerically identical.

Proof 1. See, e.g., Davidson and MacKinnon (2004, p. $68 \mathrm{f}$ ).

The transformed variables $M_{1} y$ and $M_{1} X_{2}$ in (1.10) are the residuals of the partial regressions of $y$ onto $X_{1}$ and of $X_{2}$ onto $X_{1}$, respectively. Hence, they represent transformed versions of $y$ and $X_{2}$, both of them "cleaned" from the partial influence of $X_{1}$. Consequently, the theorem states that the partial impact of $X_{2}$ on $y$ can be estimated in two steps by regressing the residuals from each of the partial regressions onto each other.

In the given context of the VECM, the principle of the Frisch-Waugh-Lovell Theorem turns out to be extremely useful because model (1.8) can be transformed so that the partial impact of the short-run dynamics, quantified by $\Gamma_{i}$, can be concentrated out, i.e., it disappears from the model. The resulting model is "cleaned" from any autoregressive dynamics along time and only consists of "pure" error-correction behavior towards the cointegration relationships. In line with Juselius (2008, p. 149), the transformed version ( $\mathbf{R}$-form) of the full model (1.8) $\left(\mathbf{X}\right.$-form $\left.{ }^{13}\right)$ is obtained by

\footnotetext{
12 Figure 1.3 plots such a specification since $\Gamma_{i}=0$.

${ }^{13} \mathbf{R}$ means reduced and $\mathbf{X}$ denotes the untransformed matrix of right-hand-side variables.
} 
first writing (1.8) in a more compact way as

$$
\begin{aligned}
\begin{array}{c}
Z_{0 t} \\
(v \times 1)
\end{array} & =\Delta p_{t} \\
Z_{1 t} & =p_{t-1} \\
(v \times 1) & =\left(\Gamma_{1}, \Gamma_{2}, \ldots, \Gamma_{k-1}\right) \\
\underset{(v \times(k-1) v)}{\Gamma} & =\left(\Delta p_{t-1}^{\prime}, \Delta p_{t-2}^{\prime}, \ldots, \Delta p_{t-k+1}^{\prime}\right)^{\prime} \\
\underset{(v(k-1) \times 1)}{Z_{2 t}} &
\end{aligned}
$$

so that it becomes

$$
Z_{0 t}=\alpha \beta^{\prime} Z_{1 t}+\Gamma Z_{2 t}+\epsilon_{t}
$$

where $Z_{0 t}, Z_{1 t}, \Gamma=\left(\Gamma_{1}, \Gamma_{2}, \ldots, \Gamma_{k-1}\right)$ and $Z_{2 t}$ are matrices of dimensions $(v \times 1)$, $(v \times 1),(v \times v(k-1))$ and $(v(k-1) \times 1)$, respectively. Based on theorem 1 , the following two auxiliary regressions can be set up:

$$
\begin{aligned}
& Z_{0 t}=\hat{B}_{0}^{\prime} Z_{2 t}+R_{0 t} \\
& Z_{1 t}=\hat{B}_{1}^{\prime} Z_{2 t}+R_{1 t} .
\end{aligned}
$$

The matrices $\hat{B}_{0}^{\prime}$ and $\hat{B}_{1}^{\prime}$ are OLS estimates. Hence the residuals $R_{l t}, l=\{0,1\}$ that would be obtained by regressing $\Delta p_{t}$ and $p_{t-1}$, respectively, on $\Delta p_{t-1}, \Delta p_{t-2}, \ldots, \Delta p_{t-k+1}$ can be calculated as

$$
\begin{aligned}
R_{0 t} & =Z_{0 t}-\hat{B}_{0}^{\prime} Z_{2 t} \\
& =Z_{0 t}-M_{02} M_{22}^{-1} Z_{2 t} \\
& =Z_{0 t}-\left(\frac{1}{T} \sum_{i=1}^{T} Z_{0 t} Z_{2 t}^{\prime}\right)\left(\frac{1}{T} \sum_{i=1}^{T} Z_{2 t} Z_{2 t}^{\prime}\right)^{-1} Z_{2 t} \\
R_{1 t} & =Z_{0 t}-\hat{B}_{1}^{\prime} Z_{2 t} \\
& =Z_{1 t}-M_{12} M_{22}^{-1} Z_{2 t} \\
& =Z_{1 t}-\left(\frac{1}{T} \sum_{i=1}^{T} Z_{1 t} Z_{2 t}^{\prime}\right)\left(\frac{1}{T} \sum_{i=1}^{T} Z_{2 t} Z_{2 t}^{\prime}\right)^{-1} Z_{2 t} .
\end{aligned}
$$

The resulting concentrated model, i.e., the $\mathbf{R}$-form of a VECM then becomes

$$
R_{0 t}=\alpha \beta^{\prime} R_{1 t}+\text { residuals }
$$

so that (1.7) becomes

$$
\begin{aligned}
\text { current 'purged' price movement } & =\text { 'purged' error }- \text { correction } \\
& =g(\text { 'purged' past equilibrium errors }) .
\end{aligned}
$$


The variables of the model are transformed according to Theorem 1 so that the $\Gamma$ parameters disappear. That is, the remaining variables are "purged" from the impacts of the autoregressive dynamics which mix in the $\mathbf{X}$-form of the VECM with the equilibrium adjustment so that $\Delta p_{t}$ is the result of both influences. These influences are on the one hand the pulling forces towards the cointegration relationships and, on the other hand, the inertia forces which link the current price movement to a stronger or weaker extent to past movements. In the $\mathbf{R}$-form in contrast, the left-hand side variables $R_{0 t}$ are only determined by the "purged" deviations from equilibrium $\beta^{\prime} R_{1 t}$ and their adjustment towards the long-run equilibrium. Hence, the $\mathbf{R}$-form corresponds to a VECM without any short-run dynamics $\Gamma_{i}$, i.e., $k-1=0$. Theorem 1 proves that the parameter estimates $\hat{\alpha}$ and $\hat{\beta}$ are identical to the ones of the $\mathbf{X}$-form in (1.8). The regression (1.15) is for cointegrated variables for which $0<\operatorname{rank}(\Pi)=r<v$, also called a reduced rank regression (Anderson, 1951). It is not only the basis for Johansen's (1988; 1991) likelihood-ratio testing and maximum likelihood estimation procedures but also for a number of model specification tests, e.g., as outlined in Juselius (2008, ch. 9).

Figure 1.4 presents three graphs which provide a schematic summary of key characteristics of the model. Hence, we call them characteristic graphs. They will be plotted for each of the models discussed in this thesis for the sake of comparison. The left panel presents a realization of the stochastic mechanism generating the regimes of model parameters, which are in some model classes used to capture the nonlinearities as explained below. It shows the number of the model's regimes and a schmematic depiction of the switching between them. In the case of the VECM, the parameters are assumed to be constant which means that the model possesses one regime. The middle panel plots an example of the adjustment speed $\alpha$ in dependence of the magnitude of the (possibly lagged) deviation from equilibrium quantified by ect. It schematically shows the values of $\alpha$ depending on the regimes, i.e., their assumed behavior in each regime and across the regimes. The right panel depicts the price response $\Delta p_{t}$ in dependence of the magnitude of equilibrium errors of the previous period ect $t_{t-1}$, which results from the regimes of the adjustment behavior in the middle panel. That is, it depicts the error-correction as a function of the past equilibrium errors without regarding short-run dynamics (see equation (1.8), p. 8). For the VECM, there is one possible response which is constant for any magnitude of ect depicted by the bold line of constant slope.

\subsubsection{Estimation}

The estimation of the VECM is not straightforward for cointegrated variables. While the estimates of the short-run dynamics $\Gamma_{i}$ can be obtained via OLS (Lütkepohl and Krätzig, 2004, p. 97) given values of the other two parameter matrices, the estimation of the cointegrating matrix $\beta$ poses a particular challenge for which a number of approaches have been developed. Gonzalo (1994) compares the following estimators 

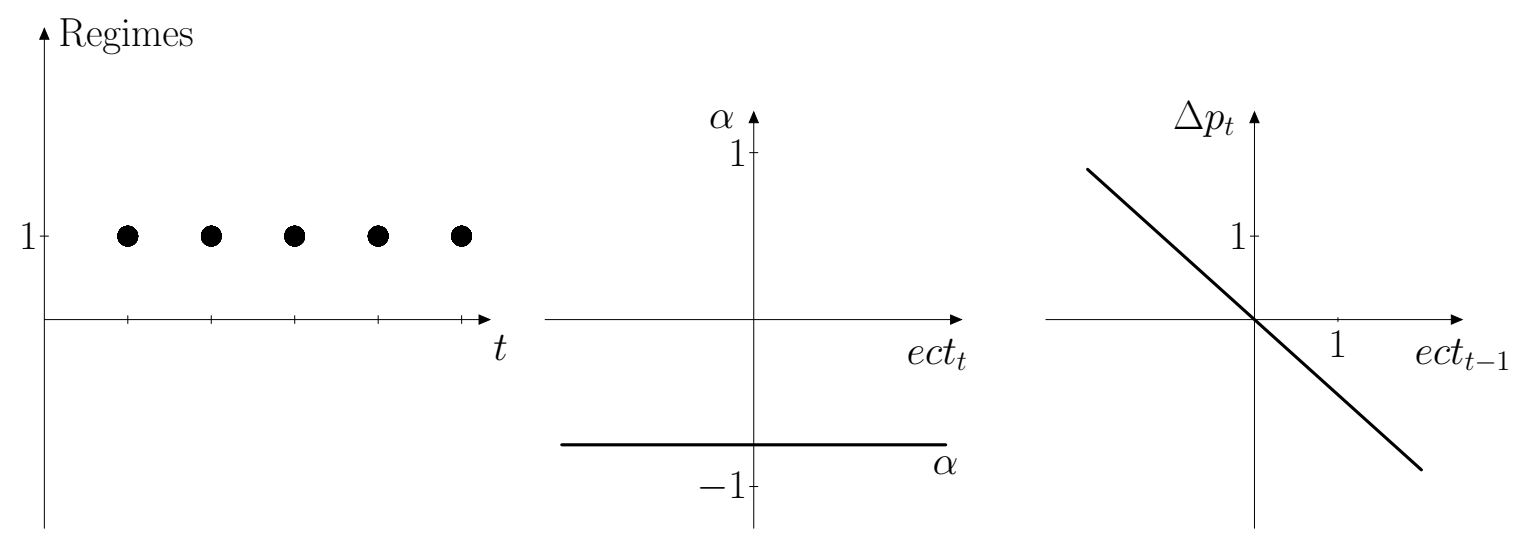

Figure 1.4: Characteristic Graphs of the VECM

regarding their asymptotic distributions and finite sample properties:

1. Engle-Granger two-step OLS regression (Engle and Granger, 1987),

2. nonlinear least squares (Stock, 1987),

3. principal components (Stock and Watson, 1988),

4. canonical correlations (Bossaerts, 1988), and

5. Johansen's (1988) maximum likelihood estimation.

Besides these, methods such as instrumental variables (Hansen and Phillips, 1990), spectral regression (Phillips, 1991), a two-step (2S2) estimator (Lütkepohl and Krätzig, 2004, p. 103 f) and others have been suggested. Recent developments extend estimation to Bayesian analysis, see, e.g., Strachan and Inder (2004). Gonzalo (1994) finds that among the five estimators studied, Johansen's maximum likelihood approach is best in the sense that it is the only method which takes into account all prior knowledge of unit roots, the multivariate character and the dynamics of the system. The resulting estimates have desirable econometric properties and inference can be conducted via usual $\chi^{2}$-tests. The method outperforms the other four in finite samples, i.e., with 100 and 300 observations per time series, respectively, and is found to be robust to nonnormality.

For the Johansen approach, however, lag length selection plays a crucial role since the estimates depend on the chosen number of lags. Simulation results of Gonzalo (1994, p. $220 \mathrm{f}$ ) demonstrate that the method appears to be robust to overparametrization which is the inclusion of a higher order of lags than in the true model. However, if fewer lags than actually needed are included, the approach is outperformed by the method of Engle and Granger (1987). We do not go into more detail for lag length selection but refer instead to detailed accounts, e.g., in Johansen (1995, ch. 2.3.1), Lütkepohl (2007, ch. 4) or Lütkepohl and Krätzig (2004, sec. 2.5.1 and 3.4.1). 
In the following, we briefly outline the steps of the Johansen approach because of its desirable properties. The estimation proceeds stepwise. The formulae are subsequently derived conditionally on parameters assumed to be known. First, the cointegration matrix $\beta$ is estimated via a maximum likelihood approach based on a reduced rank regression, then the loading matrix $\alpha$ can be estimated via OLS. Conditional on the estimated matrix $\Pi$, the short-run parameters $\Gamma_{i}$ can be estimated via equationwise OLS. The core of Johansen's estimation method for the $\alpha$ and $\beta$ parameters is the reduced rank regression of (1.15):

$$
R_{0 t}=\alpha \beta^{\prime} R_{1 t}+\epsilon_{t}, t=1, \ldots, T, \epsilon \sim N_{v}(0, \Omega) .
$$

The assumption of multivariate normality appears to be crucial since it leads to the equality of the maximum of the likelihood function of (1.18) to the determinant of the covariance matrix of the model's residuals as a function of fixed $\beta$ and $\alpha$ :

$$
L_{\max }^{-2 / T}(\beta, \alpha)=|\hat{\Omega}(\beta, \alpha)|+\text { constant terms. }
$$

\section{Step V1: Estimation of the cointegration matrix $\beta$}

$\hat{\alpha}$ can be expressed as a function of $\beta$ solely. Hence, the covariance matrix can be rewritten as a function of only the variables in (1.18) and $\beta$. Its determinant can be factorized into the following terms:

$$
|\hat{\Omega}(\beta)|=\left|S_{00}\right| \cdot \frac{\left|\beta^{\prime}\left(S_{11}-S_{10} S_{00}^{-1} S_{01}\right) \beta\right|}{\left|\beta^{\prime} S_{11} \beta\right|}
$$

where $S_{l m}=T^{-1} \sum_{t=1}^{T} R_{l t} R_{m t}^{\prime}$ and $l, m=\{0,1\}$. The estimates of $\beta$ are then determined as

$$
\hat{\beta}=\underset{\beta}{\operatorname{argmin}}|\hat{\Omega}(\beta)|
$$

resulting from the solution of the eigenvalue problem

$$
\left|\rho S_{11}-S_{11}+S_{10} S_{00}^{-1} S_{01}\right|=\left|\lambda S_{11}-S_{10} S_{00}^{-1} S_{01}\right|=0
$$

where the ratio in (1.20) is "stripped off" the $\beta$ 's. The resulting enumerator is subtracted from the resulting denominator and $S_{11}$ is factored out so that $\lambda=1-\rho$. Hence, the solution $\hat{\beta}$ is the matrix of eigenvectors $w_{1}, \ldots, w_{v}$ ordered according to decreasing eigenvalues $\lambda_{1} \geq \ldots \geq \lambda_{v} \geq 0$. The determinant of the covariance matrix can thus be expressed as

$$
|\hat{\Omega}(\hat{\beta})|=\left|S_{00}\right| \prod_{j=1}^{v}\left(1-\hat{\lambda}_{j}\right) .
$$

The resulting eigenvectors $w$ are not identified. Some normalization has to be carried out for interpretation ${ }^{14}$, for a detailed account on identification, see Johansen (1995,

\footnotetext{
14 The normalization is usually performed by dividing the eigenvector by of one of its elements.
} 
ch. 5) or Juselius (2008, ch. 12-15). The normalized eigenvectors are usually denoted as $\beta_{j}, j=1, \ldots, v$. The larger the estimated eigenvalue $\hat{\lambda}_{j}$, the more pronounced the stationarity of the linear combination $\beta_{j}^{\prime} p_{t}$. Hence the estimated eigenvalues may be used to discriminate between stationary and nonstationary linear combinations. This is the central idea of the Johansen trace test for the number of cointegration relationships, i.e., for the rank $r=\operatorname{rank}(\Pi)$, among the $v$ variables; see, e.g., Johansen (1995, ch. 6) or Juselius (2008, ch. 8).

Step V2: Estimation of the loading matrix $\alpha$

Equation (1.18) can be transformed into

$$
\begin{aligned}
R_{0 t} R_{1 t}^{\prime} \beta & =\alpha \beta^{\prime} R_{1 t} R_{1 t}^{\prime} \beta \\
S_{01} \beta & =\alpha \beta^{\prime} S_{11} \beta
\end{aligned}
$$

so that the OLS estimator of $\alpha$, where $\beta$ is assumed to be given, is

$$
\hat{\alpha}(\beta)=S_{01} \beta\left(\beta^{\prime} S_{11} \beta\right)^{-1} .
$$

Step V3: Estimation of the short-run dynamics $\Gamma_{i}$

The short-run dynamics $\Gamma_{i}$ can be estimated by equationwise OLS assumed that $\Pi=$ $\alpha \beta^{\prime}$ is known (Lütkepohl and Krätzig, 2004, ch. 3.3.2). Based on the notation in (1.11) one obtains:

$$
\begin{aligned}
\underset{(v \times T)}{Z_{0}} & =\left(Z_{01}, \ldots, Z_{0 T}\right) \\
\underset{(v \times T)}{Z_{1}} & =\left(Z_{11}, \ldots, Z_{1 T}\right) \\
\underset{(v(k-1) \times T)}{Z_{2}} & =\left(Z_{21}, \ldots, Z_{2 T}\right) \\
\underset{(k \times T)}{E} & =\left(\epsilon_{1}, \ldots, \epsilon_{T}\right)
\end{aligned}
$$

so that (1.12) can be compactly written as

$$
Z_{0}=\Pi Z_{1}+\Gamma Z_{2}+E
$$

The equationwise OLS estimator is then obtained via

$$
\begin{aligned}
E E^{\prime} & =\left[\left(Z_{0}-\Pi Z_{1}\right)-\Gamma Z_{2}\right]\left[\left(Z_{0}-\Pi Z_{1}\right)-\Gamma Z_{2}\right]^{\prime} \\
& =\left(Z_{0}-\Pi Z_{1}\right)\left(Z_{0}-\Pi Z_{1}\right)^{\prime}-2\left(Z_{0}-\Pi Z_{1}\right) Z_{2}^{\prime} \Gamma^{\prime}+\Gamma Z_{2} Z_{2}^{\prime} \Gamma^{\prime}
\end{aligned}
$$


and

$$
\begin{aligned}
\frac{\partial E E^{\prime}}{\partial \Gamma} & =-2\left(Z_{0}-\Pi Z_{1}\right) Z_{2}^{\prime}+2 \hat{\Gamma} Z_{2} Z_{2}^{\prime} \stackrel{!}{=} 0 \\
\left(Z_{0}-\Pi Z_{1}\right) Z_{2}^{\prime} & =\hat{\Gamma} Z_{2} Z_{2}^{\prime}
\end{aligned}
$$

so that the final estimator of $\Gamma$ becomes

$$
\hat{\Gamma}=\left(Z_{0}-\Pi Z_{1}\right) Z_{2}^{\prime}\left(Z_{2} Z_{2}^{\prime}\right)^{-1} .
$$

In practice, the parameters assumed to be known in each step are replaced by their respective estimates from the step before.

\subsubsection{Interpretation}

Although the method is well established in many fields of research, the interpretation of the estimated parameters in the context of PT analysis is not consistent throughout the literature. Fackler and Goodwin (2001, p. 976) note that "the terminology is often loosely applied, such that the same words may involve distinctly different concepts in different studies". Hence, we try to suggest a terminology which we believe might contribute to improving the clarity of interpretation. We distinguish between the notions of market integration (MI) and price transmission (PT) on the one hand and, on the other hand, aim to provide a meaningful interpretation of the parameters of the VECM. Based on the above thoughts, the mere existence of cointegration relationships among nonstationary prices already contains important information on their relationships.

Fackler and Goodwin (2001, p. 978) define market integration as "a measure of the expectation of the price transmission ratio". Barrett (2001, p. 20) opts to "distinguish between flow-based notions of integration and price-based notions of efficiency based on economic concepts of equilibrium". According to this definition, trade flows are enough to provide evidence of MI since they demonstrate that the product is tradable. Gonzalez-Rivera and Helfand (2001, p. 576) define integrated markets as "the set of locations that share both the same commodity and the same long run information". Consequently, MI appears to be a dichotomous characteristic. However, on page 577, they mention the "degree of integration between locations which belong to the same market". Hence, MI is understood rather as a continuous measure which contradicts the dichotomous character mentioned before in the paper. Thus, for Gonzalez-Rivera and Helfand (2001), MI appears to be both a dichotomous variable as well as a continuous variable.

Our understanding of MI comes closest to that of Gonzalez-Rivera and Helfand (2001) since it encompasses two dimensions of the trading process: the flow of information and a trade-flow-based measure in Barrett's sense. We, however, clearly distinguish between MI and PT. MI is thought of as a long-run characteristic and thus an ex- 
clusively dichotomous variable. PT, in contrast, is seen as having a long-run as well as a short-run dimension, which corresponds to the differentiation between both time horizons in the VECM.

We argue that the mere tradability condition does not suffice to ensure that markets are integrated. For example, the setting in which national policy makers implement prohibitive border protection measures to disconnect the domestic from the international market. Domestic products are nevertheless exported to the world market by a subsidized state trading agency can hardly be viewed as an integrated market although it would be regarded as an integrated market based on the definition of Barrett (2001).

We define MI in the following way: a set of $v$ markets is called integrated if they are all connected by either direct or indirect trade flows and if they are driven by one and only one common factor implying the existence of $r=v-1$ cointegration relationships in the system of prices, each of which consisting of a pair of prices. In this sense, MI appears to be a dichotomous measure, that is, trade flows and $v-1$ long-run relationships are either exhibited in $v$ markets or not.

While MI is thus understood as an exclusive long-run concept, PT covers both long-run and short-run dynamics. PT in the long run is quantified by the slope parameters of the cointegration relationships. That is, we regard it as measured by the coefficients of the prices of the cointegration relationship in the $j^{\text {th }}$ column of the cointegration matrix $\beta .^{15}$ Hence, we understand long-run $\mathrm{PT}$ as a gradual measure since the respective coefficients can take continuous values. The closer they are to zero, the weaker the $\mathrm{PT}$ is in the long run. In the special case in which these slope parameters can be restricted to one, the long-run PT is said to be complete or perfect. ${ }^{16}$ This implies that price shocks are fully transmitted between markets in the long run. The short-run dimension of PT is quantified by the signs and magnitudes of the adjustment speeds. The coefficients in the $j^{\text {th }}$ row of the loading matrix $\alpha$ quantify the magnitudes with which each of the $v$ prices reacts on the $j^{\text {th }}$ disequilibrium relationship from period to period, i.e. the speeds at which a price shock is corrected by the prices. The sign of the respective parameter signals the direction of the adjustment, and its absolute magnitude usually lies between 0 and 1 and thus corresponds to the percentage of any shock which is corrected by the prices in each period. ${ }^{17}$ Thus, PT in the short run is a gradual measure. We regard this distinction as necessary since PT can be complete in the long run albeit slow in the short run. Each of these characteristics describes one aspect of interrelationships of markets in space (see, e.g., Appendix A, pp. 101).

\footnotetext{
${ }^{15}$ If logged data is used in the analysis, these slope parameters are often interpreted as long-run PT elasticities. Different opinions exist in the literature regarding this interpretation; see Lütkepohl (1994), Balcombe and Morrison (2002), Barrett and Li (2002) or Rapsomanikis et al. (2003), who are critical of it, in contrast to Johansen (1995), who shows that this interpretation is valid under some conditions.

${ }^{16}$ Which coincides with the satisfaction of the so-called law of one price, see, e.g., (Fackler and Goodwin, 2001, p. 977) for details.

${ }^{17}$ For a condition which indicates the stability of the estimated model, see footnote 7, p. 6 .
} 
Further insights into spatial interrelationships of prices can be gained from the examination of causality and measures which estimate the time path of the reaction of prices. We do not go into detail here, but refer instead to the literature. Causality can be assessed via concepts such as Granger causality or instantaneous causality (see, e.g., Lütkepohl and Krätzig, 2004, ch. 3.7 and references therein). Measures which estimate the time path of price responses to a shock are:

- Impulse response functions (see, e.g., Lütkepohl and Krätzig, 2004, ch. 4 and references therein),

- Generalized impulse response functions (see, e.g., Koop et al., 1996, and for applications, e.g., Klasra, 2009, or Ubilava and Holt, 2009),

- Nonlinear impulse response functions (see, e.g., Potter, 1995), and

- Persistence profiles (see, e.g., Lee and Pesaran, 1993, and for applications, e.g., Garratt et al., 2006, Ben-Kaabia and Gil, 2007, or Appendix A, pp. 101).

Rapsomanikis et al. (2003) mention another methodological approach besides cointegration, error-correction, and price paths, which they regard as a potentially insightful means to evaluate another aspect of markets' interrelationships in space. This approach is the asymmetric error-correction model (AECM). We do not go into more detail at this stage, as it will be discussed in section 2.1. Here, we point out that such the AECM is one possibility of modeling nonlinearities. Hence, their remark might be reformulated in that it is important for the analyst to identify the source of nonlinearities potentially existing in the data and choose the model which provides an adequate description.

\subsubsection{Applications}

The VECM has been widely applied, for example, in the areas of macroeconomic analysis, financial analysis, analysis of purchasing power parity and the law of one price, and analysis of PT and MI in agricultural or energy markets. Therefore, we do not elaborate on potential areas for applications of this model, but refer instead to the literature. Examples of applications in PT analysis can, e.g., be found in Fackler and Goodwin (2001) or Rapsomanikis et al. (2003). For rich accounts of examples of applications in other areas of economics see, e.g., Enders (2004), Lütkepohl and Krätzig (2004) or Juselius (2008) and the references therein. To our knowledge, only a limited number of publications in agricultural economics exist which analyze a multi-variate setting with more than two variables and more than one cointegration relationship. Some of the few examples are Gonzalez-Rivera and Helfand (2001) or Appendix A, pp. 101. 


\subsection{Nonlinearities in Price Transmission}

In this section we state the focus of this dissertation and confine it from related topics which are beyond its scope.

\subsubsection{The VECM and Nonlinearities}

The fundamental assumption of the VECM in (1.8) is the stability of the parameters of the underlying DGP. That is, the elements of the cointegration matrix $\beta$, of the loading matrix $\alpha$, and of the short-run parameter matrices $\Gamma_{i}$ are assumed to be constant in this model. This restriction implies, together with the traditional specification of the VECM, the three assumptions as outlined in Escribano (2004, p. 77):

1. An unique long-run equilibrium,

2. Error-correction ${ }^{18}$ which takes place as a constant proportion of the previous equilibrium error, and

3. Symmetric adjustment towards equilibrium.

We understand under nonlinearities the relaxation of the crucial assumption of parameter stability. Thus, nonlinearities can have various aspects. Either the long-run equilibrium is no longer unique, or the elements of the loading matrix $\alpha$ are no longer constant. Moreover, the short-run dynamics $\Gamma_{i}$ might also not be constant. Based on the discussion in subsection 1.3.4, pp. 16, nonlinearities can hence appear in the context of PT analysis in the concepts as depicted in Table 1.1.

\begin{tabular}{l|lll}
\hline Aspect & Time horizon & Type of measure & Source \\
\hline Market integration & long-run & dichotomous & Nonconstant cointegration \\
Price transmission & long-run & gradual & Cointegration matrix $\beta$ \\
Price transmission & short-run & gradual & Loading matrix $\alpha$ \\
Short-run dynamics & short-run & gradual & Autoregressive parameters $\Gamma_{i}$ \\
\hline
\end{tabular}

Table 1.1: Aspects of Nonlinearities

Nonlinearities either can originate from one of the mentioned sources or from a combination of several of them. Clearly, the second case is much more complicated. The main challenge from an applied point of view is the identification of the source(s) of nonlinearities in the data which will addressed occasionally in this thesis. A detailed account of identification and testing strategies can be found, e.g., in Juselius (2008, ch. 9). Balcombe and Rapsomanikis (2008) suggest a Bayesian model selection approach. A number of tests for nonlinearities and for model selection have been developed. Nevertheless, considerable research remains to be done in this area.

\footnotetext{
${ }^{18}$ As several other authors, Escribano calls the mechanism governed by $\alpha$ equilibrium-correction.
} However, we stick to the usual terminology in agricultural price analysis, which is error-correction. 
Although essential for applied analysis, we do not deal with identification of and testing for nonlinearities in detail here, but concentrate on the description of various modeling strategies and suitable estimation approaches from an applied point of view. That is, we are most interested in the question of which model class is adequate in which circumstances. Furthermore, what are potentially useful applications of these models to the analysis of empirical questions? Thereby, we restrict the focus of this work to nonlinearities in short-run PT. That is, we relax the assumptions of constant and symmetric adjustment speeds.

The focus of this thesis are nonlinearities in short-run PT which are caused by nonlinear error-correction, that is, by nonconstant $\alpha$ parameters. They are referred to in this dissertation as nonlinearities in price transmission. To our knowledge, this model type was first suggested by Escribano $(1985,1986)$ and Granger and Lee (1989). Although the model is referred to in some literature as nonlinear error-correction (NEC) model (Escribano and Mira, 2002; Escribano, 2004), we call it nonlinear vector errorcorrection model (NVECM) ${ }^{19}$ It generalizes $(1.7)$ in the way that $g(\bullet)$ is allowed to be a nonlinear function of the past equilibrium $\operatorname{errors}^{20}$ :

$$
\text { current price movement }=g(\text { past equilibrium errors })+n(\text { past price movement }) \text {. }
$$

Nonlinear models differ in the specification of the function $g$ which can be modeled in differing ways. Fundamentally, two categories exist. The first consists of parametric functions, often modeled in the form of regime-dependent models. The second alterna-

\footnotetext{
19 The reason for doing so is to avoid confusion. Escribano and Mira (2002) give a general definition of the NEC model which is valid for multivariate models, that is, models of at least two variables and at least one equation (i.e. for at least an ECM). They however, do not explicitly mention whether the model is restricted to one cointegration relationship or applies to the general setting of at least one relationship. Hence, they formulate the model in terms of vectors which justifies calling the model class NVECM. Nevertheless, most theoretical results and all the model's applications so far are, to our knowledge, either obtained for models with one cointegration relationship (Escribano and Mira, 2002) or "single equation NEC models" (Escribano, 2004). Error-correction models (ECM), in contrast to vector ECMs, consist of only a single equation. Hence, we use the term NECM for models consisting of only one equation (and consequently only one cointegration relationship), in accordance with the general terminology of this thesis. The term NVECM, on the other hand, we use for models with more than one equation and at least one cointegration relationship.

20 The NEC model as studied in various publications of Escribano still maintains that $n(\bullet)$ is a linear function. However, several models, some of which will be treated in Chapter 2, also allow $n(\bullet)$ to be nonlinear. That is, along with the adjustment speeds, some models also allow the autoregressive parameters of the short-run dynamics to be nonconstant. However, usually only limited empirical justification or econometric evidence is provided for doing so. The underlying stochastic mechanism generating the nonlinearities in these parameters is in this case usually assumed to be identical to the one of the adjustment speeds (due to the lack of adequate model specification and testing techniques). The setting in which $\alpha$ and the $\Gamma_{i}$ follow different stochastic mechanisms generating nonlinearities seems of course possible from a purely statistical point of view. However, this would yield a highly complex model with problems in the identification of the differing stochastic processes and the estimation and interpretation of the model. Hence, we regard the former case since these the focus of these models lies always on nonlinear error-correction but do not regard the latter case.
} 
tive uses nonparametric functions of the adjustment speeds $\alpha$. Simple examples of the first category are quadratic or cubic polynomials of the equilibrium error as, e.g., in Escribano and Mira (2002). Regime-dependent models may either be used to approximate the true nonlinear stochastic process, usually via piecewise linearity, or a certain type may be used if additional information on the regimes of the DGP is available. The concept of regime-dependent models for the analysis of cointegrated time series was popularized in the area of PT analysis by the publications of Balke and Fomby (1997) and Goodwin and Piggott (2001). The current literature on nonlinearities in models for cointegrated time series only started to emerge 5-10 years later (reviewed in the following subsection). The usage of nonparametric functions has become somewhat popular just recently. Examples are Escribano (2004) or Gaul (2008). Applications in agricultural economics are Serra et al. (2006b) or Goodwin and Vavra (2009).

Regime-dependent models have been quite popular in applied research in the past ten years or so. They are characterized by a number of discrete regimes between which the time series modeled switch back and forth according to a certain mechanism. ${ }^{21}$ This thesis characterizes a number of processes which are suitable to generate various regimes. The DGP of a regime-dependent model may hence be seen to consist of two levels:

1. The stochastic process which generates the regimes and the switching between them, i.e., the regime-generating process $(\mathrm{RGP})^{22}$, and

2. The stochastic process which generates the observations within each regime.

The transition from one regime to another, i.e., the switching, means that at least a subset of the model parameters takes different but constant values. Hence, an alternative perspective on regime-dependent models appears to be interesting. A single regime switch corresponds to a structural break in the dynamic behavior of the time series analyzed since a structural break is nothing else than the change of some or all of the model parameters to new constant values. If only one structural break occurs, the initial set of parameters does not appear again. However, in a regime-dependent model one structural break is followed by more structural breaks so that the model parameters typically return at least one time to their initial values and also to some or all other sets of model parameters (regimes). That is, a regime-dependent model is characterized by recurring structural breaks leading to alternating regimes (sets of constant model parameters) in contrast to a structural break model which typically does not return to a set of parameters.

\footnotetext{
${ }^{21}$ Consequently, they are also referred to as (regime) switching time series models. The nature of the switching mechanism is prespecified by the researcher. The parameters governing the switching process are often estimated from the data (discussed in detail below).

${ }^{22}$ Some authors, such as Saikkonen (2008), call this model part transition function.
} 
This dissertation seeks to shed some light onto the common characteristics of and the differences between the RGPs of

- The threshold vector error-correction model,

- The Markov-switching vector error-correction model,

- The semiparametric vector error-correction model, and

- The parity bounds model,

which will be elaborated in more detail in Chapter 2. We address shortcomings of these models and suggest improvements. As mentioned above, this dissertation pursues an applied perspective. It contributes to the literature by reviewing central properties of several model classes. It discusses potential applications of those in PT analysis and, sporadically, in general applied research in agricultural economics. For selected models, a critique of the methodology is provided and improvements are suggested. Additionally, we present a novel estimation method for a multivariate semiparametric VECM which allows nonparametric modeling of $g$ while the influence of the past price movements $n$ is retained to be a linear parametric function.

\subsubsection{Literature Review}

In this subsection, we briefly review the literature on nonlinear models for cointegrated time series. ${ }^{23}$ We believe that this is useful since most of the econometric literature is comparatively recent. Moreover, we regard it to be a sensible endeavor to give an overview of the recent methodological developments, since most of them are not cited in most of the literature of agricultural PT analysis.

As mentioned above, the first NECMs were developed by Escribano $(1985,1986)$. The AECM was developed by Granger and Lee (1989). Theoretical advances have been developed in Escribano and Pfann (1998), Escribano and Mira (2002) and Escribano (2004). These articles also give a detailed account of the development of the literature during the 1990s. Escribano and Pfann give some formal treatment of nonlinearities in error-correction. They discuss several types of asymmetries in error-correction and give graphical illustrations of these. They suggest rational polynomials as a means to meet necessary stability conditions and illustrate the AECM, i.e., a single equation model, with an example of dynamic labor demand. Escribano and Mira give a thorough treatment of the underlying econometric theory and a representation theorem for NVECMs with one linear cointegration relationship. Moreover, they extend the model to depend on two lags of the equilibrium error and thus provide the link to the smooth regression model of Granger and Teräsvirta (1993).

${ }^{23}$ The literature of particular model classes is discussed later on in the respective sections of Chapter 2, pp. 25, and in Appendix D, pp. 161. 
Escribano (2004) gives a definition of nonlinear cointegration and develops a NECM representation theorem which consists of one cointegration relationship and at least two variables. He suggests a parametric and a semiparametric estimation method where the latter is based on smoothing splines. A short section on tests for nonlinear error-correction based on misspecification tests and on a model selection procedure is provided, in which the semiparametric approach guides the selection of the appropriate parametric nonlinear function. Furthermore, NECMs based on rational polynomials are applied with various estimation techniques to British money demand. The specifications used allow for an interval in which (almost) no error-correction takes place, which is interpreted as manifold equilibria or a "continuum of equilibria," 24 also relaxing the assumption of the traditional VECM of a unique long-run equilibrium. Gaul (2008) develops an innovative approach for the estimation of the threshold VECM and for the estimation of a partially linear VECM. To our knowledge, it represents the first developed estimation method of a NVECM which takes into account the multivariate structure of price systems.

While the approximation of nonlinearities in the loading matrix via regime-dependent models is widely applied in PT analysis and other fields, only a few recent publications use nonparametric approaches to model nonlinearities in the adjustment speeds $\alpha$. Baghli (2005) analyzes the exchange rate of the French Franc/ Deutsche Mark using several types of NECMs such as a cubic polynomial and nonparametric smoothing splines. He estimates nonparametrically, via the Nadaraya-Watson estimator, the partial relationship between the equilibrium error and the change in the dependent variable which is the exchange rate parity. Furthermore, he estimates a threshold ECM and computes several descriptive statistics of generalized impulse response functions. In PT analysis, Mancuso et al. (2003), Serra et al. (2006b), and Goodwin and Vavra (2009), among others, assess the partial relationship between equilibrium errors and price changes using the Nadaraya-Watson estimator to the analysis of EU pork and US meat markets, respectively.

We briefly mention recent publications on nonlinearities other than in adjustment speeds, and further connected topics. Nonlinearities in cointegration relationship(s) are discussed in Gonzalo and Pitarakis (2006) or Karlsen et al. (2007). Bec and Rahbek (2004), Saikkonen (2005) and Saikkonen (2008) investigate the stability of the NVECM. Karlsen et al. (2007) elaborate on testing for the appropriate parametric function. On p. 289, they characterize the current state of research in nonlinearities in models for cointegrated time series as follows: "Nonlinear extensions have centered on both nonlinear error-correction and nonlinear cointegration. It remains to explore possible connections between these models." Along with the issues of model selection and testing, this is an important task for future research in this area.

\footnotetext{
${ }^{24}$ This characteristic comes close to the threshold VECM to be discussed in section 2.1.
} 



\section{Models in Detail}

In this chapter, we aim at giving detailed accounts of the particular forms of the underlying RGPs of selected models suitable for analyzing nonlinearities in short-run $\mathrm{PT}$, that is, nonlinearities in the loading parameters of a VECM.

\subsection{Threshold Vector Error-Correction Model}

This section outlines the most important aspects of regime-dependent time series models whose RGP is determined by the magnitude of a known variable. The threshold vector error-correction model (TVECM) for cointegrated data and the threshold vector autoregressive model (TVARM) for stationary data belong to this model class. This section draws heavily on parts of Appendix D, pp. 161. We therefore provide a rather brief summary here and add some thoughts. The TVARM is not considered here because it represents a well established method which can be found in most advanced textbooks on time series analysis.

\subsubsection{Basic Idea}

The intuition behind the class of threshold time series models is that time series might show a qualitatively different dynamic behavior depending on the magnitude of a certain variable which is called the threshold variable. The qualitative difference is formalized by sets of different parameter values. An important property of this class is that the threshold variable has to be known and quantified. Whenever the threshold variable crosses a certain constant, which is called the threshold, the regime switches. This has the consequence that some or all parameters of the model change in comparison to the former regime. The model parameters are constant within each regime, but are allowed to be different across the regimes. Appendix D, pp. 167, provides a detailed account of the economic rationale behind this model class, which we do not repeat here. Instead, we elaborate an alternative view on this class which turns out to be useful for relating it to other nonlinear time series model classes. We believe that this view facilitates the extension and adaption of the model class to a number of contexts.

In most applications in PT analysis, the following typical specification of the TVECM is used. It analyzes two price series sharing one long-run equilibrium, and regards two thresholds. Since the focus usually lies on nonlinearities in PT (see Table 1.1, p. 
19), the equilibrium error lagged by one period is most often chosen as the threshold variable. This specification is plausible from an economic point of view and consistent with economic theory of trade in space as discussed in Appendix D. Whenever the equilibrium error is larger than a certain threshold value and smaller than a second one (marking the so-called band of no arbitrage), adjustment of disequilibrium, i.e., error-correction, is allowed to be different from outside of this band. Hence, the thresholds which represent the basis of the typical specification can be thought of as constants which discriminate between regimes. Each regime is associated with a set of constant parameter values.

However, this traditional view on the model class may be misleading because it hides the fundamental principle of the model class. The term "threshold" is too restrictive for thinking about extensions and relationships to other model classes. In contrast to the typical specification, the threshold needs neither to be a constant nor to take a single value on the real line. The threshold can rather be thought of as a decision rule which creates mutually exclusive subsets of the set of possible values of the threshold variable, that is, it splits the sample into various regimes. ${ }^{1}$ Each subset represents one regime which is associated with one set of constant parameter values. The model class is hence piecewise linear. The dynamic behavior of the (system of) time series is different across the regimes but constant within each regime. ${ }^{2}$ For the development of the following thoughts, we call the variable inducing the regime switches index in order to distinguish it from the term "threshold" which is in the typical specification reserved for a constant value on the real line. ${ }^{3}$

A decision rule is a general concept. It can consist of one or more constant real numbers partitioning the domain of the index, but can also take much more complex forms. Hence, the principle of the RGP of threshold time series models can be formulated in general as:

$$
\text { index } \in \text { subset } j \Rightarrow \text { set of parameter values } j \Rightarrow \text { regime } j \forall t
$$

where $j$ is one of the possible subsets according to the decision rule. Theoretically, the power set, as the set of all possible sets of the possible values of the index, represents the largest collection of subsets for a given domain. However, not each element of the power set is meaningful in the context of the TVECM as a nonlinear time series model. The empty set is not meaningful because it means that the respective regime effectively

\footnotetext{
1 Therefore, the model class is also called sample splitting or segmented regression (Seo and Linton, 2007).

2 The middle panels of Figures 2.3 and 2.4, pp. 33, illustrate this characteristic.

3 We choose the term "index" following the literature on non- and semiparametric models where the index is a variable which summarizes the effects of several variables (see, e.g., Härdle et al., 2004, ch. 6). This terminology is also used in Seo and Linton (2007) who "allow the threshold variable to be a linear combination of the regressors and/or other variables." (p. 705) The consideration of more than one known variable causing the regime switches represents itself a generalization of the typical specification of the TVECM, and will be addressed below.
} 
does not exist. The set of all possible values is also not meaningful in this context. It would mean that there is only one regime regardless of which value the index takes. Hence, this would denote the VECM which is linear because it possesses only one set of parameter values, i.e., the parameters are constant for the whole sample. The VECM, described in Section 1.3, pp. 7, can thus be seen as a special case of the TVECM. Decision rules are only meaningful for the TVECM if they partition the domain of the index into mutually exclusive subsets. Otherwise, one observation would be classified into more than one regime, that is, the respective observations would simultaneously be generated by more than one parameter set.

The decision rule can be known or unknown. In the latter case, it can either be set exogenously, i.e., imposed on the data, by the researcher or has to be inferred, i.e., estimated, from the data. At this point, the relationship of the threshold model class to dichotomous and dummy variables (sample splitting) becomes apparent. For dichotomous variables, the set of possible values is $\{0,1\}$. Hence, only one decision rule is possible which is known and only two meaningful subsets, $\{0\}$ and $\{1\}$, exist. The decision rule might be formulated in this case as: whenever the index takes the value 0 , one set of parameter values applies; and if it takes 1, the other set. The term "regimes" is rarely used in this setting; however, the elementary principles are identical. Consequently, the dichotomous variable case differs from the typical TVECM described above in two respects. First, the index is not the magnitude of the deviation from equilibrium lagged by one period, that is, a continuous variable, but a dichotomous variable instead. Second, since the dichotomous index can only take two possible values there is only one possibility of a meaningful decision rule. Hence, the decision rule is known and does neither have to be set exogenously nor estimated from the data.

Models using dummy variables are not necessarily identical to the ones based on an intrinsically dichotomous variable since the dummy is created by the exogenous setting of a decision rule. ${ }^{4}$ In this case, the decision rule is determined by the researcher by defining a particular rule to create the dummy variable, possibly derived from theory, etc. Ihle et al. (2010) provide an example of such a special type of a TVECM in the context of PT. They use a dummy variable as index which discretizes the continuous variable measuring trade flows between two locations. That is, the dummy variable takes the value one at time $t$ if trade flows occur and zero otherwise. In PT analysis, an extensive literature of a model class exists which studies asymmetric PT. The socalled asymmetric VECM (AVECM) is characterized by a popular and economically very relevant decision rule. ${ }^{5}$ It was introduced by von Cramon-Taubadel and Fahlbusch (1994), von Cramon-Taubadel and Loy (1996), and von Cramon-Taubadel (1998) (see also Table 2.1, p. 30). In this case, the decision rule consists of partitioning the

\footnotetext{
4 This exogenously determined rule yields the domain of a continuous index to be discretized into two mutually exclusive subsets of values, i.e., a dummy.

${ }^{5}$ Compare p. 18 where the AECM is mentioned. The difference between both models is that the AVECM consists of more than one equation in contrast to the AECM.
} 
deviations from the long-run equilibrium into positive and negative equilibrium errors. That is, the domain of the continuous equilibrium error is discretized according to the

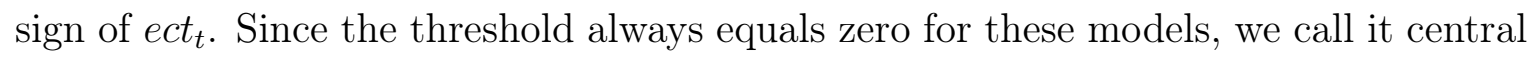
AVECM in contrast to the noncentral AVECM. The latter model also only possesses one threshold which is, however, not restricted to equal zero. Hence, the class of threshold time series models in general and the typical specification of the TVECM in particular encompass these models as special cases as shown in Table 2.1.

\subsubsection{Model Structure}

In this and the following sections, we deal, if not otherwise explicitly mentioned, with the TVECM in the typical specification as described above because this is the form of the model mainly used in PT analysis up to now. The model can be formulated as

$$
\Delta p_{t}=\left\{\begin{array}{l}
\nu^{(1)}+\alpha^{(1)} e c t_{t-1}+\sum_{i=1}^{k-1} \Gamma_{i}^{(1)} \Delta p_{t-i}+\epsilon_{t} \text { if } \text { ect }_{t-1}<\theta^{(1)} \\
\nu^{(2)}+\alpha^{(2)} e c t_{t-1}+\sum_{i=1}^{k-1} \Gamma_{i}^{(2)} \Delta p_{t-i}+\epsilon_{t} \text { if } \theta^{(1)} \leq e c t_{t-1} \leq \theta^{(2)} \\
\nu^{(3)}+\alpha^{(3)} e c t_{t-1}+\sum_{i=1}^{k-1} \Gamma_{i}^{(3)} \Delta p_{t-i}+\epsilon_{t} \text { if } \theta^{(2)}<\text { ect }_{t-1}
\end{array}\right.
$$

where $\theta^{(1)}<\theta^{(2)}$, and $\Delta p_{t}$ is a vector of two prices; the other variables and parameter matrices have suitable dimensions as outlined in Sections 1.2 and 1.3. In applied research, the equilibrium error ect is usually assumed to be lagged by one period, but this may also be adapted depending on the context.

The regime switching mechanism of the RGP of the model class can be endogenous as well as exogenous. In its typical specification (2.2), the equilibrium error, which is a function of the price series regarded, is used as the threshold variable. As dealt with in detail in Appendix D, p. 161, the switching is in this case endogenous because the regime determination according to the magnitude of the equilibrium errors directly depends on the time series studied. The threshold variable $e c t_{t}=\beta^{\prime} p_{t}$ is a linear combination of the prices $p_{t}$ analyzed. If, however, an index is used which is not a function of the prices modeled on the left-hand side of (2.2) - for example, a function of the price of another commodity or the world market price of crude oil instead - the switching mechanism is exogenous.

In Table 2.1, a number of special cases are displayed which derive from the typical specification (2.2) under certain parameter restrictions. Among them are models with two thresholds (TVECM versions), one thresholds (AVECM versions) and no thresholds (VECM). It gives an indication of the flexibility of the model class and its relationships to other nonlinear models in PT analysis. Several of these models are illustrated in this dissertation. For further details, Table 2.1 refers to suitable literature.

Figure D.3, p. 173, displays a realization of a symmetric adjustment equilibrium TVECM (EQ-TVECM) without autoregressive short-run dynamics together with the threshold variable, the thresholds, and the resulting history of regimes. Its upper panel 
shows the evolution of regimes of the model in time. The number of regime switches and the tendency of staying in one regime is mainly determined by properties of the index, which can itself be regarded as a time series (middle panel of the figure). If the index behaves in a nonstationary fashion, a very low number of regime switches in a model like (2.2) can be expected. In other words, a low number of structural breaks in the TVECM parameters is observed. The reason is that the index in this case shows a strong tendency to drift away from its mean. Consequently, most of the time it will be in the outer regimes and tend to stay there. In the case of a model of two regimes, the index will tend to stay in one of them once it has switched to it. Hence, the regimes will tend to be more stable, since they experience only a few switches. A similar behavior can be expected if the index appears to be stationary but shows several intervals of extreme values, as, for example, in Hassouneh et al. (2010). However, if the index is stationary without extreme values, it stays close to its mean because it shows pronounced mean reversion. Consequently, the RGP in this case is characterized by frequent regime switches and comparatively short regime durations, that is, the model experiences frequent structural breaks which lead to frequently alternating regimes.

Figures 2.1 and 2.2 portray typical realizations of a Band-TVECM and an EQTVECM, respectively. In the upper right panels it can be seen that a BAND-TVECM switches more frequently to the outer regimes 1 and 3 and shows higher persistence there, while the EQ-TVECM tends to stay in the middle regime and to switch back to it almost immediately as also mentioned in Lo and Zivot (2001, p. 536). The reason for these differences lies in the differing adjustment (error-correction) behaviors of both models. While only the equilibrium errors that exceed the thresholds are corrected in the Band-TVECM, their total magnitude is adjusted in the EQ-TVECM. This can also be seen in the lower right panels of both figures where the equilibrium errors of the Band-TVECM are markedly more spread out between between the thresholds in the middle regime. Figures 2.3 and 2.4, p. 33, show the characteristic graphs of the Band-TVECM and the EQ-TVECM, respectively. While no error-correction takes place in the middle regime of the Band-TVECM, it may take place in the case of the EQ-TVECM as illustrated in the right panel of both figures. The error-correction of the EQ-TVECM in the middle regime is likely to be weaker than in the outside regimes as indicated by a less steep slope in Figure 2.4 between the thresholds $\theta^{(1)}$ and $\theta^{(2)}$. The middle panels of both figures illustrate the piecewise linearity of the models. In each regime, the adjustment speed $\alpha$ takes a constant value which may, however, differ across regimes. ${ }^{6}$ Consequently the error-correction as depicted in the right panel is hence also constant in each regime (indicated by the constant slope). As shown in the left panels of both figures, regime switches in subsequent periods may only occur between neighboring regimes, that is, between 1 and 2, or 2 and 3 , respectively, but never directly from regime 1 to 3 or vice versa.

\footnotetext{
6 Since both depicted models are symmetric, $\alpha$ is equal in the two outer regimes. One may, of course,
} also consider non-symmetric models which have in each regime a differnt adjustment speed. 


\begin{tabular}{|c|c|c|}
\hline Model name & Parameter restrictions & For details, see, for example, \\
\hline Band-TVECM & $\nu^{(2)}=\alpha^{(2)}=0$ & Balke and Fomby (1997), Lo and Zivot (2001) \\
\hline Continuous Band-TVECM & $\begin{array}{l}\nu^{(2)}=\alpha^{(2)}=0,-\nu^{(1)} / \alpha^{(1)}=\theta^{(1)} \\
-\nu^{(3)} / \alpha^{(3)}=\theta^{(2)}\end{array}$ & Balke and Fomby (1997), Lo and Zivot (2001) \\
\hline EQ-TVECM & $\nu^{(1)}=\nu^{(2)}=\nu^{(3)}=0^{a}$ & Balke and Fomby (1997), Lo and Zivot (2001) \\
\hline Symmetric TVECM & $\left|\alpha^{(1)}\right|=\left|\alpha^{(3)}\right|,\left|\theta^{(1)}\right|=\left|\theta^{(2)}\right|$ & Balke and Fomby (1997), Lo and Zivot (2001) \\
\hline Symmetric adjustment TVECM & $\left|\alpha^{(1)}\right|=\left|\alpha^{(3)}\right|$ & Balke and Fomby (1997), Lo and Zivot (2001) \\
\hline Symmetric thresholds TVECM & $\left|\theta^{(1)}\right|=\left|\theta^{(2)}\right|$ & Meyer (2004) \\
\hline (Central) AVECM & $\theta^{(1)}=\theta^{(2)}=0, \nu^{(1)}=\nu^{(3)}=0^{b}$ & $\begin{array}{l}\text { Meyer and von Cramon-Taubadel (2004), Frey } \\
\text { and Manera (2007) }\end{array}$ \\
\hline Noncentral AVECM & $\theta^{(1)}=\theta^{(2)}, \nu^{(1)}=\nu^{(3)}=0$ & Subervie (2008), Hassouneh et al. (2010) \\
\hline VECM & $\begin{array}{l}\theta^{(1)}=\theta^{(2)}=0,\left|\alpha^{(1)}\right|=\left|\alpha^{(3)}\right|, \nu^{(1)}= \\
\nu^{(3)}=0, \Gamma_{i}^{(1)}=\Gamma_{i}^{(3)} \forall i\end{array}$ & Juselius (2008) \\
\hline
\end{tabular}

Note: For a more detailed classification of models for the analysis of asymmetric PT, and more examples of decision rules, see, e.g., Frey and Manera (2007, Table 5).

${ }^{a}$ This model may have error-correction in the middle regime $\left(\alpha^{(2)} \neq 0\right.$ as in Figure 2.4) or not $\left(\alpha^{(2)}=0\right)$.

${ }^{b}$ In the case that the two thresholds of $(2.2)$ coincide, the middle regime does not exist, that is, all parameters with index ${ }^{(2)}$ do not exist.

Table 2.1: Special Cases of the TVECM 


\section{Extensions}

The TVECM itself is a special case of the smooth transition VECM (STVECM) developed by Teräsvirta (1994). ${ }^{7}$ The STVECM generalizes the TVECM in such a way that the regimes change smoothly instead of by discrete shifts from one set of parameter values to another. We do not discuss this model class here in detail; applications in PT analysis can be found in Serra et al. (2008) or Ubilava and Holt (2009).
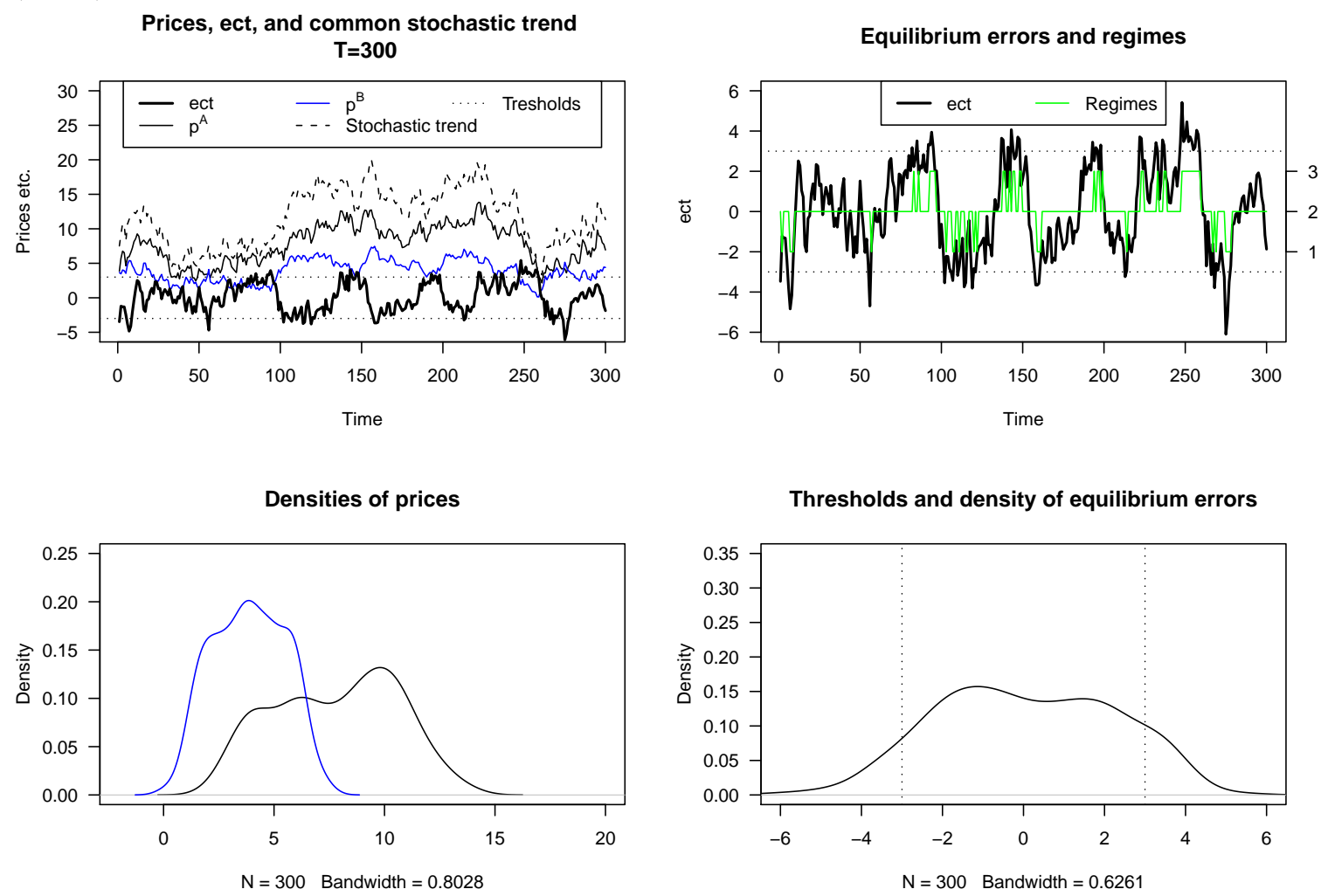

Note: This graph is based on identical innovations $\epsilon_{t}$ and $\eta_{t}$ as the one in Figure 1.3, p. 9. For details, see Balke and

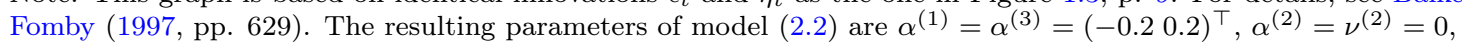
$\beta=(1-2)^{\top},\left|\theta^{(1)}\right|=\theta^{(3)}=3$, and $k=1$, i.e., $\Gamma_{i}^{(1)}=\Gamma_{i}^{(2)}=\Gamma_{i}^{(3)}=0$.

Figure 2.1: Example of a Continuous and Symmetric Band-TVECM

We see the most potential for extensions of the model class in the index and the decision rules creating the regimes. For example, the assumption of constant thresholds, interpreted as constant transaction costs, may be replaced by various modeling strategies of time varying thresholds as, e.g., in Bekkerman et al. (2009). They relax the assumption of constant thresholds by defining the index as a function of fuel prices and seasonal components instead. They model seasonally varying thresholds by using trigonometric functions. The price difference between two markets, that is, a restricted cointegration relationship, is chosen to be the threshold variable. The

\footnotetext{
$\overline{7}$ For an overview see, e.g., van Dijk et al. (2002) and Teräsvirta (2004). For further generalizations see, e.g., Saikkonen (2008). Kristensen and Rahbek (2007) develop likelihood-based inference for this model class.
} 
regimes are determined by the magnitude of this variable relative to the seasonally varying thresholds.

Prices, ect, and common stochastic trend $\mathbf{T}=\mathbf{3 0 0}$

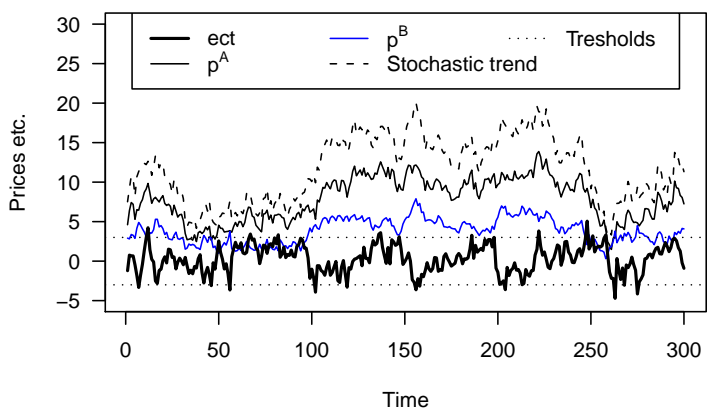

Densities of prices

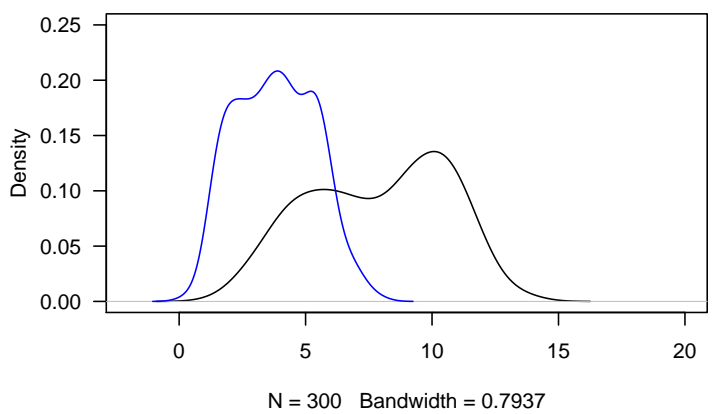

Equilibrium errors and regimes

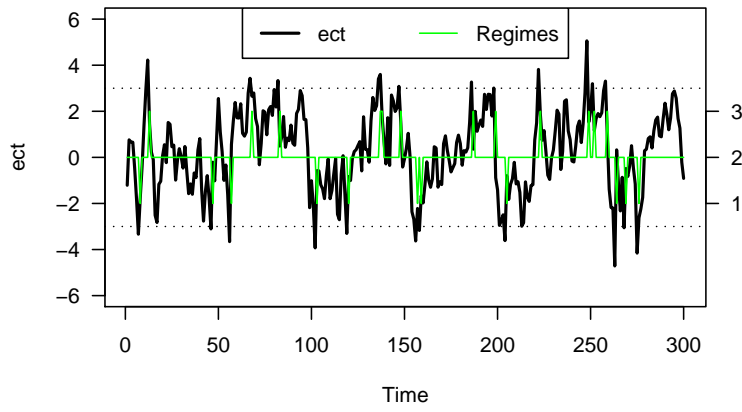

Thresholds and density of equilibrium errors

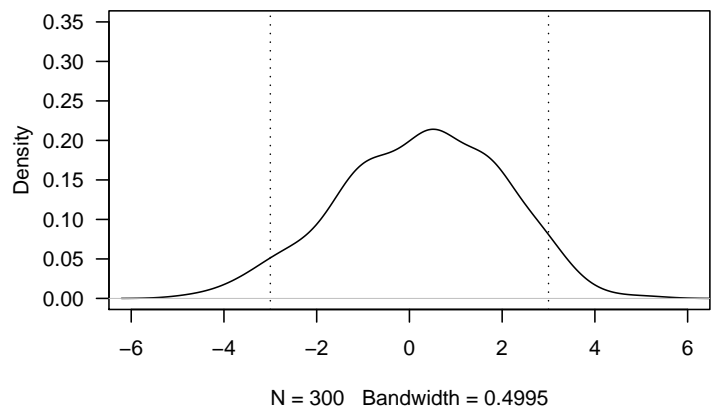

Note: This graph is based on identical innovations $\epsilon_{t}$ and $\eta_{t}$ as the one in Figure 1.3. For details, see Balke and Fomby (1997, pp. 629). The resulting parameters of model $(2.2)$ are $\alpha^{(1)}=\alpha^{(3)}=(-0.20 .2)^{\top}$, $\alpha^{(2)}=\nu^{(1)}=\nu^{(2)}=\nu^{(3)}=0, \beta=(1-2)^{\top},\left|\theta^{(1)}\right|=\theta^{(3)}=3$, and $k=1$, i.e., $\Gamma_{i}^{(1)}=\Gamma_{i}^{(2)}=\Gamma_{i}^{(3)}=0$.

Figure 2.2: Example of a Symmetric EQ-TVECM

However, a range of variables and decision rules seem plausible for being regarded as triggering regime switches in short-run PT. Hassouneh et al. (2010) study the impact of public alertness on regimes of PT. They construct an index based on the intensity of media reporting on BSE which might plausibly evoke regimes in short-run PT. In other words, variables related to the flows of physical goods across space or to information regarding producer or consumer decisions seem to be promising choices of suitable indices. Moreover, variables affecting production or consumption other than information are plausible. For each extension, the connection between the index and the model parameters should be well established based on economic theory since a high number of potential indices may potentially be used. The choice and justification of an appropriate index/ threshold variable and a suitable decision rule remains the task of the researcher.

Furthermore, the index may be generalized to be a function of more than one variable. In a setting in which regimes are simultaneously determined by two or more economic variables, this seems to be a promising option. The variables of the index may be summarized in a linear combination. Alternatively, a two-dimensional decision rule 


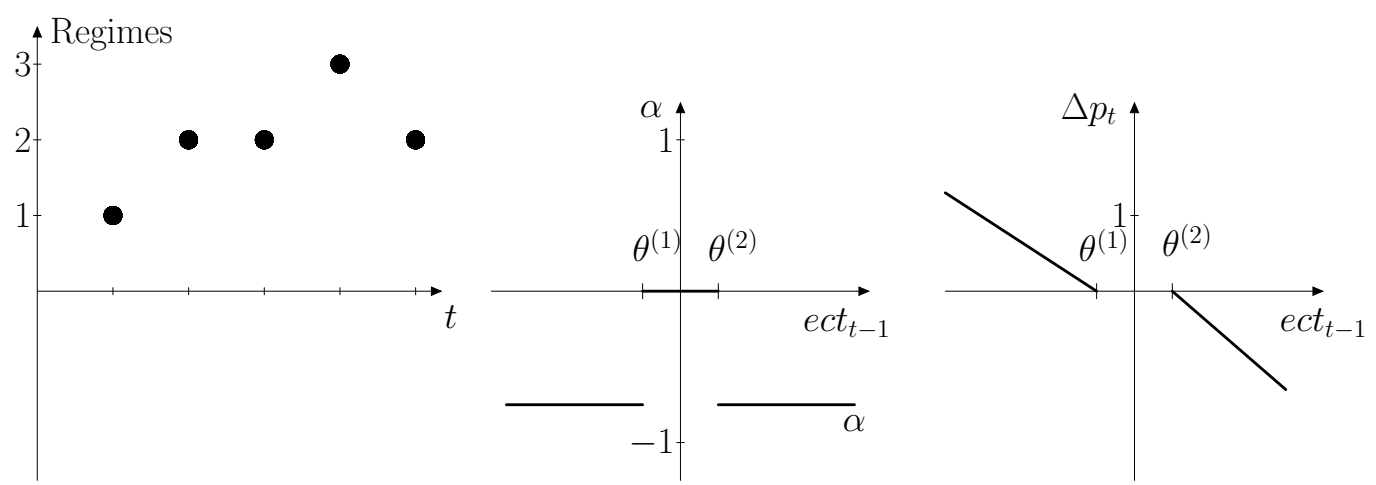

Figure 2.3: Characteristic Graphs of a Typical Sym. and Continuous Band-TVECM might be plausible. Based on this rule, each individual variable of the index falls into subsets of its own domain. One could also imagine that each subset $j$ of the index might not only be associated with a set of parameter values but also with a particular functional relationship of them. That is, the functional form of the model can be thought of as being regime-dependent. However, although these conceptual extensions might be useful from an applied point of view, such analyses appear to be very challenging since the econometric theory for estimation and inference of most cases is not yet developed. ${ }^{8}$
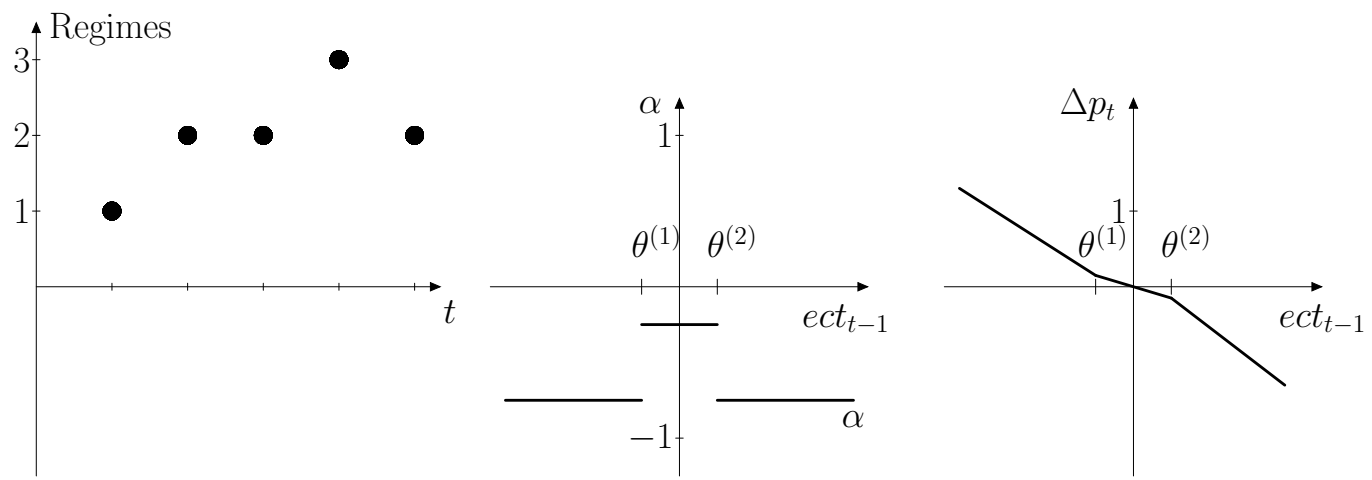

Figure 2.4: Characteristic Graphs of a Typical Symmetric EQ-TVECM

\subsubsection{Estimation}

Various approaches have been suggested for estimation of the TVECM, as discussed in detail in Appendix D, pp. 173, and Table D.1, p. 174. Several more approaches have been developed recently. Seo (2007) and Seo and Linton (2007) develop the estimation procedure and theory of a smoothed least squares estimator for the TVECM with two regimes. The idea of their approach is to substitute the part of the estimation's

\footnotetext{
8 The derivation of theory for simple models is already demanding, as discussed in detail in Seo and Linton (2007); most results for these so far have been obtained under rather strong assumptions. Hence, the analysis of more complex models poses even more challenges.
} 
objective function which indicates the regimes with a nonparametric expression in the form of a kernel. The objective function thus contains a nonparametric component; in other words, it becomes smoothed. Gaul (2008) suggests an estimation method for a TVECM with three regimes and proves the consistency of the threshold estimators. Furthermore, he develops a method for estimating the cointegration rank in each regime and a supLM test for linearity. Kourtellos et al. (2009) develop a concentrated least squares estimator for a two-regime TVECM for a specific setting. They assume an error term in the index which is allowed to be correlated with the errors of the equations in the two regimes.

\subsubsection{Interpretation}

Some or all of the parameters of the model may be allowed to be regime-dependent. Their interpretation in each regime is identical to the linear VECM. The magnitudes of the estimated thresholds, that is, their magnitudes relative to variables of the model or further descriptive variables, may also be of interest. For a constrained version of the typical TVECM, the estimated thresholds have the particular interpretation as transaction costs as outlined in detail in Appendix D1, pp. 175.

Based on the estimation results, a time series or a history of the regimes and regime switches can be constructed as, e.g., in the upper panel of Figure D.3, p. 173. The frequency and timings of switches may provide interesting insights into the market(s) studied. For example, the timings of the regime switches can be compared to known events which occurred during the sample period. Furthermore, an impulse response analysis might yield interesting insights into the market dynamics. A possible measure is nonlinear impulse response functions as a special case of generalized impulse response functions; see, e.g., Bekkerman et al. (2009). Alternatively, persistence profiles can be estimated which might serve for calculating the periods needed to adjust half of any equilibrium shock, the so-called half-lives, as, e.g., in Ben-Kaabia and Gil (2007).

\subsubsection{Applications}

The TVECM can be regarded as an established methodology in PT analysis. Both its typical specification and special cases, as outlined in Table 2.1, are widely applied. Table DI.5, pp. 200 in Appendix DI, gives a comprehensive overview of applications of this model and its versions in PT analysis. 


\subsection{Markov-Switching Vector Error-Correction Model}

This section outlines the most important aspects of regime-dependent time series models whose RGP is governed by a Markov chain. The Markov-switching vector errorcorrection model (MSVECM) for cointegrated data and the Markov-switching vector autoregressive model (MSVARM) for stationary data belong to this model class. This section draws heavily on parts of Appendix D, pp. 161. We therefore provide a rather brief summary here and add only a few thoughts. An application of the MSVARM can be found in Appendix B, pp. 125.

\subsubsection{Basic Idea}

The concept of modeling the RGP of time series as a Markov chain was developed by Hamilton (1989). The VECM version of the model was independently published by several authors, e.g., Jackman (1995), for the analysis of presidential approval, Hall et al. (1997) for the analysis of house prices, and Tillman (2004) for studying interest rates. Krolzig $(1996,1997)$ develop a detailed treatment of MS time series models. The model class is suitable for a particular form of nonlinearities characterized by Hamilton (1989) as "discrete shifts in regime-episodes across which the dynamic behavior of the series is markedly different." Hamilton and Raj (2002b) mention that "normal behavior of economies is occasionally disrupted by dramatic events that seem to produce quite different dynamics for the variables that economists study." Psaradakis et al. (2004a) note that the MSVECM is "best suited to situations where the change in regime is triggered by a sudden shock to the economy, situations which might not be adequately described by models with smooth transitions or threshold effects." Furthermore, van Dijk et al. (2002, p. 28) note that MS models "are most useful when it is impossible to identify a suitable switching or threshold or transition variable or when it is impossible to assume that this variable is continuous."

The intuition behind MS time series models is that the (system of) time series is subject to alternating and recurring regimes which switch from one to another with certain probabilities in subsequent periods. The regime changes (switches) occur in a stochastic manner. Hence, the underlying RGP is assumed to yield a discrete number of (often qualitative) regimes. The model class is particularly adequate to analyze settings characterized by recurring discrete shifts between regimes if the variables triggering them are not known or not measurable.

Hamilton (1994, ch. 22.4) emphasizes the strengths of this model class, which are comprehensiveness and great flexibility. Furthermore, it can be used, in contrast to other model classes, in bivariate as well as in multivariate settings. However, from an empirical research point of view, its merit lies in the property that the factors inducing the regime switches do not have to be specified. This means that the determinants leading to the regime switches do not have to be observable and even may not be known to the researcher a priori. In the light of the problem of data availability that the 
applied researcher is often faced with, such a property appears to be attractive. On the other hand, this characteristic poses the challenge to the researcher to identify plausible determinants which triggered the recurrent switches a posteriori. General driving forces of trade and prices, and a number of further economic variables belong to these determinants. In the context of PT analysis, regime-switching seems plausible, which may not be determined by the magnitude of disequilibrium, but rather by exogenous factors of the surrounding political or economic system, or by the "general state" of the trading process. PT behavior might temporarily change in the sense that two or three economic states alternate due to external factors such as general characteristics of the political or economic system. Raj (2002) mentions national policy changes, economic recessions, financial panics, and wars as potential determinants in the context of business cycle analysis. Further "sources of abrupt change" such as government actions in the form of the introduction or the elimination of legal regulations are alluded to in Hamilton (1995). Chamley (1999) shows that a unique equilibrium may exist in a world characterized by imperfect information showing episodes of high and low economic activity. These episodes may, among other consequences, result in regimes in disequilibrium adjustment and short-run dynamics of PT which are randomly switching.

In this context, the criticism might be put forward that the incidences of objective events such as changes in a country's trade or agricultural policy, further trade-relevant governmental actions, or wars are observable. Hence, the regimes can be deduced from this and do not have to be estimated. Consequently, MS time series models would neither be the most adequate choice for modeling nonlinearities nor would they be necessary. Nevertheless, the usage of the MSVECM or MSVARM to model such contexts may be appropriate. The reason is that the behavior of economic agents, might they be producers, traders, transporters, sellers, consumers, etc., does not necessarily coincide with the incidence of objective events. We argue that it is mainly the economic agents and their collective behavior in response to objective events rather than the events themselves which generate the realizations of economic variables studied by economists, e.g., price series. ${ }^{9}$ An example is anticipated uncertainty about the near future due to elections and the following expected (potentially fundamental) policy changes. Furthermore, behavior of agents might change due to insecurity caused by turmoil in politically unstable countries. Moreover, exceptional positive or negative expectations about the near economic future such as forecasts of strong price rises or pronounced price depressions, crop failures, etc. might be reasons. ${ }^{10}$ Economic agents' behavior changes not only due to the occurrence of objective events but already due to subjective perceptions of and expectations towards objective events. For example, agents' behavior changes not only in consequence of armed conflicts, but already due the fear of armed conflicts alone.

When the changed behavior of individual agents becomes collective action, that is,

9 For a recent account, see, e.g., Akerlof (2009).

${ }^{10}$ For an example, see Agra Europe (2008, middle of page M/3): "Importers still panic buying...". 
enough individuals change their behavior in the same or in a similar way, it becomes manifest or is at least mirrored in observable variables created by this behavior, let it be trade quantities, prices or further socio-economic variables. The class of MS time series models uses such observed variables as a source of econometric information. Therefore, it captures the agents' changing behavior rather than the unobserved or unknown incidents of objective events. We believe that this distinction is important because one aspect may, but does not necessarily, coincide with the other. MS time series models evaluate observed data in order to obtain evidence on regimes and the associated sets of parameter values. Hence, they are apt to capture shifts in collectively perceived subjective expectations and feelings towards more or less fundamental changes of the socio-economic or political framework of a group of economic agents. The model class provides indications about the timings and magnitude of changes in economic agents' behavior in response to such exogenous changes. Obtaining measurements on variables inducing the changes is a challenging task in this context; in most cases it will not be feasible at all. The stochastic approach towards the regime switches is justified by the collective action needed to impact economic variables. Because the collective action is the sum of the behaviour of many individuals it can hardly be regarded as deterministic or predictable.

The agents' subjective perception of an objective event might lead to the phenomenon that their behavior changes a considerable time before an event - maybe an announced elementary policy change - starts, because of anticipated objections, fears, etc. On the other hand, it can lead to inertia of agents' behavior. A certain behavior may continue considerable time after an event has finished or has been implemented due to broken trust, subjective extrapolation of today's difficult situation to tomorrow, pessimistic perceptions of the near future, etc. The latter phenomenon can be observed in the current global financial crisis, which was elicited by the collapse of the US housing bubble in 2007. However, similar mechanisms are plausible to happen on all economic scales. In the economic literature such phenomena are termed nonfundamentals, market psychology, animal spirits, sunspots or self-fulfilling prophecies. ${ }^{11}$ Furthermore, some time lag may lie between the formal announcement of governmental actions and their effective implementation, for example, because the organization of the implementation lasts several weeks or months. In addition, actions implemented on a national or regional level might not be published since the government has no interest in this, so that the exact incidences of obvious events are not even known. Agricultural scandals, as they occasionally occur in Europe and the Unites States, may result in temporary changes of consumer demand which might lead to transitionally different transmission of price signals. Further events such as crop failures, transient demand or supply, or asymmetries of business cycles may temporarily impact trade and price dynamics.

\footnotetext{
${ }^{11}$ For a brief literature review on this topic, see p. 178 in Appendix DI.
} 


\subsubsection{Model Structure}

As mentioned above, the class of MS time series models is suitable for both stationary (MSVARM) and cointegrated variables (MSVECM). We briefly outline the structure of the model class following Krolzig (1997). Its flexibility becomes apparent in its three general formulations:

1. MSVARM of intercept form:

$$
p_{t}=\nu^{\left(S_{t}\right)}+\sum_{i=1}^{k} \Pi_{i}^{\left(S_{t}\right)} p_{t-i}+\epsilon_{t}
$$

2. MSVARM of mean adjusted form:

$$
p_{t}-\mu^{\left(S_{t}\right)}=\sum_{i=1}^{k} \Pi_{i}^{\left(S_{t}\right)}\left(p_{t-i}-\mu^{\left(S_{t-i}\right)}\right)+\epsilon_{t}
$$

\section{MSVECM:}

$$
p_{t}=\nu^{\left(S_{t}\right)}+\alpha^{\left(S_{t}\right)} \beta^{\prime} p_{t-1}+\sum_{i=1}^{k-1} \Gamma_{i}^{\left(S_{t}\right)} p_{t-i}+\epsilon_{t}
$$

where $\epsilon_{t} \sim N I D\left(0, \Sigma^{\left(S_{t}\right)}\right)$.

The terms $\nu^{\left(S_{t}\right)}, \Pi_{i}^{\left(S_{t}\right)}, \Gamma_{i}^{\left(S_{t}\right)}, \alpha^{\left(S_{t}\right)}$ and $\mu^{\left(S_{t}\right)}$ denote dependence of the respective parameters on the regime $S_{t}$, which is a realization of the specific RGP of the model class, namely a Markov chain. The regime variable is allowed to be unobservable. For a certain time $t$, it takes an integer value between 1 and the total number of regimes $M$, i.e., $S_{t}=s \in\{1,2, \ldots, M\}$, where $M$ is seldom larger than three in applications. Hence, each regime-dependent variable is again regarded as piecewise constant, that is, constant in each regime $S_{t}=s$. For example, $\alpha^{\left(S_{t}\right)}=\alpha^{(s)}$ is piecewise constant in the following way:

$$
\alpha^{\left(S_{t}\right)}= \begin{cases}\alpha^{(1)} & \text { if } S_{t}=1 \\ \vdots & \\ \alpha^{(M)} & \text { if } S_{t}=M .\end{cases}
$$

Although all model parameters in specifications (2.3) to (2.5) except $\beta$ might be conditioned on regimes, in most cases in practice it suffices to regard only a subset of them as regime-dependent. In the context of cointegrated variables and nonlinear PT, model (2.5) is appropriate where $\alpha$, and maybe also $\Sigma$, are allowed to be regime-dependent. Often, a switching intercept is not appropriate $(\nu=0)$. The $\Gamma_{i}$ may also be assumed to be regime dependent. However, the focus of modeling almost always lies on the nonlinearities in PT (see Table 1.1, p. 19). 
Krolzig (1997, ch. 13) suggests the following classification of the model class:

- $\mathrm{MS}^{*}(M)-\operatorname{VAR}(k)$ for stationary variables

- $\operatorname{MS}(M)-\operatorname{VECM}(k-1)$ or $\operatorname{MSCI}(M, r)-\operatorname{VAR}(k)$ for cointegrated variables

where $M, k$ and $r$ are defined as above. The placeholder $*$ can be one or a combination of the following specifications:
A for Markov-switching autoregressive parameters,
H for Markov-switching residual covariance matrix,
I for Markov-switching intercept (equations (2.3) and (2.5)), and
M for Markov-switching mean (equation (2.4)).

For example, a MSMH(2)-VAR(1) denotes a model with a Markov-switching mean with two regimes, regime-dependent error covariances, and one autoregressive lag. ${ }^{12}$ Note that a MSM-VAR and a MSI-VAR are in contrast to their linear counterparts, not equivalent. They imply instead quite different behaviors of the estimated constants. While constant, i.e., the mean of the variable, jumps immediately to its new level after a regime switch in the first case, it approaches its new value smoothly in the latter case, as depicted in Figure 2.5.

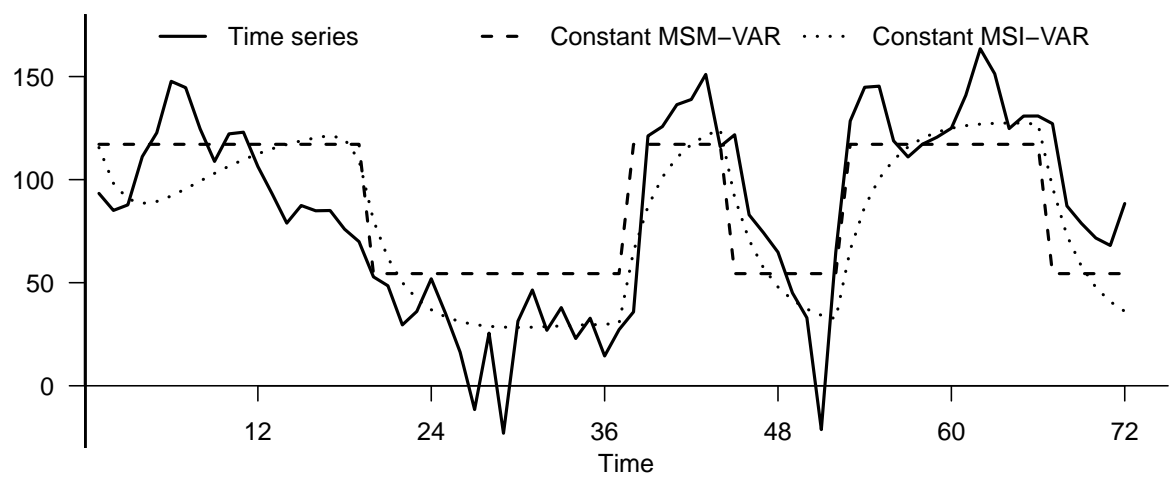

Figure 2.5: Estimated Constants of a MSM-VAR and a MSI-VAR Specification

The core of the RGP of this model class is a hidden Markov chain of $M$ states as, e.g, depicted in Figure D.4, p. 180. This RGP is thus also modeled as piecewise linear, since each state is associated with a set of constant parameter values of the time series model. Hence, the regime-dependent parameters are constant in each regime but are allowed to change across regimes. The properties of the Markov chain determine the regime switching. The chain is assumed to be ergodic, irreducible, and homogeneous. The first characteristic means that a stationary distribution of regimes exists. The second condition ensures that any regime can switch to any other regime, i.e., no

\footnotetext{
${ }^{12}$ In addition, exogenous variables may be considered in the model, whose coefficients may also be allowed to be regime-dependent (Krolzig, 1997, section 10.1). In Krolzig's terminology, such a specification would be called $\mathrm{MS}^{*}(M)-\operatorname{VARX}(k)$.
} 
'absorbing' states are allowed for. This excludes cases in which the chain has two states and only one regime switch occurs, so that it then stays infinitively long in the second regime and never switches back. The third assumption states that the transition probabilities are constant. More details are given in Appendix D, pp. 179.

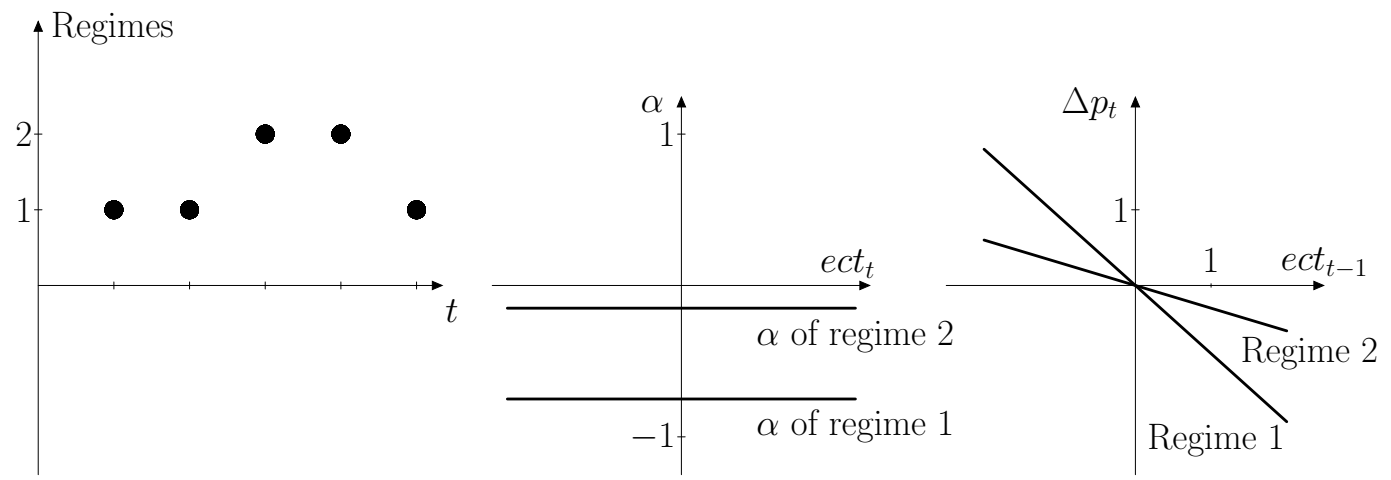

Figure 2.6: Characteristic Graphs of a Typical MSVECM

As discussed above, the regimes of the model class are assumed to be generated by exogenous factors which impact production, trade and consumption. Hence, the RGP is exogenous in the sense that the regimes do not depend on the price series. That is, the adjustment speeds are, in contrast to the typical specification of the TVECM, independent of the equilibrium error (see Figure 2.6). The price series are shaped instead by exogenous regimes evoked by a changing trade policy, recession vs. boom, the seasons of a year, etc. Furthermore, the so-called Markov property ensures that the RGP is memoryless. That is, the switching to a new state at some point in time only depends on the state of the chain in the previous period, not on periods more in the past. Hence, the regime switching is independent of its history. Examples of typical realizations of a MSVECM of two regimes are displayed in Figures 2.7, p. 41, and D.5, p. 181, respectively. Figure D.5 indicates by the shaded areas how the behavior of the time series is exogenously determined by the states of the Markov chain which are displayed in its upper panel.

\section{Extensions}

A number of publications relax the assumption of homogeneity, i.e., transition probabilities are no longer assumed to be constant, but instead allowed to depend on fundamentals of the economy, characteristics of the country, etc. Diebold et al. (1994), Hamilton and Raj (2002a), Schaller and van Norden (2002), Camacho (2005) or Jerzmanowski (2006) are examples. Pelagatti (2005, 2008) proposes a duration-dependent MSVARM in which the transition probabilities depend on how long the Markov chain has been in a particular state. Hall et al. (1997) suggest a MS time series model whose RGP is endogenous in the sense that the transition probabilities depend on the magnitude of disequilibrium. (Krolzig, 1997, ch. 10), Bhar and Hamori (2007), and $\operatorname{Kim}(2004,2009)$ allow for endogenous regime switching by letting the transitions probabilities be a function of the time series analyzed. 

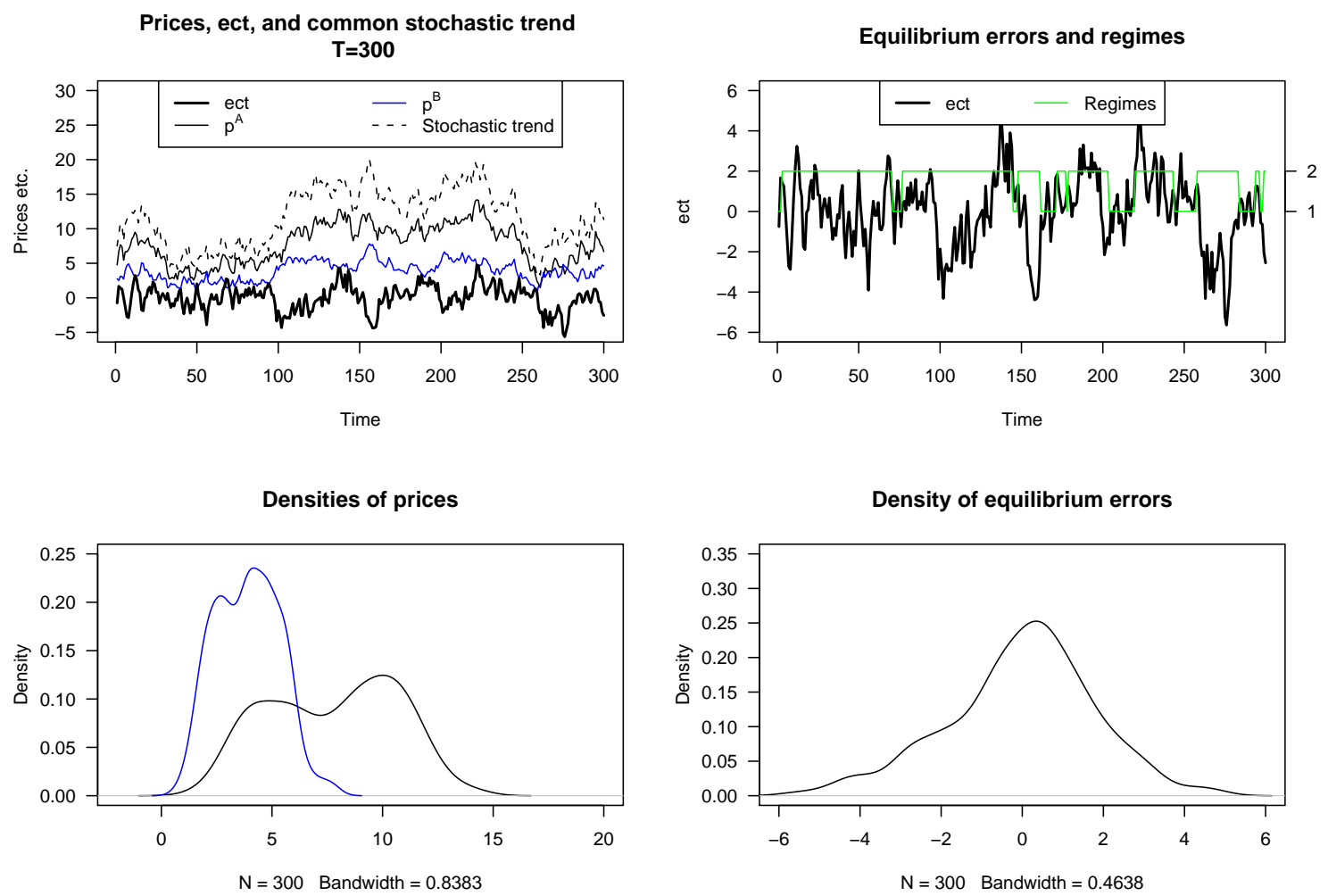

Note: This graph is based on identical innovations $\epsilon_{t}$ and $\eta_{t}$ as the one in Figure 1.3. For details, see Balke and Fomby (1997, pp. 629). The parameters of model (2.5) are $M=2$, transition matrix: $\left(\begin{array}{cc}0.9 & 0.1 \\ 0.05 & 0.95\end{array}\right), \nu^{(1)}=\nu^{(2)}=0$, $\alpha^{(1)}=\left(\begin{array}{lll}-0.2 & 0.2\end{array}\right)^{\top}, \alpha^{(2)}=(-0.030 .03)^{\top}, \beta=(1-2)^{\top}, \Sigma^{(1)}=\Sigma^{(2)}$, and $k=1$, i.e., $\Gamma_{i}^{(1)}=\Gamma_{i}^{(2)}=0$.

Figure 2.7: Example of a MSVECM

\subsubsection{Estimation}

Several methods are available for the estimation of this model class; most popular is the Expectation-Maximization-Algorithm (EMA), of which Krolzig (1997) and Mizrach and Watkins (2000) give detailed accounts. A verbal description of the EMA is provided in Appendix D, pp. 181, and Figure D.6, p. 182. Bayesian techniques to estimate MS time series models are also suggested by some authors (Krolzig, 1997, ch. 8; Frei, 2008). For the MSVECM, a two-step procedure is adopted. First, the equilibrium errors are estimated via, say, the Johansen approach. They are then included in the MSVARM as an exogenous variable; for details, see Krolzig (1996, 1997, ch. 13).

\subsubsection{Interpretation}

MS time series models are suitable for the identification and characterization of discrete regime shifts when quantitative information of the variables causing them is not available to the researcher. They provide estimates of the temporal occurrences and of the magnitudes of regime switches. Besides globally constant and regime-dependent parameters, probability statements on the regime incidences are estimated. These statements consist of information on the RGP, the so-called transition probabilities, and on the regime incidences, called the filtered probabilities and the smoothed probabilities, respectively. 
The transition probabilities are the probabilities $\gamma_{s s^{\prime}}=\operatorname{Pr}\left(S_{t+1}=s^{\prime} \mid S_{t}=s\right)$ for the switching of the Markov chain to state $s^{\prime}$ in the subsequent period conditional on the regime $s$ in the current period, that is, the chances that the parameter set of the (system of) time series will switch to regime $s^{\prime}$, given its current regime $s$. Hence, the chances of staying in the current regime are also estimated. Based on these estimates, the expected duration $\hat{\lambda}^{s}$ of regime $s$ can be obtained via $\hat{\lambda}^{s}=\mathrm{E}\left[\lambda \mid S_{t}=\right.$ $s]=\frac{1}{1-\hat{\gamma}_{s s}}$ (Krolzig, 1997, subsection 11.3.4) where $\hat{\gamma}_{s s}$ denotes the estimated transition probability of staying in regime $s$ (see the transition matrix (D.15), p. 179). The filtered probability of each of the $M$ regimes quantifies the chances that an observation belongs to the respective regime at time $t$ conditional on the data up to this time and the estimated model parameters. The smoothed probabilities give the probabilities for the occurrences of each of the $M$ regimes at time $t$ conditional on the in total available information, i.e., the entire sample at hand (see, for an example, Figure 3 in Appendix B).

Each period can then be assigned to one of the $M$ regimes. Possible criteria are the assignment to the regime which has a smoothed probability of at least 0.5 in this period, which, however, can lead to many unclassified time points in the case of more than two regimes. Alternatively, it can be assigned to the most probable regime, i.e., the regime with the largest smoothed probability. In this way, the regime switches can be timed, and a history of the regimes for the sample at hand can be constructed (see, for an example, Figure 4 in Appendix B). Based on this regime history, several questions can be addressed. For example, regime-specific values of descriptive variables, which were not regarded in the time series modeling, may be calculated. Thus, the researcher can obtain evidence on the particular characteristics of each regime, and on potential determinants which induced the identified regime switches. Hence, conclusions on the extent of the behavioral change of economic agents can be drawn. The coincidences of estimated regimes with known objective events, as discussed above, might also be assessed. Furthermore, tests on the estimated time series parameters can be conducted via a number of testing procedures as, e.g., outlined in Krolzig (1997, ch. 7). Ehrmann et al. (2001) and Tillman (2003) suggest algorithms for estimating impulse response functions for MS time series models.

\subsubsection{Applications}

Applications of the model are mainly found in business cycle and financial research; see for a review, for example, Appendix B, pp. 125, or Appendix D, pp. 177. Recent applications beyond the traditional fields are, for example, de Morais and Portugal (2004), who assess structural changes in the Brazilian import demand. Jerzmanowski (2006) analyzes the relationship between institutions and growth paths. Owyang et al. (2007) analyze factors affecting employment growth regimes across US cities. Cologni and Manera (2009) study the effects of oil shocks on output growth. 
In PT analysis, the model class is rarely applied. Only recently, the MSVECM was used by Brümmer et al. (2009) to analyze vertical PT between wheat and flour in Ukraine. Busse and Brümmer (2009a), Busse and Brümmer (2009b) and Busse and Ihle (2009) use the methodology for analyzing biofuel markets and their linkages to vegetable oil and fuel markets. Appendix B, pp. 125, provides an analysis which uses the MSVARM. The setting which the model is applied to represents a suitable example for the discussion in subsection 2.2.1, p. 35. The approach is chosen to analyze the potential effects of export bans on margins of maize prices between major production and consumption regions in Eastern Africa. If repeatedly introduced export bans were effective, trade flows would have been repeatedly altered. Consequently, average margins should be markedly different in periods with and without export restrictions. However, neither was the knowledge on ban incidences complete, nor was it clear whether the enforcement of the bans was effective and varied over time. Thus, several sources of uncertainty about possible regimes existed.

In the context of agricultural economics, changes in collective behavior of economic agents may either be directly exogenously imposed, e.g., due to governmental decisions, or resulting from responses to economy-wide or societal developments. Moreover, characteristics of agricultural production processes and complex interdependencies in subsectors might be determinants. Examples for the first case are recurring but unknown or unobservable policy regimes, such as temporarily implemented foreign trade restrictions (see example in Appendix B, pp. 125). Examples for the second case are different types of economic cycles in agriculture, such as cyclic development of prices or livestock production, most famously the pork cycle. Investment, production and growth cycles in agriculture or the development of industry-specific work-force are further variables which seem suitable to be analyzed by MS time series models. Such analyses would correspond to business cycle analysis in subsectors of economies in which the RGP is approximated by two or three discrete regimes representing, e.g., expanding and contracting phases. Moreover, phases of structural change in agriculture during the last decades might be looked at. Economic agents' response to recurring phases of conflict or unrest might be an interesting topic to study. Finally, the model class appears suitable for both horizontal as well as vertical PT analysis in assessing price phases or regimes of disequilibrium adjustment. Analyses using this model class might study uni- or multivariate time series which might be stationary or cointegrated. Hence, quantities such as prices in levels or changes, price margins, trade quantities, growth rates, or investment behavior can be assessed. 


\subsection{Semiparametric Vector Error-Correction Model}

This section outlines a flexible model class for analyzing nonlinearities in PT which encompasses the classes of the linear VECM and the nonlinear TVECM as special cases. It models the adjustment of the equilibrium errors flexibly via nonparametric estimation. The autoregressive short-run parameters, however, are regarded as not exhibiting nonlinearities. Thus, their impact is modeled linearly. Since one part of the model is modeled nonparametrically while the rest of the model is kept parametrically, it is called the semiparametric vector error-correction model (SPVECM). ${ }^{13}$

\subsubsection{Basic Idea}

The SPVECM immediately reflects the considerations of Table 1.1, p. 19, regarding nonlinearities in PT which is caused by nonconstant adjustment parameters in $\alpha$. Instead of trying to find an adequate parametric approximation of the nonlinearities, the functional form of the adjustment is estimated from the data.

This model is applicable in a number of contexts since it does not requiere the choice of a specific (parametric) RGP. Regime-dependent models such as the TVECM or the MSVECM approximate nonlinearities by discrete regimes. The STVECM, which is not discussed in this thesis, relaxes the discontinuous shifts between sets of parameter values and allows for smooth transition between them. However, in each of these model classes the researcher has to decide on certain parameters and/ or (parametric) functional forms. That is, subjective choices have to be made at several decisive modeling stages, for example, based on an intimate expert knowledge of the markets to be analyzed or based on previous analysis or on the existing literature. In other cases, the necessary detailed knowledge for model choice might not be available, or even if it is, the subsequent choices might be inadequate.

The SPVECM allows for flexible modeling based on the data at hand without imposing parametric restrictions on the form of nonlinearities as depicted in the characteristic graphs in Figure 2.8. The approach enables the data to speak to the researcher about whether it contains nonlinearities and of which form they are. Based on such explorative information, the researcher might then make an informed guess on a suitable parametric approximation. This fact can be seen by comparing the middle panels of, e.g., Figures 2.8, p. 47, and 2.3 or 2.4, p. 33, respectively. While the EQ-TVECM models a nonconstant adjustment speed $\alpha$ via various regimes in each of which $\alpha$ is constant, the SPVECM models nonlinearities in one regime in which $\alpha$ many take any smooth function. Consequently, the price response appears similar in both graphs. However, it is modeled smoothly by the SPVECM, e.g., in contrast to the TVECM.

\footnotetext{
${ }^{13}$ One could, of course, consider also a semiparametric vector autoregression or corresponding nonparametric models, see, e.g., Tschernig (2004). We, however, focus on the SPVECM in this dissertation.
} 


\subsubsection{Model Structure}

We first repeat the structure of the VECM (1.8), p. 8, in order to contrast it to the SPVECM. The linear VECM can be formulated as

$$
\Delta p_{t}=\operatorname{corr}^{\mathrm{VECM}}+\sum_{i=1}^{k-1} \Gamma_{i} \Delta p_{t-i}+\epsilon_{t}
$$

where corr $^{\mathrm{VECM}}$ is the error-correction in case of the VECM, in particular:

$$
\operatorname{corr}^{\mathrm{VECM}}=\alpha \beta^{\prime} p_{t-1}=\left(\begin{array}{ccc}
\alpha_{11} & \ldots & \alpha_{1 r} \\
\vdots & \ddots & \vdots \\
\alpha_{v 1} & \ldots & \alpha_{v r}
\end{array}\right) \beta^{\prime} p_{t-1}
$$

where $\alpha$ is a matrix of real constants. In case of the SPVECM, the error-correction is no longer linear but consists of smooth functions instead, so that

$$
c o r r^{\mathrm{SPVECM}}=\left(\begin{array}{ccc}
m_{11}\left(\beta^{\prime} p_{t-1}\right) & \ldots & m_{1 r}\left(\beta^{\prime} p_{t-1}\right) \\
\vdots & \ddots & \vdots \\
m_{v 1}\left(\beta^{\prime} p_{t-1}\right) & \ldots & m_{v r}\left(\beta^{\prime} p_{t-1}\right)
\end{array}\right)
$$

Hence, equation (1.29), p. 20, can be rewritten to

$$
\text { current price movement }=m(\text { past equilibrium errors })+n(\text { past price movement })
$$

where $m(\bullet)$ only is assumed to be a smooth function which can take many flexible forms (e.g. as in the middle panel of Figure 2.8). Since no parameters are estimated, the functions $m_{k l}\left(\beta^{\prime} p_{t-1}\right), k=1 \ldots, v ; l=1 \ldots, r$ can only be plotted. Examples can be found in Gaul (2008, Figure 3.1). ${ }^{14}$ The model structure corresponds to the so-called semiparametric partial linear model (PLM) as, e.g., outlined in Härdle et al. (2004, ch. 7).

\subsubsection{Estimation}

The estimation of the SPVECM is a straightforward application of the Speckman estimator or profile likelihood approach for the estimation of PLMs. Gaul (2008, sec. 3.3) gives a detailed account which we sketch briefly. For this purpose we adapt equation (1.12), p. 11, in the following way:

$$
\begin{aligned}
& Z_{0 t}=m\left(\beta^{\prime} Z_{1 t}\right)+\Gamma Z_{2}+\epsilon_{t} \\
& Z_{0 t}=m\left(e c t_{t-1}\right)+\Gamma Z_{2}+\epsilon_{t} .
\end{aligned}
$$

\footnotetext{
$\overline{{ }^{14} \mathrm{He} \text { develops an estimation theory and }}$ applies the model to high-frequency German stock market data. His estimates of the form of error-correction closely resemble a Band-TVECM.
} 


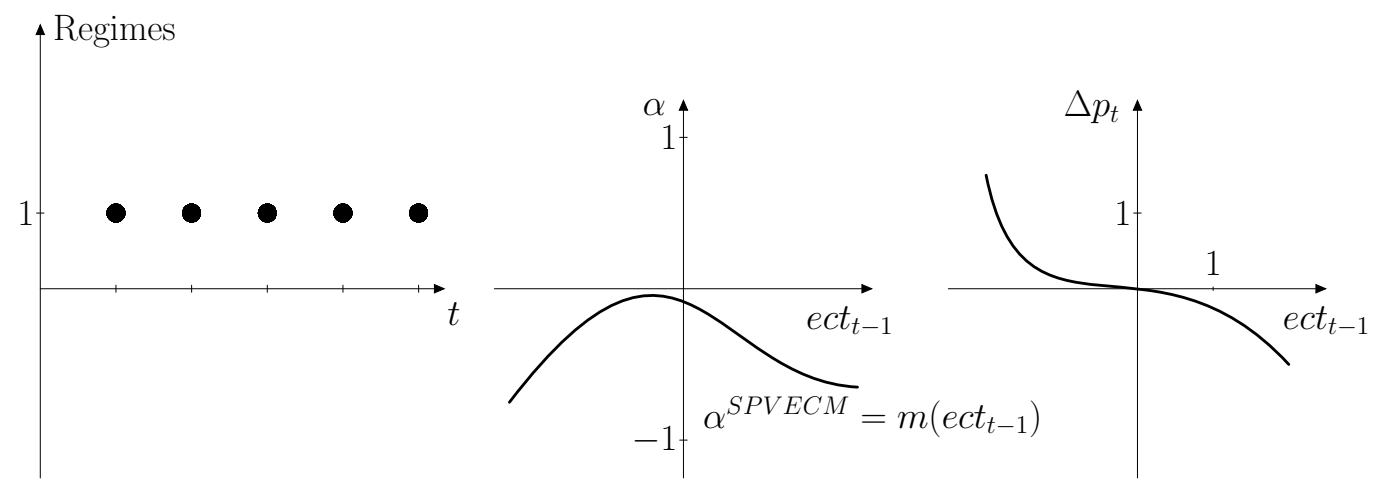

Figure 2.8: Characteristic Graphs of a Typical SPVECM

The estimation proceeds in similar steps as outlined for the VECM in section 1.3.3, pp. 12. The first step, i.e., the estimation of the cointegration matrix $\beta$, is identical. The next two steps are reordered and adapted according to the Speckman estimator for PLMs. Hence, second, the estimate of the parametric part $\Gamma$ is obtained. In the third step, the nonparametric part $m$ is estimated.

Step SP1: Estimation of the cointegration matrix $\beta$

Identical to step $V 1$ of the estimation of the VECM, p. 14.

Step SP2: Estimation of the short-run dynamics $\Gamma_{i}$

The model is cleaned from the potentially nonlinear impact of the equilibrium errors ect $t_{t-1}$. By taking conditional expectations, the following concentrated model ${ }^{15}$ is obtained:

$$
\begin{aligned}
Z_{0 t}-E\left(Z_{0 t} \mid e c t_{t-1}\right) & =\Gamma\left(Z_{2}-E\left(Z_{2} \mid e c t_{t-1}\right)\right)+\epsilon_{t} \\
Z_{0 t}^{*} & =\Gamma Z_{2}^{*}+\epsilon_{t} .
\end{aligned}
$$

$\hat{\Gamma}$ could be estimated via OLS if $Z_{0 t}^{*}=Z_{0 t}-E\left(Z_{0 t} \mid e c t_{t-1}\right)$ and $Z_{2}^{*}=Z_{2}-E\left(Z_{2} \mid e c t_{t-1}\right)$ were known. Gaul suggests to estimate the conditional means via nonparametric regression based on the Nadaraya-Watson estimator in order to obtain a feasible estimator of $\Gamma$ :

$$
\begin{aligned}
\hat{w}\left(Z_{0 t} \mid e c t_{t-1}\right) & =\frac{1}{T h} \sum_{j=1}^{T} \frac{Z_{0 j}}{\hat{f}\left(e c t_{t-1}\right)} K\left(\frac{e c t_{t-1}-e c t_{j-1}}{h}\right) \\
\hat{w}\left(Z_{2} \mid e c t_{t-1}\right) & =\frac{1}{T h} \sum_{j=1}^{T} \frac{Z_{2}}{\hat{f}\left(e c t_{t-1}\right)} K\left(\frac{e c t_{t-1}-e c t_{j-1}}{h}\right)
\end{aligned}
$$

\footnotetext{
${ }^{15}$ Note that this is the same principle which leads to the $\mathbf{R}$-form of a VECM, p. 11. Hence, this model version might be called the $\mathbf{R}^{\mathbf{S P}}$-form.
} 
where $w(\bullet)$ is a smooth function. The quantity $\hat{f}\left(e t_{t-1}\right)$ denotes the kernel estimator of the density of the equilibrium errors $f\left(e^{e c t} t_{t-1}\right)$, where $K(\bullet)$ is a kernel function and $h$ is the so-called bandwidth which decides on the degree of smoothness:

$$
\hat{f}\left(e c t_{t-1}\right)=\frac{1}{T h} \sum_{j=1}^{T} K\left(\frac{e c t_{t-1}-e c t_{j-1}}{h}\right) .
$$

In order to avoid numerical problems, $(2.12)$ is multiplied by $f\left(e t_{t-1}\right)$ which yields the density weighted estimates:

$$
f\left(e c t_{t-1}\right) Z_{0 t}^{*}=\Gamma f\left(e c t_{t-1}\right) Z_{2}^{*}+f\left(e c t_{t-1}\right) \epsilon_{t} .
$$

In order to obtain the feasible OLS estimator of the short-run dynamics $\Gamma$, the quantities in (2.15) are replaced by their estimates from (2.13) and (2.14) so that

$$
\begin{aligned}
\hat{\Gamma}= & \frac{\sum_{t=1}^{T}\left(Z_{0 t}-\hat{w}\left(Z_{0 t} \mid e c t_{t-1}\right)\right)\left(Z_{2}-\hat{w}\left(Z_{2} \mid e c t_{t-1}\right)\right)^{\prime}\left(\hat{f}\left(\widehat{e c t}_{t-1}\right)\right)^{2}}{\sum_{t=1}^{T}\left(Z_{2}-\hat{w}\left(Z_{2} \mid e c t_{t-1}\right)\right)\left(Z_{2}-\hat{w}\left(Z_{2} \mid e c t_{t-1}\right)\right)^{\prime}\left(\hat{f}\left(\widehat{e c t}_{t-1}\right)\right)^{2}} \\
= & \frac{\sum_{t=1}^{T} \hat{Z}_{0 t}^{*} \hat{Z}_{2}^{* \prime}\left(\hat{f}\left(\widehat{e c t}_{t-1}\right)\right)^{2}}{\sum_{t=1}^{T} \hat{Z}_{2}^{*} \hat{Z}_{2}^{* \prime}\left(\hat{f}\left(\widehat{e c t}_{t-1}\right)\right)^{2}}
\end{aligned}
$$

where $\widehat{e c t}_{t-1}=\hat{\beta}^{\prime} Z_{1 t}=\hat{\beta}^{\prime} p_{t-1}$ are the estimated equilibrium errors and $\hat{\beta}$ is estimated in step $S P 1$.

Step SP3: Estimation of the nonparametric adjustments $m_{k l}\left(\right.$ ect $\left._{t-1}\right)$ in $\alpha^{S P V E C M}$

The result $\hat{\Gamma}$ of step $S P \mathcal{2}$ can be substituted for $\Gamma$ into (2.11) so that it becomes:

$$
\begin{aligned}
Z_{0 t} & =m\left(e^{e c t_{t-1}}\right)+\hat{\Gamma} Z_{2}+u_{t} \\
Z_{0 t}-\hat{\Gamma} Z_{2} & =m\left(e c t_{t-1}\right)+u_{t} \\
\tilde{Z}_{0 t} & =m\left(e t_{t-1}\right)+u_{t}
\end{aligned}
$$

This is again a nonparametric regression which can be estimated using the NadarayaWatson estimator in the following way:

$$
\hat{m}(z)=\frac{\sum_{t=1}^{T} \tilde{Z}_{0 t} K\left(\frac{z-e c t_{t-1}}{h}\right)}{\sum_{t=1}^{T} K\left(\frac{z-e c t_{t-1}}{h}\right)} .
$$


Thus, a parametric estimate of the linear influences of the short-run dynamics $\Gamma$ and nonparametric estimates of $m(\bullet)$ are obtained.

\subsubsection{Interpretation}

For the SPVECM, interpretation of the parameters is the same as for the VECM. However, the aspects particular to this model are the plots of the estimated functions $\hat{m}(\bullet)$. Pointwise confidence intervals may be estimated as in Gaul (2008) who also develops a nonparametric test for linearity. For model selection, which in this context is the choice of the optimal bandwidth $h$, he uses four different criteria: cross validation, Shibata's Model Selector, Akaike's Information Criterion, and the Final Prediction Error.

\subsubsection{Applications}

To our knowledge, the SPVECM has not yet been applied except in Gaul (2008) and Gaul and Theissen (2008). However, several studies in PT analysis have used similar nonparametric approaches. Mancuso et al. (2003) analyze interest rates using a local linear regression estimator without regarding short-run dynamics alternatively to a threshold autoregressive model. Serra et al. (2006b) follow a similar methodological approach to analyze European pork markets. Goodwin and Vavra (2009) apply a Nadaraya-Watson estimator to a VECM which they assume not to have any short-run dynamics. ${ }^{16}$

We see one useful application of this approach in exploratory analysis in order to identify the functional form of the nonlinearities prevalent in the data at hand. It may guide the researcher in the choice of a suitable RGP for a given data set and can, hence, provide valuable information on price dynamics. Furthermore, it might shed light on the question of whether the $m_{k l}\left(e t_{t-1}\right)$ are similar or whether possibly differing functional forms appear. The approach allows for more than one cointegration relationship. It thus can be seen as a multivariate extension of, e.g., the TVECM not only in the sense that it flexibly estimates the functional form of the adjustment but it also is capable of revealing the adjustment in multivariate analyses.

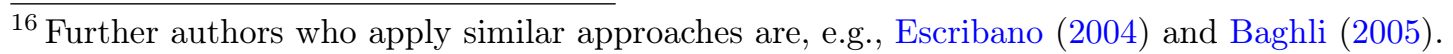




\subsection{Parity Bounds Model}

This section outlines the most important aspects of the regime switching model class for PT analysis which is usually known as the parity bounds model (PBM). The model is described and contrasted to the aforementioned models based on cointegration analysis. Although dealing with time series data, the model class remains silent on how the regimes evolve in time, i.e., on the nature of the RGP, but rather focuses on the results of this mechanism and classifies the data by a flexible mixture distribution approach into various regimes.

\subsubsection{Basic Idea}

The model class was first proposed by Spiller and Huang (1986) consisting of two regimes. The publication was a reaction to the article of Stigler and Sherwin (1985) of the title The Extent of the Market, in which they proposed a simple parallel price movement test. Sexton et al. (1991) extended the Spiller-Wood-approach to three regimes. The methodology was then revived by Baulch $(1994,1997)$ extending the model to the PBM. Most often, versions of Baulch's specification with some modifications are used, which we therefore focus on in the following.

The basic idea of this model class consists of the classification of a measure quantifying the size of costs for spatial arbitrage, that is, the transaction costs, in relation to the price differences between two markets into several regimes. Transaction costs are the sum of a number of components associated with conducting trade, such as costs for transport, insurance, financing, duties, or risks premia. ${ }^{17}$ Negassa and Myers (2007) suggest the following definition of transaction $\operatorname{costs} \tau_{t}^{A B}$ between markets $A$ and $B$ :

$$
\tau_{t}^{A B}=\delta+X_{t} \beta+e_{t}
$$

where $X_{t}$ contains the components of these costs, $\beta$ the respective weights, and $\delta$ is a constant. Both, $\beta$ and $\delta$ are to be estimated and may differ across market pairs. The difference of the price spread and the transaction costs between two markets, i.e., the rents to spatial arbitrage, hence becomes:

$$
R_{t}^{A B} \equiv p_{t}^{B}-p_{t}^{A}-\tau_{t}^{A B}
$$

Positive rents are the motivation for traders to engage in spatial arbitrage. The higher they are, the more additional profit the arbitrageur will earn from the performed transaction. They are thought of as a random variable that varies around zero. It corresponds to the equilibrium errors of the time series models outlined above, since the parity of price differences and transactions costs corresponds to long-run equilibrium of the aforementioned models. The difference lies in the fact that transaction costs are

\footnotetext{
${ }^{17}$ For a detailed account of components of transaction costs, see, e.g., Barrett (2001). In case that the model is applied to vertical price analysis, the processing costs and the price differences between the processing stages have to be regarded.
} 
always estimated in time series models, while they are partly based on (some of their) observed components in the context of the PBM. However, recent publications using the PBM show a tendency to account more precisely for these costs. Padilla-Bernal et al. (2003) and Moser et al. (2009) predict total transaction costs with a number of explanatory variables, which are then used in the estimation of the model.

Transaction costs are the core element of the model class since they define when parity prevails and when not. At the same time, they also make up the quantity which is afflicted with the most uncertainty, since it is a highly complex conglomerate of a large number of potential components on which reliable data is hardly available. Based on economic theory and the available data, three or, alternatively, six regimes are derived in which the rents may fall according to the state of the market. A certain distribution is assumed for each regime which are, with associated weights, combined into the likelihood function of the sample for estimation. The uncertainty in regime classification is accounted for by calculating the so-called parity bounds, that is, the $90 \%$ or $95 \%$ confidence interval of the parity regime around the (estimated) transaction costs.

\subsubsection{Model Structure}

Basically, three regimes are considered by the model, which are defined in terms of the size of the rents to spatial arbitrage $\left(R_{t}^{A B}=0, R_{t}^{A B}>0, R_{t}^{A B}<0\right)$, that is, the size of the price differential between two markets relative to the transaction costs. The rents to spatial arbitrage are decomposed into one or two error terms, depending on the regime, in the following way:

$$
R_{t}^{A B}=\left\{\begin{array}{cl}
\nu_{t}^{A B} & \text { if } R_{t}^{A B}=0 \text { (Regime 1) } \\
\nu_{t}^{A B}+u_{t}^{A B} & \text { if } R_{t}^{A B}>0 \text { (Regime 2) } \\
\nu_{t}^{A B}-e_{t}^{A B} & \text { if } R_{t}^{A B}<0 \text { (Regime 3) }
\end{array}\right.
$$

For each of the three error terms, a certain distribution is assumed as

$$
\begin{array}{ll}
\nu_{t}^{A B} \sim & N I D\left(0, \sigma_{\nu}^{2}\right) \\
u_{t}^{A B} \stackrel{i i d}{\sim} & N^{+}\left(0, \sigma_{u}^{2}\right) \\
e_{t}^{A B} \stackrel{i i d}{\sim} & N^{+}\left(0, \sigma_{e}^{2}\right)
\end{array}
$$

where $N^{+}(\bullet)$ denotes the half-normal distribution. Hence, the error terms are assumed to be distributed independently of each other, which is a strong assumption given the usual autocorrelation structure of time series variables, such as prices and transaction costs. Regime 1 has a normal distribution $f_{t}^{1}$, and regimes 2 and 3 have distributions $f_{t}^{2}$ and $f_{t}^{3}$, respectively, which are mixtures of a standard normal and a half-normal distribution. ${ }^{18}$

\footnotetext{
${ }^{18}$ The distribution function of such a composite density was derived by Weinstein (1964). Other choices of distributions are, of course, also possible as, e.g., in Yang and Kant (2008). In the following,
} 
Hence, the distributions of the regimes are as follows:

$$
\begin{aligned}
f_{t}^{1} & =\frac{1}{\sigma_{e}} \phi\left[\frac{R_{t}-\delta}{\sigma_{e}}\right] \\
f_{t}^{2} & =\left[\frac{2}{\left(\sigma_{e}^{2}+\sigma_{u}^{2}\right)^{1 / 2}}\right] \phi\left[\frac{R_{t}-\delta}{\left(\sigma_{e}^{2}+\sigma_{u}^{2}\right)^{1 / 2}}\right]\left[1-\Phi\left(\frac{-\left(R_{t}-\delta\right) \sigma_{e} / \sigma_{u}}{\left(\sigma_{e}^{2}+\sigma_{u}^{2}\right)^{1 / 2}}\right)\right] \\
f_{t}^{3} & =\left[\frac{2}{\left(\sigma_{e}^{2}+\sigma_{v}^{2}\right)^{1 / 2}}\right] \phi\left[\frac{R_{t}-\delta}{\left(\sigma_{e}^{2}+\sigma_{v}^{2}\right)^{1 / 2}}\right]\left[1-\Phi\left(\frac{\left(R_{t}-\delta\right) \sigma_{e} / \sigma_{v}}{\left(\sigma_{e}^{2}+\sigma_{v}^{2}\right)^{1 / 2}}\right)\right]
\end{aligned}
$$

where $\phi(\bullet)$ and $\Phi(\bullet)$ denote the standard normal density and distribution function, respectively. $\sigma_{e}, \sigma_{u}$, and $\sigma_{v}$ are the standard deviations of the error terms $e, u$, and $v$. Barrett and Li (2002) introduced $\delta$, which measures unobserved transaction costs (see (2.19)).

The resulting distribution is therefore a mixture of one standard normal and two mixture distributions

$$
f_{t}\left(R_{t} \mid \theta\right)=\lambda_{1} f_{1}+\lambda_{2} f_{2}+\lambda_{3} f_{3}=\sum_{l=1}^{3} \lambda_{l} f_{l}
$$

subject to the conditions on the weights

$$
\begin{aligned}
\lambda_{l} & \geq 0 \\
\sum_{l=1}^{3} \lambda_{l} & =1
\end{aligned}
$$

where $l=1,2,3$ for a three-regime model. Examples of empirical mixture distributions are shown in Figures 2.9 and 2.10 below. The likelihood of the sample of the basic model is the product of the weighted average

$$
L(\theta)=\prod_{t=1}^{T} f_{t}
$$

These joint probabilities can also be thought of as representing a decision rule for the classification of observations into regimes. However, the dynamics and interdependencies which typically characterize price data are not regarded, and neither does the model allow for the specification of the RGP (the stochastic process which induces the regime switches and generates the regimes). Hence, the estimation of measures similar to the adjustment speed of cointegration models is not possible (see Figure 2.11, p.

we drop the index $A B$ which denotes the respective market pair because it is clear that the analysis always focuses on pairs. 
CA-US trade

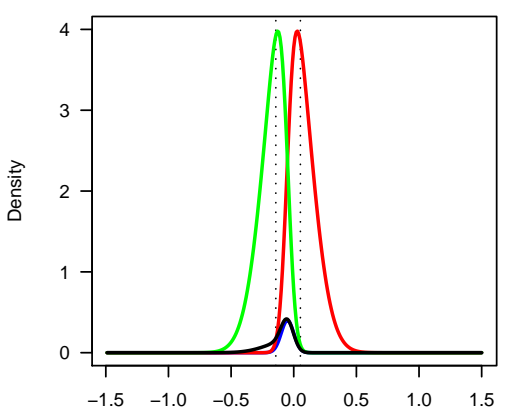

CA-US no trade

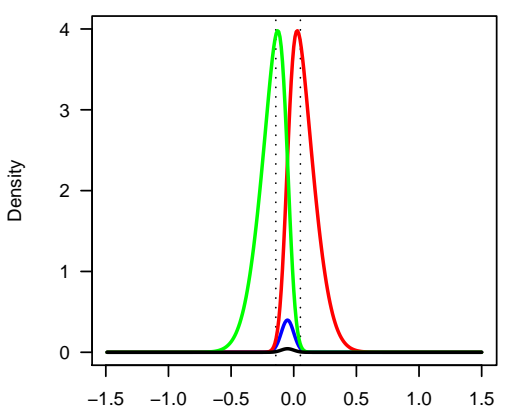

US-JP trade

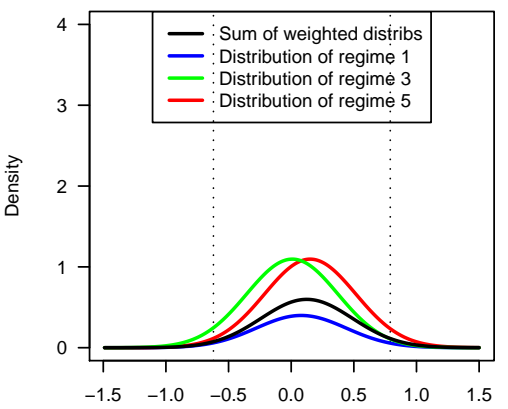

US-JP no trade

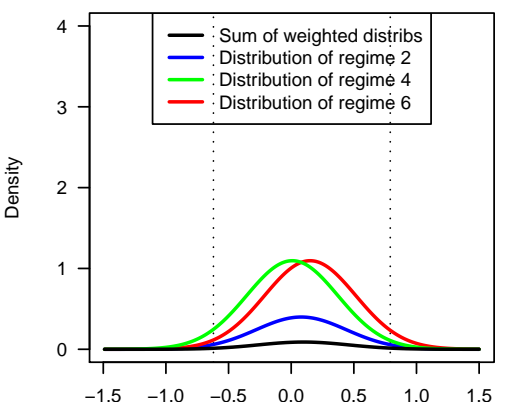

JP-US trade

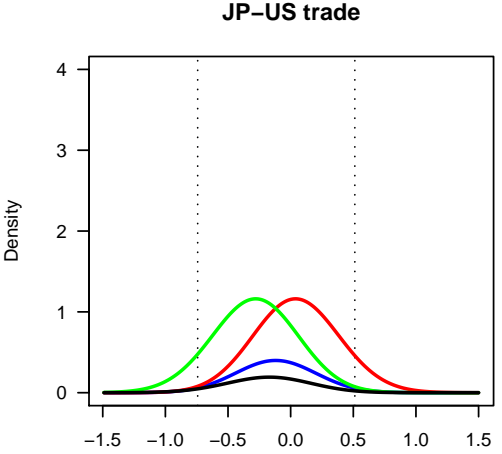

JP-US no trade

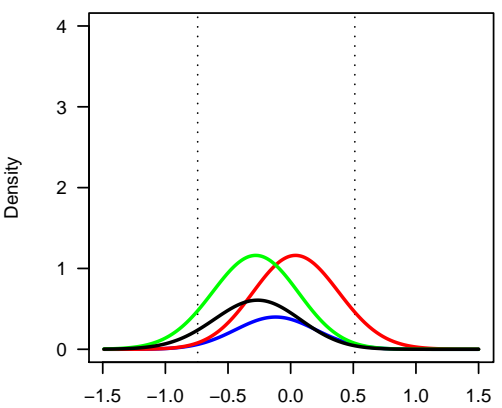

Note: These examples are calculated for selected trade directions based on the model and the estimation results of Barrett and Li (2002, Table 4). Baulch (1997) does not provide all estimated parameters so that the densities cannot be reconstructed. The vertical dotted lines denote the parity bounds, that is, the $95 \%$ confidence interval around $\delta$ based on the estimated variance of the error term of regimes 1 and 2 , respectively. These distributions are unweighthed in the sense that they are not yet multiplied by the estimated weights $\hat{\lambda}_{l}$.

Figure 2.9: Examples of Unweighted Estimated Mixture Distributions

56). In other words, it provides probabilistic statements on regime incidences and frequencies but remains silent on the potentially underlying mechanism. Obviously, this characteristic can be regarded as a strength of the model class, since the RGP cannot be misspecified, and it thus leaves flexibility for the application of the model to different settings.

\section{Extensions}

A currently vivid field of research is the extension of the model's flexibility which yielded several publications in the last decade. Barrett et al. (2000) and Barrett and $\mathrm{Li}$ (2002) introduce six regimes which allow to distinguish between market efficiency and market integration. ${ }^{19}$ Moreover, they account for unobserved transaction costs in the model. Park et al. (2002) suggest considering time-dependent parameters. The dependence is modeled as exogenously determined discrete shifts in parameters, which correspond to policy regimes. Negassa and Myers (2007) generalize this approach by estimating the end of the transition and allowing for a gradual change in parameters.

19 Their understanding of MI differs from the definition stated in Section 1.3.4, p. 16. They regard it as tradability without considering the dimension of information flow, which, in our opinion, is not identical to "price-based notions of efficiency" (Barrett, 2001). 

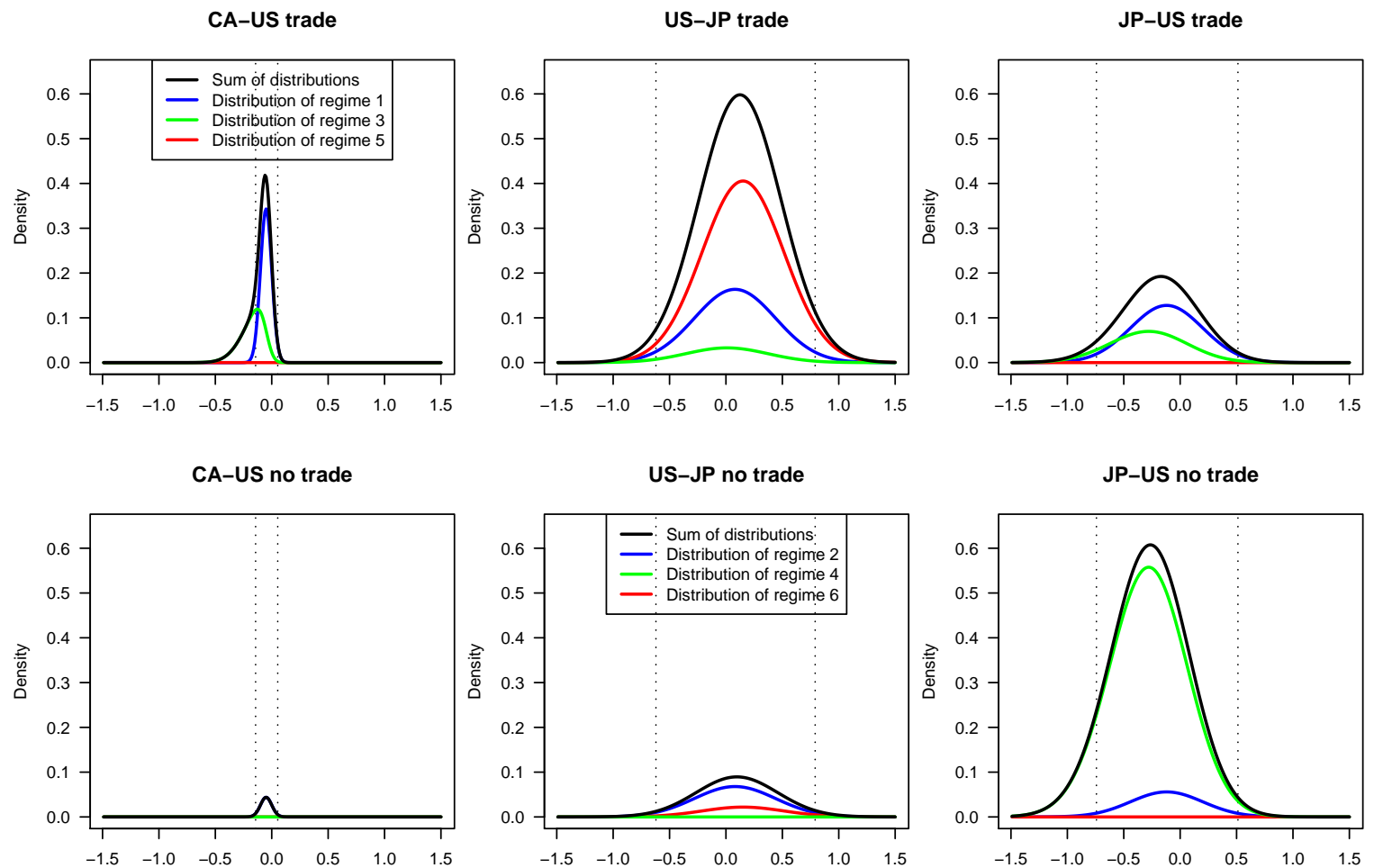

Note: The resulting mixture distributions (black lines) as the sum of the weighted regime distributions are identical to the black lines in Figure 2.9, only the scales of the diagrams differ. The weights $\hat{\lambda}_{l}$ of each of the regime distributions are the estimation results from Barrett and Li (2002, Table 4). The vertical dotted lines denote the parity bounds, that is, the $95 \%$ confidence interval around $\delta$ based on the estimated variance of the error term of regimes 1 and 2, respectively.

Figure 2.10: Examples of Weighted Estimated Mixture Distributions

\subsubsection{Estimation}

Estimation of the PBM is straightforward via maximum likelihood. The log-likelihood $\ln L(\theta)$ in (2.28) is maximized conditional on the above mentioned constraints, so that the estimates $\hat{\theta}=\left(\hat{\delta}, \hat{\sigma}_{e}, \hat{\sigma}_{u}, \hat{\sigma}_{v}, \hat{\lambda}_{1}, \hat{\lambda}_{2}, \hat{\lambda}_{3}\right)^{\top}$ are obtained. However, the model in its current form is only capable of analyzing market pairs. Thus, it has to be estimated for each meaningful combination of markets. Moreover, the estimation has to be carried out for each trade direction separately since transaction costs, and thus rents to spatial arbitrage, seem plausible to depend on the direction of trade flow, e.g., freight rates or tariffs (Barrett and Li, 2002, p. 297). Hence, up to $2\left(\begin{array}{c}n \\ 2\end{array}\right)=\frac{n !}{(n-2) !}$ estimations have to be carried out for $n$ markets. Most studies opt for monthly data as the adequate frequency to be considered.

\subsubsection{Interpretation}

The model provides probabilistic results similar to the ones of the MSVECM. The PBM gives immediate estimates of regime probabilities, which are often interpreted as frequencies of regime incidence. This seems to be a reasonable way of interpretation 


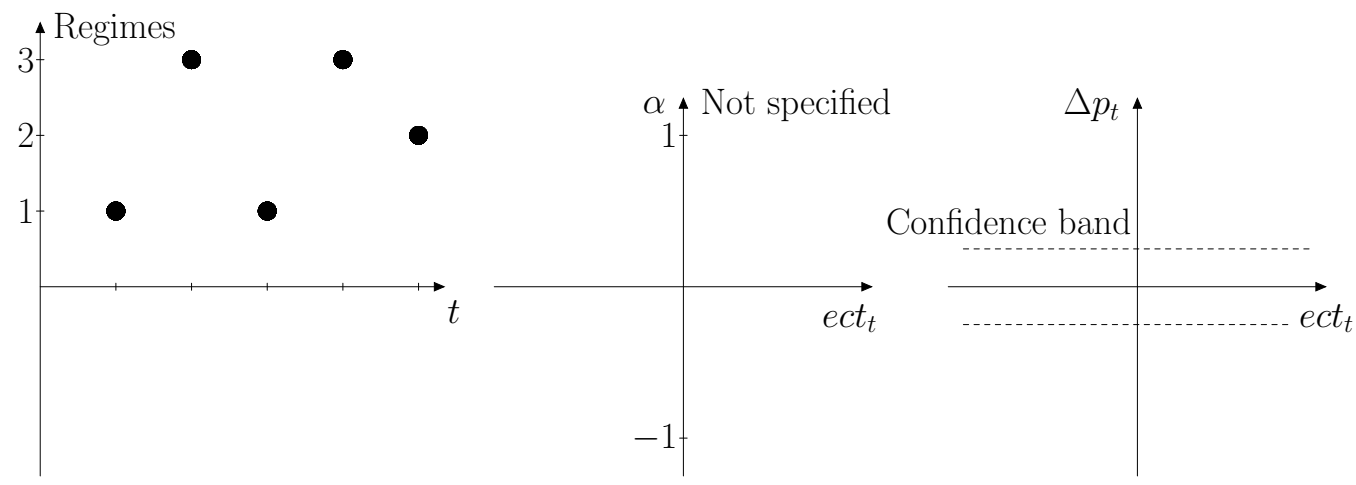

Figure 2.11: Characteristic Graphs of a Typical PBM

because it acknowledges the uncertainty connected with the regime assignment. From these estimates, conclusions regarding the occurrences of various market conditions can be drawn as, e.g., in Barrett and Li (2002). Furthermore, the probabilities of the incidence of each regime can be calculated for each time period. Based on the estimated standard error $\hat{\sigma}_{e}$ of regime 1, the parity bounds around the transaction costs can be calculated as the $90 \%$ or $95 \%$ pointwise confidence band, and then plotted against the price differences (right panel of Figure 2.11). ${ }^{20}$ The bounds reflect the fact that the observed or predicted transactions costs which were used in the estimation might contain a considerable level of uncertainty. The estimated parity bounds can be seen to represent the range of transaction costs in which these costs equal price differences, that is, in which the rents to spatial arbitrage equal zero, with $95 \%$ probability. Based on the estimates, a number of insightful summary measures can be calculated. Barrett and Li (2002) suggest, for example, moving averages, empirical conditional distributions, and bootstrapped descriptive statistics of the expected percentage returns to spatial arbitrage. Furthermore, distributions of particular market pairs and/or market conditions can be reconstructed based on the estimated parameters; see, e.g., Figures 2.9 and 2.10 .

Nevertheless, the direction-specific estimation leads to the problem that the estimates $\hat{\theta}$ for both trade directions might not be unique, which poses challenges for meaningful interpretation. This means that the estimated distributions for both directions, which serve as the classification rules, can differ. Consequently, the measurements of the market conditions also may not be unique. Barrett and Li (2002) try to tackle this issue by defining probability ranges for some conditions. The estimated ranges are mostly very narrow, but cover up to 50 percentage points or more in other cases. However, this characteristic complicates interpretation of the model to a considerable extent.

\footnotetext{
${ }^{20}$ However, it might be worthwhile to consider simultaneous confidence bands instead.
} 


\subsubsection{Applications}

The PBM is applied in agricultural price analysis much less frequently than cointegration methods are. Most applications assess market conditions in space with the exception of Yang and Kant (2008), who assess vertical relationships in the Canadian wood pricing system. Baulch (1997) and Moser et al. (2009) study rice markets in the Philippines and Madagascar, respectively. Barrett et al. (2000) and Barrett and Li (2002) analyze pork and soybean meal markets of the Pacific Rim. Padilla-Bernal et al. (2003) assess US-Mexican tomato trade. They predict transaction costs by allowing for seasonal and regional effects in these costs. Tostão and Brorsen (2005) examine regional maize markets in Mozambique, while Cirera and Channing (2008) study the effects of changing infrastructure on these markets. Brümmer et al. (2004) analyze regional wheat markets in Ukraine. Park et al. (2002) and Negassa and Myers (2007) focus on the effects of changing policy on Chinese and Ethiopian grain markets, respectively. 



\section{Summary}

We believe that each model has its particular merits and weaknesses. The point of view we would like to follow here is expressed in the quote attributed to the great statistician George E.P. Box:

Essentially, all models are wrong, but some are useful.

(Box and Draper, 1987, p. 424)

This rightly expresses our view that there are no "right" or "wrong" models. Instead, there are more vs. less adequate models for answering a particular empirical question. While Chapter 2 focuses on the intuition and the merits of each model class, we now would like to provide a critical discussion. Although researchers tend to emphasize the merits of a certain approach and to understate its weaknesses, we believe that it is worth it to keep its weaknesses in mind as well in order to comprehensively assess the research results. Thereby, we focus on selected issues which we consider most relevant and elaborate on them in a bit more detail. ${ }^{1}$ Among other issues, we reflect upon the reconcilability of the two model families dealt with in this dissertation, which are model classes based on cointegration analysis versus mixture distributions.

\subsection{Cointegration Models}

The statistical theory of the basic class of cointegration models, i.e., the VECM, is well-established and undisputed. Scientists who considerably advanced this area are Clive Gran-ger, the Copenhagen School of Economics with scientists such as Søren Johansen or Katarina Juselius, Pennti Saikkonen, Helmut Lütkepohl, and many others. However, there are disputes which are focused on particular applications of the econometric model class, e.g., the application of cointegration models to spatial and vertical price analysis. In agricultural economics, publications critical of the application of this model family to PT and MI analysis are, e.g., Barrett (1996), McNew and Fackler (1997), Miljkovic (1999), Barrett et al. (2000), Miljkovic and Paul (2001), Barrett and Li (2002), or Balcombe and Morrison (2002). ${ }^{2}$

\footnotetext{
1 We compare the models based on their the currently existing versions and the thoughts developed in Chapter 2 and suggest some points for possible extensions. However, future developments, e.g., might bring the two model families closer together by improving the weaknesses of one in comparison to the other.

2 However, since the VECM is not the focus of this thesis, we do not discuss these criticisms in detail here but address problems of models for nonlinear PT. The interested reader is referred to the
} 


\section{TVECM}

Saikkonen (2008, p. 295 ) notes that "threshold error-correction models introduced by Balke and Fomby (1997) have probably been the most popular nonlinear errorcorrection models considered so far, and recently their smooth transition counterparts have also attracted attention."

For the TVECM class, it is usually assumed that, besides the adjustment speeds, the autoregressive dynamics show nonlinearities as well, i.e., regime-dependent behavior. In applied research, econometric approaches to obtain evidence on this question of model specification are rarely employed. We believe that the distinction between the $\mathbf{X}$-form and the $\mathbf{R}$-form of cointegration models is a powerful tool in this context. Juselius (2008, ch. 9) points out the usefulness of a number of recursive tests for assessing the stability of various parameters or parameter combinations. In the context of nonlinear PT, they seem particularly useful since they can offer manifold indications on which of the parameters (see Table 1.1) are likely to exhibit nonlinearities. Moreover, estimation of the model proceeds in two steps in most studies, in which the long-run equilibrium relationship is usually estimated using the Engle-Granger OLS method. However, the approach of Johansen (1988) which introduced the $\mathbf{R}$-form has been shown to be superior in most cases as discussed in subsection 1.3.3, pp. 12 .

Furthermore, the decision between a Band- and an EQ-TVECM as the more appropriate model specification in a three-regime TVECM is mostly left to the subjective choice of the researcher. A few publications, such as Mancuso et al. (2003) and Serra et al. (2006a) suggest an approach to obtain evidence on this question. Gaul (2008, sec. 2.4, pp. 44), however, develops a test for the cointegration rank in each of the regimes. Both methods are limited to TVECMs of only a single cointegration relationship which is also the specification exclusively used in PT analysis up to now. The selection of a particular specification of the many possibilities offered by the TVECM class could be achieved via approaches such as the SPVECM. First, the nonlinear adjustment can be flexibly estimated from the data. Plots of the nonparametric estimates can then suggest a suitable specification of the TVECM, that is, whether two or three regimes are most adequate, similarly as in, e.g., Escribano (2004, p. 94). Second, it allows for more than one cointegration relationship and hence for dimensions of the loading matrix $\alpha$ of more than one column. The TVECM in its current form only allows for one cointegration relationship and is most frequently applied to bivariate analyses. A model class such as the SPVECM allows for more than two variables to be simultaneously regarded. On the other hand, the approach is limited by the curse of dimensionality often found in the context of semi- or nonparametric estimation. However, empirical analyses rarely study more than five or six markets.

mentioned publications. 


\section{MSVECM}

The MSVECM has a number of merits. For example, it is applicable to multivariate settings analyzing more than two variables. Its central property is that the determinants inducing regime switches are allowed to be latent. However, this poses challenges regarding the interpretation of the estimated regimes to the researcher. The TVECM requires the a priori specification of the variable(s) causing the regime-dependent behavior. In the case of the MSVECM, the task is left for a posteriori interpretation. The model provides evidence on the number of regimes and timings of switches which can be interpreted based on a sound knowledge of the market under study by connecting it, e.g., to known market events. While information on the modeled RGP might already permit interesting insights into market dynamics, the applied researcher is typically interested in the characterization of the regimes and in finding a plausible interpretation for the regime switches.

At this point, an interesting duality between the TVECM and MSVECM classes appears. While the variables suspected to cause regime switches have to be specified a priori, that is, before decision rules and regime assignments are estimated conditional on this choice in the case of the TVECM, regime incidences are estimated first in the context of the MSVECM. Potential determinants which caused the switching can only be identified a posteriori in the latter case. This illustrates that both model classes can lead to strongly differing results regarding regime incidences and thus parameter estimates. The MSVECM identifies regimes based on the dominant data-inherent switches. However, the regimes of the TVECM depend on the choice of the index and its importance among the manifold determinants potentially causing regime-dependence in the data. ${ }^{3}$

\section{SPVECM}

The core property of the SPVECM is nonparametric modeling of the disequilibrium adjustment, which is the reason for the model's flexibility. However, this feature might also be a disadvantage in some contexts since only graphs can be produced from the estimated smooth functions which, e.g., complicates testing considerably. While the influence of the chosen kernel function $K(\bullet)$ is negligible, the choice of the bandwidth $h$ is crucial. It governs the smoothness of the estimate, but also its bias and variance. A number of approaches have been developed in the last decade or so which enable an optimal bandwidth choice. Although the model class allows for any multivariate setting analyzing more than two price series connected by more than one cointegration relationship, it is limited by the curse of dimensionality.

\footnotetext{
3 This consideration is based on the thought that empirical data might be subject to a number of impacts causing regime-dependence, e.g., in short-run PT. Different sources of nonlinearities might interfere with each other. Therefore, the questions comes up what is the dominant source among the (potentially) manifold impacts. General statements on the composition of such influences can hardly be made since it varies from case to case. The longer the time series analyzed are the more likely such a problem seems.
} 


\section{The Regime-Generating Process}

Balke and Fomby (1997, p. 632) remark that "cointegration is a global characteristic of the time series while the threshold regimes are local characteristics". Hence, the equilibrium error may behave locally, i.e., inside the middle regime of the typical specification of the TVECM, as unit root process, although the series globally appears to be stationary. Consequently, we conjecture that any RGP might be suitable for the cointegration family of nonlinear PT models that yields a globally stationary process. In other words, we conjecture that a suitable RGP has to restrict the global variance of the equilibrium error.

Examples of suitable RGPs which restrict the variance of the equilibrum error were discussed in Chapter 2. Figures 3.1, 3.2, and 3.3 illustrate this property. They depict the estimated mean and variance for 10000 simulations of a Band-TVECM, an EQ-TVECM and a MSVECM, respectively. Even though in each of the TVECM simulations, the time series has a unit root in the middle regime (i.e. locally), the thresholds have the effect of stabilizing the variance of the model once the time series has crossed them (globally). This is achieved by the fact that error-correction takes place once the middle regime is left in either direction. The series are pulled back either to the edges of the threshold band in the case of the Band-TVECM or to the long-run equilibrium itself in the case of the EQ-TVECM.

Figures 3.1 and 3.2 demonstrate that the variance of a Band-TVECM is considerably larger than the variance of a EQ-TVECM of identical parameters even if the EQ-TVECM does not have adjustment in the middle regime. This illustrates that equilibrium errors are more strongly adjusted by the EQ-TVECM than by the BandTVECM. Hence, it indicates that the global variance of a regime-dependent VECM is likely to be a complex function of, e.g., the chosen RGP, the parameters of the model, and the variance of the innovations. A number of other suitable RGPs could be thought of. ${ }^{4}$ However, certain RGPs of a TVECM or a MSVECM may not globally restrict the variance. An example of an improper RGP is a unit root in one of the outer regimes of a three-regime TVECM since this would leave the variance unbounded from one direction.

\footnotetext{
4 For graphical representations of further examples, see, e.g., Escribano and Pfann (1998, Figure 2), Escribano (2004, Figures 3-6), or Meyer (2004, Figure 1).
} 
100 and 5 of 10000 realizations for 250 and 500 periods, resp.

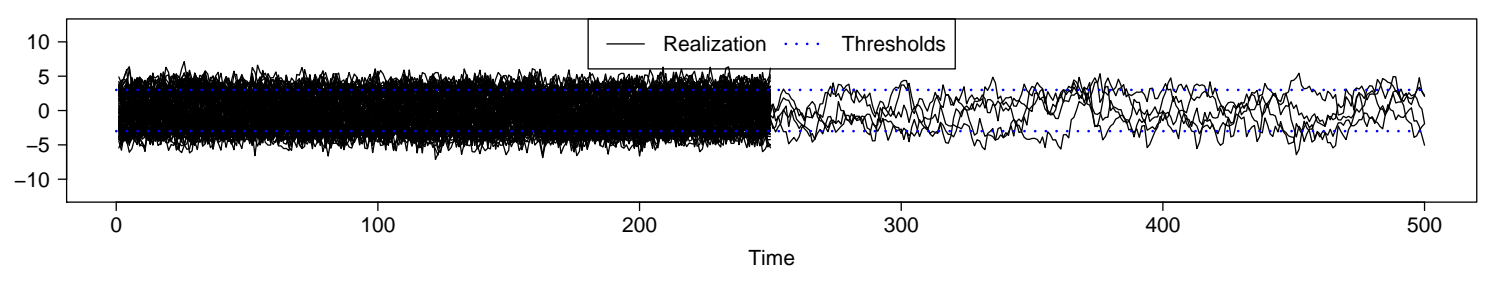

Mean of 10000 realizations for each time point

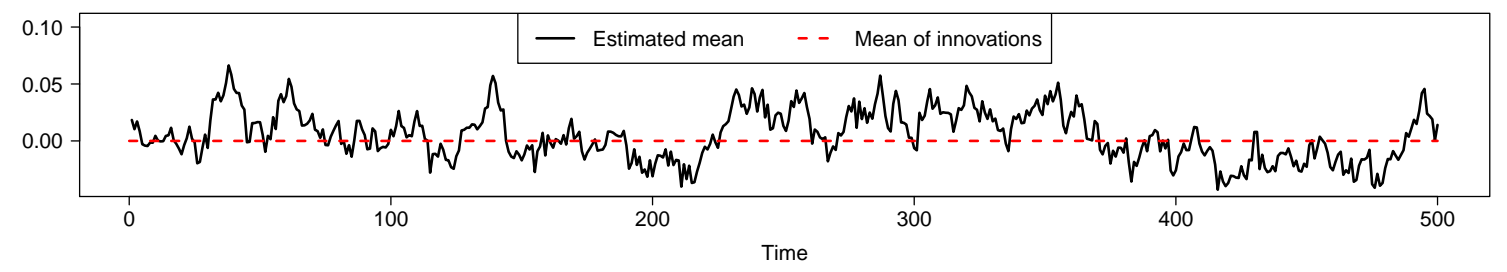

Variance of 10000 realizations for each time point

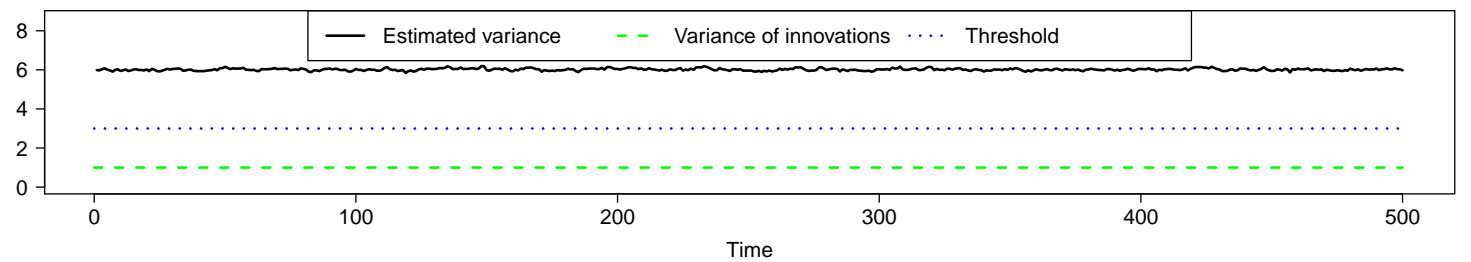

Note: The estimated mean and variance are based on 10000 realizations of a MSVECM with identical parameters as the one in Figure 2.1, p. 31. Only the innovations are repeatedly randomly generated. "Mean" and "variance" of innovations refers to the innovations of the cointegration relationship $\epsilon_{t} \sim N I D(0,1)$ (see Figure 1.3, p. 9).

Figure 3.1: Mean and Variance of a Band-TVECM

\subsection{Parity Bounds Models}

The PBM implicitly models nonlinearities in short-run PT in the sense that the observations are classified into various regimes which correspond to differing market conditions, that is, to differing behavior of economic agents in the short run. The underlying regimes are derived from economic theory and are even able to distinguish between market efficiency and market integration in some model versions. ${ }^{5}$ However, the approach, in its current form, only focuses on the results of economic agents' activities and does not enable econometric modeling of the process which might have led to this result empirically observed. For example, Barrett and Li (2002, p. 297) note that it is "ill-suited to answering questions about the speed or extent of convergence to tradability or equilibrium." On the other hand, this can be regarded as an advantage since the researcher never can be sure about the true RGP, so that not specifying it prevents from misspecification. However, an appropriately chosen RGP can offer interesting additional insights into price relationships.

$\overline{5}$ Market integration is particularly defined in this context, see, e.g., Barrett and Li (2002). 

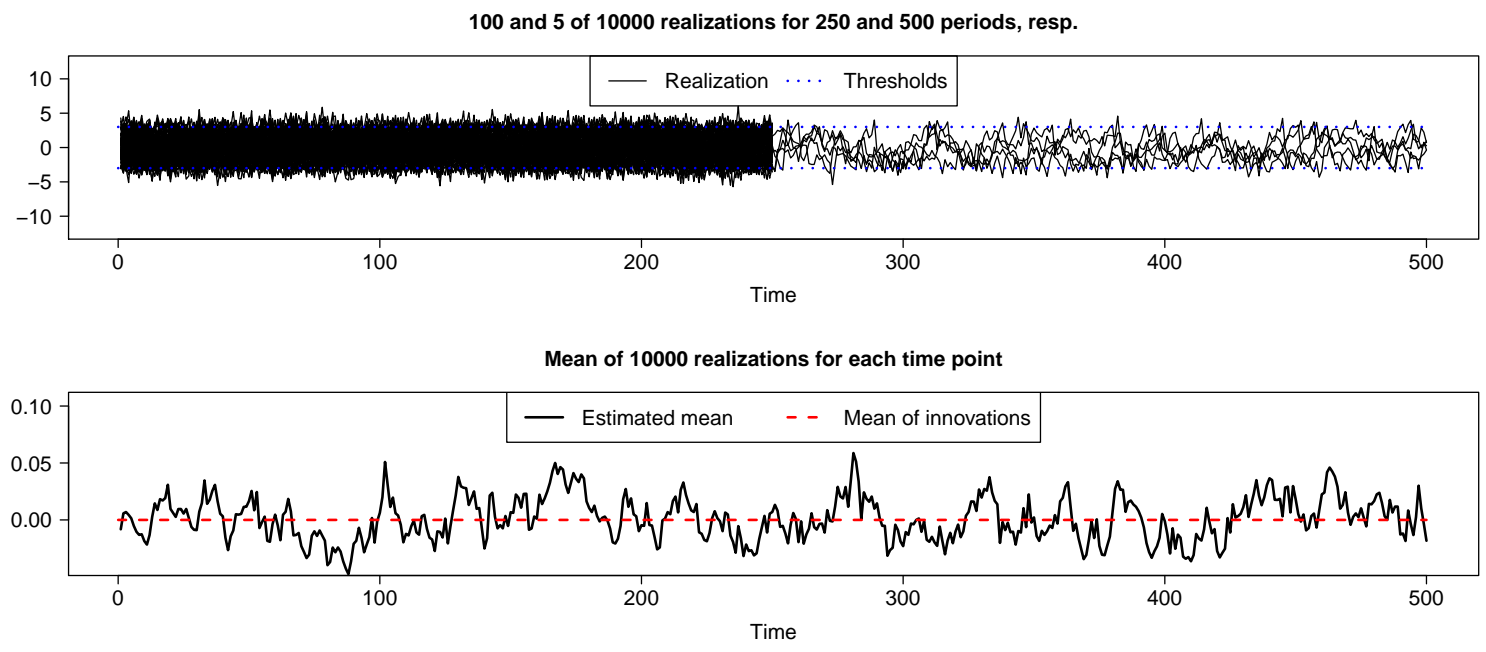

Variance of 10000 realizations for each time point

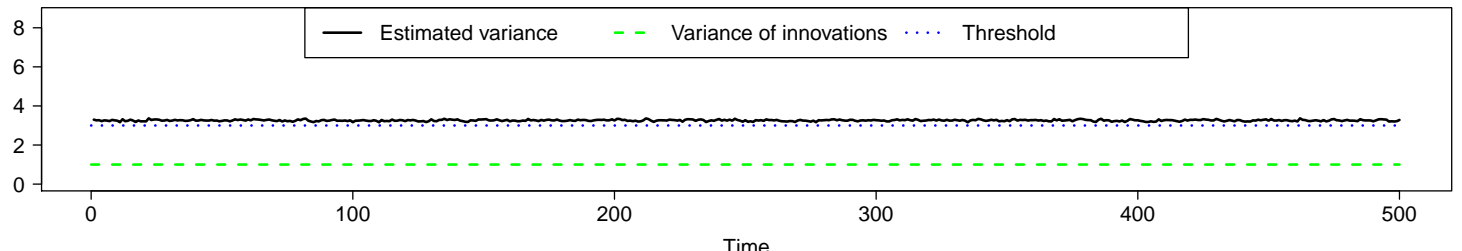

Note: The estimated mean and variance are based on 10000 realizations of a MSVECM with identical parameters as the one in Figure 2.2, p. 32. Only the innovations are repeatedly randomly generated. "Mean" and "variance" of innovations refers to the innovations of the cointegration relationship $\epsilon_{t} \sim N I D(0,1)$ (see Figure 1.3, p. 9).

Figure 3.2: Mean and Variance of an EQ-TVECM

Baulch (1997) notes, that the approach is inherently static, although various recent extensions have added some dynamic aspects. The researcher has to subjectively decide for distributions which she considers adequate in the context at hand. ${ }^{6}$ This criticism is often brought forward as a major drawback of the model. Another assumption is that the quantities modeled, usually the rents to spatial arbitrage, are thought of as being independent draws from distributions which are identical over time. We, however, consider this latter assumption to be the more restrictive one because it implies that the rents are independent of their past. In other words, economic agents are assumed to discard all information of the past for their actions in the current period. Furthermore, positive rents in one period can be randomly followed by positive, zero or negative rents in the next period (as schematically displayed in the left panel of Figure 2.11, p. 56). This means that net profits of a trader may randomly be followed by net profits, zero profits or net losses in the next period. Such a strong assumption of memoryless behavior is hardly compatible with the behavior of economic agents one would expect, particularly from traders. We conjecture that besides the equilibriumrestoring forces, e.g., of the exploitation of positive rents to spatial arbitrage, also the "stickiness" of economic variables (autocorrelation) such as prices should be regarded.

$\overline{6}$ We will briefly address the question of distributions in the following section. 
100 and 5 of 10000 realizations for 250 and 500 periods, resp.

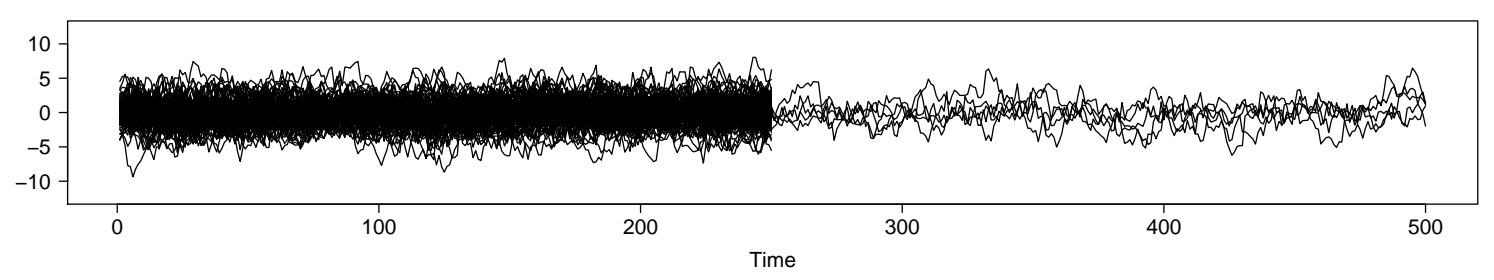

Mean of 10000 realizations for each time point

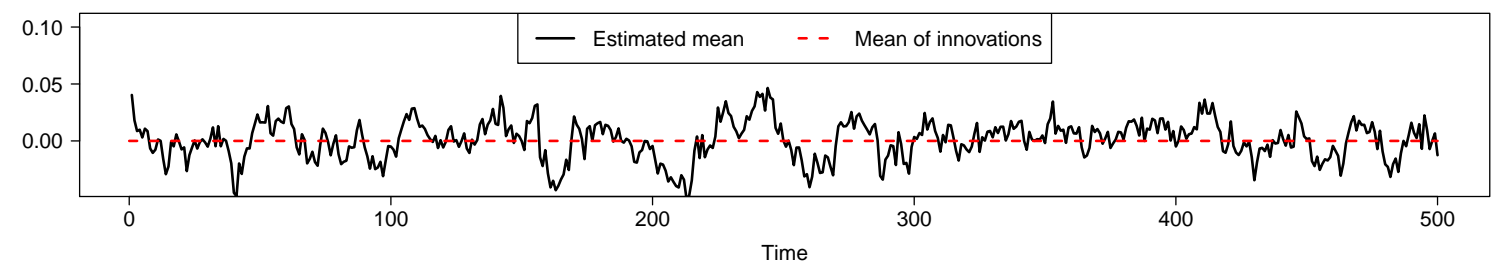

Variance of 10000 realizations for each time point

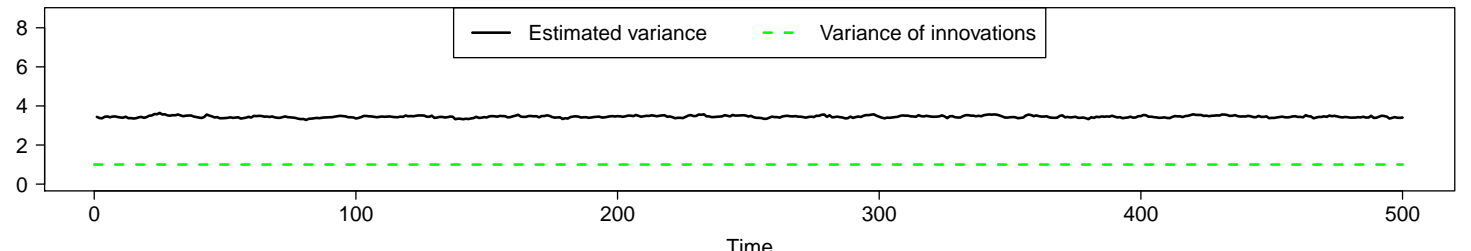

Note: The estimated mean and variance are based on 10000 realizations of a MSVECM with identical parameters as the one in Figure 2.7, p. 41. Only the innovations are repeatedly randomly generated. "Mean" and "variance" of innovations refers to the innovations of the cointegration relationship $\epsilon_{t} \sim N I D(0,1)$ (see Figure 1.3, p. 9).

\section{Figure 3.3: Mean and Variance of a MSVECM}

However, we are not aware of publications which analyze properties and dynamics of rents to spatial arbitrage in the time domain in order to obtain empirical evidence on such properties.

Another strong argument against the assumption of memoryless behavior is the durarion of physical trade. Considerable time lags are occurring in agricultural trade since several weeks usually pass between the first planning and collection of information and the completion of the physical commodity movement. Incentives in one period, in the form of positive rents to spatial arbitrage, will rarely trigger immediate response of economic agents; neither is it likely that the arbitrage process will be finished in the same period. The PBM literature is aware of this fact; that is why most authors recommend the use of monthly data. Although this can be considered to be a reasonable period for agents' response, it still implies "memorylessness on a monthly basis," which might not hold for every product in every context. The underlying problem is the appropriate frequency of the discretization of continuous time. That is, what is the appropriate time interval for the economists' flashlight to bring light into the darkness of reality? ${ }^{7}$ We note that for some variables such as GDP growth the inter-

7 That is, how frequently should she point her flashlight to economic variables in order to measure 
vals are much longer - one year or more - but their realizations are still not considered to be independent of each other. This question remains unresolved for the PBM. Approaches such as the Frisch-Waugh-Lovell Theorem, p. 10, might provide one step in this direction. It would allow the cleaning of the rents, at least from short-run autoregressive dynamics, although the time dependence of economic incentives would still not be addressed.

Last, the PBM is restricted to trade-flow driection specific analyses because it is based on direction-specific transaction costs. Asymmetric costs of trade seem plausible in most cases since, e.g., freight rates from urban to rural regions might differ from rates from rural to urban regions, etc. This restriction means, first, that only bivariate pairs can be studied and, second, that in the case of bidirectional trade between two markets, one estimation for each direction has to be carried out. Hence, non-unique parameter estimates are obtained as, e.g., in Barrett and Li (2002), which poses considerable challenges to meaningful interpretation. It has severe consequences because the four market conditions defined by the authors turn out to be trade-flow direction specific instead. If they would apply for a system of at least two markets without being direction-specific, the associated probabilities of all four conditions should add up to one for this system. However, in the current form of the model they only add up for each direction of trade. Barrett and Li try to translate the conditions estimated per trade-flow direction into direction-independent conditions for a market pair. However, the sums appear to be quite far from unity for a number of market pairs. ${ }^{8}$ This indicates that a measurement of the reasonably defined market conditions for market pairs (market systems) is not as straightforward as it seems in the context of the PBM.

them?

8 See Barrett and Li (2002, Table 5). For example, the sums of the market conditions' probabilities of the pairs Canada-Taiwan and U.S.-Japan range between 0.95 and 1.21, and 0.64 and 1.48, respectively. 


\subsection{Parity Bounds vs. Cointegration Models}

In this section, we aim at comparing and contrasting both model families. While we will elaborate on merits and disadvantages of both methods relative to each other, we will try to demonstrate similarities and correspondences between them as well.

Both model families, although mainly applied to spatial price analysis, are also applicable to studies of vertical price relationships along the processing chain. For example, Yang and Kant (2008) study wood price in Ontario using a PBM. Ben-Kaabia et al. (2005) and Ben-Kaabia and Gil (2007) analyze vertical price relationships in Spanish poultry and lamb markets, respectively, by employing a TVECM. Brümmer et al. (2009) assess price relationships between wheat and flour in Ukraine using a MSVECM.

The fundamental difference between both families consists in the fact that cointegration models focus on the time series dynamics of price data. They even allow the distinction between long- and short-term interdependencies conditional on the existence of a stationary linear combination of the price series considered, which is interpreted as the long-run equilibrium. The PBM family, in its current form, is not able to account for any autocorrelation in price series. It is even explicitly assumed that the observations of the time series studied are realizations of independent and identically distributed random variables. It focuses on the flexible modeling of the results of such dynamic processes instead. Furthermore, there are indications that the currently mostly used estimation approaches of the PBM as well as of the TVECM and MSVECM classes encounter problems in some cases, see, e.g., Barrett and Li (2002, p. 297) or Appendix DII, pp. 201.

Despite the fact that the PBM uses at least as much information as cointegration models do, less information in terms of estimation results is obtained. Cointegration methods often only use prices but retrieve information regarding three aspects: dynamics and interdependencies of price series in time; the RGP, i.e., the stochastic model of the regime switching mechanism; and the regime classification. The PBM, in contrast, uses prices, transaction costs, or a number of variables with which transaction costs are predicted, and in some specifications also trade flow information. It therefore has much higher data requirements than the cointegration methods. On the other hand, this model class evaluates only one of the three aspects which cointegration methods provide, namely the classification of observations into regimes. The evolution of prices over time, their dynamics and interdependencies, and the modeling of the regime switching process are completely neglected by the PBM. However, it has the advantage of avoiding misspecification since it does not address these issues. Particularly in light of the existing rather vague model selection strategies for cointegration methods, this might be regarded a strength of the approach. In the end, the researcher has to balance her interests and subjectively decide which of the models is most adequate for the problem and the data to be analyzed, based on her experience. 
The rents to spatial arbitrage as the difference between the price difference and transaction costs and the equilibrium errors of cointegration models correspond to each other in some aspects but differ considerably in others. Transaction costs are estimated in both model families, while the underlying information differs. Transaction costs are implicitly estimated by the constant of the long-run relationship in case of the VECM and the MSVECM or by the thresholds in case of the TVECM. Current versions of the PBM predict transaction costs based on a number of explanatory variables or by extrapolation of a single or a few observations of transaction costs. ${ }^{9}$ The latter approach promises more reliable estimates of real equilibrium prices since more information is used. On the other hand, it has the drawback that data requirements are considerably higher. For cointegration analysis, price series are sufficient for model input. To obtain sufficiently reliable predictions of transaction costs, a whole range of explanatory variables has to be available, which is rarely the case. In some instances, more data to be collected might also mean a considerably increased level of measurement error and hence uncertainty because transaction costs are a complex quantity, as discussed, e.g., in Barrett (2001), with a number of components which can hardly be correctly observed. It is therefore questionable whether the degree of uncertainty of the estimated transaction costs is significantly reduced by the PBM methodology. From an applied point of view, the issues of data requirements and the availability of reliable data can be key points for model choice and the performance of the analysis. Hence, the considerably larger information requirements of the PBM might limit the applicability of the model family to a large extent. ${ }^{10}$ Furthermore, the PBM uses price differences and thus corresponds to a cointegration model which imposes the restriction on the cointegration vector between two prices that $\beta=(1-1)^{\top} \cdot{ }^{11}$

In cointegration analysis, first a test for cointegration is conducted and then the equilibrium errors are estimated, because they would be meaningless quantities otherwise. However, the PBM methodology does not assess whether price series share a sta-

\footnotetext{
9 The amount of information used by the PBM is richer than in the case of cointegration models. That is, it conducts in the terminology of Barrett (1996) a level II or even, in the cases of Barrett and Li (2002) and Padilla-Bernal et al. (2003), a level III analysis. One might even state that Moser et al. (2009) conduct a level IV analysis since they use not only price, transaction cost, and trade flow data, but also a range of further variables to predict transaction costs, and hence the information content of this research is even richer.

${ }^{10}$ This can be seen, e.g., in the fact that the six-regime PBM introduced by Barrett et al. (2000) has only been applied, to our knowledge, in two more publications, Barrett and Li (2002), and PadillaBernal et al. (2003) over the past ten years. Recent analyses using the PBM only employ three-regime models.

${ }^{11}$ For the VECM and the MSVECM, this restriction would mean that the model's constant represents the transaction costs. The constant is left unconstrained in this case. It thus represents the model's estimate of transaction costs between the markets if prices are in long-run equilibrium. If untransformed (logged) data is used in the estimation, it represents hence a constant additive (multiplicative) margin. For a TVECM as in (2.2), however, particular restrictions depend on the version of the model. Both, Lo and Zivot (2001) and Goodwin and Piggott (2001) also use price differences, that is, a cointegration relationship for which the constant is restricted to zero and the two prices have coefficients of 1 and -1 , respectively.
} 
ble relationship (apart from possibly common driving forces), which is the stationary cointegration relationship in the cointegration world. Consequently, price differences of two markets which are neither directly nor indirectly linked by third markets might, by chance, roughly equal the predicted transactions between them. The PBM would in such a case conclude a high incidence of perfect integration because most observations would be classified into the binding arbitrage condition regime, that is, regime 1 in $(2.23)$, p. 53 .

The understandings of the central notions such as MI differ considerably between the proponents of the competing model families. A key point which is often claimed by the proponents of the $\mathrm{PBM}^{12}$ is that the definition of the regimes is better anchored in economic theory and thus more accurate in contrast to cointegration methods. Another central point of critique connected with this issue is reflected in the statement of Barrett et al. (2000): "The core reason is that conventional market integration tests rely exclusively on price data, and so cannot shed light on the quantity-based concept of market integration nor can they provide reliable inference on equilibrium conditions that depend as well on transactions cost and trade flow data."

We are not convinced by these arguments because cointegration methods can easily be extended by regarding more information, for example on trade flows. A VECM with slight modifications can yield a classification of observations into four regimes. For example, Stephens et al. (2009) or Ihle et al. (2010) estimate a VECM for periods with and without trade. Such a model would be able to capture at least three of the market conditions mentioned in the following Table 3.1. ${ }^{13}$ In this case, the index only consists of a trade flow dummy variable. Observations can be classified according to the values of the dummy and the sign of the estimated equilibrium error. If the index additionally encompasses the equilibrium error, even parameter estimates for each of the four regimes can be obtained. This model would be a version of a central AVECM as outlined in Table 2.1, p. 30 .

The extension of a TVECM to six regimes corresponding to the six regimes of Barrett and Li's PBM is straightforward. A continuous Band-TVECM of three regimes of the typical form (2.2), p. 28, corresponds to a three-regime PBM. If the threshold variable is generalized to an index which consists of the ect and a trade indicator variable, i.e., a dummy variable indicating trade flows, then regime classification corresponding to a six-regime PBM is obtained. Such a model would have a more complex decision rule consisting of two thresholds for classifying the equilibrium error and one threshold for the trade flow indicator. ${ }^{14}$

\footnotetext{
12 See, e.g, Barrett et al. (2000), Barrett (2001), or Barrett and Li (2002).

${ }^{13}$ The first condition of perfect integration means in such a model where of one threshold, i.e., $\theta^{(1)}=\theta^{(2)}$, that the ect would equal zero. Since this almost never happens in practice, this market condition can rarely, if at all, be observed.

${ }^{14}$ Compare also the discussion on pp. 26.
} 
It appears straightforward to translate the PBM terminology to the "cointegration world" since the rents to spatial arbitrage and the equilibrium error are quantities which correspond to each other. Therefore, we regard the differing economic interpretation of central notions and the regimes of PBM models to not be an argument in favor of the PBM methodology since it is not a unique property of this model family. Other model families or model classes can be adapted accordingly to yield similar results. Table 3.1 shows the correspondence of market conditions which result from the six-regime versions of the discussed model classes.

\begin{tabular}{l|ll}
\hline Market condition $^{\mathrm{a}}$ & PBM $^{\mathrm{a}}$ parameters & TVECM $^{\mathrm{b}}$ parameters \\
\hline Perfect integration & $R_{t}^{A B}=0$ & $\theta^{(1)} \leq e c t_{t} \leq \theta^{(2)}$ \\
Imperfect integration & $a_{t}^{A B}=1 ; R_{t}^{A B} \neq 0$ & $a_{t}^{A B}=1 ;$ ect $_{t}<\theta^{(1)}$ or ect $_{t}>\theta^{(2)}$ \\
Segmented equilibrium & $a_{t}^{A B}=0 ; R_{t}^{A B}<0$ & $a_{t}^{A B}=0 ;$ ect $t_{t}<\theta^{(1)}$ \\
Segmented disequilibrium & $a_{t}^{A B}=0 ; R_{t}^{A B}>0$ & $a_{t}^{A B}=0 ;$ ect $t_{t}>\theta^{(2)}$ \\
\hline
\end{tabular}

Note: $a_{t}^{A B}$ denotes the trade flow indicator variable which takes the value 1 in period $t$ if trade flows between markets $A$ and $B$ were reported and 0 otherwise. The semicolon denotes that the conditions to its left and right have to be fulfilled.

a The terminology and the version of the PBM are the ones in Barrett and Li (2002).

$\mathrm{b}$ The TVECM is continuous and of the Band version. The cointegration relationship is restricted, that is, the constant is constrained to zero and the coefficients of the two prices to 1 and - 1 , respectively. The parameters mentioned in the table correspond to the ones in (2.2), p. 28. However, the index and the decision rule differ here. The index consists of both the trade indicator and the continuous equilibrium error ect. The decision rule is hence the composite. It consists of two thresholds classifying the equilibrium error and one threshold with respect to the trade flow indicator.

\section{Table 3.1: Correspondence of PBM and Cointegration Terminology}

The task remains to obtain consensus on the definition of the central concepts market integration and price transmission, which cannot be treated here. However, we observe that the PBM, since it neglects the dynamics of the price time series, does not offer any information on PT either in the long or in the short run as defined in section 1.3.4, p. 16. Barrett and Li (2002) clearly state their understanding of MI as mere tradability of a product for which trade flows represent a sufficient but not a necessary condition. Tradability is also indicated by traders' indifference on whether to trade or not when the price differential equals transaction costs. However, Moser et al. (2009) lack an explicit definition of market integration. Negassa and Myers (2007) explicitly state that they assess spatial market efficiency.

Although both model families are capable of classifying the observations into regimes and hence of providing estimates of regime frequencies, the natures of the regime assignments differ. The TVECM creates mutually exclusive subsets which assign the observations uniquely to one of the possible regimes. In the case of the PBM, this is not straightforward. For each period, each of the regimes occurs with certain probability, which is similar to the obtained evidence on regime incidences of the MSVECM where the observations are assigned to the most probable regime in each period. Barrett and $\mathrm{Li}$ (2002) provide informative summaries of the estimation results, which provide insight into a number of aspects of interest. However, since the PBM has to be estimated for each trade direction between two markets, the obtained probabilities for 
the above mentioned market conditions are, as discussed above, in general not unique (Barrett and Li, 2002, see Tables 2 and 5), which complicates interpretation.

One argument in favor of the probabilistic nature of the PBM results is that the assignment of observations to regimes is inherently subject to uncertainty. One could argue that such results better reflect the inherent uncertainty than (seemingly) unique and deterministic assignments as, e.g., in case of the TVECM do. However, it may facilitate interpretation in some contexts but may also complicate it in others. Cointegration models might potentially be extended in such a direction, of which we sketch here only the basic idea without addressing issues of estimation and inference in detail. Model classes of one threshold, such as the central AVECM (see Table 2.1, p. 30), might be extended to estimate a confidence interval for the threshold instead of a point estimate. Such an approach might be reasonable since neither prices nor transaction costs of and between two markets are unique. Each trader is likely to be faced with at least slightly different buying and selling prices, and costs of trade. Hence, exact point estimates obtained for the case that the equilibrium errors equal zero, that is, of the long-run equilibrium relationship, pretend accuracy, but are only averages. Probability bounds in the form of confidence statements regarding the location of the threshold might be more adequate instead. This seems to be an interesting field of future research since it would add some fuzziness to the sharp classification decisions created by decision rules currently used by specifications of the TVECM. Hence, the character of the current type of decision rules for the TVECM could be enriched by an interesting approach. Another consequence would be that the correspondence between some classes of cointegration models and the parity bounds approach would become even stronger.

\section{A Distribution View of Cointegration Models}

Figures 3.1, 3.2, and 3.3 plot in the upper panel simulated realizations of selected processes along time, which follow a Band-TVECM, an EQ-TVECM, and a MSVECM, respectively. Figures 2.9 and 2.10 plot empirical distributions of the PBM. We suggest a unifying perspective on both model families by looking at cointegration models from the mixed distributions perspective of the PBM. ${ }^{15}$ We believe that the view on cointegration models in terms of distributions might offer interesting insights into both model families.On the following pages, Figures 3.4 until 3.8 plot the distributions of simulated realizations of different specifications of the TVECM and the MSVECM. They indicate interesting patterns which, in some cases, considerably differ from the distributions assumed in the context of the PBM as plotted in Figure 2.10, p. 55, which are mixtures of normal and half-normal distributions. The distributions resulting from cointegration models are close to normal distributions for some cases, but also deviate strongly for others.

\footnotetext{
${ }^{15}$ Although cointegration models can be looked at from a PBM perspective, is the other way around not possible because the PBM does not model any time dynamics.
} 
As illustrated in Figure 3.4, the distribution of realizations of a Band-TVECM with weak equilibrium adjustment seems to be uniform in the middle regime (upper right panel), while the two outer regimes seem to be two half-normally distributed (upper left panel). A uniform distribution in the middle regime is plausible since the series have a unit root within this regime. This implies that the realizations are equally likely everywhere in this band because they do not have an attractor around which they occur with higher probability. The half normal distributions in the outer regimes are less plausible at first sight. Johansen (1995, Theorem A.11, p. 228) notes that the errorcorrection behaviour corresponds to an exponential distribution. Thus, we suppose that the distribution of the innovations $\epsilon_{t}$, which is standard normal in the performed simulations, dominates the distribution of the outer regimes if the adjustment towards the edges of the band is weak. That is, it superposes the exponentail distribution resulting from error-correction.Figure 3.5, in comparison, confirms the expectation that the stronger the adjustment, the less frequent the realizations switch to the outer regimes. Furthermore, the distributions in the regimes deviate more strongly from uniform and normal, respectively, as illustrated in the upper right and the two lower panels. In the outer as well as in the middle regime the kurtosis of the distributions increases which can be seen in the two panels to the right. Stronger adjustment implies that the process tends to return faster to the edges of the band and considerbaly overshoots into the band as depicted in the upper left panel where the density is decreasing from zero towards the thresholds. Hence, the variance of the process is smaller (compare Figure 3.1, p. 63).

Figure 3.6, in comparison with Figure 3.5, illustrates the fact mentioned above that an EQ-TVECM means stronger effective adjustment than a Band-TVECM with identical parameters. The reason is that deviations from the equilibrium are corrected towards parity instead of towards the edges of the band, that is, the dotted thresholds when they exceed the thresholds $\theta^{(1)}$ and $\theta^{(2)} \cdot{ }^{16}$ Consequently, the distributions of the regimes deviate stronger from standard distributions than for the Band-TVECM. Particularly, the outer regimes differ markedly from a normal distribution. These effects appear to be more pronounced the stronger the adjustment of the EQ-TVECM is, as shown in Figure 3.7. These effects are similar to the ones observed in Figure 3.5 in comparison with Figure 3.4, but even more distinct due to the differing adjustment mechanism. The increase in kurtosis is very strong. The realizations in the middle regime no longer seem to be uniformly distributed (upper right panel). They are distributed around zero with the highest probability instead because they are also inside the band attracted to a unique value which is the long-run equilibrium at zero.

\footnotetext{
${ }^{16}$ Parity is represented by the long-run equilibrium which is identical with zero in this graph.
} 

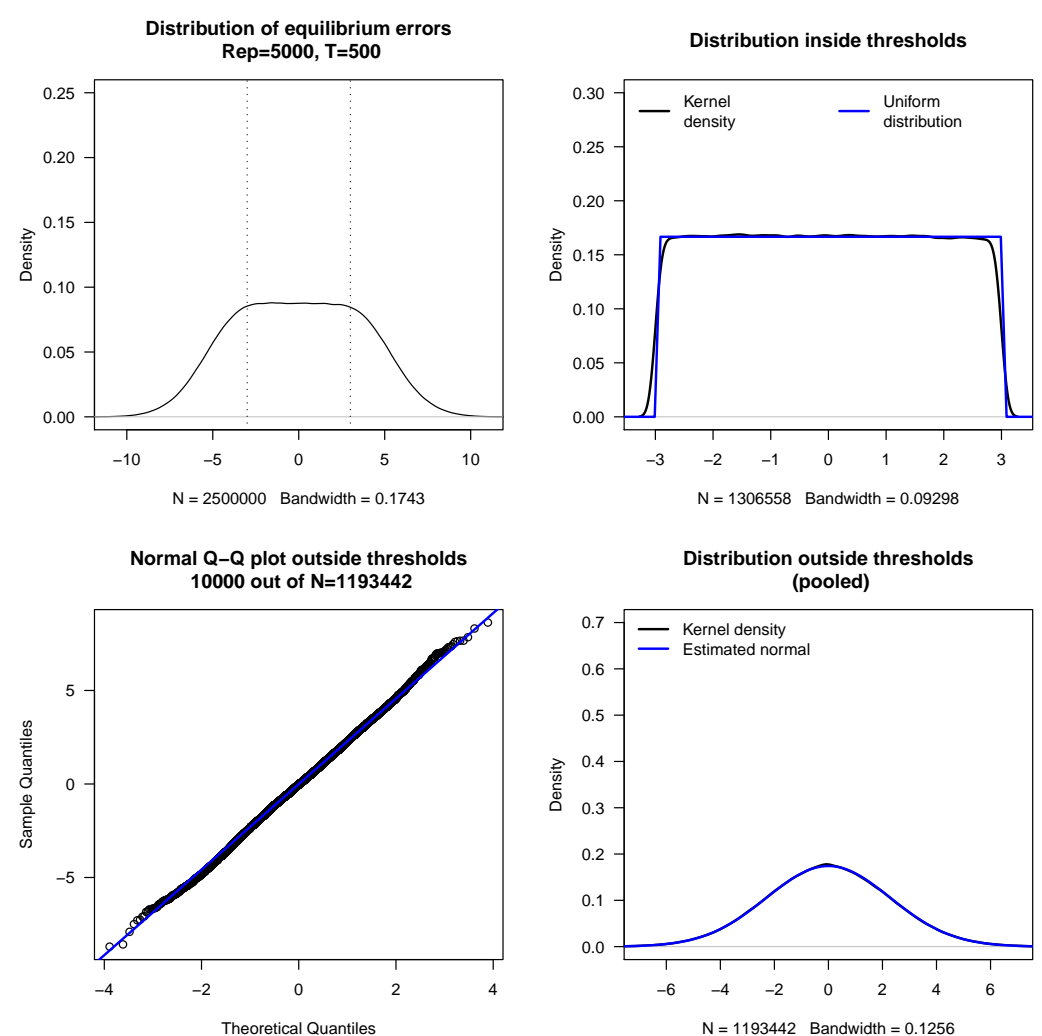

Note: The parameters are chosen according to the model in Balke and Fomby (1997, eq. (1) and (2)). The chosen parameters are: $\theta^{(1)}=-3, \theta^{(2)}=3, \epsilon_{t} \sim N I D(0,1), \eta_{t} \sim N I D(0,1), \rho^{(1)}=\rho^{(3)}=0.9$ and $\alpha=-2, \beta=1$. Hence, the model corresponds to a symmetric and continuous Band-TVECM of three regimes. It has no autoregressive dynamics. The resulting adjustment speeds are $\gamma_{1}^{(1)}=\gamma_{1}^{(3)}=-0.0333$ and $\gamma_{2}^{(1)}=\gamma_{2}^{(3)}=0.0333$. The dotted lines in the upper left panel are the symmetric thresholds.

Figure 3.4: Distribution of Band-TVECM Realizations (Weak Adjustment)

The MSVECM appears to be a mixture of distributions which are close to normal distributions as Figure 3.8 illustrates. Although the distributions in each regime seem very close to normal, the sum of both seems to deviate stronger from normal due to a higher kurtosis. The question is whether a similar relationship holds for the distributions in the MSVECM regimes as for the ones of the TVECM, that is, whether the distribution of the innovations dominates the regimes' distribution if its adjustment is weak. 

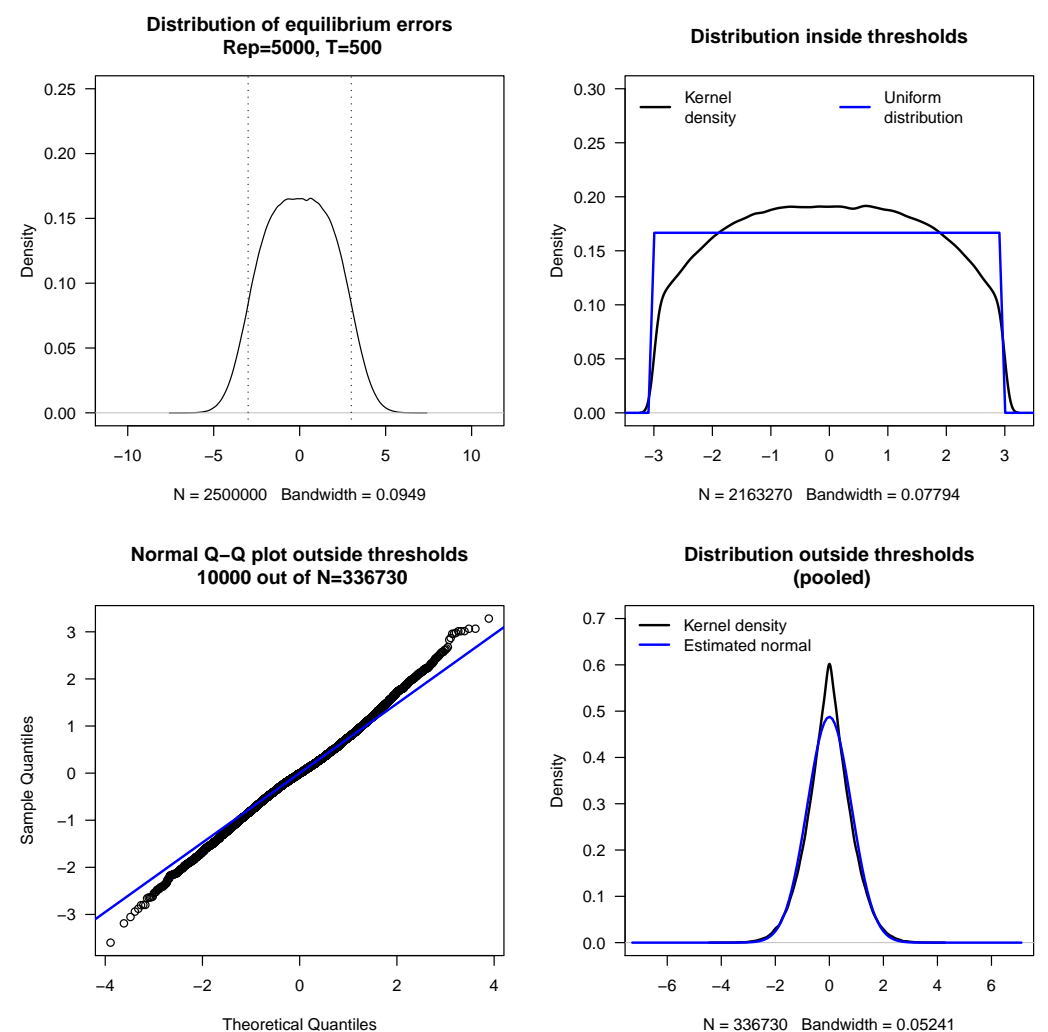

Note: The parameters are identical to the ones used in Figure 3.4 except $\rho^{(1)}=\rho^{(3)}=-0.95$ implying a symmetric and continuous Band-TVECM with $\gamma_{1}^{(1)}=\gamma_{1}^{(3)}=-0.65$ and $\gamma_{2}^{(1)}=\gamma_{2}^{(3)}=0.65$. The dotted lines in the upper left panel are the symmetric thresholds.

\section{Figure 3.5: Distribution of Band-TVECM Realizations (Strong Adjustment)}

Consequently, both model families imply similar distributions for some cases. An approximation of the unknown distributions by mixtures of normals seems appropriate in such cases. However, the distributions implied by some cointegration models depend on the nature of the underlying time series process, that is, its RGP and the strength of the error-correction. Therefore, the distributions implied by the model families can differ considerably for some settings. All simulations have been carried out for simple models without autoregressive dynamics. Hence, the simulated models correspond to the $\mathbf{R}$-form mentioned in Section 1.3.2. We conjecture that these dynamics also play a noticeable role in determining the resulting distributions of cointegration models and, hence, may lead to complicated distributions. Nevertheless, it is not certain whether the distributions assumed PBM context or implied by various versions of cointegration models are appropriate for observed price data. Hence, future research on the distributional characteristics of observed price data might shed some light on this question. 

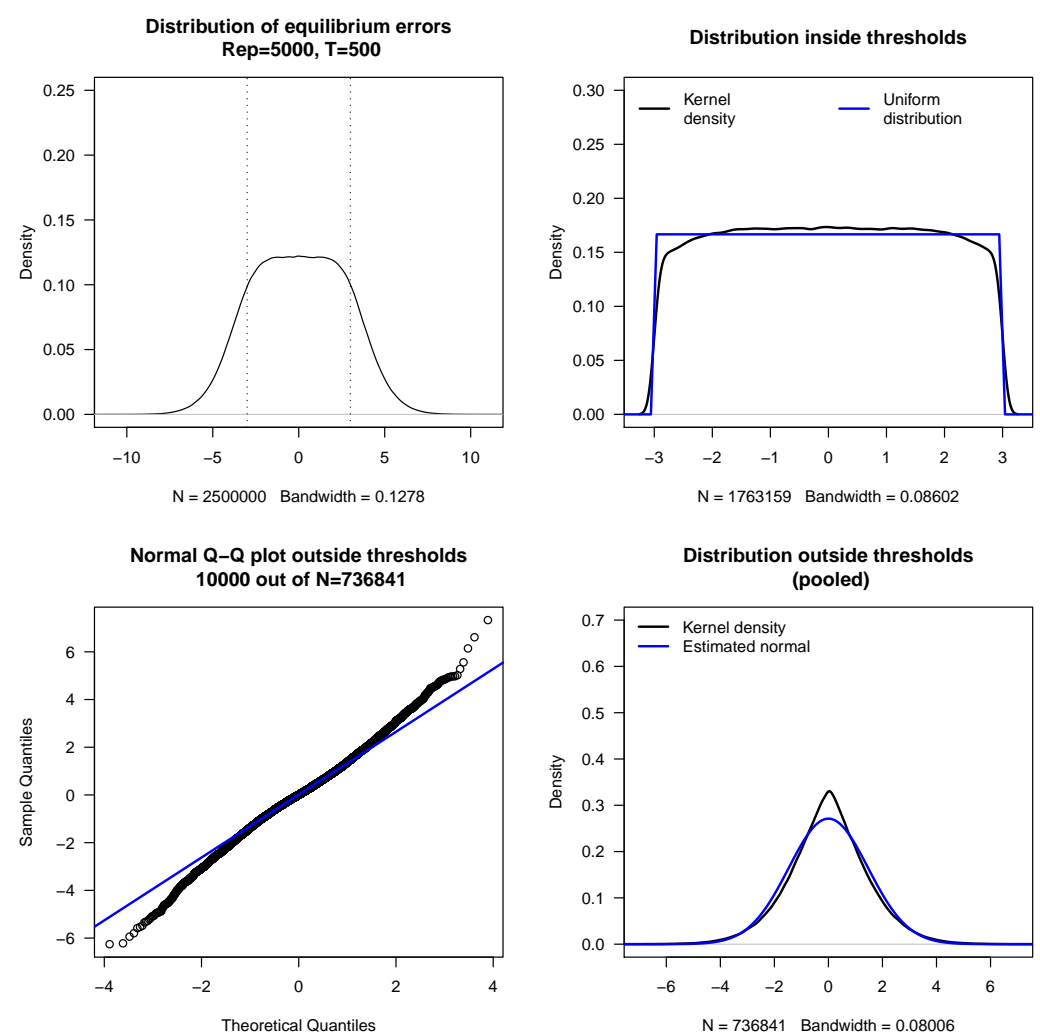

Note: The parameters are chosen according to the model in Balke and Fomby (1997, eq. (1) and (2)). The chosen parameters are: $\theta^{(1)}=-3, \theta^{(2)}=3, \epsilon_{t} \sim N I D(0,1), \eta_{t} \sim N I D(0,1), \rho^{(1)}=\rho^{(3)}=0.9, \rho^{(2)}=0$ and $\alpha=-2, \beta=1$. Hence, the model corresponds to a symmetric EQ-TVECM of three regimes. It has no autoregressive dynamics. The resulting adjustment speeds are $\gamma_{1}^{(1)}=\gamma_{1}^{(3)}=-0.0333$ and $\gamma_{2}^{(1)}=\gamma_{2}^{(3)}=0.0333$. The dotted lines in the upper left panel are the symmetric thresholds.

Figure 3.6: Distribution of EQ-TVECM Realizations (Weak Adjustment) 

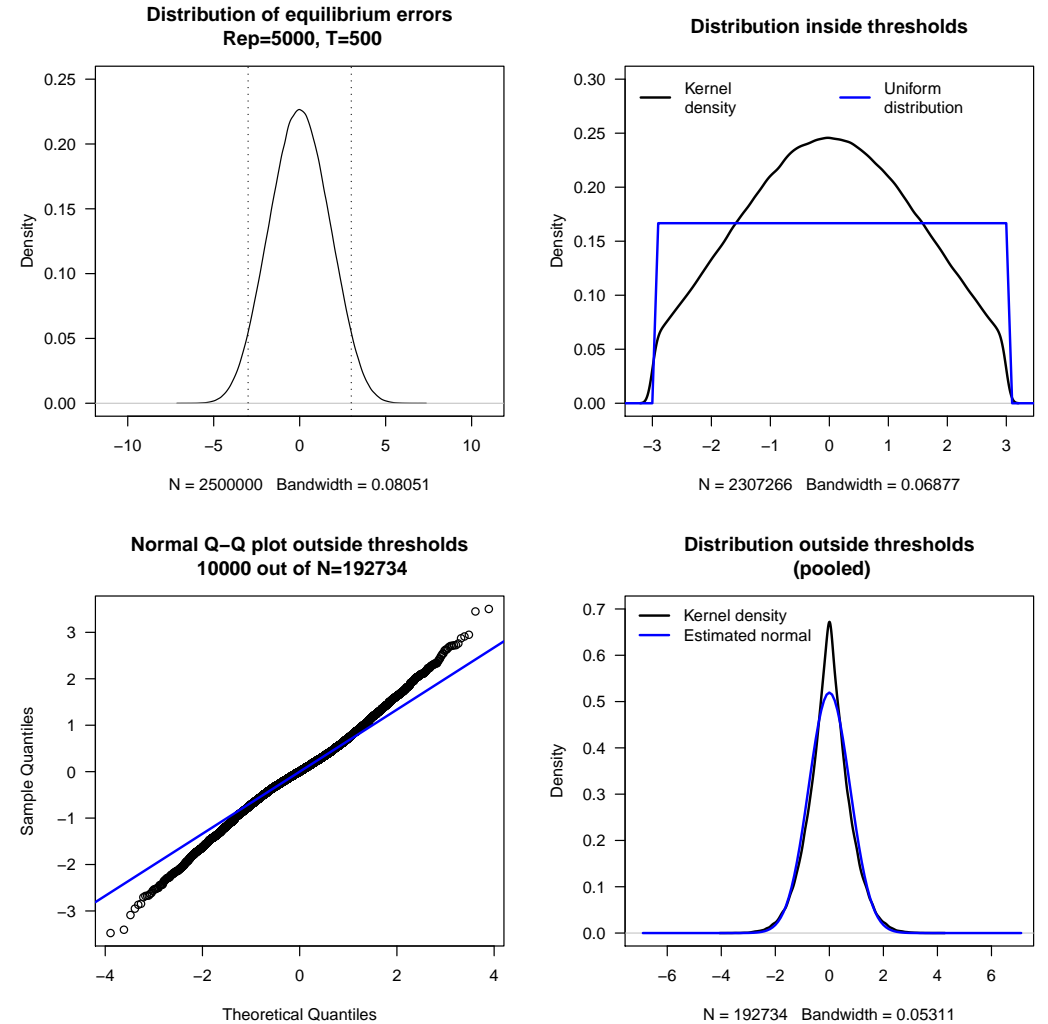

Note: The parameters are identical to the ones used in Figure 3.6 except for $\rho^{(1)}=\rho^{(3)}=0.1$ implying a symmetric EQ-TVECM with $\gamma_{1}^{(1)}=\gamma_{1}^{(3)}=-0.3, \gamma_{2}^{(1)}=\gamma_{2}^{(3)}=0.3$, and $\gamma_{1}^{(2)}=\gamma_{1}^{(2)}=0$. The dotted lines in the upper left panel are the symmetric thresholds.

Figure 3.7: Distribution of EQ-TVECM Realizations (Strong Adjustment) 

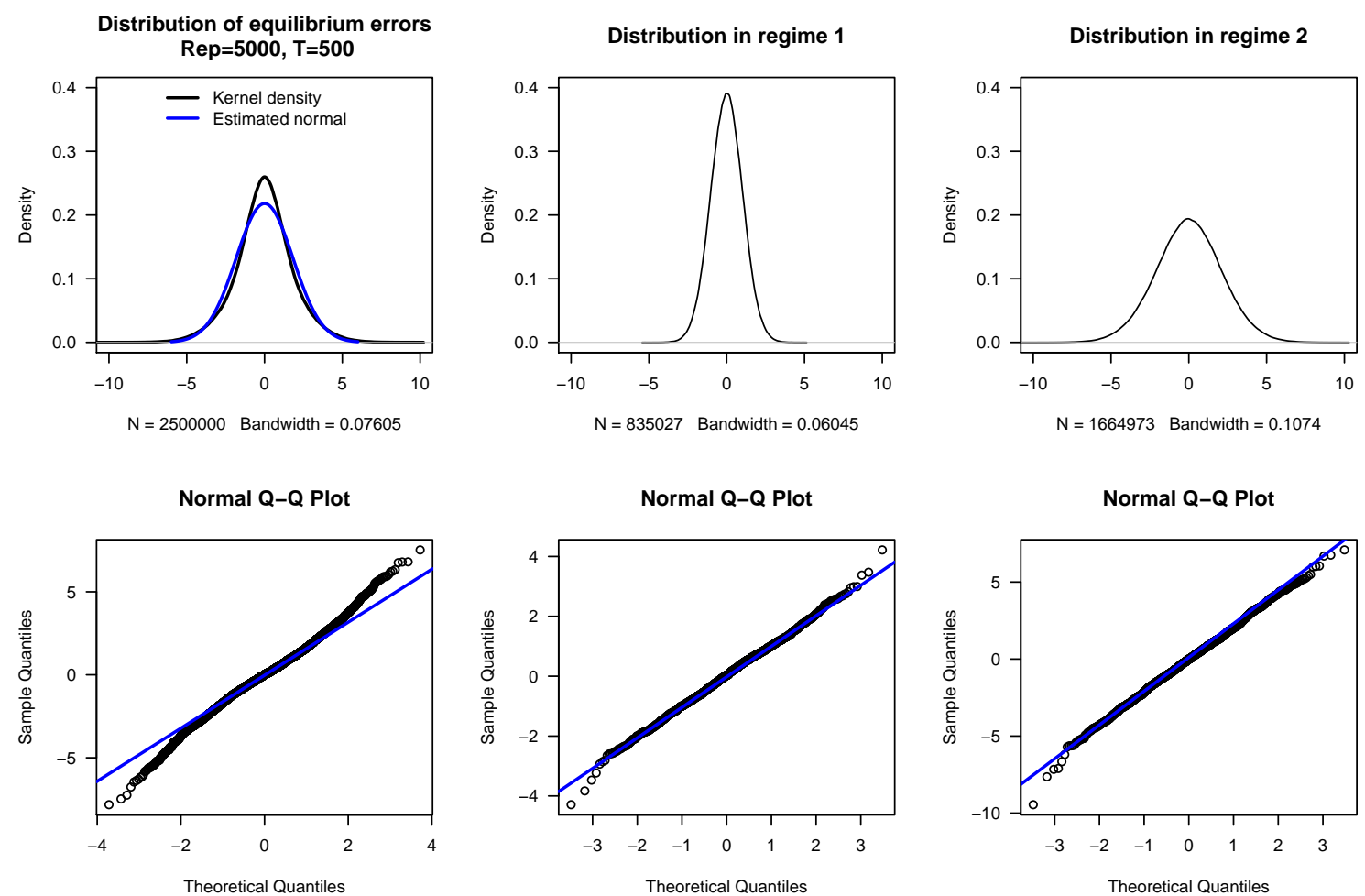

Note: The parameters are chosen according to Balke and Fomby (1997, eq. (1) and (2)) with the difference that $z_{t}$ follows a Markov-chain instead of a threshold process. The chosen parameters are identical to the ones used in Figure 2.7, p. 41 except for stronger error-correction in regime 1 of magnitude $\alpha^{(1)}=(-0.40 .4)^{\top}$.

Figure 3.8: Distribution of MSVECM Realizations (Strong vs. Weak Adjustment) 



\section{Conclusion}

Chapter 2 presents four model classes suitable for the analysis of nonlinear price transmission, that is, price transmission which is characterized by nonconstant adjustment towards equilibrium (error-correction). Three of these classes belong to the family of cointegration models, while the parity bounds model is a mixture distribution model. The cointegration approaches either model the nonlinearities by parametric piecewise linear functions (so-called regime-dependent models for which model parameters are constant, i.e., linear in each regime) or in a flexible nonparametric fashion. Regimedependent models assume a certain regime-generating process which characterizes each model. The researcher has to make a choice for one process before model specification and estimation. Cointegration approaches usually use, in contrast to the parity bounds model class, only price time series data. However, they provide estimates on the time series dynamics of the data, the stochastic model of the regime switching mechanism and the regime classification of the observations. The class of parity bounds models additionally relies on transaction cost and/or trade flow data and focuses on the results of dynamic processes by only classifying the observations into regimes.

Some of the presented model classes contain a large number of potential sub-models and specifications. However, all models of one class are characterized by a certain mechanism modeling nonlinearities in price transmission. Threshold time series models require the variable(s) inducing the regime switches to be observed. In the case of Markov-switching time series models, the determinants of the regime switching may remain unspecified. Currently, regime-dependent cointegration models characterized by discrete shifts in error-correction parameters are most frequently applied although models which imply a smooth parameter change are increasingly implemented. Since econometric techniques for testing nonlinear model classes against each other are very challenging to develop, detailed knowledge of the underlying mechanisms inducing the regime switching, as this thesis aims to provide, may facilitate model selection. Flexible model classes such as the semiparametric vector error-correction model may play an increasing role in future research, e.g., for the choice of the parametric approximation of nonlinearities. Each model class has its strengths and weaknesses. For the applied researcher, the question which remains to be answered is how well the particular properties of data are accounted for by the chosen model. Moreover, how restrictive are the assumptions underlying the chosen model with respect to the data at hand? From a pragmatic point of view, the (limited) data availability might play a role in model selection. Furthermore, some model classes promise interesting extensions so 
that they can be used to analyze novel aspects in price transmission analysis.

All of the discussed model classes are capable to provide a more adequate description of reality. Nonconstant parameters either in the form of regime-dependence or smooth changes may be caused by a number of (potentially interfering) impacts. From the empirical analysis point of view, such impacts can hardly be distinguished from each other. Similarly, the question concerning the dominant source of nonlinearities may be difficult to answer. One means which might provide evidence on these issues is careful analysis of the model's residuals. Besides the development of the underlying econometric theory in general, theoretical results are needed particularly with respect to estimation and the twofold problem of model selection in this context, which consists of the choice of a linear vs. a nonlinear model and the selection of the most adequate specification among several competing nonlinear models.

A general problem within this area of research pertains to the availability of reliable data. Sporadically, more than just price data is available. Data on transaction costs and trade flows exist often on a highly aggregated scale which is too "coarse" both in the space and time dimensions to be used in price transmission analysis. International organizations, such as the Worldbank or the FAO, have intensified their efforts in recent years in gathering disaggregated data particularly for staple foods in developing countries. In consequence, the available information for economic analysis and policy assessment has been growing considerably during the last two decades. Hence, prospects regarding this issue seem promising.

An important aspect of future research is the analysis of determinants of market integration and linear and nonlinear price transmission. Appendix C, pp. 139, provides an example of such an analysis. It assesses determinants of the transmission of maize prices within and in-between the three largest member states of the East African Community: Kenya, Tanzania, and Uganda. Besides econometrics, qualitative research based on questionnaires etc. might play an important role in this context in order to identify major determinants. ${ }^{1}$

Furthermore, the explicit consideration of the spatial structure of markets might provide a promising field for future research. Although price transmission analysis uses prices which are the result of economic interdependencies across space (determined by the particular spatial structure among other influences), such information is not explicitly used for analysis up to now. Given the general concern of data availability, this kind of data might represent a source of information which can relatively easily be obtained.

\footnotetext{
1 For example, Mittelhammer (2009) argues for a broader methodological toolbox to be employed in applied economics. Madi et al. (2009) or World Bank (2009) are examples of such qualitative research.
} 




\section{Bibliography}

Agra Europe (2008). "Grain prices hit records again." Agra Europe No. 2301: M/2$\mathrm{M} / 3$.

Agüero, J.M. (2007). "Asymmetric Price Adjustments and Behavior Under Risk: Evidence from Peruvian Agricultural Markets." Working paper, University of California, Riverside, USA.

Akerlof, G.A. (2009). "How Human Psychology Drives the Economic and Why it Matters." American Journal of Agricultural Economics 91(5): 1175.

Alemu, Z.G., and G.R. Biacuana (2006). "Measuring Market Integration in Mozambican Maize Markets: A Threshold Vector Error Correction Approach." Paper presented at the IAAE 2006 - The XXVI International Conference on Agricultural Economics, Gold Cost, Australia, August 12-18.

Anderson, T.W. (1951). "Estimating Linear Restrictions on Regression Coefficients for Multivariate Normal Distributions." Annals of Mathematical Statistics 22(3): 327-351.

Azariadis, C. (1981). "Self-Fulfilling Prophecies." Journal of Economic Theory 25: 380-396.

Baghli, M. (2005). "Nonlinear Error-Correction Models for the FF/DM Rate." Studies in Nonlinear Dynamics and Econometrics 9(1): Article 7.

Bai, J. (1997). "Estimating Multiple Breaks One at a Time." Econometric Theory 13: 315-352.

Bai, J., and P. Perron (1998). "Estimating and Testing Linear Models with Multiple Structural Changes." Econometrica 66: 47-78.

Bakucs, L.Z., and I. Fertö (2007). "Spatial Integration on the Hungarian Milk Market." Paper prepared for presentation at the Joint IAAE - 104th EAAE Seminar Agricultural Economics and Transition, Corvinus University, Budapest, Hungary, September 6-8.

Balcombe, K.G., and J. Morrison (2002). "Commodity Price Transmission: A Critical Review of Techniques and an Application to Selected Export Commodities." Report to the Food and Agriculture Organization of the United Nations, Rome, Italy.

Balcombe, K.G., and G. Rapsomanikis (2008). "Bayesian Estimation and Selection of Nonlinear Vector Error Correction Models: The Case of the Sugar-Ethanol-Oil Nexus in Brazil." American Journal of Agricultural Economics 90(3): 658-668. 
Balcombe, K.G., A. Bailey, and J. Brooks (2007). "Threshold Effects in Price Transmission: The Case of Brazilian Wheat, Maize, and Soya Prices." American Journal of Agricultural Economics 89(2): 308-323.

Balke, N.S., and T.B. Fomby (1997). "Threshold Cointegration." International Economic Review 38: 627-645.

Barrett, C.B. (1996). "Market Analysis Methods: Are Our Enriched Toolkits WellSuited to Enlivened Markets?" American Journal of Agricultural Economics 78: 825-829.

Barrett, C.B. (2001). "Measuring Integration and Efficiency in International Agricultural Markets." Review of Agricultural Economics 23: 19-32.

Barrett, C.B., and J.R. Li (2002). "Distinguishing Between Equilibrium and Integration in Spatial Price Analysis." American Journal of Agricultural Economics 84: 292-307.

Barrett, C.B., J.R. Li, and D. Bailey (2000). "Factor and Product Market Tradability and Equilibrium in Pacific Rim Pork Industries." Journal of Agricultural and Resource Economics 25(1): 68-87.

Baulch, R.J. (1994). "Spatial Price Equilibrium and Food Market Integration." Ph.D. Thesis, Food Research Institute, Stanford University, Stanford, USA.

Baulch, R.J. (1999). "Transfer Costs, Spatial Arbitrage, and Testing for Food Market Integration." American Journal of Agricultural Economics 79(2): 477-487.

Bec, F., and A. Rahbek (2004). "Vector Equilibrium Correction Models with Nonlinear Discontinuous Adjustments." Econometrics Journal 7: 628-651.

Bekkerman, A., B.K. Goodwin, and N. Piggott (2009). "Spatial Analysis of Market Linkages in North Carolina Using Threshold Autoregression Models with Variable Transaction Costs." Paper presented at the AAEA \& ACCI Joint Annual Meeting, Milwaukee, USA, 26-28 July.

Ben-Kaabia, M., and J.M. Gil (2007). "Asymmetric Price Transmission in the Spanish Lamb Sector." European Review of Agricultural Economics 34: 53-80.

Ben-Kaabia, M., J.M. Gil, and M. Ameur (2005). "Vertical Integration and Non-linear Price Adjustments: The Spanish Poultry Sector." Agribusiness 21: 253-271.

Ben-Kaabia, M., J.M. Gil, and L. Boshnjaku (2002). "Price Transmission Asymmetries in the Spanish Lamb Sector." Paper prepared for presentation at the Xth EAAE Congress 'Exploring Diversity in the European Agri-Food System', Zaragoza, Spain, August 28-31.

Bhansali, R.J. (1980). Discussion of Tong and Lim (1980) p. 270.

Bhar, R., and S. Hamori (2007). "Analysing Yield Spread and Output Dynamics in an Endogenous Markov Switching Regression Framework." Asia-Pacific Financial Markets 14(1): 141-156.

Bossaerts, P. (1988). "Common Nonstationary Components of Asset Prices." Journal of Economic Dynamics and Control 12: 347-364. 
Box, G.E.P., and N.R. Draper (1987). Empirical Model-Building and Response Surfaces. Wiley, New York, USA.

Brümmer, B., S. von Cramon-Taubadel, and S. Zorya (2009). "The Impact of Market and Policy Instability on Price Transmission between Wheat and Flour in Ukraine." European Review of Agricultural Economics 36(2): 203-230.

Brümmer, B., S. von Cramon-Taubadel, R. Ihle, and J.-P. Loy (2004). "Spatial Effects in Regional Market Integration for Wheat in Ukraine." Paper presented at IAMO Forum 2004, Halle (Saale), Germany, November 4-6.

Busse, S., and B. Brümmer (2009a). "Bestimmung der Determinanten der Rapspreisentwicklung in der Hochpreisphase auf Basis von Markovzeitreihenmodellen." Paper presented at the 49. Jahrestagung der GeWiSoLa, Christian-Albrechts-Universität zu Kiel, Germany, September 30-October 2.

Busse, S., and B. Brümmer (2009b). "Price Formation in the German Diesel-BiodieselRapeseed-Soy Oil System: A Markov-switching VECM Approach." Paper presented at IAMO Forum 2009, Halle, Germany, June 17-19.

Busse, S., and R. Ihle (2009). "German Rapeseed Oil and Biodiesel Pricing under Changing Market Conditions: A Markov-switching Vector Error Correction Model Approach." Paper presented at IAAE 2009 - The XXVII International Conference on Agricultural Economics, Beijing, China, August 16-22.

Camacho, M. (2005). "Markov-Switching Stochastic Trends and Economic Fluctuations." Journal of Economic Dynamics and Control 29: 135-158.

van Campenhout, B. (2007). "Modelling Trends in Food Market Integration: Method and an Application to Tanzanian Maize Markets." Food Policy 32: 112-127.

Cass, D., and K. Shell (1983). "Do Sunspots Matter?" Journal of Political Economy 91: 193-227.

Chamley, C. (1999). "Coordinating Regime Switches." The Quarterly Journal of Economics 114: 869-905.

Chan, K.S. (1993). "Consistency and Limiting Distribution of the Least Squares Estimator of a Threshold Autoregressive Model." The Annals of Statistics 21: 520-533.

Chen, L.-H., M. Finney, and K.S. Lai (2005). "A Threshold Cointegration Analysis of Asymmetric Price Transmission from Crude Oil to Gasoline Prices." Economics Letters 89: 233-239.

Chung, K.L. (1960). Markov Chains with Stationary Transition Probabilities. SpringerVerlag, Berlin, Germany.

Cirera, A., and A. Channing (2008). "Measuring the Impact of Road Rehabilitation on Spatial Market Efficiency in Maize Markets in Mozambique." Agricultural Economics 39: $17-28$.

Clements, M.P., and H.-M. Krolzig (1998). "A Comparison of the Forecast Performance of Markov-Switching and Threshold Autoregressive Models for US GDP." Econometrics Journal 1: C47-C75. 
Coleman, A.M.G. (1995). "Arbitrage, Storage and the 'Law of One Price': New Theory for the Time Series Analysis of an Old Problem." Working paper, Princeton University, Princeton, USA.

Coleman, A.M.G. (2004). "Storage, Slow Transport, and the Law of One Price: Evidence from the Nineteenth Century U.S. Corn Market." Discussion Paper No. 502, University of Michigan, Ann Arbor, USA.

Cologni, A., and M. Manera (2009). "The Asymmetric Effects of Oil Shocks on Output Growth: A Markov-switching Analysis for the G-7 Countries." Economic Modelling 26(1): 1-29.

Conforti, P. (2004). "Price Transmission in Selected Agricultural Markets." FAO Commodity and Trade Policy Research Working Paper No. 7, Rome, Italy, March.

von Cramon-Taubadel, S. (1998). "Estimating Asymmetric Price Transmission with the Error Correction Representation: An Application to the German Pork Market." European Review of Agricultural Economics 25: 1-18.

von Cramon-Taubadel, S., and S. Fahlbusch (1994). "Identifying Asymmetric Price Transmission with Error Correction Models." Poster presented at EAAE European Seminar, Reading, UK, September 19-21.

von Cramon-Taubadel, S., and J.-P. Loy (1996). "Price Asymmetry in the International Wheat Market: Comment." Canadian Journal of Agricultural Economics 44: 311317.

Davidson, R., and R. MacKinnon (2004). Econometric Theory and Methods. Oxford University Press, New York, USA.

Dempster, A.P., N.M. Laird, and D.B. Rubin (1977). "Maximum Likelihood from Incomplete Data via the EM Algorithm (with Discussion)." Journal of the Royal Statistical Society B 39: 1-38.

Dercon, S., and B. van Campenhout (1998). "Dynamic Price Adjustment in Spatially Separated Food Markets with Transaction Costs." Working paper, Katholieke Universiteit Leuven, Belgium.

Diebold, F.X., J.-H. Lee, and G. Weinbach (1994). "Regime Switching with TimeVarying Transition Probabilities." In C.P. Hargreaves (ed): Nonstationary Time Series Analysis and Cointegration, Oxford University Press, Oxford, UK, chapter 10.

van Dijk, D., T. Teräsvirta, and P.H. Franses (2002). "Smooth Transition Autoregressive Models - A Survey of Recent Developments." Econometric Reviews 21(1): $1-47$.

Doornik, J.A. (2002). Object-Oriented Matrix Programming Using Ox, Timberlake Consultants Press, London, UK. Available at: http://www.doornik.com/index.html.

Dorsey, R.E., and W.J. Mayer (1995). "Genetic Algorithms for Estimation Problems with Multiple Optima, Nondifferentiability, and Other Irregular Features." Journal of Business and Economic Statistics 13: 53-66. 
Dumas, B. (1992). "Dynamics Equilibrium and the Real Exchange Rate in a Spatially Separated World." Review of Financial Studies 5: 153-180.

Ehrmann, M., M. Ellison, and N. Valla (2001). "Regime-dependent Impulse Response Functions in a Markov-switching Vector Autoregression Model." Bank of Finland Discussion Papers 11, Helsinki, Finland.

Ejrnæs, M., and K.G. Persson (2001). "Market Integration and Transport Costs in France 1825-1903: A Threshold Error Correction Approach to the Law of One Price." Explorations in Economic History 37: 149-173.

Enders, W. (2004). Applied Econometric Time Series. John Wiley and Sons, Hoboken, USA.

Engle, R.F., and C.W.J. Granger (1987). "Co-Integration and Error Correction: Representation, Estimation and Testing." Econometrica 55: 251-276.

Enke, S. (1951). "Equilibrium among Spatially Separated Markets: Solution by Electrical Analogue." Econometrica 19: 40-47.

Escobal, J. (2005). "The Role of Public Infrastructure in Market Development in Rural Peru." Ph.D. Thesis, Wageningen University, The Netherlands.

Escribano, A. (1985). "Nonlinear Error-correction: The Case of Money Demand in the U.K. (1878-1970)." Working paper, Department of Economics, University of California, San Diego, USA.

Escribano, A. (1986). "Identification and Modelling of Economic Relationships in a Growing Economy." Ph.D. Thesis, Department of Economics, University of California, San Diego, USA, chapter 4.

Escribano, A. (2004). "Nonlinear Error Correction: The Case of Money Demand in the United Kingdom (1878-2000)." Macroeconomic Dynamics 8: 76-116.

Escribano, A., and S. Mira (2002). "Nonlinear Error Correction Models." Journal of Time Series Analysis 23: 509-522.

Escribano, A., and G. Pfann (1998). "Nonlinear Error Correction, Asymmetric Adjustment and Cointegration." Economic Modelling 15: 197-216.

Fackler, P.L., and B.K. Goodwin (2001). "Spatial Price Analysis." In B. Gardner, and G. Rausser (eds): Handbook of Agricultural Economics, Vol. 1, Elsevier, Amsterdam, The Netherlands, pp. 971-1024.

Fackler, P.L., and H. Tastan (2008). "Estimating the Degree of Market Integration." American Journal of Agricultural Economics 90(1): 69-85.

Fanizza, D.G. (1990). "Multiple Steady States and Coordination Failures in Search Equilibrium: New Approaches to the Business Cycle." Ph.D. Thesis, Northwestern University, Evanston, USA.

Federico, G. (2007). "Market Integration and Market Efficiency: The Case of 19th Century Italy." Explorations in Economic History 44: 293-316. 
Francis, N., and M. Owyang (2003). "Asymmetric Common Trends: An Application of Monetary Policy in a Markov-Switching VECM." Federal Reserve Bank of St. Louis Working Paper 2003-001B, St. Louis, USA.

Frei, L. (2008). "The Markov-switching Vector Error Correction Model - Dynamics, Bayesian Inference, and Application to the Spot and Forward Swiss Franc - US dollar Exchange Rates." Ph.D. Thesis, University of Basel, Switzerland.

Frey, G., and M. Manera (2007). "Econometric Models of Asymmetric Price Transmission." Journal of Economic Surveys 21(2): 349-415.

Frisch, R., and F. Waugh (1933). "Partial Time Regressions as Compared with Individual Trends." Econometrica 45: 939-953.

Garratt, A., K. Lee, M.H. Pesaram, and Y. Shin (2006). Global and National Macroeconomic Modelling: A Long-Run Structural Approach. Oxford University Press, Oxford, UK.

Gaul, J. (2008). "Three Essays on Unit Roots and Nonlinear Co-Integrated Processes." Ph.D. Thesis, Rheinische Friedrich-Wilhelms-Universität Bonn, Germany.

Gaul, J., and E. Theissen (2008). "A Partially Linear Approach to Modelling the Dynamics of Spot and Futures Prices." Working paper, Rheinische Friedrich-WilhelmsUniversität Bonn, Germany.

Goldfeld, S.M., and R.E. Quandt (1973). "A Markov Model for Switching Regressions." Journal of Econometrics 1: 3-16.

Gonzalez-Rivera, G., and S.M. Helfand (2001). "The Extent, Pattern, and Degree of Market Integration: A Multivariate Approach for the Brazilian Rice Market." American Journal of Agricultural Economics 83: 576-592.

Gonzalo, J. (1994). "Five Alternative Methods of Estimating Long-run Equilibrium Relationships." Journal of Econometrics 60: 203-233.

Gonzalo, J., and J.V. Pitarakis (2006). "Threshold Effects in Cointegrating Relationships." Oxford Bulletin of Economics and Statistics 68: 813-833.

Goodwin, B.K., and T.J. Grennes (1998). "Tsarist Russia and the World Wheat Market." Explorations in Economic History 35: 405-430.

Goodwin, B.K., and D.C. Harper (2001). "Price Transmission, Threshold Behavior, and Asymmetric Adjustment in the U.S. Pork Sector." Journal of Agricultural and Applied Economics 32: 543-553.

Goodwin, B.K., and M.T. Holt (1999). "Price Transmission and Asymmetric Adjustment in the U.S. Beef Sector." American Journal of Agricultural Economics 81: 630-637.

Goodwin, B.K., and N. Piggott (2001). "Spatial Market Integration in the Presence of Threshold Effects." American Journal of Agricultural Economics 83: 302-317.

Goodwin, B.K., and P. Vavra (2009). "What Can We Learn from Spatial and Vertical Price Transmission Studies? Empirical Examples from U.S. Meat Markets." Paper prepared for the Courant Research Centre "Poverty, Equity and Growth" Inaugural Conference at the University of Göttingen, Germany, July 1-3. 
Goodwin, B.K., T.J. Grennes, and L.A. Craig (2002). "Mechanical Refrigeration and the Integration of Perishable Commodity Markets." Explorations in Economic History 39: 154-182.

Granger, C.W.J., and T.H. Lee (1989). "Investigation of Production, Sales and Inventory Relationships Using Multicointegration and Non-symmetric Error-correction Models." Journal of Applied Econometrics 4: S145-S159.

Granger, C.W.J., and T. Teräsvirta (1993). Modelling Nonlinear Economic Relationships. Oxford University Press, New York, USA.

Hall, S., Z. Psaradakis, and M. Sola (1997). "Switching Error-Correction Models of House Prices in the United Kingdom." Economic Modelling 14: 517-527.

Hamilton, J.D. (1989). "A New Approach to the Economic Analysis of Nonstationary Time Series and the Business Cycle." Econometrica 57: 357-384.

Hamilton, J.D. (1990). "Analysis of Time Series Subject to Regime Changes." Journal of Econometrics 45: 39-70.

Hamilton, J.D. (1994). Time Series Analysis. Princeton University Press, Princeton, USA.

Hamilton, J.D. (1995). "Rational Expectations and the Economic Consequences of Changes in Regime." In K.D. Hoover (ed): Macroeconometrics: Developments, Tensions, and Prospects, Kluwer Academic Publishers, Boston, USA, chapter 9.

Hamilton, J.D. (1996). "Specification Testing in Markov-switching Time Series Models." Journal of Econometrics 70: 127-157.

Hamilton, J.D., and B. Raj (2002a, eds). Advances in Markov-switching Models. Applications in Business Cycle Research and Finance. Physica-Verlag, Heidelberg, Germany.

Hamilton, J.D., and B. Raj (2002b). "New Directions in Business Cycle Research and Financial Analysis." In Hamilton, J.D., and B. Raj (eds): Advances in Markovswitching Models. Applications in Business Cycle Research and Finance, PhysicaVerlag, Heidelberg, Germany, chapter 1.

Hansen, B.E. (1992). "Erratum: The Likelihood Ratio Test under Nonstandard Conditions: Testing the Markov Switching Model of GNP." Journal of Applied Econometrics 11, 195-198.

Hansen, B.E. (1997). "Inference in TAR Models." Studies in Nonlinear Dynamics and Econometrics 2: 1-14.

Hansen, B.E. (1999). "Testing for Linearity." Journal of Economic Surveys 13: 551576.

Hansen, B.E. (2001). "Sample Splitting and Threshold Estimation." Econometrica 68: 575-603.

Hansen, B., and P.C.B. Phillips (1990). "Estimation and Inference in Models of Cointegration: A Simulation Study." Advances in Econometrics 8: 225-248. 
Hansen, B.E., and B. Seo (2002). "Testing for Two-Regime Threshold Cointegration in Vector Error-Correction Models." Journal of Econometrics 110: 293-318.

Härdle, W., M. Müller, S. Sperlich, and A. Werwatz (2004). Nonparametric and Semiparametric Models. Springer-Verlag, Heidelberg, Germany.

Hassouneh, I., T. Serra, and J.M. Gil (2010). "Price Transmission in the Spanish Bovine Sector: the BSE Effect." Agricultural Economics 41: 33-42.

Heckscher, E.F. (1916). "Växelkurens Grundval vid Pappersmynfot." Economisk Tidskrift 18: 309-312.

Hendry, D.F., and K. Juselius (2001). "Explaining Cointegration Analysis: Part I." The Energy Journal 21: 1-42.

Hendry, D.F., and K. Juselius (2001). "Explaining Cointegration Analysis: Part II." The Energy Journal 22: 75-120.

Howitt, P., and R.P. McAfee (1992). "Animal spirits." The American Economic Review 82: $493-507$.

Ihle, R., J. Amikuzuno, and S. von Cramon-Taubadel (2010). "The Integration of Tomato Markets in Ghana With and Without Direct Trade Flows." Revue D'Économie du Développement (in press).

Jackman, S. (1995). "Re-Thinking Equilibrium Presidential Approval - Markovswitching Error Correction." Paper presented at the 12th Annual Political Methodology Summer Conference, Indiana University, Bloomington, USA, July 27-30.

Jacks, D.S. (2005). "Intra- and International Commodity Market Integration in the Atlantic Economy, 1800-1913." Explorations in Economic History 42: 381-413.

Jacks, D.S. (2006). "What Drove 19th Century Commodity Market Integration?" Explorations in Economic History 43: 383-412.

Jeanne, O., and P. Masson (2000). "Currency Crises, Sunspots and Markov-switching Regimes." Journal of International Economics 50: 327-350.

Jerzmanowski, M. (2006). "Empirics of Hills, Plateaus, Mountains and Plains - A Markov-switching Approach to Growth." Journal of Development Economics 81(2): 357-385.

Johansen, S. (1988). "Statistical Analysis of Cointegration Vectors." Journal of Economic Dynamics and Control 12: 231-254.

Johansen, S. (1991). "Estimation and Hypothesis Testing of Cointegration Vectors in Gaussian Vector Autoregressive Models." Econometrica 59(6): 1551-1580.

Johansen, S. (1995). Likelihood-Based Inference in Cointegrated Vector Autoregressive Models. Oxford University Press, Oxford, UK.

Johansen, S. (2005). "Interpretation of Cointegrating Coefficients in the Cointegrated Vector Autoregressive Model." Oxford Bulletin of Economics and Statistics 67(1): 93-104. 
Juselius, K. (2008). The Cointegrated VAR Model. Methodology and Applications. Oxford University Press, Oxford, UK.

Karlsen, H.A., T. Myklebust, and D. Tøstheim (2007). "Nonparametric Estimation in a Nonlinear Cointegration Type Model." The Annals of Statistics 351: 252-299.

Keynes, J.M. (1936). The General Theory of Employment, Interest, and Money. Macmillan, London, UK.

Kim, C.-J. (1994). "Dynamic Linear Models with Markov-switching." Journal of Econometrics 60: 1-22.

Kim, C.-J. (2004). "Markov-switching Models with Endogenous Explanatory Variables." Journal of Econometrics 122: 127-136.

Kim, C.-J. (2009). "Markov-switching Models with Endogenous Explanatory Variables II - A Two-step MLE Procedure." Journal of Econometrics 148: 46-55.

Klasra, M.A. (2009). "Cointegration, Causality and the Transmission of Shocks across Wheat Market in Pakistan." Quality and Quantity 43: 305-315.

Koop, G., M.H. Pesaran, and S.M. Potter (1996). "Impulse Response Analysis in Nonlinear Multivariate Models." Journal of Econometrics 74: 119-147.

Kourtellos, A., T. Stengos, and C.M. Tan (2009). "Structural Threshold Regression." Unpublished manuscript. Available at: SSRN: http://ssrn.com/abstract=1105931.

Kristensen, D., and A. Rahbek (2007). "Likelihood-Based Inference for Cointegration with Nonlinear Error-Correction." CREATES Research Paper 2007-38, School of Economics and Management, University of Aarhus, Denmark.

Krolzig, H.-M. (1996). "Statistical Analysis of Cointegrated VAR Processes with Markovian Regime Shifts." SFB 373 Discussion Paper 25/1996, HumboldtUniversität zu Berlin, Berlin, Germany.

Krolzig, H.-M. (1997). Markov-Switching Vector Autoregressions. Modelling, Statistical Inference, and Applications to Business Cycle Analysis. Springer-Verlag, Berlin, Germany.

Krolzig, H.-M. (2004). MSVAR - An Ox Package Designed for the Econometric Modelling of Univariate and Multiple Time Series Subject to Shifts in Regime, Version 1.31k. Available at: http://www.economics.ox.ac.uk/research/hendry/krolzig/msvar.html.

Krolzig, H.-M., and J. Toro (2001). "A New Approach to the Analysis of Business Cycle Transitions in a Model of Output and Employment." Department of Economics Discussion Paper Series No. 59, University of Oxford, Oxford, UK.

Krolzig, H.-M., M. Marcellino, and G. Mizon (2002). "A Markov-switching Vector Equilibrium Correction Model of the UK Labor Market." Empirical Economics 27: 233-254.

Lee, K.C., and M.H. Pesaran (1993). "Persistence Profiles and Business Cycle Fluctuations in a Disaggregated Model of UK Output Growth." Richerche Economiche 47: 293-322. 
Lo, M.C., and E. Zivot (2001). "Threshold Cointegration and Nonlinear Adjustment to the Law of One Price." Macroeconomic Dynamics 5: 533-576.

Lovell, M. (1963). "Seasonal Adjustment of Economic Time Series." Journal of the American Statistical Association 58: 993-1010.

Luoma, A., J. Luoto, and M. Taipale (2004). "Threshold Cointegration and Asymmetric Price Transmission in Finnish Beef and Pork Markets." Pellervo Economic Research Institute Working Papers No. 70, Helsinki, Finland.

Lutz, C., W.E. Kuiper, and A. van Tilburg (2006). "Maize Market Liberalisation in Benin: A Case of Hysteresis." Journal of African Economies 16: 102-133.

Lütkepohl, H. (1994). "Interpretation of Cointegrating Relations." Econometric Reviews 13: 391-394.

Lütkepohl, H. (2007). New Introduction to Multiple Time Series Analysis. SpringerVerlag, Berlin, Germany.

Lütkepohl, H., and M. Krätzig (2004, eds) . Applied Time Series Econometrics. Cambridge University Press, New York, USA.

MacDonald, I.L., and W. Zucchini (1997). Hidden Markov and Other Models for Discrete-valued Time Series. Chapman and Hall, London, UK.

Madi, A., H.A. Hassan, N. Al-Ghool, and O. Abu Gosh (2009). "The Impact of Closure and High Food Prices on Performance of Imported Staple Foods and Vegetable and Fruits Market in the oPt." Al-Sahel Co. for Institutional Development and Communications, report for the United Nations World Food Programme, Rome, Italy.

Mainardi, S. (2001). "Limited Arbitrage in International Wheat Markets: Threshold and Smooth Transition Cointegration." Australian Journal of Agricultural and Resource Economics 45(3): 335-360.

Mancuso, A.J., B.K. Goodwin, and T.J. Grennes (2003). "Nonlinear Aspects of Capital Market Integration and Real Interest Rate Equalization." International Review of Economics and Finance 12: 283-303.

Marshall A. (1890). Principles of Economics. Macmillan Company, New York, USA.

Martinez Peria, M.S. (2002). "A Regime-Switching Approach to the Study of Speculative Attacks: A Focus on EMS Crises." In J.D. Hamilton, and B. Raj (eds): Advances in Markov-Switching Models. Applications in Business Cycle Research and Finance, Physica-Verlag, Heidelberg, Germany, pp. 159-194.

McNew, K.P. (1996). "Spatial Market Integration: Definition, Theory, and Evidence." Agricultural and Resource Economics Review 25(1): 1-18.

McNew, K.P., and P.L. Fackler (1997). "Testing Market Equilibrium: Is Cointegration Informative?" Journal of Agricultural and Resource Economics 22: 191-207.

Mellows, M. (1999). Testen und Auswählen von nichtlinearen Zeitreihenmodellen mit dem Bootstrap-Verfahren. Peter Lang Europäischer Verlag der Wissenschaften, Frankfurt am Main, Germany. 
Meyer, J. (2004). "Measuring Market Integration in the Presence of Transaction Costs - a Threshold Vector Error Correction Approach." Agricultural Economics 31: 327334.

Meyer, J., and S. von Cramon-Taubadel (2004). "Asymmetric Price Transmission: A Survey." Agricultural Economics 50: 581-611.

Miljkovic, D. (1999). "The Law of One Price in International Trade: A Critical Review." Review of Agricultural Economics 21(1): 126-139.

Miljkovic, D., and R.J. Paul (2001). "Product Integration, Market Integration, and Relationships between Prices: An Application to World Salmon Markets: Comment." American Journal of Agricultural Economics 83(4): 1087-1089.

Mittelhammer, R. (2009). "Applied Economics-Without Apology." American Journal of Agricultural Economics 91(5): 1161-1174.

Mizrach, B., and J. Watkins (2000). "A Markov Switching Cookbook." In P. Rothman (ed): Nonlinear Time Series Analysis of Economic and Financial Data, Kluwer Academic Publishers, Boston, USA, chapter 2.

de Morais, I.A.C., and M.S. Portugal (2004). "Structural Change in the Brazilian Demand for Imports: A Regime Switching Approach." Paper presented at the Econometric Society 2004 Latin American Meeting, Santiago de Chile, Chile.

Moschini, G., and K.D. Meilke (1989). "Modelling the Pattern of Structural Change in U.S. Meat Demand." American Journal of Agricultural Economics 71: 253-261.

Moser, C., C. Barrett, and B. Minten (2009). "Spatial Integration at Multiple Scales: Rice Markets in Madagascar." Agricultural Economics 40: 281-294.

Negassa, A., and R.J. Myers (2007). "Estimating Policy Effects on Spatial Market Efficiency: An Extension to the Parity Bounds Model." American Journal of Agricultural Economics 89: 338-352.

Negassa, A., R. Myers, and E. Gabre-Madhin (2003). "Analyzing Grain Market Efficiency in Developing Countries: Review of Existing Methods and Extensions to the Parity Bounds Model." Markets, Trade and Institutions Division Discussion Paper No. 63, International Food Policy Research Institute, Washington D.C., USA.

Negassa, A., R. Myers, and E. Gabre-Madhin (2004). "Grain Marketing Policy Changes and Spatial Efficiency of Maize and Wheat Markets in Ethiopia." Markets, Trade and Institutions Division Discussion Paper No. 66, International Food Policy Research Institute, Washington D.C., USA.

Nesmith, T.D., and B.E. Jones (2008). "Linear Cointegration of Nonlinear Time Series with an Application to Interest Rate Dynamics." Studies in Nonlinear Dynamics and Econometrics 12(1): Article 6.

Noack, T. (2003). Probleme der SETAR-Modellierung in der Zeitreihenanalyse. Logos Verlag, Berlin, Germany.

Norman, S. (2007). "How Well does Nonlinear Mean Reversion Solve the PPP Puzzle?" Working Paper, University of Washington, Tacoma, USA. 
Obstfeld, M., and A.M. Taylor (1997). "Nonlinear Aspects of Goods-Market Arbitrage and Adjustment: Heckscher's Commodity Points Revisited." Journal of the Japanese and International Economies 11: 441-479.

O'Connel, P.G.J., and S.-J. Wei (1997). "The Bigger They Are, the Harder They Fall: How Price Differences across U.S. Cities are Arbitraged." Working paper 6089, National Bureau of Economic Research, Cambridge, USA.

O'Connel, P.G.J., and S.-J. Wei (2002). "'The Bigger They Are, the Harder They Fall': Retail Price Differences across U.S. Cities." Journal of International Economics 56: 21-53.

Owyang, M.T., J.M. Piger, H.J. Wall, and C.H. Wheeler (2007). "The Economic Performance of Cities: A Markov-switching Approach." Working paper 2006-056C, Federal Reserve Bank of St. Louis, USA.

Padilla-Bernal, L., D.D. Thilmany, and M.L. Loureiro (2003). "An Empirical Analysis of Market Integration and Efficiency for U.S. Fresh Tomato Markets." Journal of Agricultural and Resource Economics 28(3): 435-450.

Park, H., J.W. Mjelde, and D.A. Bessler (2007). "Time-Varying Threshold Cointegration and the Law of One Price." Applied Economics 39: 1091-1105.

Park, A., H. Jin, S. Rozelle, and J. Huang, (2002). "Market Emergence and Transition: Arbitrage, Transaction Costs, and Autarky in China's Grain Markets." American Journal of Agricultural Economics 84: 67-82.

Pede, V.O., and A.M. McKenzie (2005). "Integration in Benin Maize Market: An Application of Threshold Cointegration Analysis." Selected Paper prepared for presentation at the AAEA Annual Meeting, Providence, USA, July 24-27.

Pelagatti, M.M. (2005). "Time Series Modeling with Duration Dependent Markovswitching Vector Autoregressions - MCMC Inference, Software and Applications." Working paper, Department of Statistics, Università degli Studi di Milano-Bicocca, Italy.

Pelagatti, M.M. (2008). "Duration Dependent Markov-switching Vector Autoregression Properties, Bayesian Inference and Application to the Analysis of the US Business Cycle." In T. Nagakawa (ed): Business Fluctuations and Cycles, Nova Science Publishers, New York, pp. 43-66.

Phillips, P.C.B. (1991). "Spectral Regression for Cointegrated Time Series." In W.A. Barnett, J. Powell, and G. Tauchen (eds): Nonparametric and Semiparametric Methods in Econometrics and Statistics, Cambridge University Press, Cambridge, USA, pp. 413-435.

Potter, S.M. (1995). "A Nonlinear Approach to U.S. GNP." Journal of Applied Economics 10: 109-125.

Prakash, G., and A.M. Taylor (1997). "Measuring Market Integration: A Model of Arbitrage with an Econonometric Application to the Gold Standard, 1879-1913." Working Paper No. 6073, National Bureau of Economic Research, Cambridge, USA. 
Priestley, M.B. (1980a). Discussion of Tong and Lim (1980), 1980, pp. 273.

Priestley, M.B. (1980b). "State-Dependent Models: A General Approach to Non-linear Time Series Analysis." Journal of Time Series Analysis 1: 57-71.

Priestley, M.B. (1988). Nonlinear and Non-Stationary Time Series Analysis. Academic Press, London, UK.

Psaradakis, Z., M. Sola, and F. Spagnolo (2001). "On Markov Error-Correction Models." Working Paper, Birkbeck College, London, UK.

Psaradakis, Z., Sola, M., and F. Spangolo (2004a). "On Markov-switching Models, with an Application to Stock Prices and Dividends." Journal of Applied Econometrics 19: 69-88.

Psaradakis, Z., Sola, M., and F. Spangolo (2004b). Appendix of "On Markov-switching Models, with an Application to Stock Prices and Dividends." Journal of Applied Econometrics Data Archive, Available at: http://qed.econ.queensu.ca/jae/datasets/psaradakis002/pss-appendix.pdf.

R Development Core Team (2009). R: A Language and Environment for Statistical Computing. $R$ Foundation for Statistical Computing, Vienna, Austria. Available at: http://www.R-project.org.

Raj, B. (2002). "Asymmetry of Business Cycles: The Markov-Switching Approach." In A. Ullah, A.T.K. Wan, and A. Chaturvedi (eds): Handbook of Applied Econometrics and Statistical Inference, Dekker, New York, USA, pp. 687-710.

Rapsomanikis, G., D. Hallam, and P. Conforti (2003). "Market Integration and Price Transmission in Selected Food and Cash Crop Markets of Developing Countries: Review and Applications." In FAO: Commodity Market Review 2003-2004, Commodities and Trade Division of Food and Agriculture Organization of the United Nations, Rome, Italy, pp. 51-75.

Saikkonen, P. (2005). "Stability Results for Nonlinear Error Correction Models." Journal of Econometrics 127: 69-81.

Saikkonen, P. (2008). "Stability of Regime Switching Error Correction Models under Linear Cointegration." Econometric Theory 24: 294-318.

Samuelson, P. (1952). "Spatial Price Equilibrium and Linear Programming." American Economic Review 42: 283-303.

Schaller, H., and S. van Norden (2002). "Fads or Bubbles?" Empirical Economics 27(2): 335-362.

Seo, M.H. (2007). "Estimation of Non-Linear Error Correction Models." LSE STICERD Research Paper No. EM/2007/517, London School of Economics and Political Science, London, UK.

Seo, M.H., and O. Linton (2007). "A Smoothed Least Squares Estimator for Threshold Regression Models." Journal of Econometrics 141: 704-735.

Sephton, P.S. (2003). "Spatial Market Arbitrage and Threshold Cointegration." American Journal of Agricultural Economics 85: 435-450. 
Sercu, P., R. Uppal, and C. Van Hulle (1995). "The Exchange Rate in the Presence of Transaction Costs: Implications for Tests of Purchasing Power Parity." Journal of Finance 10: 1309-1319.

Serra, T., and B.K. Goodwin (2002). "Specification Selection Issues in Multivariate Threshold and Switching Models." In Proceedings of the AAEA \& WAEA Annual Meeting, Long Beach, USA, July 28-31.

Serra, T., and B.K. Goodwin (2003). "Price Transmission and Asymmetric Adjustment in the Spanish Dairy Sector." Applied Economics 35: 1889-1899.

Serra, T., and B.K. Goodwin (2004). "Regional Integration of Nineteenth Century U.S. Egg Markets." Journal of Agricultural Economics 55: 59-74.

Serra, T., J.M. Gil, and B.K. Goodwin (2006a). "Local Polynomial Fitting and Spatial Price Relationships: Price Transmission in EU Pork Markets." European Review of Agricultural Economics 33: 415-436.

Serra, T., B.K. Goodwin, J.M. Gil, and A. Mancuso (2006b). "Non-parametric Modelling of Spatial Price Relationships." Journal of Agricultural Economics 57: 501521.

Serra, T., D. Zilberman, J.M. Gil, and B.K. Goodwin (2008). "Nonlinearities in the US Corn-Ethanol-Oil Price System." Paper presented at AAEA Annual Meeting, Orlando, USA, July 27-29.

Sexton, R.J., C.L. Kling, and H.F. Carman (1991). "Market Integration, Efficiency of Arbitrage, and Imperfect Competition: Methodology and Application to U.S. Celery." American Journal of Agricultural Economics 73(3): 568-580.

Shepherd, A.W. (1997). Market Information Services. Theory and Practice. FAO Agricultural Services Bulletin 125, Rome, Italy.

Siklos, P.L., and C.W.J. Granger (1997). "Regime-Sensitive Cointegration with an Application to Interest-rate Parity." Macroeconomic Dynamics 1: 640-657.

Spiller, P.T., and C.J. Huang (1986). "On the Extent of the Market: Wholesale Gasoline in the Northeastern United States." Journal of Industrial Economics 5: 113-128.

Spiller, P.T., and R. Wood (1988). "The Estimation of Transaction Costs in Arbitrage Models." Journal of Econometrics 39: 309-326.

Stephens, E.C., E. Mabaya, S. von Cramon-Taubadel, and C.B. Barrett (2009). "Spatial Price Adjustment with and without Trade." Working paper, Pitzer College, Claremont, USA.

Stigler, G.J., and R.A. Sherwin (1985). "The Extent of the Market." Journal of Law and Economics 28(3): 555-585.

Stock, J.H. (1987). "Asymptotic Properties of Least Squares Estimators of Cointegrating Vectors." Econometrica 55: 1035-1056.

Stock, J.H., and M.W. Watson (1988). "Testing for Common Trends." Journal of the American Statistical Association 83: 1097-1107. 
Strachan, R., and B.A. Inder (2004). "Bayesian Analysis of the Error Correction Model." Journal of Econometrics 123: 307-325.

Subervie, J. (2008). "Rupture et Asymétrie dans la Transmission des Prix Agricoles Mondiaux." Paper presented at the International Conference on Market Integration and Food Security in Developing Countries, CERDI, CNRS-Université d'Auvergne, Clermont-Ferrand, France, November 3-4.

Takayama, T., and G. Judge (1971). Spatial and Temporal Price Allocation Models. North-Holland, Amsterdam, The Netherlands.

Tastan, H. (2003). "Simulation-Based Estimation of Spatial Price Equilibrium Models and Market Integration." Ph.D. Thesis, North Carolina State University, Raleigh, USA.

Tschernig, R. (2004). "Nonparametric Time Series Modeling." In H. Lütkepohl, and M. Krätzig (eds): Applied Time Series Econometrics., Cambridge University Press, Cambridge, USA, pp. 243-288.

Teräsvirta, T. (1994). "Specification, Estimation, and Evaluation of Smooth Transition Autoregressive Models." Journal of the American Statistical Association 89: 208218.

Teräsvirta, T. (2004). "Smooth Transition Regression Modeling." In H. Lütkepohl, and M. Krätzig (eds): Applied Time Series Econometrics, Cambridge University Press, New York, USA, pp. 222-242.

Tillmann, P. (2003). "The Regime-Dependent Determination of Credibility: A New Look at European Interest Rate Differentials." German Economic Review 4(4): 409431.

Tillmann, P. (2004). "Cointegration and Regime-Switching Risk Premia in the U.S. Term Structure of Interest Rates." Paper presented at the 2004 North American Summer Meeting of the Econometric Society, Brown University, Providence, USA.

Thomas, J.K. (2004). "Do Sunspots Produce Business Cycles?" Working paper, University of Minnesota, Minneapolis, USA.

Thompson, S., D. Sul, and M. Bohl (2002). "Spatial Market Efficiency and Policy Regime Change: Seemingly Unrelated Error Correction Model Estimation." American Journal of Agricultural Economics 84(4): 1042-1053.

Tjøstheim, D. (1986). "Some Doubly Stochastic Time Series Models." Journal of Time Series Analysis 7: 225-273.

Tong, H. (1978). "On a Threshold Model." In C.H. Chen (ed): Pattern Recognition and Signal Processing, Sijthoff and Noordhoff, Amsterdam, The Netherlands, pp. 575-586.

Tong, H. (1983). Threshold Models and Non-linear Time Series Analysis. SpringerVerlag, New York, USA.

Tong, H. (1990). Non-linear Time Series. A Dynamical System Approach. Clarendon Press, Oxford, UK. 
Tong, H. (2007). "Birth of the Threshold Time Series Model." Statistica Sinica 17: 8-14.

Tong, H., and K.S. Lim (1980). "Threshold Autoregression, Limit Cycles and Cyclical Data (with Discussion)." Journal of the Royal Statistical Society B42: 245-292.

Tostão, E., and B.W. Brorsen (2005). "Spatial Price Efficiency in Mozambique's Postreform Maize Markets." Agricultural Economics 33: 205-214.

Trenkler, C., and N. Wolf (2003). "Economic Integration in Interwar Poland - A Threshold Cointegration Analysis of the Law of One Price for Poland (1924-1937)." European University Institute Working Paper ECO No. 2003/5, San Domenico, Italy.

Trenkler, C., and N. Wolf (2005). "Economic Integration across Borders: The Polish Interwar Economy 1921-1937." European Review of Economic History 9: 199-231.

Tsay, R.S. (1989). "Testing and Modeling Threshold Autoregressive Processes." Journal of the American Statistical Association 84: 231-240.

Tsay, R.S. (1998). "Testing and Modeling Multivariate Threshold Models." Journal of the American Statistical Association 93: 1188-1202.

Ubilava, D., and M.T. Holt (2009). "Nonlinearities in the World Vegetable Oil Price System: El Nino Effects." Paper presented at the AAEA \& ACCI Joint Annual Meeting, Milwaukee, USA, July 26-28.

Uchezuba, D.I. (2005). "Measuring Market Integration for Apples on the South African Fresh Produce Market: A Threshold Error Correction Model." M.Sc. Thesis, University of the Free State Bloemfontein, South Africa.

Uppal, R. (1993). "A General Equilibrium Model of International Portfolio Choice." Journal of Finance 48: 529-553.

Weinstein, M. (1964). "The Sum of Values from a Normal and Truncated Normal Distribution." Technometrics 6: 104-105.

Whittle, P. (1954). "The Statistical Analysis of a Seiche Record." Journal of Marine Research 13: 76-100.

World Bank (2009). "Eastern Africa: A Study of the Regional Maize Market and Marketing Costs." Agriculture and Rural Development Unit (AFTAR), Sustainable Development Department, Country Department 1, Tanzania, Uganda and Burundi, Africa Region, Report No. 49831 - AFR, Washington D.C., US, December.

Yang, F., and S. Kant (2008). "Rent Capture Analysis of Ontario's Stumpage System Using an Enhanced Parity Bounds Model." Land Economics 84(4): 667-688. 
Appendices 


\section{Appendix A}

Spatial Market Integration in the EU Beef and Veal SeCtor: Policy Decoupling and the Outbreak of Blue Tongue DISEASE

This article is a cooperation with Bernhard Brümmer and Stanley R. Thompson. It has been published as discussion paper No. 0913 of the Department of Agricultural Economics and Rural Development of Georg-August-Universität Göttingen.

[http://www.unigoettingen.de/en/72592.html]

The article is accepted as presentation at the 114th Seminar of the EAAE "Structural Change in Agriculture: Modeling Policy Impacts and Farm Strategies", HumboldtUniversity of Berlin, Germany, April 15 - 16, 2010. 
The 2003 reforms of the Common Agricultural Policy of the European Union allowed for discretionary implementation among member states. Discretion was allowed with respect to the timing and the degree of decoupling of policy support. Differences among member states were particularly apparent in the European beef and veal sector. Using weekly data from 2003 to 2009 , we assess the consequences of different national implementation strategies of the reforms on market integration for young calves, which are intensively traded in the European Union. Time series properties are analyzed with a range unit-root test after which a multivariate cointegration model is estimated. We find that the calf markets in Germany, France, the Netherlands and Spain are integrated and tightly interrelated as evidenced by both short and long-run price transmission. We also find strong statistical support for the hypothesis that decoupling reduced calf price levels. Additionally, we ascertain that the outbreak of the Blue Tongue disease induced a structural change in parts of the EU calf market. Using counterfactual scenarios, we provide an indication of the cost involved with granting member states such a high degree of discretion in implementation. We conclude that the national markets studied here belong to a common market.

\section{Keywords}

2003 CAP reform, calf market, decoupling, EU, market integration, price transmission.

\section{Introduction}

Reforming the European Union's (EU) Common Agricultural Policy (CAP) has been an ongoing process. The first major effort was the MacSharry reform of 1992. This was followed by the Agenda 2000 and its mid-term review, which was eventually repackaged into the fundamental Fischler reforms of 2003. A key element of the 2003 reforms was decoupling, which aimed to sever the link between direct payments and production decisions. Unlike previous reforms, member states were allowed discretion over the timing and degree of decoupling. While differential implementation decisions were granted in almost all major European agricultural subsectors, they were most strongly apparent in the beef and veal sector of the Union. Since payments were no longer tied to the amount of slaughtered animals, decoupling impacted beef production profitability as different production incentives were provided. This transmitted to the calf markets in the form of a reduced willingness to pay for calves used in cattle or veal production, thus affecting the quantities and prices of animals traded. As the heterogeneity in the implementation of the reform can be expected to influence the relationships of prices in space, important implications for market integration are likely. In this paper, we seek to empirically explore how different policy choices impacted spatial price relationships and the degree to which price changes are transmitted between national calf markets in the long run. Furthermore, European cattle markets were subject to restrictions on animal transports which resulted from battling the outbreak of the Blue Tongue (BT) disease from 2006 onwards. Hence, we also regard potential effects of such trade restrictions, which peaked in Central Europe in late summer 2007. 
Within a multivariate cointegration framework, we use weekly price data from 2003 to 2009 to assess market interdependencies, market integration and price transmission among the four national EU markets of France, Germany, the Netherlands and Spain. While a large number of studies of market integration and price transmission have been carried out on U.S. and international agricultural markets (e.g., Rapsomanikis et al., 2003; Gonzalez-Rivera and Helfand, 2001), few have focused on intra-EU price relations (Zanias, 1993; Gordon et al., 1993; and Serra et al., 2006 are among the exceptions). We know of no recent investigation of the spatial price relationships among EU calf markets. Given the unique treatment of the beef and veal sector in the 2003 reforms, we are presented with an ideal situation for the study of how the effects of a changed policy environment impacted spatial price relationships. To our knowledge, we are the first to empirically assess the impact of policy decoupling on market integration.

The assessment of market integration represents an important means to study spatial market networks. The main interest lies in the question of whether price shocks emerged in one of the markets of the network are passed to the other markets so that trade flows which counteract the initial shock are triggered. Furthermore, consequences for consumers or producers are also of interest. If markets are not integrated, they do not share the same information set, in the sense that they are not driven by one and only one "pushing force" (Juselius, 2008, p. 88). In this case, price signals are not effectively passed through. Even if markets are integrated, price signals may spread only very slowly. Thus, since economic agents do not have complete information, welfare losses can result from the inefficient allocation of resources. Lacking integration of markets or weak transmission of price signals between them may be due to trade or domestic policies, exchange rate rigidities, or transactions costs. If the causes of these impairments are known, actions can be taken to improve the relationships of markets across space. Hence, the results can aid in the design of regional policy or trade policy. Moreover, evidence of well-functioning markets can help traders or policy makers in their markets assessments. On the other hand, policy makers and economists have strong interests in assessing the effects of certain policy measures in order to evaluate whether the actions adopted led to the desired consequences.

The CAP reforms agreed upon in 2003 were largely driven by concerns to re-integrate the common EU agricultural market into world markets. Additionally, national markets in the EU might not be well integrated even 20 years after the (nominal) completion of the common market. Hence, an empirical assessment of the degree of market integration seems promising. Furthermore, the distortion of price signals due to coupled support created a negative welfare impact in the past. Thus, the whole concept of decoupling agricultural support from current prices and production quantities aimed at reducing this impact. To gain a certain amount of freedom, member states negotiated with the EU Commission in choosing the degree of decoupling. Thus, the degree of decoupling in member states was somewhat different.

Market and price relationships can change due to major external shocks. In this context, the BT disease, which was first detected in Northern latitudes in August 2006, greatly impacted European cattle markets. The sample period studied covers the outbreak of the disease which falls near its midpoint. Hence, the time series analyzed are likely to 
contain structural breaks. Under such circumstances, standard unit root and cointegration tests are misleading. They inflate test statistics and suffer from considerable losses of power (Aparicio et al., 2006, and Gregory and Hansen, 1996). Consequently, we assess the time series data properties with a recently developed unit root test which is robust to potential structural breaks. We further seek to identify and to account for breaks in the cointegration relationships. The empirical results provide evidence that BT caused a structural break.

In the upcoming sections, the sample data is described. The post-2003 EU policy environment is also elaborated upon since some of the variables of the analysis are constructed based on these policies. We go further to describe the methodology used to assess market integration and price transmission. We also examine the EU slaughter calf market with the outlined methodology and discuss the empirical findings in detail. Finally we provide conclusions and policy implications.

\section{The Data and Policy Environment}

We use weekly post-2003 CAP reform data to investigate the dynamics and interrelationships in four major EU live calf markets. The four markets are: Germany (DE), France (FR), the Netherlands (NL) and Spain (ES) (Figure 1). This choice of countries is mainly motivated by their role in the EU calf trade. In Table 1, the Netherlands emerge as the largest importer of young calves in the EU. Spain is the second largest importer. Germany is a large net exporter, with a majority of its trade supplying the Netherlands. France ranks fourth in 2008 among the largest importers, but appears to be the largest exporter in the EU.

Table 1: Trade in Calves for the Top Two Exporters and Importers, 2008 ('000 Headcount)

\begin{tabular}{l|cccc}
\hline & Germany & France & Spain & The Netherlands \\
\hline Imports & 57 & 117 & 400 & 772 \\
Exports & 415 & 932 & 46 & - \\
\hline
\end{tabular}

Source: ZMP (2009a, 2009b).

The sample includes prices of young male calves aged eight days to four weeks from week 20 of 2003 to week 17 of 2009, i.e., 310 observations from May 15, 2003, to April 30, 2009. The weekly prices are collected by each member state and transmitted to the European Commission (European Commission, 2002). They are representative averages from each country's regions weighted by the relative importance of each breed and quality. In Figure 1, two observations stick out. First, possible seasonal patterns in the price series are discernible. Thus, seasonality must be considered in the model specification. Secondly, the German price is somewhat above those of France and the Netherlands for a large part of the sample period. This is mainly due to animal quality (breed) differences. France, and in particular the Netherlands, are heavily inclined towards less costly dairy calves. While we assume commodity homogeneity in the analysis, we recognize that different breeds and animal types exist among countries. The prices we use, however, are representative averages from each country's regions weighted by the relative importance of each breed. Animal numbers data suggest that 
the mixture of animals in each country has remained relatively constant over the sample period. Next, we describe the construction of the two important variables which are designed to quantify the decoupling policies and the appearance of BT.

\section{Policy variables}

The 2003 reforms in the beef and veal market eliminated the link between headage and payments; it was replaced with a single farm payment (SFP) which was based on historical entitlements between 2000 and 2002. While the aim of the reform was decoupling, individual member states had the option to either fully or partially decouple payments. If the SFP was partially implemented, farmers could apply for various slaughter premia: steers $150 €$ (two payments); bulls $210 €$, adult animals $80 €$ and calves $50 €$ per animal.

Figure 1: Weekly Calf Prices for Germany, France, the Netherlands and Spain

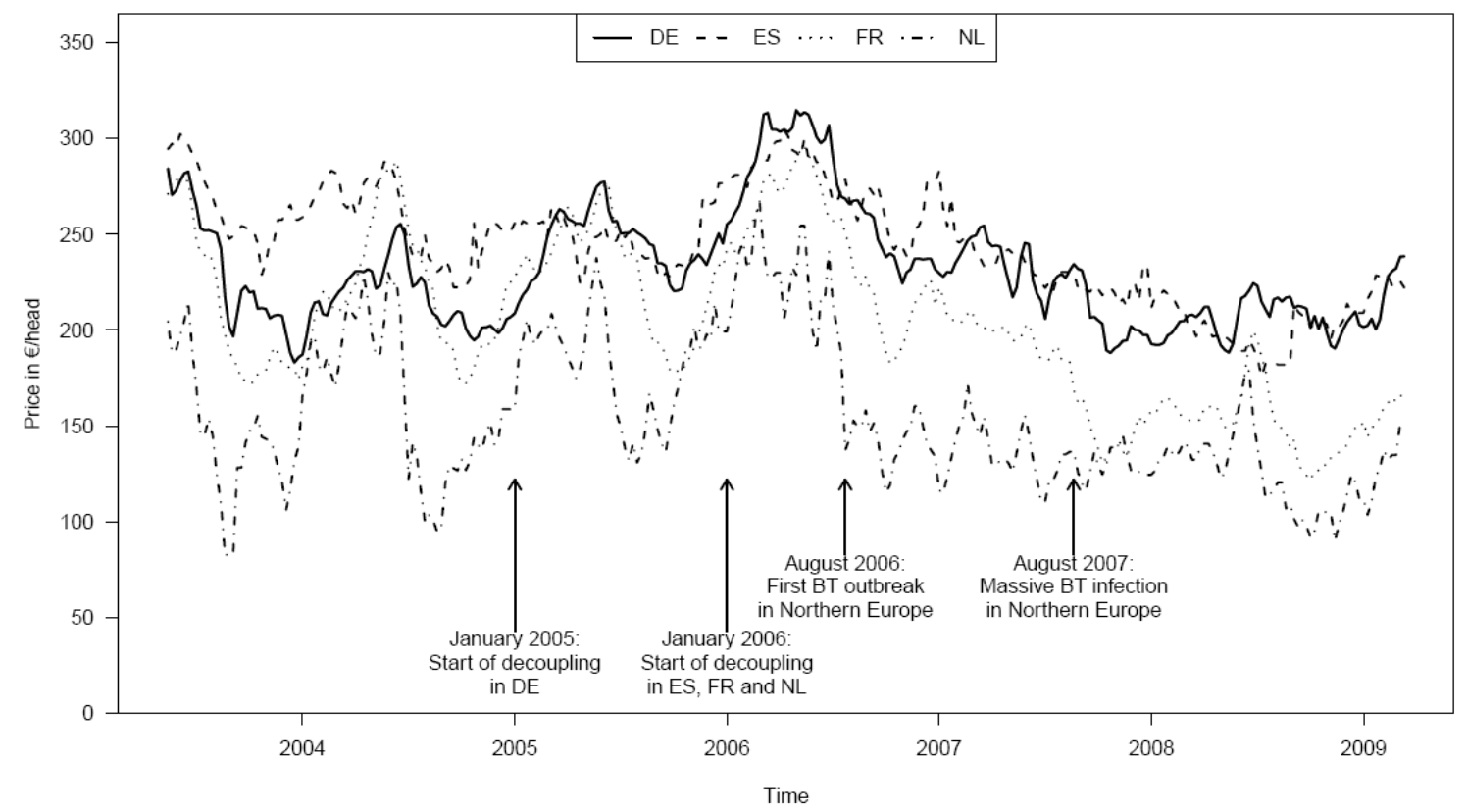

Source: European Commission (2009a).

The number of animals, i.e. bulls, steers, adult animals and calves, receiving slaughter premia each year, is reported by the European Commission (2009b). Using the values of the headage premia reported above, total annual monetary payments are computed for each country. Based on these numbers, we construct three policy indices pol $_{D E}$, pol $_{F R}$ and $p l_{N L}$ reflecting the degree of decoupling in Germany, France and the Netherlands, respectively ${ }^{1}$ (Table 2). The variables are constructed for each year between 2005 and

\footnotetext{
${ }^{1}$ We use the ${ }^{p o l} l_{F R}$ variable for both French and Spanish policies since both countries adopted virtually the same policy. For 2008 and 2009, no expenditure figures were available. Thus, we were forced to find a pragmatic approach for extrapolation because some variability in the policy variables is needed in order to avoid perfect multicollinearity. Animal numbers receiving premia are extrapolated by drawing from a normal distribution with mean and standard deviation of the animal numbers of 2006 and 2007 . We are aware that the chosen approach is rough. However, it suffices to meet the targets of giving meaningful estimates and allows for some variability in the animal numbers, which are known to closely resemble the numbers for the two previous years, but are not identical.
} 
2009 , relative to the average coupled payments in the base period 2002-2004. They are calculated for country $Z$ and year $t$ according to the formula

pol $_{Z}^{t}=\frac{1-\text { premia payed by } Z \text { in } t}{\text { average premia payed by } Z \text { in base period }}$

such that the greater the index value (the closer to 100), the higher the degree of decoupling, i.e., it can range from 0 (fully coupled) to 100 (fully decoupled).

Table 2: Policy Variables Quantifying the Degree of Decoupling

\begin{tabular}{l|ccccc}
\hline Year & $\mathbf{2 0 0 5}$ & $\mathbf{2 0 0 6}$ & $\mathbf{2 0 0 7}$ & $\mathbf{2 0 0 8}$ & $\mathbf{2 0 0 9}$ \\
\hline pol $_{D E}$ & 100 & 100 & 100 & 100 & 100 \\
pol $_{F R}$ & 7 & 77 & 78 & 78 & 77 \\
pol $_{N L}$ & 2 & 24 & 24 & 24 & 25 \\
\hline
\end{tabular}

Source: European Commission (2009b) and authors' calculations.

The SFP was implemented in Germany in 2005, while France, Spain and the Netherlands started one year later. Germany chose to fully decoupled payments in 2005 already. France and Spain partially decoupled in 2006 while the Netherlands decoupled payments also in the same year, but to a much limited extent. Slaughter premia for calves and adult animals partially remained in France, Spain and the Netherlands, whereas in Germany they were included in the SFP. As noted earlier, these different approaches are likely to yield different production incentives, since payments are differently linked to the production of beef in different countries.

Economic theory suggests an inverse relationship between decoupling and market prices. Beef production can be thought of as a function of a number of inputs, including young calves. The demand for calves is given by the marginal value product of calves in beef production. The headage premia is paid to the company delivering the cattle to the slaughterhouse, i.e. in most cases the cattle fattener. Since the premia is clearly coupled to production; the premia shifts the demand for calves, as an input, outward. If the premia are reduced or eliminated, the derived factor demand curve for calves shifts downward due to a reduction in the marginal value product of an additional calf. ${ }^{2}$ If the marginal cost of calf production does not change, the price of calves will fall. Thus, we expect a negative effect of the decoupling in a country to be reflected in its equilibrium price for calves.

\section{Blue Tongue variable}

Non-policy shocks can also impact market relationships. The animal disease BT was first reported in August 2006 with near simultaneous outbreaks in the Netherlands, Germany and France. BT is a seasonal non-contagious viral disease of ruminants mainly transmitted by a midge species that can cause mouth ulcers and in some cases a "blue tongue" in the animal (Conraths et al., 2009). It is prevalent in Sub-Saharan Africa, but has also been observed for many decades in the Mediterranean region. With

\footnotetext{
2 The approximate portion between 2002and 2004 of the monetary value of the total headage premium going to young calves is in Germany 2 percent, in France10 percent, in Spain 1 percent and in the Netherlands 20 percent. Source: European Commission (2009b).
} 
global warming, the disease spread northward and was first detected in Central Europe, specifically, the Southwest of the Netherlands in August 2006. It rapidly spread into the neighboring countries and in 2007, to the UK. The disease occurs in various versions (serotypes). Serotype 8 was the version of BT which first occurred in Central Europe. Other serotypes spread in the following months.

Although the number of animals infected with BT serotype 8 in Central Europe remained low in 2006, it became an important topic in the media. In August 2007 a massive outbreak was recorded in Germany, France and the Netherlands (Conraths et $a l ., 2009)$. Subsequently, the number of cases in Germany and the Netherlands declined due to the introduction of vaccination programs from 2008 onwards. Before January 2008, Spanish cattle were only infected with BT serotype 1; but later serotype 8 began to spread from the Southwest of France to the Northeast of the country.

Although fatality rates due to the disease are low for cattle, it has important consequences for the milk and cattle sectors. It reduces the fertility of cows, increases abortions and reduces dairy milk yields by up to 50 percent. Due to its potentially severe consequences for cattle, implications of the disease for calf prices are very likely.

Table 3 shows the means and standard deviations of prices before and after the peak outbreak of BT in August 2007. Both the means and standard deviations of prices were considerably lower in the period after than before.

Table 3: Prices ( $€ /$ Head) Before and After the Peak Number of Reported Blue Tongue Cases

\begin{tabular}{l|c|c|c|c}
\hline \multirow{2}{*}{} & \multicolumn{2}{|l|}{ Before August 2007 } & \multicolumn{2}{l}{ After August 2007 } \\
\cline { 2 - 5 } & Mean & Standard deviation & Mean & Standard deviation \\
\hline DE & 241 & 30 & 207 & 12 \\
ES & 223 & 21 & 152 & 14 \\
FR & 255 & 34 & 208 & 16 \\
NL & 167 & 40 & 127 & 18 \\
\hline
\end{tabular}

Source: European Commission (2009a).

In an effort to control the spread of the disease across the Union, the EU adopted strict control measures which included vaccinations and restrictions on the movement of cattle, sheep and goats (European Commission, 2007). When a confirmed case is identified, restriction and surveillance zones with radii 100 and $150 \mathrm{~km}$, respectively, are established (European Commission, 2000). Movement of animals out of the restricted zones is not allowed. Additionally, national import restrictions were occasionally issued by several member states, e.g., by France and Spain for German exports. However, Germany was able to continue calf export to the Netherlands, the most important destination of German calves. As both countries were subject to restricted zones of the same serotype, no movement restrictions applied and neither side issued national import restrictions. 


\section{Methodology and Economic Background}

According to Fackler and Goodwin (2001), a universally accepted definition remains allusive. In order to avoid ambiguities, we thus elaborate on the notions of market integration and price transmission. According to the Law of One Price (LOP), prices of a homogenous commodity in one market can differ by at most the costs $\tau_{t}^{X Y}$ of moving them from location $X$ to location $Y$. This condition is also termed the spatial arbitrage condition or the weak form of the LOP. If this relationship holds as an equality, then it is referred to as the strong form of the LOP, i.e., it holds then

$$
p_{t}^{Y}-p_{t}^{X}=\tau_{t}^{X Y}
$$

where $p_{t}^{X}$ and $p_{t}^{Y}$ denotes prices of a homogenous commodity in markets $X$ and $Y$ in time $t$. We investigate the existence of the strong form of the LOP; an equilibrium condition where price differences among markets evolve over time toward the transactions costs $\tau_{t}^{X Y}$ (Barrett, 2001). Since this notion is a long-run concept, prices can deviate from equality in the short-run due to various sources of shocks. When such a disequilibrium situation occurs, price signals will elicit the movement of products between surplus and deficit markets, thus restoring the long-run equilibrium.

The economic notion of equilibrium can be empirically investigated in the framework of cointegration analysis, where the cointegrating relationship is interpreted as the longrun equilibria. The existence of such a relationship implies a stationary term which is interpreted as the temporary and stochastic deviations from the equilibrium. The central characteristic of such a stationary series is that it frequently crosses its mean value. This property can also be interpreted as a long-run tendency towards the mean, i.e. the series does not drift apart from its mean value due to its stationarity. Clearly, such behavior closely corresponds to the economic understanding of equilibria, which is in itself a long-run concept.

If prices are found to be cointegrated, the system can be written as a vector errorcorrection model (VECM) as follows (Engel and Granger, 1987),

$$
\Delta p_{t}=\alpha \beta^{n} p_{t-1}+\sum_{i=1}^{k} \Gamma_{i} \Delta p_{t-i}+\varepsilon_{t}=\Pi p_{t-1}+\sum_{i=1}^{k} \Gamma_{i} \Delta p_{t-i}+\varepsilon_{t}
$$

where $p_{t}$ is a $n$-dimensional vector of prices of a homogenous product in $n$ spatially spread markets, and $\Delta p_{t}=p_{t}-p_{t-1}$. The matrix $\beta$ of dimension $n \times r$ contains the coefficients of $r$ linear combinations of the prices $p_{t}$. These combinations are interpreted as stationary long-run relationships between the prices. $\alpha$ denotes the $n \times r$ loading matrix containing the rates at which the price differences $\Delta p_{t}$ react on the deviations from the long-run equilibrium. These deviations, which are induced by short-term shocks to the market system, are quantified by $\beta^{\prime} p_{t-1}$. The matrix $\alpha$ contains hence relative shares at which the $j^{t h}, j=1, \ldots, r$ disequilibrium is adjusted for by each of the $n$ prices in each period, i.e., the adjustment speeds. The $n \times n$ matrices $\Gamma_{i}$ contain the short-run reactions of the price differences on past differences. $\varepsilon_{t}$ denotes a Gaussian white noise error term of appropriate dimension. 
Since calf trade among the four countries studied is likely to exhibit complex interdependencies, we adopt a multivariate approach. By considering all price series simultaneously in a single model, we overcome the omitted variable problem typical of pair-wise cointegration studies that have excluded relevant price series and errorcorrection terms.

As mentioned, a common shortcoming in the market integration literature is the inconsistent usage of terminology. Fackler and Goodwin (2001, p. 978) refer to market integration as "a measure of the expectation of the price transmission ratio". However, Barrett and $\mathrm{Li}$ (2002) define the concept as tradability of a commodity as either established by trade flows or the indifference of agents to trade. Our understanding comes closest to the definition of Gonzalez-Rivera and Helfand (2001, p. 576) who define it as "the set of locations that share both the same commodity and the same long run information". While we see market integration as a dichotomous quantity, price transmission is regarded as a gradual measure. The mere tradability condition does, in our opinion, not suffice to ensure that markets are integrated. For example, the setting in which a state trading agency uses prohibitive border protection measures to disconnect domestic from international markets, while still exporting domestic products, can hardly be viewed as integrated markets.

The theoretical conceptualization we adopt lends itself to a cointegration interpretation. A set of $n$ markets is called integrated if all of them are connected by either direct or indirect trade flows and if they are driven by one and only one common factor implying the existence of $r=n-1$ cointegration relationships in a system consisting of two prices each as also suggested in Fackler and Goodwin (2001). In this sense, market integration appears to be a dichotomous measure, that is, either $n-1$ long-run relationships and trade flows are exist among $n$ markets or not.

While market integration is a unique long-run concept, price transmission is, in our opinion, best viewed as having both a long- and a short-run dimension. Price transmission in the long-run is quantified by the slope parameters of the prices in a certain cointegration relationship $j, j \in\{1, \ldots, r\}$, i.e., by the $j$ th column of the cointegration matrix $\beta$. When using logged data, these parameters can be interpreted as long-run price transmission elasticities. Hence, long-run price transmission is a gradual measure since the respective $\beta$ coefficients can take continuous values around zero. The closer the measure is to zero, the weaker the price transmission in the long-run. In the special case in which these coefficients can be restricted to unity, the long-run price transmission is said to be complete. This implies that the price transmission elasticity does not statistically differ from one. Hence, a one percent change in one of the prices leads to a change of the same magnitude in the other price. Accordingly, the long-run aspect of price transmission is a gradual measure. The short-run dimension of price transmission refers to the sizes of the parameters in the $j$ th row of the loading matrix $\alpha$. They quantify the magnitudes to which each of the $n$ prices reacts on the $j$ th disequilibrium relationship from period to period, i.e. the speed of adjustment of a price shock. The sign of the respective parameters in $\alpha$ signals the direction of the adjustment while their absolute magnitude usually lies between 0 and 1 . Thus, price transmission in the short-run is also a gradual measure. Price transmission in the long-run can be complete, albeit slow in the short-run which illustrates that it is important to distinguish 
between these time horizons. Each of these characteristics describes one aspect of interrelationships of markets in space.

Design of the model

Based on the above considerations and test results, the final specification of the estimated VECM in (3) includes a number of variables. First, we augment the cointegration space by several variables: a constant, a time trend and the three policy variables $p o l_{D E}, p o l_{F R}$ and $p o l_{N L}$. Secondly, we include $k=2$ lags (AIC) of the price differences and a dummy variable for the year 2003 outside the cointegration space. With respect to the latter, there was a dramatic fall in calf prices in all countries during the first year of the sample period as a result of a number of exogenous events in the year 2003. These events include the ten country EU enlargement in early 2004. Another notable event was the response of calf prices to the peak in milk prices in 2002, which encouraged milk production and thus increased calf numbers some time after. Additionally, the Fischler reforms were not fully determined in early 2003. Seasonality was also included outside the cointegration space as significant seasonal patterns are suggested by Figure 1. Upon exploring this possibility, a likelihood-ratio test favored the inclusion of 52 weekly dummies.

\section{Empirical Results}

\section{Unit Root Tests}

A major challenge to the analyst is dealing with potential structural breaks in the univariate series and in the cointegration relationships. In our case, such a break may be due to the occurrence of BT. Standard unit root tests do not yield reliable results in the presence of breaks because their size and/or power are affected by the structural changes. To provide valid inference on the time series properties of the data, we adopt a recently published unit root test - the forward backward range unit root test (FB-RUR) developed by Aparicio et al. (2006). This nonparametric test offers an innovative approach to unit root testing. It counts the number of range extensions, i.e. the number of cumulative minima and maxima of the mean-adjusted time series. In contrast to a unit-root series (I(1)), a stationary series $(\mathrm{I}(0))$ is characterized by constant variance. This property translates into the fact that the number of range extensions will be small for a stationary series and large for a nonstationary series. The test statistic is small for $\mathrm{I}(0)$ series and large for $\mathrm{I}(1)$ processes. The test statistic is robust to data problems such as outliers or structural breaks. Whenever the test statistic is smaller than the critical value, the null hypothesis of a unit root is rejected.

Table 2 shows that all series, except the Dutch series, have a unit root. Although the Dutch series is found to be stationary, we regard it as nonstationary as recommended by Juselius $(2008$, p. 20). She argues that the unit root property of economic variables is very useful for the empirical analysis of long- and medium-run macroeconomic relationships. 
Table 4: Results of the FB-RUR Test

\begin{tabular}{l|cccc}
\hline Series & DE & ES & FR & NL \\
\hline FB-RUR statistic & 1.947 & 2.433 & 2.839 & $1.379 * * *$ \\
\hline Source: Authors' calculations. \\
$\begin{array}{l}\text { Note: The critical values for the } 5 \% \text { and } 1 \% \text { significance level are } 1.866 \text { and } 1.582, \text { respectively. Three asterisks denote significance } \\
\text { at the } 1 \% \text { level. }\end{array}$
\end{tabular}

\section{Cointegration Tests}

As mentioned above, also the cointegration relationships might be subject to structural breaks in this case. In such circumstances, standard cointegration tests such as the Johansen-trace-test or the ADF-test do not yield reliable results as the asymptotic distribution or the power of the test statistics are affected. The challenge of performing an adequate cointegration test under such circumstances is cumbersome since only few theoretical results have been obtained on this up to now, to our knowledge. Gregory and Hansen (1996) develop several tests which are valid in the presence of structural breaks in the intercept and/or slope of the cointegration relationship. These tests however, are only suitable for single cointegration relationships. The only tests applicable to a multivariate setting are a modified version of the Johansen-trace-test (Johansen, 1995) and the Saikkonen-Lütkepohl-test (Saikkonen and Lütkepohl, 2000). However, the limiting distribution of the Johansen-trace-test depends not only on deterministic terms in the cointegration relationship, but also on the number and the location of structural breaks, and has thus to be simulated for each individual case. The SaikkonenLütkepohl-test is shown to be independent of such nuisance parameters.

However, theoretical results have only been obtained for structural breaks in the constant. To our knowledge, neither empirical tests nor theoretical results are currently available on the behavior of multivariate cointegration test statistics in cases of breaks in the slope coefficients of the cointegration relationships or for the inclusion of exogenous variables in the cointegration space. Theoretical results on these two issues might yield an assessment of a wide range of interesting questions from an applied science point of view and are hence area for future research. Nevertheless, since we are particularly interested in how the long-run equilibrium is affected by a changing policy environment and an extensive outbreak of an animal disease, we adopt a pragmatic approach to obtain empirical evidence on these two issues.

Since the Johansen-trace-test is sensitive to structural breaks and deterministic variables in the cointegration relationship, we draw upon the Saikkonen-Lütkepohl-test since it is robust at least to breaks in the constants of the cointegration space (for all countries). Strong evidence for three cointegration relationships is found in the four-variate system. Hence, all cointegration relationships are bivariate. We conclude therefore that the four markets are integrated since we find $n-1=3$ cointegration relationships which means that the 4-variate system is driven by only one stochastic trend. 
Table 5: Results of the Saikkonen-Lütkepohl-cointegration-test

\begin{tabular}{l|cccc}
\hline H0 & $\operatorname{rank}(\Pi) \leq 0$ & $\operatorname{rank}(\Pi) \leq 1$ & $\operatorname{rank}(\Pi) \leq 2$ & $\operatorname{rank}(\Pi) \leq 3$ \\
\hline Test statistic & 81.55 & 35.97 & 14.26 & 0.71 \\
P-value & $<0.001$ & 0.001 & 0.022 & 0.455 \\
\hline
\end{tabular}

Source: Authors' calculations.

Since we suspect that the intercept of the cointegration relationships might be subject to structural breaks induced by the outbreak of BT, we test for this possibility. Due to the absence of more appropriate test procedures on the evidence of structural breaks, we use the Gregory-Hansen-test for the four-variate system with one cointegration relationship.

Table 6 displays the three test statistics for a structural break in the cointegrating relationship. We find significant structural breaks which fall on week 35 of 2007. This date closely corresponds to the peak outbreak of BT serotype 8. This is, indeed, strong evidence that the massive outbreak of BT impacted the long-run calf price relationships. Thus, we add a shift dummy $d_{A U G 07}$, which equals 1 for week 35/2007 up to the end of the sample period, into the cointegration space of the multivariate VECM. The fully specified VECM hence becomes

$$
\Delta p_{t}=\alpha \beta^{\prime}\left(p_{t-1}^{\prime} \quad \text { const trend } \operatorname{pol}_{D E} \quad \operatorname{pol}_{F R} \quad \operatorname{pol}_{N L} d_{A U G 07}\right)^{\prime}+\sum_{i=1}^{2} \Gamma_{i} \Delta p_{t-i}+\varepsilon_{t} \text {. }
$$

Table 6: Results of the Structural Break Gregory-Hansen-test

\begin{tabular}{cc|ccc}
\hline ADF* $^{*}$ statistic & $95 \%$ Critical value & Observation & Year & Week \\
\hline-5.29 & -5.28 & 224 & 2007 & 35 \\
\hline
\end{tabular}

Source: Authors' calculations.

\section{VECM Results}

We first estimate the unrestricted multivariate VECM (4) by the Johansen procedure (Johansen, 1995). We choose to normalize the bivariate cointegration relationships on DE, ES and FR, respectively, because the Netherlands was by far the largest importer of young calves among the four markets, as mentioned above. Hence, all long-run price equilibria are expressed with respect to the Dutch price. Based on the theoretical expectations and understanding of the beef and veal sector, we impose several overidentifying restrictions on the unrestricted model. First, we test the strong form of the LOP as formulated in (2). We find that the coefficients of the Dutch price can only be restricted in its relationships with the Spanish and French prices, respectively ( $p$-value of the according Wald test 0.11). Furthermore, German decoupling policy should not impact the ES-NL or the FR-NL relationships. However, the test for excluding the German price from the ES-NL relationship jointly with the other hypotheses is strongly rejected ( $\mathrm{p}$-value $<0.001$ ). The expectation that French/Spanish policy should not play a role in the DE-NL relation is confirmed by the joint test (Wald test p-value 0.133). Lastly, we expect the BT outbreak in 2007 to not have an impact on the DE-NL relationship since both countries were subject to restricted zones of the same serotype. 
Since no bilateral trade restrictions were issued, the movement of animals between both countries was not affected. The Wald test of this exclusion restriction, together with the not rejected hypotheses from before, yields a $\chi^{2}$-statistic of 8.05 which is not significant (p-value 0.153). Thus, we re-estimate the VECM with the restrictions imposed via a Generalized Least Squares (GLS) estimation as outlined in Lütkepohl and Krätzig (2004, pp. 103).

In addition, we impose restrictions on the adjustment and the short-run parameters. Obviously, economic theory can hardly provide clear hypotheses about each of the 252 parameters. We thus choose a statistical approach to identify valid restrictions. Using a sequential elimination of adjustment and short-run coefficients selected according to the largest reduction of the Hannan-Quinn criterion, we identify a set of 28 exclusion restrictions. The VECM is re-estimated with restrictions on the cointegration space, on the adjustment and on the short-run parameters via a two-stage procedure. This procedure uses the previous procedure for estimating the restricted cointegration relationships. In the second stage, it uses an estimated GLS estimator as discussed in Lütkepohl (2007, p. 197). A likelihood-ratio test indicates that these restrictions cannot be rejected ( $\mathrm{p}$-value 0.246 ).

We follow the recommendation of Hendry and Juselius (2001, p. 104), and identify several residuals as outliers by using identification criterion $\left|\hat{\varepsilon}_{t}\right|>3.3 \hat{\sigma}_{\hat{\varepsilon}}$. We include the eleven identified outliers as impulse or transitory dummies into the autoregressive part of the VECM. Misspecification tests applied to the residuals of this model version demonstrate that the chosen specification describes the data generating process adequately (Table 7).

Table 7: P-values of Misspecification Tests

\begin{tabular}{l|cccc}
\hline Series & LM-test & $\begin{array}{c}\text { Jarque-Bera } \\
\text { test }\end{array}$ & $\begin{array}{c}\text { ARCH- } \\
\text { LM test }\end{array}$ & $\begin{array}{c}\text { Multivariate } \\
\text { ARCH-LM test }\end{array}$ \\
\hline DE & & 0.4500 & 0.9440 & \\
ES & & 0.0008 & 0.2403 & \\
FR & & 0.1896 & 0.4319 & \\
NL & & 0.1005 & 0.9868 & 0.3935 \\
Multivariate test & 0.1676 & & & 0 \\
\hline Source: Authors'calculations
\end{tabular}

5 Interpretation

Table 8 displays the final estimates of the cointegration relationships for the restricted and outlier-corrected VECM. The coefficients of NL in the second column represent the long-run price transmission elasticities. The LOP in its strong form is only found to hold in the relationships between Spain and the Netherlands, and France and the Netherlands, respectively. Thus, we conclude that price transmission in the long-run is complete for these pairs. Only the price transmission elasticity of the German-Dutch relationship cannot be restricted to one. However, it is reasonably close to 1 . 
The magnitudes of the remaining coefficients are also plausible. The coefficients of the policy variables denote the average change of the price in the first column in response to increased decoupling. For example, an increase in decoupling in Germany by 10 percentage points is expected to result in a 0.7 percent decrease of the German calf price. Thus, decoupling led in each of the countries to price decreases. However, decoupling in the Netherlands had differing impacts on the calf prices in the various countries.

The estimated coefficients of the BT dummy are of plausible magnitude. They suggest that the massive outbreak of the disease in August 2007 as well as subsequent trade restrictions, which were issued as a result of the disease, indeed impacted spatial price relationships. These trade measures led to a near 14 percent drop in the Spanish price. This finding is plausible because while France was infected by serotype 8, Spain, as mentioned above, successfully curbed the spread for almost 1.5 years; the first case was detected in Spain only in January 2008. The BT dummy however, is not statistically significant in the French-Dutch relationship. Both countries suffered from the BT serotype 8 outbreak and thus belonged (partially) to the same restricted zone. Consequently, they were not subject to trade restrictions (European Commission, 2007).

Table 8: Cointegration Relationships of the Restricted VECM

\begin{tabular}{|c|c|c|c|c|c|c|c|}
\hline & NL & Constant & Trend & $p o l_{D E}$ & $p o l_{F R}$ & $p o l_{N L}$ & $d_{A D G O X}$ \\
\hline DE & $\begin{array}{c}1.173 \\
(0.055)\end{array}$ & $\begin{array}{l}-0.932 \\
(0.307)\end{array}$ & $\begin{array}{c}0.002 \\
(<0.001)\end{array}$ & $\begin{array}{l}-0.0007 \\
(<0.001)\end{array}$ & - & $\begin{array}{l}-0.006 \\
(0.003)\end{array}$ & - \\
\hline ES & $\begin{array}{c}1.000 \\
(-)\end{array}$ & $\begin{array}{c}0.349 \\
(0.082)\end{array}$ & $\begin{array}{c}0.002 \\
(<0.001)\end{array}$ & $\begin{array}{l}-0.0022 \\
(<0.001)\end{array}$ & $\begin{array}{l}-0.009 \\
(0.004)\end{array}$ & $\begin{array}{c}0.023 \\
(0.013)\end{array}$ & $\begin{array}{l}-0.135 \\
(0.037)\end{array}$ \\
\hline FR & $\begin{array}{c}1.000 \\
(-)\end{array}$ & $\begin{array}{c}0.116 \\
(0.124)\end{array}$ & $\begin{array}{l}>-0.001 \\
(<0.001)\end{array}$ & - & $\begin{array}{l}-0.013 \\
(0.004)\end{array}$ & $\begin{array}{c}0.039 \\
(0.013)\end{array}$ & $\begin{array}{c}0.014 \\
(0.036)\end{array}$ \\
\hline
\end{tabular}

Source: Authors' calculations.

Note: The prices in the first column are a function of the variables in the remaining columns with the reported coefficients. Standard errors are given below in parentheses.

Table 9 displays the estimated adjustment coefficients of the restricted and outliercorrected VECM. These estimates give information on how the national prices reacted to deviations from long-run price equilibria. The prices of each equilibrium show adjustment of the expected sign and are significant and of reasonable magnitude. The Dutch price appears to be weakly exogenous in the DE-NL and FR-NL relationships. Interestingly, several prices which are not part of the respective cointegration relationship show significant adjustment, e.g., the French price significantly responds to deviations from the DE-NL long-run equilibrium. This underscores the adequacy of the multivariate approach chosen; important variables would be omitted if the VECM would have been estimated for price pairs separately.

French and Dutch calf prices respond the fastest, i.e., correction of $50 \%$ of a shock (half live) taking place in approximately 5 to 6.5 weeks, while Spanish and particularly German prices react much slower with half-lives of up to 11 weeks. The French price is hence not only sensitive in the long-run to policy changes, but also shows a similar 
sensitivity regarding its reactions on deviations from the price equilibria in the shortrun. The general picture is that market prices quickly respond to disequilibria. Adjustment speeds vary between $6 \%$ up to more than $13 \%$ of equilibrium errors which means that at least half of a shock is adjusted within no more than 10 weeks $(2.5$ months). This finding shows that price transmission between the four calf markets is not only high in the long-run, but also occurs at high rates in the short-run.

Table 9: Adjustment Coefficients of the Restricted VECM

\begin{tabular}{l|ccc}
\hline $\begin{array}{l}\text { Cointegration } \\
\text { relationship }\end{array}$ & DE-NL & ES-NL & FR-NL \\
\hline DE & $-0.077[8.7]$ & - & $0.062[10.8]$ \\
& $(0.018)$ & $0.019)$ \\
ES & $0.062[10.8]$ & $-0.101[6.5]$ & - \\
& $(0.017)$ & $(0.020)$ & $-0.128[5.1]$ \\
FR & $0.102[6.5]$ & - & $(0.021)$ \\
& $(0.021)$ & $0.134[4.8]$ & - \\
NL & - & $(0.027)$ & \\
\hline
\end{tabular}

Source: Authors' calculations.

Note: Standard errors are given below the estimates in parentheses. Half-lives, defined as the time needed to correct c.p. $50 \%$ of any disequilibrium, in weeks are given in brackets. Note that these are hypothetical values since short-run parameters are not considered in the calculation.

\section{Counterfactual Simulations}

We conduct two counterfactual simulations which illustrate the effect of the decoupling on the equilibrium prices of each cointegration relationship. We compare estimated equilibrium prices based on the values of the observed policy variables at certain points in time to the hypothetical levels of these policy variables. The two scenarios presented are based on the 12-weekly Dutch average price before the respective date. Although the equilibrium prices are calculated for the pair-wise cointegration relationships of the restricted model, it has to be considered that the model coefficients were estimated in a multivariate system. They therefore encompass both the effects of a country's own policy choices on its domestic price and the effects of the policy choices of the other countries regarded in the system. Hence, a change in an equilibrium price cannot be interpreted as the sole consequence of the country's own decoupling choice, but the choices of the other countries also play a role.

Scenario I evaluates the situation for January 1, 2005. It compares the actual setting with a more conservative one by assuming that each of the four countries would have decided for zero decoupling. However, Germany took the most liberal policy decision and completely decoupled on this date even though this decision could have been delayed until January $2007^{3}$. Table 10 clearly shows that an increased degree of decoupling, which is equivalent to a decrease in coupled payments, had an expected depressing effect on the equilibrium price in each country. The variables of the actual

\footnotetext{
${ }^{3}$ The small actual policy variables in January 2005 of 7.5 percent in France and Spain and 1.6 percent in the Netherlands were due to the slightly lower animal numbers which received payments.
} 
policy choices are larger than the assumed ones. However, the equilibrium prices (A), based on the actual variables, are lower than the prices (B) in the hypothetical case of zero liberalization. The French equilibrium price appears to be the least impacted by the chosen decoupling policy. The German price would have been 8 percent higher without decoupling. In contrast, the Spanish equilibrium price could have been expected to be almost 30 percent higher if none of the countries would have decoupled.

Table 10: Scenario I - Fully Coupled Policies on January 1, 2005

\begin{tabular}{l|c|cccc}
\hline \multicolumn{1}{l|}{ Country } & DE & FR & ES & NL \\
\hline & $\begin{array}{l}\text { Observed price } \\
\text { €/head }\end{array}$ & 200 & 246 & 196 & 145 \\
\hline $\begin{array}{l}\text { Actual } \\
\text { policy }\end{array}$ & Policy variable & 100 & 7.5 & 7.5 & 1.6 \\
& Equilibrium price (A) & 151 & 156 & 198 & - \\
\hline Scenario I & Policy variable & 0 & 0 & 0 & 0 \\
& Equilibrium price (B) & 163 & 162 & 254 & - \\
& Ratio (B) to (A) & 1.08 & 1.04 & 1.28 & - \\
\hline
\end{tabular}

Source: Authors' calculations.

Scenario II assesses the hypothetical scenario of the most protective choice of a decoupling policy on January 1, 2007. This was the date of mandatory movement toward decoupling for all countries. With the exception of the Netherlands, chosen national policies were quite liberalized. However, we assume for this case that a relatively high degree of coupled support remained. The hypthotical values of the policy variables are set to 25 percent, roughly the observed situation in the Netherlands at this point in time. In

Table 11, it is apparent that the effects in Scenario II are much stronger than two years earlier in Scenario I. Equilibrium prices would have been much higher if Germany, France and Spain had opted for considerably more conservative policy choices ${ }^{4}$. Again, effects of decoupling are least for the German calf price and strongest for the French price.

\footnotetext{
${ }^{4}$ At first glance, the hypothetical equilibrium prices for ES seem very high as does the margin between the German and Spanish prices. We must emphasis that the equilibrium prices will never be observed in practice. As Table 12 in the appendix shows, the estimated deviations from equilibrium, e.g. for ES-NL, lie between -0.43 and 0.73 . Hence, observed prices might well have been $300 € /$ head for example since $434 € \cdot \mathrm{e}^{-0.3}=322 €$.
} 
Table 11: Scenario II - Most Protective Policy Choice by January 1, 2007

\begin{tabular}{l|l|cccc}
\hline \multicolumn{2}{l|}{ Country } & DE & FR & ES & NL \\
\hline & Observed price & 235 & 254 & 206 & 143 \\
& $€ /$ head & & & & \\
\hline Actual policy & Policy variable & 100 & 78 & 78 & 24 \\
& Equilibrium price (C) & 164 & 144 & 227 & - \\
\hline Scenario II & Policy variable & 25 & 25 & 25 & 25 \\
& Equilibrium price (D) & 171 & 304 & 434 & - \\
& Ratio (D) to (C) & 1.04 & 2.12 & 1.91 & - \\
\hline
\end{tabular}

Source: Authors' calculations.

\section{Dynamic Analysis}

A common way to assess system dynamics is to examine impulse response functions. However, impulse responses have been shown to have a number of weaknesses in a multivariate system (Pesaran and Shin, 1996). The main drawback is that the estimated functions are not unique. They depend on the ordering of the variables and the chosen orthogonalization of shocks. Lee and Pesaran (1993) suggest an alternative measure. They propose considering time paths which track the effect of a system-wide shock to the cointegration relations. They call such system-wide impulse responses persistence profiles. Persistence profiles are defined as "the scaled difference between the conditional variances of the $n$-step and the (n-1)-step-ahead forecasts" (Pesaran and Shin, 1996). Formally, scaled persistence profiles for multiple cointegration vectors are defined as:

$h_{Z}(n)=G H_{Z}(n) G=G \hat{\beta}^{\prime} \hat{B}_{n} \hat{\Omega} \hat{B}^{\prime}{ }_{n} \hat{\beta} G$.

$H_{z}(n)$ denotes the unscaled persistence profile of an $n$-step-ahead forecast of a unitshock to the multivariate system. It is calculated from estimated cointegration vector $\hat{\beta}$ where deterministic terms restricted to the cointegration space (constant, trend and dummies) are not regarded. Hence, the profiles are independent of time. Furthermore, the recursive sum of the estimated parameter matrices $\hat{B}_{n}$ of the World representation of the multivariate process is considered. Lastly, the estimated variance-covariance matrix $\hat{\Omega}$ of the shocks of the World representation plays a role. The World representation is approximated by the corresponding VAR form of the estimated VECM. Hence, the $\hat{B}_{n}$ matrices in (5) are functions of $\hat{\Pi}$ and $\hat{\Gamma}_{i}, i=1,2$. The matrix $G$ denotes a suitable scaling matrix. The resulting time profiles are unique and independent of the ordering of the variables and the orthogonalization of shocks. They are functions of the forecast horizon $n$, and converge eventually to zero for cointegrated models; although the convergence can take a while (Garrat et al., 2006). Figure 2 displays the persistence profiles of the restricted VECM estimated above.

The time paths of the three cointegration relationships are very similar. After overshooting in the first week after the system-wide shock, i.e. temporarily increased 
disequilibrium, the profiles converge rapidly to zero. Within four weeks, more than 50 percent of any shock is absorbed into each of the cointegration relationships. The French and the Spanish relationships although overshooting the most, show the steepest decline afterwards. After eight weeks, only 6 percent of the shock remains in the DENL and FR-NL relationships, respectively. In the ES-NL relationship, more than $98 \%$ of the shock is absorbed after eight weeks. This finding confirms the general picture of the close interrelationships of the four European calf markets studied.

Figure 2: Persistence Profiles of the Restricted Model

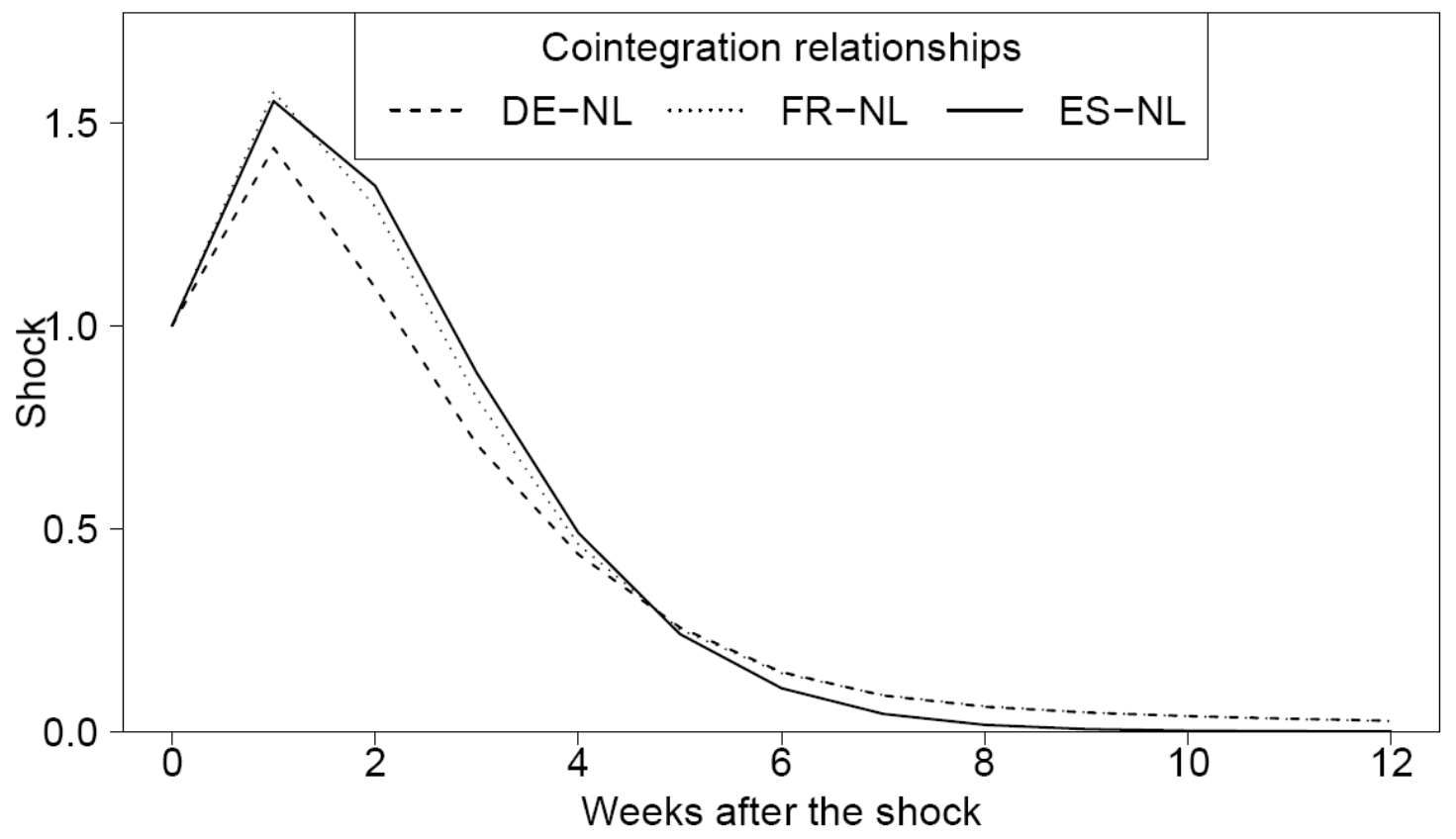

Source: Authors' calculations.

\section{Conclusions}

Following the 2003 Fischler reforms of the EU's Common Agricultural Policy, decoupling of support payments from production was implemented differently by EU member states. In the beef and veal sector, German policy makers opted for the most liberal choice of full decoupling in January 2005 while Spain, France and the Netherlands initiated partial decoupling a year later. Decoupling reduces slaughter premia for cattle, which in turn, reduces the marginal value product of calves in beef production. Hence, such a policy can be expected to lead to decreasing prices for calves as the derived demand curve shifts downward. Since 2003 however, European cattle markets were not only subject to changing policy, but also to a major animal health crisis induced by the first outbreak of the Blue Tongue disease in Central Europe. In August 2007, a large scale outbreak occurred, bringing strict restrictions on animal movements for some states.

In this paper, we empirically explore how these external forces impacted the degree of long-run price transmission between four major European calf markets. We analyze interdependencies of calf markets by using weekly price data of young male calves of 
Germany, France, the Netherlands and Spain from 2003 to 2009. A recently developed range unit-root test, which is, among other features, robust to structural breaks, and a multivariate vector error-correction model are used for this end. We conceptually differentiate between the notions of market integration and price transmission. The former term is seen as a dichotomous measure while and the latter concept is a gradual measure of both a long- and a short-run dimension. We find strong evidence for the existence of three cointegration relationships among the four prices. Thus, the markets can be regarded as integrated. Most of the estimated coefficients are of plausible sign and magnitude. Price transmission in the long-run is found to be complete in two of the three relationships. Long-run price transmission was significantly impacted by decoupling policies. The outbreak of the Blue Tongue disease played a significant role in the Spanish-Dutch long-run relationship. Price transmission in the short-run price is found to be fast.

The estimation results are illustrated by two counterfactual scenarios which demonstrate the price depressing effects of decoupling in comparison with hypothetical scenarios of more conservative liberalization strategies. Both scenarios show that the policy choices of decoupling indeed lowered the expected equilibrium prices in all national markets studied.

Dynamic analysis of the analyzed system confirms the general picture of the multivariate estimation. We compute persistence profiles, which show the reaction path along time for each of the long equilibria, to absorb a system wide shock. The estimated time paths underpin the findings of tightly interrelated prices of the spatially separated markets. Within a period of less than four weeks, more than half of any shock is absorbed into the system. We conclude that the four calf markets studied are closely interconnected and find strong evidence that they belong to a common European market. 


\section{References}

Aparicio, F., A. Escribano and A. Sipols (2006). "Range Unit-Root Tests: Robust against nonlinearities, Error Distributions, Structural Breaks and Outliers." Journal of Time Series Analysis 27: 545-576.

Barrett, C.B. and J.R. Li (2002). "Distinguishing Between Equilibrium and Integration in Spatial Price Analysis." American Journal of Agricultural Economics 84: 292-307.

Conraths, F.J., J.M. Gethmann, C. Staubach, T.C. Mettenleiter, M. Beer and B. Hoffmann (2009). "Epidemiology of bluetongue virus serotype 8, Germany." Emerging Infectious Diseases 15(3): 433-435.

Engle, R. F. and C.W.J. Granger (1987). "Cointegration and Error Correction: Representation, Estimation and Testing." Econometrica 55: 251-276.

European Commission (2000). COUNCIL DIRECTIVE 2000/75/EC. Available at http://eurlex.europa.eu/. Accessed in May 2009.

European Commission (2002). COMMISSION REGULATION (EC) No 2273/2002. Available at http://eur-lex.europa.eu/. Accessed in May 2009.

European Commission (2007). COMMISSION REGULATION (EC) No 1266/2007. Available at http://eur-lex.europa.eu/. Accessed in May 2009.

European Commission (2009a). Directorate General Agriculture, beef and veal, market prices for live animals. Available at:

http://ec.europa.eu/agriculture/markets/beef/privi/index.htm. Accessed in May 2009.

European Commission (2009b). Economic data on the implementation of the common agricultural policy. Available at:

http://ec.europa.eu/agriculture/agrista/2008/table_en/en361.htm, tables 3.6.1.10, 3.6.1.11, and 3.6.1.12. Accessed in March 2009.

Fackler, P.L. and B.K. Goodwin (2001). "Spatial Price Analysis". In: B.L. Gardner and G.C. Rausser, eds., Handbook of Agricultural Economics. Amsterdam: Elsevier Science, pp. 971-1024.

Garrat, A., K. Lee, M.H. Pesaran and Y. Shin (2006). "Global and National Macroeconomic Modelling: A Long-Run Structural Approach.” Oxford: Oxford University Press.

Gonzalez-Rivera, G. and S.M. Helfand (2001). "The Extent, Pattern, and Degree of Market Integration: A Multivariate Approach for the Brazilian Rice Market." American Journal of Agricultural Economics 83: 576-592.

Gordon, D.V., J.E. Hobbs and W.A. Kerr (1993). "A test for price integration in the EC lamb market." Journal of Agricultural Economics 44: 126-134.

Gregory, A.W. and B.E. Hansen (1996). "Residual-based Tests for Cointegration in Models with Regime Shifts." Journal of Econometrics 70: 99-126.

Hendry, D.F. and K. Juselius (2001). "Explaining Cointegration Analysis: Part II.” The Energy Journal 22(1): 75-120.

Johansen, S. (1995). "Likelihood-based Inference in Cointegrated Vector Autoregressive Models." Oxford: Oxford University Press.

Juselius, K. (2008). "The Cointegrated VAR Model: Methodology and Applications." New York: Oxford University Press.

Lee, K.C. and M.H. Pesaran (1993). "Persistence profiles and business cycle fluctuations in a disaggregated model of UK output growth." Richerche Economiche 47: 293-322. 
Lütkepohl, H. (2007). "New Introduction to Multiple Time Series Analysis.” Berlin: Springer.

Lütkepohl, H., and M. Krätzig (2004). "Applied Time Series Econometrics." Cambridge: Cambridge University Press.

Pesaran, M.H. and Y. Shin (1996). "Cointegration and speed of convergence to equilibrium." Journal of Econometrics 71: 117-143.

R Development Core Team (2008). "R: A language and environment for statistical computing." $R$ Foundation for Statistical Computing, Vienna, Austria. Available at www.rproject.org. Accessed in May 2008.

Rapsomanikis, G., D. Hallam and P. Conforti (2003). "Price transmission in selected food and cash crop markets of developing countries: Review and Applications." In: Commodity Market Review 2003-2004. Commodities and Trade Division of FAO, Rome.

Saikkonen, P. and H. Lütkepohl (2000). "Testing for the cointegration rank of a VAR process with structural shifts." Journal of Business \& Economic Statistics 18: 451-464.

Serra, T., J.M. Gil and B.K. Goodwin (2006). "Local polynomial fitting of spatial price relationships: price transmission in EU pork markets." European Review of Agricultural Economics 33: 415-436.

Zanias, G. P. (1993). "Testing for integration in European Community agricultural product markets." Journal of Agricultural Econonomics 44: 418-427.

ZMP (2009a). Zentrale Markt- und Preisberichtsstelle. Infografik 2009/416, Kälberhandel in Europa 2008.

ZMP (2009b). Zentrale Markt- und Preisberichtsstelle. Press release „Probleme im Kälberhandel“", 18.02.2009. Available at:

http://www.agrimarkt.de/infos/zmp/agrarmaerkte/fleisch/. Accessed in April 2009. 


\section{Appendix}

Table 12: Descriptive Statistics of the Estimated Error-Correction Terms

\begin{tabular}{l|ccc}
\hline & DE-NL & ES-NL & FR-NL \\
\hline Minimum & -0.1419 & -0.4334 & -0.1317 \\
Median & 0.2490 & -0.0029 & 0.2190 \\
Maximum & 1.1004 & 0.7324 & 0.7570 \\
\hline
\end{tabular}

Source: Authors' calculations.

Note: For calculating the observed magnitudes of relative price deviations from equilibrium, the value of the exponential function of the estimated residuals has to be considered. 


\section{Appendix B}

Markov-Switching Estimation of Spatial Maize Price TransMission PROCESSES BETWEEN TANZANIA AND KENYA

This article is a cooperation with Stephan von Cramon-Taubadel and Sergiy Zorya. It has been published in the American Journal of Agricultural Economics 91 (Number 5 - Proceedings Issue, 2009): 1432-1439.

The article was presented in the invited paper session "New Econometrics Methods for Structural Change Analysis of Agricultural and Food Markets" at the 2009 AAEA \& ACCI Joint Annual Meeting, Milwaukee, Wi, USA, July 26- 28, 2009. 
We discuss the Markov-switching vector autoregressive (MS-VAR) class of non-linear time series models which can be used to analyze recurring discrete structural changes in time series. Hamilton's (1989) seminal Markov-switching (MS) model of the U.S. business cycle triggered considerable interest in the MS approach in economics. Most empirical applications to date have focused on the business cycle and financial markets, but we see potential for MS-VAR models in agricultural economics for example in price transmission analysis. In the following we first provide an overview of the MSVAR framework. We then present an illustrative application to maize price transmission between Tanzania and Kenya. The article closes with a discussion of strengths, weaknesses, and potential uses of the MS-VAR approach in price transmission analysis.

\section{A Brief Overview of MS-VAR Models}

Following Krolzig (1997), the basic idea behind the MS-VAR class of models is that the parameters of a VAR process are allowed to depend on an unobserved regime variable $s_{t} \in\{1, \ldots, M\}$, representing $M$ possible states of the world. In its most general form, the MS-VAR is given by:

(1) $y_{t}=v\left(s_{t}\right)+\sum_{j=1}^{p} A_{j}\left(s_{t}\right) y_{t-j}+u_{t}, \quad u_{t} \sim \operatorname{NID}\left(0, \Sigma\left(s_{t}\right)\right)$,

where $y_{t}=\left(y_{1 t}, \ldots, y_{K t}\right)^{\prime}, t=1, \ldots, T$ is a $K$-dimensional time series vector, $v\left(s_{t}\right)$ and $A_{j}\left(s_{t}\right)$ are matrices of intercepts and autoregressive (AR) parameters of appropriate dimension, and $\Sigma\left(s_{t}\right)$ is the variance-covariance matrix of a Gaussian zero-mean error process $u_{t}$. In (1) the terms $v\left(s_{t}\right), A_{j}\left(s_{t}\right)$, and $\Sigma\left(s_{t}\right)$ describe the dependence of the respective parameters on the unobserved regime $s_{t}$. The intercept in (1) for example will be regime-dependent as follows:

(2) $v\left(s_{t}\right)=\left\{\begin{array}{c}v_{1} \text { if } s_{t}=1 \\ \vdots \\ v_{M} \text { if } s_{t}=M .\end{array}\right.$

Hence, the non-linear data generating process in (1) can be described as piecewise linear (i.e. as linear conditional on the regimes).

Depending on which parameters in (1) are allowed to be regime-dependent, different sub-classes of the MS-VAR result. Krolzig (1997) proposes the terminology MSx(M)$\operatorname{VAR}(p)$ to distinguish between them, where $M$ is the number of regimes, $p$ the order of the VAR, and $x$ indicates which parameters are regime-dependent. Thus, a $\operatorname{MSI}(M)$ $\operatorname{VAR}(p)$ refers to a model in which the intercept is regime-dependent, while $\operatorname{MSA}(M)$ $\operatorname{VAR}(p)$ and $\operatorname{MSH}(M)-\operatorname{VAR}(p)$ refer to regime-dependence in the AR terms $A_{j}\left(s_{t}\right)$ and the error covariance $\Sigma\left(s_{t}\right)$, respectively. These elements can be combined, so that the 'full-blown' model in (1) can be considered a $\operatorname{MSIAH}(M)-\operatorname{VAR}(p)$. 
A further sub-class of MS-VAR models which is not immediately apparent from (1) is the $\operatorname{MSM}(M)-\operatorname{VAR}(p)$ in equation (3):

(3) $y_{t}-\mu\left(s_{t}\right)=\sum_{j=1}^{p} A_{j}\left(y_{t-j}-\mu\left(s_{t-j}\right)\right)+u_{t}, \quad u_{t} \sim \operatorname{NID}(0, \Sigma)$,

where the second ' $M$ ' in MSM refers to 'mean-adjusted.' MSM-models can also include regime-dependent AR and error covariance parameters. MSM-VAR and MSIVAR models imply quite different time series behavior; while the mean of each time series jumps in the MSM-VAR immediately to its new level after a regime change, it approaches the new level smoothly in the MSI-VAR (Krolzig 1997).

Finally, the MS-VAR can be re-specified into vector error-correction form (MSVECM). In this specification the loading parameters can also be regime-dependent, along with the intercept, AR, and error covariance parameters (Krolzig 1997). The great variety of possible specifications points to one of the major advantages of the MS-VAR model class, which is its flexibility in capturing different types of structural change in time series.

To complete the MS-VAR, it is necessary to specify the process that governs the evolution of the regimes $s_{t}$. In MS-models $s_{t}$ is governed by a hidden Markov chain with a discrete and finite number of regimes in discrete time. This Markov chain is assumed to be ergodic and irreducible, meaning that a stationary distribution of regimes exists, and any regime can be reached from any other regime (i.e. there is no 'absorbing' regime). The evolution of $s_{t}$ is assumed to be characterized by the transition matrix:

(4) $P=\left(\begin{array}{ccc}p_{11} & \cdots & p_{1 M} \\ \vdots & \ddots & \vdots \\ p_{M 1} & \cdots & p_{M M}\end{array}\right)$,

where $p_{i j}=\operatorname{Pr}\left(s_{t+1}=j \mid s_{t}=i\right)$ is the probability of switching from regime $i$ in time $t$ to regime $j$ in $t+1$. As the evolution of $s_{t}$ is assumed to satisfy the Markov property $\operatorname{Pr}\left(s_{t+1} \mid s_{t}, s_{t-1}, \ldots, y_{t}, y_{t-1}, \ldots,\right)=\operatorname{Pr}\left(s_{t+1} \mid s_{t}\right)$, whereby the probability of $s_{t+1}$ depends only on $s_{t}$ and $P$, the resulting regime generating process is memoryless (i.e. the past has no influence on the future except through the present). The Markov chain is typically assumed to be homogenous (i.e. to have constant transition probabilities $p_{i j}$ ).

MS-VAR models are most often estimated using the expectation maximization (EM) algorithm. The EM algorithm uses an initial estimate of the model parameters to estimate the probabilities of being in each of the $M$ unobserved regimes $s_{t}$ (smoothed probabilities). These probabilities are then used to update the estimated parameter vector. These steps are repeated until a convergence criterion is satisfied. The EM algorithm converges relatively quickly to the neighborhood of the mode of the likelihood function and is robust to local maxima and singularities in this function; however, once in the neighborhood of the mode, convergence can be slow (Mizrach and 
Watkins 1999). Recent publications (e.g. Frei 2008) explore the use of Bayesian techniques to estimate MS-VAR models.

If the number of regimes $(M)$ in a Markov-switching model is known, then the EM algorithm produces consistent parameter estimates, and likelihood-ratio-tests can be used to generate statistical inferences on these parameters. If the number of regimes is not known, the problem of testing $M$ vs. $M+1$ regimes arises. Since some model parameters are not defined under the null, standard maximum-likelihood inference is not possible. Several solutions to this problem are proposed in the literature, including the use of complexity-penalized likelihood criteria (e.g. Smith, Naik, and Tsai 2006) and tests based on quasi-likelihood ratios (Cho and White 2007), but model selection remains a challenge in the MS-VAR framework.

Several extensions of the MS-VAR framework described above have been proposed. Some authors (e.g. Jerzmanowski 2006) relax the assumption of constant transition probabilities by allowing them to be functions of exogenous variables such as economic fundamentals. Pelagatti (2008) proposes a duration dependent MS-VAR in which the transition probabilities depend on how long the Markov chain has been in a particular regime. Krolzig (1997) and Kim (2009) go a step further to allow for endogenous regime switching by letting the transition probabilities be a function of $y_{t}$.

\section{An Application to Maize Price Transmission between Tanzania and Kenya}

\section{Motivation in the Context of Price Transmission Analysis}

The insight that price transmission is likely to be regime dependent, for example due to shifts in transaction costs or trade flow interruptions and reversals, has led to the use of a variety of non-linear modeling techniques. These include threshold VECM (Goodwin and Piggott 2001), smooth transition regression (Mainardi 2001, Serra et al. 2008, Ubilava and Holt 2009), and parity bounds models (Barrett and Li 2002).

An important characteristic of these approaches is that they assume that regime shifts are triggered by observable variables, for example the magnitude of the error-correction term in the threshold VECM. In some settings, however, regime shifts depend on variables that are unobservable or not amenable to measurement. For example Brümmer, von Cramon-Taubadel, and Zorya (2009) conjecture that price transmission in the Ukrainian wheat/flour chain is affected by policy interventions that are not always announced officially or enforced evenly over time. The behavior of actors in the chain might change due to such interventions (or threats thereof), leading to changes in price transmission. In such cases the MS-VAR framework might be able to generate useful insights into the timing and nature of otherwise unobservable regime shifts.

\section{Background and Data}

To illustrate the use of MS-VAR models in price transmission analysis, we consider maize market integration between Tanzania and Kenya. Maize is an important staple food in these countries, and the Tanzanian government in particular intervenes on maize markets in pursuit of improved food security. Interventions include the operation of strategic grain reserves and restrictions on the movement of grain between regions 
within the country or on exports to neighboring countries (Aliguma et al. 2008). One of the most visible and presumably most influential measures is the export ban (Temu, Manyama, and Temu 2007). The government in Kenya also intervenes on maize markets; a recent overview and analysis of these policies is provided by Jayne, Meyer, and Nyoro (2008).

Figure 1 depicts the maize price margins between Nairobi (Nai) in Kenya and four markets in Tanzania: Dar es Salaam (Dar), Arusha (Aru), Mbeya (Mbe), and Songea (Son). The margins are calculated using five corresponding series of monthly wholesale maize prices from January 2000 through September 2008 (105 observations) in US\$/t obtained from the Regional Agricultural Trade Intelligence Network of the Eastern Africa Grain Council (RATIN 2008). The margins are much higher in some periods than in others, which may be an indication of regime-dependent behavior. Figure 1 also illustrates the periods over which export bans have been in place according to Temu, Manyama, and Temu (2007).

\section{Figure 1: Maize Price Margins and Tanzanian Export Bans}

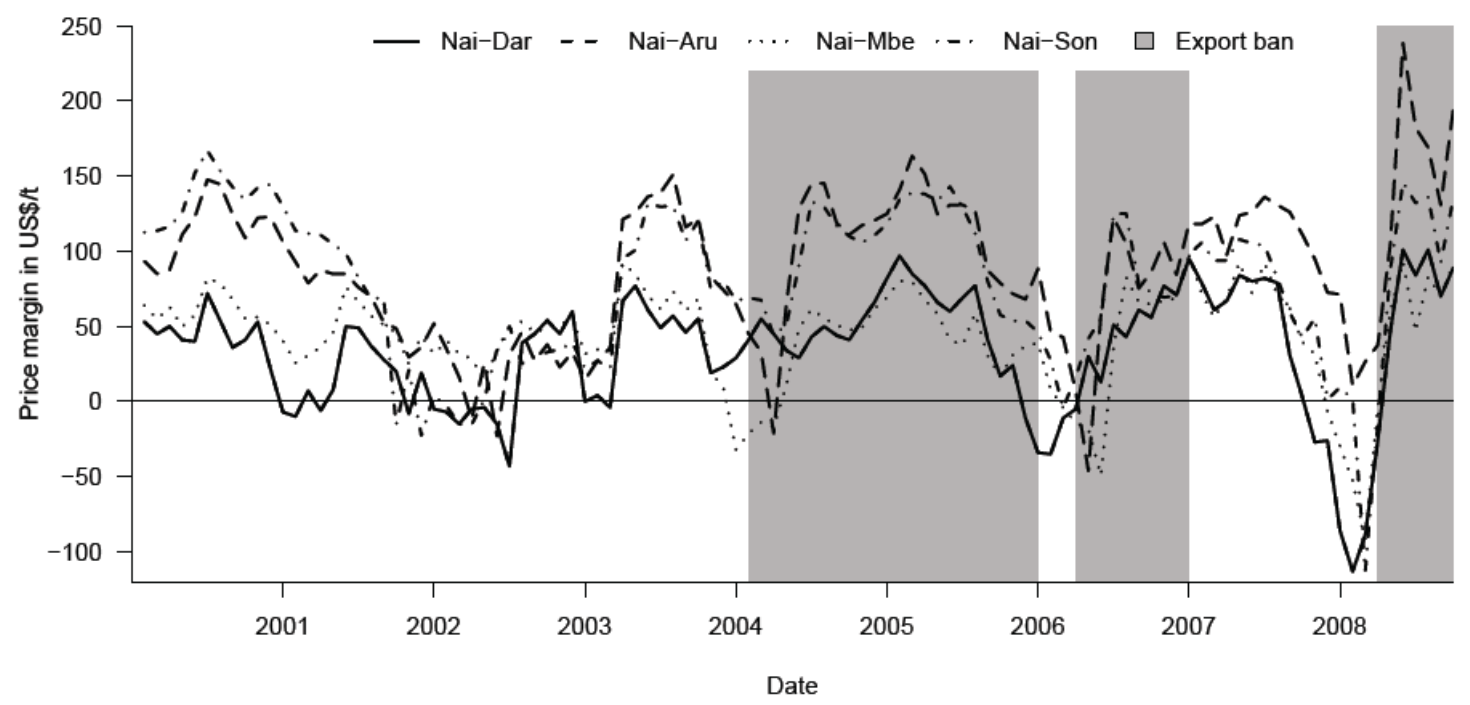

Source: RATIN (2008), and Temu, Manyama, and Temu (2007).

Export bans are expected to increase the Kenya-Tanzania price margin by decreasing the supply in Kenya and increasing it in Tanzania, all other things being equal. However, figure 1 shows that the correspondence between high price margins and export bans is not one-to-one. For example margins were high in 2000, 2003, and the first half of 2007, although no exports bans were reported. Against this background, we estimate a MS-VAR using the price margins in figure 1 to assess their potential regimedependent behavior and the correspondence to periods of known export bans.

\section{Model Selection and Estimation}

First, we study the data for evidence of nonlinearities by analyzing the residuals of a linear VAR of the four margins. The VAR rather than the VECM specification is chosen because the null hypothesis of a unit root is rejected for all four margins by the Augmented Dickey-Fuller test. We employ the Hannan-Quinn Criterion (HQ) and the 
Schwarz Criterion (SC) for model selection, both of which point to 1 lag. The residuals of the resulting VAR(1) display autocorrelation and non-normality (table 1). Increasing the lag order to 3 as indicated by the Akaike Information Criterion solves the first problem but not the second. Since non-normal residuals can be a consequence of failure to account for non-linearity such as regime-dependency (Lütkepohl and Krätzig 2004), we analyze whether there is evidence of non-constant parameters in the VAR(1). A bootstrapped Chow forecast test of the null hypothesis that all model parameters are constant is rejected at the 5 percent level over almost the entire sample period. Since figure 1 suggests that the margin levels shift over time, and this is what export bans are expected to produce, we focus on the intercepts of the VAR(1). Figure 2 presents recursive estimates of the intercept of the Nairobi-Arusha margin and suggests that it is indeed subject to shifts at several points in time over the sample period. Results for the other intercepts of the VAR(1) are similar.

Table 1: Residual Analysis of Linear VAR(1) and VAR(3) Models (p-Values)

\begin{tabular}{l|lllll}
\hline \multirow{2}{*}{ Model } & $\begin{array}{l}\text { Portmanteau } \\
\text { 12 lags }\end{array}$ & $\begin{array}{l}\text { Breusch-Godfrey } \\
\text { 5 lags }\end{array}$ & $\begin{array}{l}\text { Normality }^{b} \\
\text { Skewness }\end{array}$ & Kurtosis & Joint \\
\hline VAR(1) & 0.472 & 0.001 & $<0.001$ & $<0.001$ & $<0.001$ \\
VAR(3) & 0.576 & 0.207 & $<0.001$ & $<0.001$ & $<0.001$ \\
\hline
\end{tabular}

Source: Authors`calculations

${ }^{a}$ Breusch-Godfrey LM test for autocorrelation.

b Test for multivariate normality (Doornik and Hansen 1994).

Therefore, we next investigate whether a MS-VAR is able to provide an improved representation of the maize price margins. Both the HQ and the SC favor a meanadjusting specification that includes regime-dependent heteroskedasticity (MSMHVAR). Considering two regimes to capture phases with and without interference in trade such as export bans appears reasonable, but the possibility of three regimes is explored as well. Hence, we estimate $\operatorname{MSMH}(2)-\operatorname{VAR}(p)$ and $\operatorname{MSMH}(3)-\operatorname{VAR}(p)$ specifications using the MSVAR package for Ox (Krolzig 2004). Up to $p=5$ lags in the autoregressive part are considered. The HQ identifies the MSMH(3)-VAR(1) as best, while the SC favors the MSMH(2)-VAR(1) (Table 2). 
Figure 2: Recursive Estimates of the Intercept of the VAR(1) for the NairobiArusha Maize Price Margin

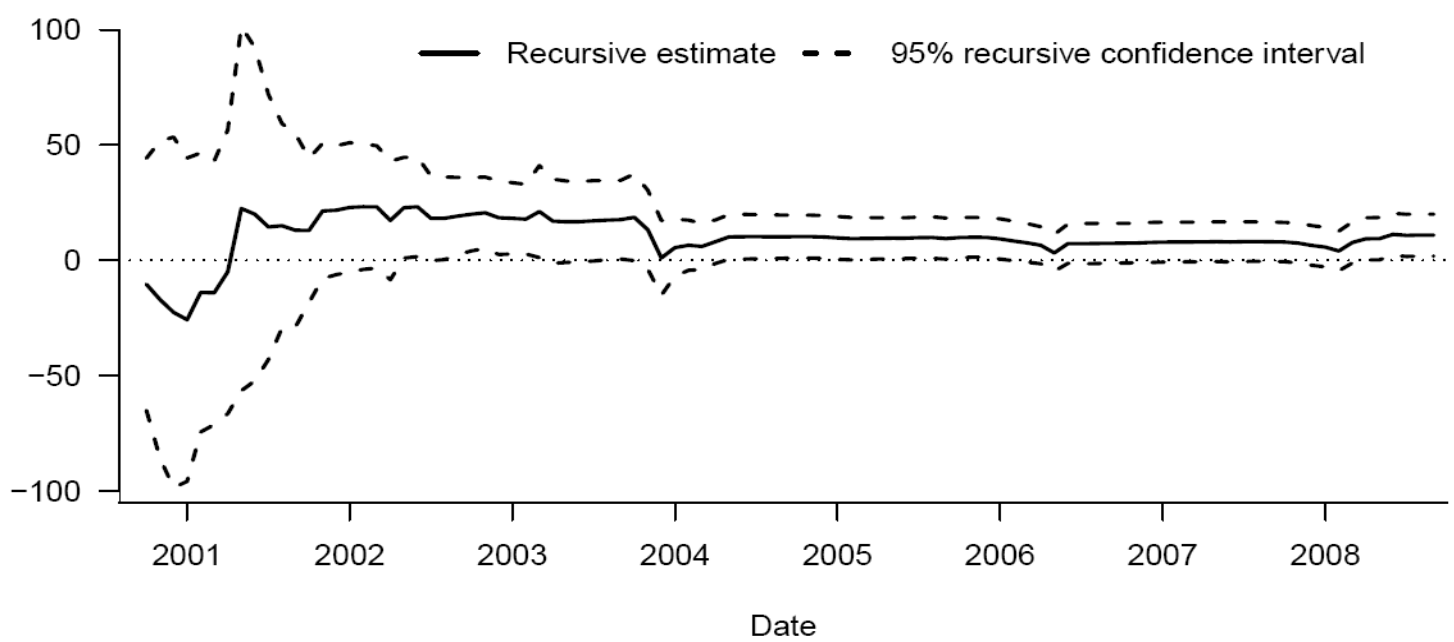

Source: Authors`calculations.

The residuals of both models are non-normal and conditionally heteroskedastic. Inspection of the residuals reveals four outliers according to the criterion $\left|\hat{\varepsilon}_{t}\right|>3.3 \hat{\sigma}_{\varepsilon}$; these outliers are common to both the two- and three-regime MSMH specifications and occur in the Nairobi-Arusha margin in May and June 2006, in the Nairobi-Mbeya margin in February 2008, and in the Nairobi-Songea margin in May 2008. Including dummy variables for these observations eliminates non-normality and heteroskedasticity (table 2). Furthermore, it reduces both the HQ and SC criteria and leads both of them to favor the MSMH(2)-VAR(1) specification.

Table 2: Selection Criteria and Residual Tests (p-Values) for MSMH(2)-VAR(1) and MSMH(3)-VAR(1) Specifications with and without Dummies for Outliers

\begin{tabular}{|c|c|c|c|c|}
\hline & \multicolumn{2}{|c|}{ MSMH(2)-VAR(1) } & \multicolumn{2}{|c|}{ MSMH(3)-VAR(1) } \\
\hline & $\begin{array}{l}\text { Without } \\
\text { dummies }\end{array}$ & $\begin{array}{l}\text { With } \\
\text { dummies }\end{array}$ & $\begin{array}{l}\text { Without } \\
\text { dummies }\end{array}$ & $\begin{array}{l}\text { With } \\
\text { dummies }\end{array}$ \\
\hline HQ & 35.963 & 35.116 & 35.818 & 35.639 \\
\hline SC & 36.658 & 36.054 & 36.786 & 36.639 \\
\hline Portmanteau & 0.373 & 0.706 & 0.697 & 0.523 \\
\hline Heteroskedasticity $^{\mathrm{a}}$ & 0.001 & 0.884 & $<0.001$ & 0.952 \\
\hline Normality ${ }^{\mathrm{b}}$ & $<0.001$ & 0.707 & 0.040 & 0.322 \\
\hline
\end{tabular}

Source: Authors `calculations.

${ }^{a}$ Test for multivariate conditional heteroskedasticity (Doornik and Hendry 1996).

$\mathrm{b}$ Test for multivariate normality (Doornik and Hansen 1994). 


\section{Discussion}

The preferred MSMH(2)-VAR(1) specification is parsimonious. It points to the existence of two regimes distinguished from one another by different levels of mean price margins between Kenya and Tanzania. These differences are large, with mean margins in the 'high' regime more than double those in the 'low' regime and error variances roughly 50 percent lower in the 'high' than in the 'low' regime (table 3 ). The model distinguishes sharply between the 'high' and 'low' regimes, producing smoothed probabilities that are close to 0 or 1 over the entire sample period, and it divides the sample period into a sequence of clearly distinct regime episodes (figure 3). The transition probabilities indicate that both the 'high' and the 'low' margin regimes are highly persistent; the probability of remaining in 'high' from one period to the next is 92 percent, and the probability of remaining in 'low' is 89 percent.

Table 3: Regime Means and Standard Deviations from the Estimated MSMH(2)$\operatorname{VAR}(\mathbf{1})$

\begin{tabular}{l|l|llll}
\hline Regime & Statistic & $\begin{array}{l}\text { Nairobi - Dar } \\
\text { es Salaam }\end{array}$ & $\begin{array}{l}\text { Nairobi - } \\
\text { Arusha }\end{array}$ & $\begin{array}{l}\text { Nairobi - } \\
\text { Mbeya }\end{array}$ & $\begin{array}{l}\text { Nairobi - } \\
\text { Songea }\end{array}$ \\
\hline \multirow{2}{*}{ 'High' } & Mean & 60.3 & 65.7 & 110.8 & 117.3 \\
& Std. error & 13.8 & 11.9 & 12.7 & 13.8 \\
\hline \multirow{2}{*}{ 'Low' } & Mean & 20.5 & 20.7 & 48.6 & 54.4 \\
& Std. error & 24.8 & 19.4 & 18.8 & 30.2 \\
\hline
\end{tabular}

Source: Authors`calculations.

Figure 4 compares the estimated 'high' margin episodes with known export bans, according to the literature. While there is some overlap since 2004, the MS-VAR identifies 'high' regime episodes in 2000-2001, 2003, and 2007 that do not correspond to known export bans. Several explanations for this lack of correspondence can be proposed. First, the information on the implementation of export bans in Temu, Manyama, and Temu (2007) might be incomplete, especially for earlier years. Second, exports might have been reduced or curtailed in some periods even in the absence of an official export ban, for example by unofficial export restrictions or by restrictions on movements of grain within Tanzania from production regions to regions that border on Kenya. Third, changes in domestic supply and demand balances in Kenya and Tanzania, both seasonally and from year to year, can be driving periods of high and low margins along with interference in movements and trade of maize. For example sustained high margins in 2007 despite the end of the export bans implemented in mid2006 could reflect continued high prices in Kenya following a severe drought in 2006 (FewsNet 2007). The results of the MS-VAR estimation presented here can guide future efforts to collect data on these possible explanations, with a view towards improving our understanding of the determinants of price transmission between Tanzania and Kenya. 
Figure 3: Smoothed Probabilities of the 'high' and 'low' Regimes, and Regimedependent Means of the Margins

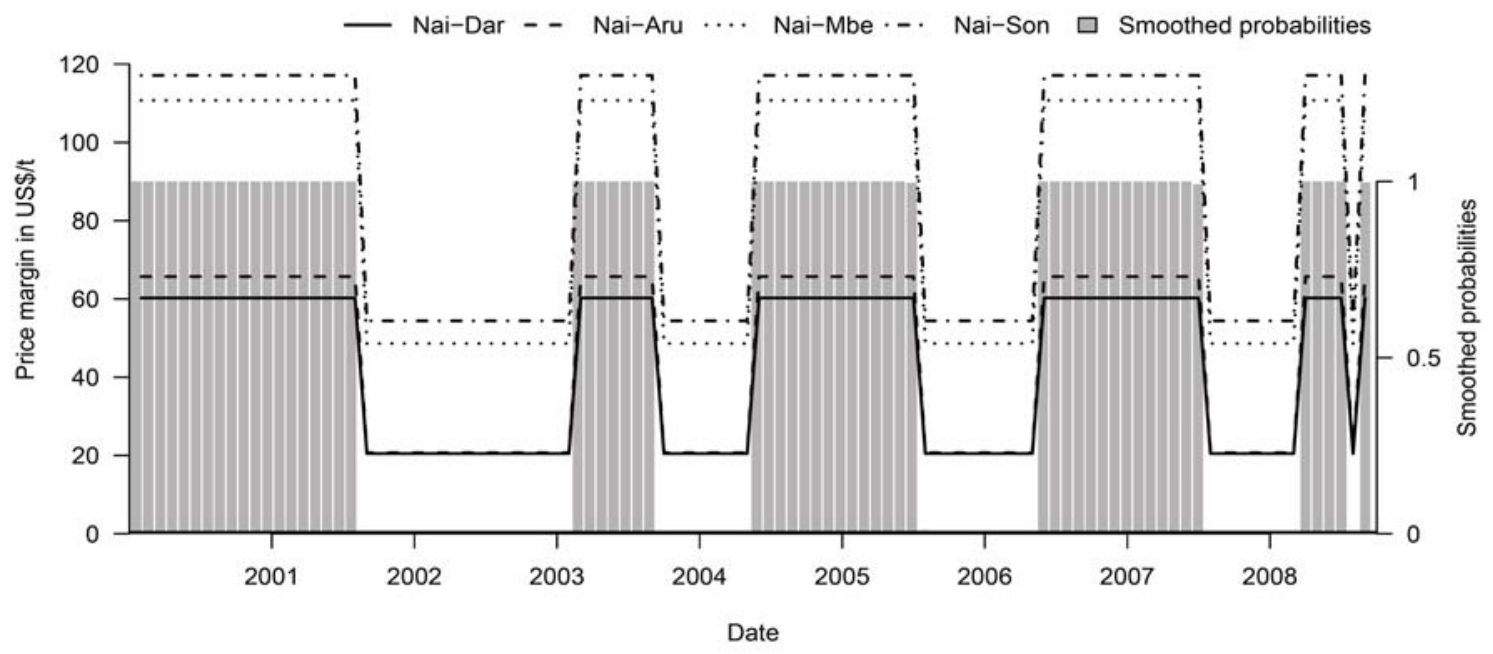

Source: Authors`calculations.

\section{Outlook and Conclusions}

We present Markov-switching vector autoregressive models as a tool for analyzing recurring structural changes in time series. This model class is widely used in business cycle and financial market analysis due to its considerable flexibility. We argue that it can be a useful tool in agricultural economics, too, in cases in which regime-dependent behavior is suspected, but the variables governing regime shifts are unobservable or unknown. Even in settings in which such a variable is observable, contrasting the results of other non-linear modeling techniques with MS models might produce useful insights. For example while the presence or absence of trade flows is expected to influence price transmission between two markets, there is sometimes reason to believe that the available data on trade flows is flawed (e.g. due to smuggling). In this case contrasting the results of models that use this trade data with the results of a MS-model might be useful.

The empirical application to maize margins between Kenya and Tanzania presented above illustrates the use of MS-VAR models in such a context. The estimated MSMH(2)-VAR(1) identifies several high-margin episodes that correspond well with known periods of Tanzanian export bans, but also several additional high-margin episodes during which no export bans were known to have been in effect. The results of the MS-VAR estimation presented here can guide future efforts to collect additional data with a view towards improving our understanding of the determinants of price transmission between Tanzania and Kenya.

An important advantage of the MS-VAR framework is its great flexibility in modeling regime-dependent time series behavior. The associated danger is that of over-fitting, especially since no generally valid model selection procedure in MS-VAR models has been established. Hence, practitioners run the risk of estimating spurious regressions in spurious regimes, producing results in sample that provide a misleading view of the 
underlying data generating process. Subject to these dangers, MS-VAR techniques can be a very useful addition to the existing toolbox.

Figure 4: Episodes of the Estimated 'high' Margin Regime and Export Bans According to the Literature

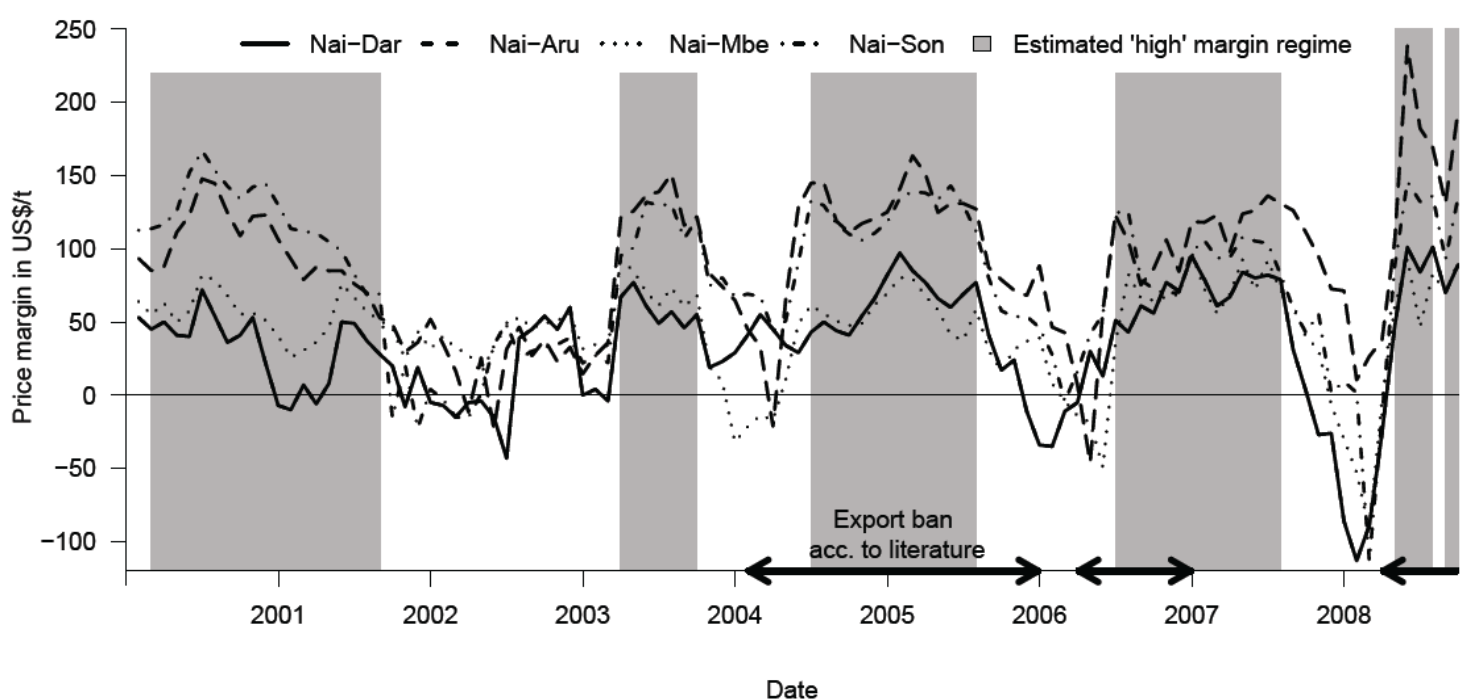

Source: RATIN (2008), Temu, Manyama, and Temu (2007), and authors`calculations. 


\section{References}

Aliguma, L., G. Ashimogo, G.S. Mwale, J. Nyoro, A. Phiri, and L.N. Traub. 2008. "Strengthening Food Security in Sub-Saharan Africa through Trade Liberalization and Regional Integration. Country Annexes to Report 1. Maize Market Sheds in Eastern and Southern Africa.” Working paper, Michigan State University, East Lansing.

Barrett, C.B., and J.R. Li. 2002. "Distinguishing between Equilibrium and Integration in Spatial Price Analysis." American Journal of Agricultural Economics 84(2):292-307.

Brümmer, B., S. von Cramon-Taubadel, and S. Zorya. 2009. "The Impact of Market and Policy Instability on Price Transmission between Wheat and Flour in Ukraine.” European Review of Agricultural Economics 36(2):203-230.

Cho, J.S., and H. White. 2007. "Testing for Regime Switching." Econometrica 75(6):1671-1720.

Doornik, J.A., and D.F. Hendry. 1996. PcGive 9.0 for Windows and PcFiml 9.0 for Windows, vol. 2. London: International Thomson Business Press.

Doornik, J.A., and H. Hansen. 1994. "A Practical Test of Multivariate Normality.” Unpublished, Nuffield College.

Famine Early Warning Systems Network (FewsNet). 2007. Kenya Food Security Update. Accessed: January 2009.

Available at: http://www.fews.net/docs/Publications/Kenya_200612en.pdf.

Frei, L. 2008. “The Markov-switching Vector Error Correction Model - Dynamics, Bayesian Inference, and Application to the Spot and Forward Swiss Franc - US Dollar Exchange Rates.” PhD dissertation, University of Basel.

Goodwin, B.K., and N.E. Piggott. 2001. "Spatial Market Integration in the Presence of Threshold Effects.” American Journal of Agricultural Economics 83(2):302317.

Hamilton, J.D. 1989. “A New Approach to the Economic Analysis of Nonstationary Time Series and the Business Cycle.” Econometrica 57(2):357-384.

Jayne, T.S., R.J. Meyer, and J. Nyoro. 2008. "The Effects of NCPB Marketing Policies on Maize Market Prices in Kenya.” Agricultural Economics 38(3):313-325.

Jerzmanowski, M. 2006. "Empirics of Hills, Plateaus, Mountains and Plains - A Markov-switching Approach to Growth.” Journal of Development Economics 81(2):357-385.

Kim, C.-J. 2009. "Markov-switching Models with Endogenous Explanatory Variables II - A Two-step MLE Procedure.” Journal of Econometrics 148(1):46-55.

Krolzig, H.-M. 1997. Markov-Switching Vector Autoregressions. Modelling, Statistical Inference, and Applications to Business Cycle Analysis. Berlin: Springer.

Krolzig, H.-M. 2004. "MSVAR - An Ox Package Designed for the Econometric Modelling of Univariate and Multiple Time Series Subject to Shifts in Regime.” Unpublished, Nuffield College. 
Lütkepohl, H., and M. Krätzig. 2004. Applied Time Series Econometrics. Cambridge: Cambridge University Press.

Mainardi, S. 2001. "Limited Arbitrage in International Wheat Markets: Threshold and Smooth Transition Cointegration," Australian Journal of Agricultural and Resource Economics 45(3):335-360.

Mizrach, B., and J. Watkins. 1999. “A Markov Switching Cookbook.” In P. Rothman, ed. Nonlinear Time Series Analysis of Economics and Financial Data. Dordrecht: Kluwer, pp. 33-43.

Pelagatti, M.M. 2008. "Duration Dependent Markov-Switching Vector Autoregression: Properties, Bayesian Inference and Application to the Analysis of the U.S. Business Cycle.” In T. Nagakawa, ed. Business Fluctuations and Cycles. Hauppauge NY: Nova Science Publishers, pp.43-66.

R Development Core Team. 2008. R: A Language and Environment for Statistical Computing. Vienna: R Foundation for Statistical Computing. Available at: http://www.r-project.org.

Regional Agricultural Trade Intelligence Network of the Eastern Africa Grain Council (RATIN). 2008. Accessed: November 2008. Available at: http://www.ratin.net/.

Serra, T., D. Zilberman, J.M. Gil, and B.K. Goodwin. 2008. "Nonlinearities in the US corn-ethanol-oil price system.” Paper presented at AAEA annual meeting, Orlando FL, 27-29 July.

Smith, A., P.A. Naik, and C.-L. Tsai. 2006. "Markov-switching Model Selection Using Kullback-Leibler Divergence.” Journal of Econometrics 134(2):553-577.

Temu, A., A. Manyama, and A. Temu. 2007. “Alternative Staple Food Trade and Marketing Policy Interventions for Tanzania: A Country Level Assessment of the Maize Sub-Sector.” Draft Report prepared for FAO, Dar es Salaam, Tanzania.

Teräsvirta, T. 2004. "Smooth Transition Regression Modeling.” In H. Lütkepohl, and M. Krätzig, eds. Applied Time Series Econometrics. Cambridge: Cambridge University Press, pp. 222-242.

Ubilava, D. and M.T. Holt. 2009. "Nonlinearities in the World Vegetable Oil Price System: El Nino Effects". Paper presented at AAEA \& ACCI joint annual meeting, Milwaukee WI, 26-28 July. 


\title{
Appendix C
}

\author{
Grenzeffekte in Der MarkTintegration bei Mais in Ost- \\ AFRIKA: EINSICHTEN AUS EINEM SEMI-PARAMETRISCHEN \\ REGRESSIONSMODELL
}

This article is a cooperation with Joseph Amikuzuno, Stephan von Cramon-Taubadel and Sergiy Zorya. It has been published in the Tagungsband GEWISOLA 2009 - 49. Jahrestagung der Gesellschaft für Wirtschafts- und Sozialwissenschaften des Landbaus e.V., Kiel, Germany.

[http://www.uni-kiel.de/gewisola2009/beitrage/v4korrigiert.pdf]

The article was presented at the GEWISOLA 2009 - 49. Jahrestagung der Gesellschaft für Wirtschafts- und Sozialwissenschaften des Landbaus e.V. "Agricultural and food markets after the boom", Christian-Albrechts-Universität zu Kiel, Germany, September 30-October 2, 2009.

Furthermore, this research was presented under the title COUNTRY AND BORDER EFFECTS IN THE TRANSMISSION OF MAIZE PRICES IN EASTERN AFRICA: EVIDENCE FROM A SEMI-PARAMETRIC REGRESSION MODEL as invited paper at the seminar "Incomplete Pass-Through in Marketing Channels: National and International Trends", Institut D'Économie Industrielle, Université Toulouse 1 Capitole, France, November $16-17$. 


\section{Zusammenfassung}

Diese Arbeit analysiert Faktoren, die Einfluß auf das Ausmaß der inländischen und der grenzübergreifenden Integration der Maismärkte Kenias, Tansanias und Ugandas ausüben. Die Stärke der Reaktion der Preise auf Abweichungen von ihren Langzeitgleichgewichtswerten als Maß der Marktintegration und die Determinanten dieser Reaktion werden für 85 Marktpaare von Januar 2000 bis Oktober 2008 untersucht. Zu diesem Zweck wird ein semi-parametrisches partiell-lineares Regressionsmodell genutzt. Dieses Modell ermöglicht es, den Einfluß der Marktentfernung, der sich als nichtlinear herausstellt, adäquat zu modellieren. Die Ergebnisse der Untersuchung sind kohärent mit der ökonomischen Theorie und von politischem Interesse. Ein Grenzeffekt wird identifiziert, der in dem Sinne heterogen ist, daß er nur für die tansanische, jedoch nicht für die ugandische Grenze festgestellt werden kann. Der tansanische Binnenhandel weißt einen stark negativen Effekt auf, der sich jedoch statistisch nicht vom Grenzeffekt des Landes unterscheidet. Weiterhin wird ein stark positiver Effekt für Nairobi gefunden, der im Einklang mit der Größe und wirtschaftlichen Bedeutung der Stadt im ostafrikanischen Raum steht.

\section{Schlüsselwörter}

Grenzeffekt, Mais, Ostafrika, räumliche Marktintegration, semi-parametrische Regression.

\section{Einleitung}

Mais stellt im subsaharischen Afrika das mit Abstand wichtigste Grundnahrungsmittel dar. Eine solche Rolle hat er auch in Ostafrika inne (AwUOR, 2007), wo er nach FAOSTAT für Tansania und Kenia über ein Drittel und für Uganda mehr als 10\% der durchschnittlichen Kalorienaufnahme ausmacht, weshalb er in Anbau und Handel eine ähnlich wichtige Rolle spielt. Die größten Produzenten der Region sind Tansania und Kenia, wobei ersteres überwiegend selbstversorgend ist, letzteres mit Abstand den größten regionalen Importeur darstellt. Kenias Maisnachfrage wird neben seinen eigenen Anbaugebieten im zentralen Hochland vor allem durch Einfuhren aus Ostuganda und Nordtansania gedeckt, wobei Uganda der größte Nettoexporteur der Region ist (für eine eingehende Behandlung der Maishandelsflüsse siehe AwUOR, 2007 oder Michigan State University, 2008). Nach der UN Comtrade Datenbank gehen im Mittel etwa 60\% der gesamten Maisausfuhren Ugandas und Tansanias nach Kenia, und Mais gehört zu den fünf wichtigsten Ausfuhrgütern in Ostafrika.

Obwohl alle drei Staaten zur Ostafrikanischen Gemeinschaft (East African Community, EAC) gehören, in deren Rahmen seit Januar 2005 eine Zollunion in Kraft ist, verfolgen sie unterschiedliche Agrar- und Handelspolitiken, die nicht immer im Sinne der Union sind. Kenia und Uganda besitzen im Gegensatz zu Tansania überwiegend liberalisierte Märkte. Nach WELTBANK (2008) wurden in allen drei Staaten produktions- und handelsverzerrende Maßnahmen seit 1980 zurückgefahren. Während die ersten beiden Länder ihren Fokus von Besteuerung des Agrarsektors hin zu einer verhaltenen Unterstützung verändert haben, bleibt der Maismarkt in Tansania mit stark politisch 
beeinflußten Preissignalen stark reguliert. Die politischen Entscheidungsträger in Uganda scheinen das Potential des Landes, zur Kornkammer Ostafrikas zu werden und damit auch erhebliche Ausfuhreinnahmen zu realisieren, erkannt zu haben. Daher hat das Land kein Interesse an der Anwendung handelsverzerrender Maßnahmen hat. Die Agrarpolitik Kenias ist eher auf verhaltene Preisstützung und -stabilisierung mittels des National Cereals and Produce Board ausgerichtet, das nach stark zurückgegangener Bedeutung in den letzten 20 Jahren noch begrenzten Einfluß auf die Preise ausübt. Tansania verfolgt in erster Linie das Ziel der flächendeckenden Nahrungsmittelsicherheit, das mit einer Reihe restriktiver Maßnahmen von lokaler bis hin zu nationaler Ebene, wie z.B. Exportverbote, zu erreichen versucht wird (siehe TEMU ET AL. 2007 für einen chronologischen Abriß der Handelspolitik für Mais).

Das Ausmaß, mit dem Preissignale eines Gutes, und damit implizit Aussagen über dessen Angebots- und Nachfragestruktur, zwischen Märkten übertragen werden, ermöglicht Einsichten in den Grad der Marktintegration. Diese läßt ihrerseits Schlußfolgerungen hinsichtlich des Vermögens der Märkte zu, Preis- und dadurch implizierte Wohlfahrtseffekte von Überangebot und Knappheit abzufedern. Eine zentrale Fragestellung besteht in dieser Hinsicht darin, ob die Märkte durch ein langfristiges Preisgleichgewicht miteinander verbunden sind und wie stark sie, falls dieses vorhanden ist, auf Abweichungen davon, die durch abgebots- oder nachfrageinduzierte Schocks ausgelöst werden können, reagieren. Das Ausmaß dieser Reaktion auf zwischenstaatlicher Ebene hängt von verschiedenen Faktoren ab, so z.B. ob die räumlichen Handelsflüsse nationale Grenzen überqueren oder ob bestimmte Länder oder Großstädte sich strukturell vom regionalen Durchschnitt unterscheiden. Daher konzentriert sich diese Arbeit auf die Frage, ob Unterschiede im Ausmaß der Reaktion von Preisen auf Abweichungen von ihren Langzeitgleichgewichtswerten, d.h., nach der Definition von FACKLER und GOODwIN (2001: 978), in der Marktintegration (MI), festgestellt werden können.

Die methodische Analyse besteht aus zwei Schritten. Im ersten Schritt werden 85 Maismarktpaare untersucht, ob sie durch Langzeitgleichgewichte verbunden, d.h. kointegriert sind. Falls dies der Fall ist, wird jeweils ein Vektorfehlerkorrekturmodell (vector error-correction model, VECM) geschätzt. Die ausgeprägte Nettoeinfuhrsituation Kenias und v.a. seiner Hauptstadt Nairobi läßt nicht nur die Untersuchung des Binnenhandels, sondern auch des grenzüberschreitenden Warenverkehrs zwischen Kenia und Tansania bzw. Kenia und Uganda interessant erscheinen. Der Schwerpunkt der Arbeit liegt im zweiten Schritt auf der Analyse von Faktoren, die das Ausmaß der MI beeinflussen. Dies geschieht sowohl auf Grundlage von parametrischen als auch semi-parametrischen Regressionsmodellen. Dabei orientiert sich die Analyse an den einschlägigen Literaturen zu Gravitätsmodellen bzw. Grenzeffekten, die seit den richtungweisenden Veröffentlichungen von MCCALLUM (1995) und ENGEL und ROGERS (1996) Handelsflüsse bzw. Preisvolatilität auf Individual- und Grenzeffekte hin untersuchen, wie z.B. HeLliwell (1996) , Evans (2003) oder Helble (2007). OlPER und RAIMONDI (2008) untersuchen die Bedeutung von Grenzeffekten für den Handel mit Agrargütern.

Die Arbeit ist wie folgt gegliedert. Der nächste Abschnitt erklärt kurz das zugrunde liegende Modell und widmet sich ausführlich der Beziehung zwischen 
Transaktionskosten (TK) und der Stärke der MI und der Rolle von Grenzeffekten als Komponente der TK. Der nachfolgende Abschnitt diskutiert das Problem der Verzerrung von Schätzern als Folge von Fehlspezifikation eines unbekannten funktionalen Zusammenhanges und schlägt alternativ ein semi-parametrisches Regressionsmodell vor, das auch späterhin in der Analyse Anwendung findet. Danach wird kurz auf die Daten eingegangen. In Abschnitt 5 werden die Schätzergebnisse von parametrischen und semi-parametrischen Modellen dargestellt und im Folgeabschnitt ausführlich interpretiert, bevor der siebte Abschnitt den Aufsatz schließt.

\section{Preistransmission, Transaktionskosten und Grenzeffekte}

Nachdem die Zeitreiheneigenschaften der Daten festgestellt wurden, wird mittels des Johansen-Maximum-Likelihood-Schätzers ein VECM je Marktpaar geschätzt, wobei das Hannan-Quinn-Kriterium zur Wahl der Ordnung $k$ der zeitlichen Verzögerungsterme benutzt wird. Ein VECM hat die folgende Form:

$$
\left(\begin{array}{c}
\Delta p_{t}^{A} \\
\Delta p_{t}^{B}
\end{array}\right)=\left(\begin{array}{c}
\alpha^{A} \\
\alpha^{B}
\end{array}\right) e c t_{t-1}+\sum_{i=1}^{k} \boldsymbol{\Gamma}_{i}\left(\begin{array}{c}
\Delta p_{t-i}^{A} \\
\Delta p_{t-i}^{B}
\end{array}\right)+\left(\begin{array}{c}
\varepsilon_{t}^{A} \\
\varepsilon_{t}^{B}
\end{array}\right),
$$

wobei $\Delta p_{t-i}^{l}=p_{t-i}^{l}-p_{t-(i+1)}^{l}, i=0,1, \ldots, k ; l=\{A, B\}$ die (verzögerte) Preisänderung der Märkte $A$ bzw. $B$ darstellt. $\alpha^{l}$ bezeichnet den Ladungs- oder Anpassungsparameter, der das Ausmaß der MI mißt, also die Geschwindigkeit mit der die Preise $p_{t}^{l}$ auf kurzfristige Abweichungen von ihren Langzeitgleichgewichtswerten $p_{t}^{l}$ equ reagieren, die mittels des Fehlerkorrektur-terms $e c t_{t}=p_{t}^{l}-p_{t}^{l \text { equ }}$ quantifiziert werden ${ }^{1}$. $\Gamma_{i}, i=1, \ldots, k$ sind Matrizen der Dimension (2x2), die die Koeffizienten der Kursfristreaktion enthalten, und $\varepsilon_{t}^{l} \sim \operatorname{IIN}\left(0, \sigma_{e}^{2}\right)$ sind Weißes-Rauschen-Fehlerterme. Im zweiten Schritt der Analyse werden, wie im Folgenden dargestellt, die geschätzten Anpassungsgeschwindigkeiten $\hat{\alpha}^{l}$ eingehender untersucht.

Räumlicher Handel ist mit Kosten für die Ausführung der Transaktionen verbunden. In der Literatur werden diese in der Regel in der Eisbergform angenommen, d.h. daß ein Teil des Wertes des Gutes für die Kosten dessen Handels aufgebraucht wird (,abschmilzt“, vgl. z.B. EngEL und Rogers, 1996). Somit erzeugen die Kosten ein Preisintervall, in dem Handel nicht profitabel ist (band of no-arbitrage). Demzufolge reagieren Preise nur auf diejenigen Abweichungen vom Langzeitgleichgewicht, die die jeweiligen TK für Handel zwischen den beiden Märkten $A$ und $B$ (die nicht symmetrisch sein müssen) überschreiten. Die Preisreaktion sinkt somit mit steigenden TK.

Die Literatur zu Grenzeffekten postuliert in der Regel "transportation costs [...] should depend positively on the distance between locations" (ENGEL und RoGERS, 1996).

\footnotetext{
${ }^{1}$ Je höher $\alpha$, desto höher die MI, wenn z.B. $\alpha^{l}=0,5$, dann verändert sich der Preis $p^{l}$ um $50 \%$ des absoluten Ausmaßes des Schocks in der Folgeperiode, es wird also c.p. die Hälfte eines Schocks in einer Periode korrigiert.
} 
Jedoch sind TK im Allgemeinen weder deckungsgleich mit den bloßen Transportkosten, obwohl sie sicherlich einen Großteil derer ausmachen, noch müssen sie zwangsläufig mit der Entfernung ansteigen. Im räumlichen Güterhandel fallen diverse weitere Kosten im Rahmen der physischen Transaktion zwischen Märkten neben denen für den Transport an. BARRETT (2001) diskutiert folgende Komponenten von TK:

$$
\tau^{A B}=f r^{A B}+v^{A B}+z^{A B}+\theta^{A B}
$$

wobei $\tau^{A B}$ die Stück-TK zwischen Märkten $A$ und $B$ und fr die Transportkosten per Einheit darstellen. $v$ beinhaltet variable Kosten, die für Versicherung, Finanzierung, Vertragsschluß etc. anfallen, und $z$ beinhaltet Durchschnittsstückzölle. Schließlich steht $\theta$ für nichtmeßbare TK, wie z.B. Opportunitäts- oder Suchkosten. Die Zerlegung (2) erhebt keinen Anspruch auf Vollständigkeit. Die dort angeführten Teilgrößen werden ihrerseits von verschiedenen Faktoren bestimmt. So werden zum Beispiel die Transportkosten von der Entfernung, dem Zustand der Infrastruktur und der transportierten Menge abhängen, wobei auch die Effizienz des Netzwerkes des Händlers oder beauftragten Spediteurs (wie oft in Entwicklungsländern der Fall) eine Rolle spielen kann. Darüber hinaus betont HelBle (2007) die Bedeutung der geschäftlichen Infrastruktur (business infrastructure), d.h. die Möglichkeit für kurzfristige Geschäftsreisen wird als wesentlicher Faktor identifiziert, da sie den Handel im Durchschnitt signifikant um ein Drittel erhöht.

Es soll an dieser Stelle betont werden, daß diese Komponenten sich allerdings in ihren Effekten auf die TK nicht zwangsläufig verstärken müssen, sondern auch gegenläufigen Beziehungen, bis hin zur gegenseitigen Aufhebung, aufweisen können. So muß z.B. die Beziehung der Höhe der TK zur Entfernung nicht proportional sein. Im Allgemeinen werden TK mit der Entfernung zwar steigen, jedoch können sie für entfernte, durch gute Infrastruktur miteinander verbundene Märkte (z.B. die Hauptstadt und die größte Hafenstadt eines Landes) geringer ausfallen als für nahegelegene, die aber nur durch unbefestigte Wege verbunden sind. In ähnlicher Form wird eine gut entwickelte geschäftliche Infrastruktur zu geringeren TK zwischen entfernten Märkten führen als für nahegelegene, die diese nicht aufweisen.

Die Mehrzahl dieser Komponenten ist in der Praxis schwerlich meßbar. Immerhin können sie aber in Kategorien aufgeteilt werden, die für jeden Markt einfach festzustellen sind. Die Entfernung stellt die einzige Variable dar, die kontinuierlich und außerdem einfach meßbar ist. Da sie einen Großteil der Frachtkosten ausmacht, sollte sich dies auch in den Daten widerspiegeln, d.h. ein signifikanter Entfernungseffekt (distance effect) sollte nachweisbar sein. Im Falle grenzüberschreitenden Handels können weitere Kosten außer denen für die Fracht anfallen, wie z.B. Einfuhrzölle, Ausfuhrsteuern, Kosten für die Beschaffung der notwendigen Einfuhrpapiere oder wegen langer Wartezeiten. Diese können einen Grenzeffekt (border effect) hervorrufen, d.h. also einen signifikanten Unterschied im Ausmaß der MI zwischen inländischem und grenzüberschreitendem Handel. Schließlich erscheint es plausibel, daß Komponenten der TK, wie z.B. die Qualität der Infrastruktur, Besteuerung oder Subventionierung von Treibstoff oder handelsrelevante Institutionen, länderspezifisch sein. 
Somit kann potentiell auch ein Landes-(Individual-)effekt eine relevante Komponente von TK ausmachen.

TK können demnach als eine Funktion der Entfernung und von Grenz- und Landeseffekten modelliert werden. Letztere werden durch Platzhaltervariablen modelliert, die den Wert 1 annehmen, wenn das Charakteristikum der Kategorie erfüllt ist und sonst $0^{2}$. Im Speziellen werden Platzhaltervariablen für einen allgemeinen Grenzeffekt $\left(D_{G}\right)$ berücksichtigt, der aber aufgrund der oben beschriebenen heterogenen Politikansätze in einem alternativen Modell in einen Effekt der kenianisch-tansanischen Grenze $\left(D_{K T}\right)$ und der kenianisch-ugandischen $\left(D_{K U}\right)$ Grenze unterschieden wird. Weiterhin werden aus dem gleichen Grund Platzhalter für Landeseffekte von Tansania $\left(D_{T a n}\right)$ und Uganda $\left(D_{U g}\right)$ einbezogen, um mögliche Heterogenität hinsichtlich der MI abbilden zu können ${ }^{3}$. Schließlich findet auch ein Platzhalter für Markpaare, einer deren Märkte Nairobi ist, Berücksichtigung $\left(D_{N a i}\right)$, da es um die größte und wirtschaftlich bedeutendste Großstadt in Ostafrika handelt, und somit durchaus eine besondere Rolle hinsichtlich der Maisnachfrage und des Handels spielen kann.

Da Einflußfaktoren des Ausmaßes der Preisreaktion auf Abweichungen vom Langzeitgleichgewicht im Zentrum dieser Arbeit stehen, erscheint die Summe der absoluten geschätzten Anpassungsgeschwindigkeiten pro Marktpaar $S_{\alpha}^{A B}=\left|\alpha^{A}\right|+\left|\alpha^{B}\right|$ (die paarweise Anpassung) als die relevante Variable, deren Determinanten im zweiten Schritt der Analyse untersucht werden ${ }^{4}$. Dies ist gerechtfertigt, da das Ausmaß der MI von der Reaktion beider Preise auf Abweichungen vom Gleichgewicht abhängt ${ }^{5}$. Somit ergibt sich die folgende Gleichung:

$$
S_{\alpha}^{A B}=f\left(d^{A B}\right)+\beta_{0}+\beta_{1} D_{G}^{A B}+\beta_{2} D_{T a n}^{A B}+\beta_{3} D_{U g}^{A B}+\beta_{4} D_{N a i}^{A B}
$$

bzw. wird einer weiteren Spezifikation in heterogene Grenzeffekte unterschieden und $D_{G}^{A B}$ durch $D_{K T}^{A B}$ und $D_{K U}^{A B}$ ersetzt.

Vor dem Hintergrund der stark handelsverzerrenden tansanischen Politik erscheint ein Grenzeffekt hinsichtlich der paarweisen Anpassung für denkbar, der demnach negativ ausfallen sollte, was implizieren würde, daß trotz Zollunion noch erhebliche TKerhöhende Faktoren für diesen grenzüberschreitenden Handel existieren. Weiterhin wird signifikanter Einfluß der Entfernung erwartet, wie es auch plausibel scheint, aufgrund der Heterogenität der drei Staaten Landeseffekte zu finden, wobei wiederum Tansania eine besondere Rolle spielen könnte und mit einiger Sicherheit einen

\footnotetext{
${ }^{2}$ Die Einbeziehung solcher Variablen mag auf den ersten Blick sehr grob erscheinen, jedoch ist diese Herangehensweise die einzig praktikable vor dem Hintergrund des Problems der Identifizierung und Quantifizierung der Komponenten der TK.

${ }^{3}$ Die Platzhalter für die Grenzeffekte bzw. die Landeseffekte nehmen den Wert 1 an, wenn der Handel zwischen den Märkten des Paares die jeweilige Grenze überschreitet bzw. innerhalb des jeweiligen Landes stattfindet.

${ }^{4}$ Dieses Maß impliziert eine Preistransmissionselastizität von $\beta_{1}=1$ in $e c t_{t}=p_{t}^{A}-\beta_{0}-\beta_{1} p_{t}^{A}$.

${ }^{5}$ In der Summe werden nur Koeffizienten berücksichtigt, die signifikant zum $10 \%$ Niveau geschätzt wurden.
} 
negativen Effekt (d.h. geringere MI) aufweisen wird. Auch erscheint eine Sonderrolle Nairobis, begründet durch seine herausragende Bedeutung in der EAC, denkbar.

\section{Unbekannte Funktionsform und das partiell-lineare Regressionsmodell}

In Gleichung (3) ist die paarweise Anpassung $S_{\alpha}^{A B}$ unter anderem eine Funktion der Entfernung $d^{A B}$ zwischen zwei Märkten. Jedoch kann die ökonomische Theorie keine eindeutigen Hinweise auf die explizite Form dieser partiellen funktionalen Beziehung geben außer der allgemeinen Aussage, dass sie tendenziell mit der Entfernung abnehmen sollte. Aus der Ökonometrie ist bekannt, daß eine Fehlspezifikation einer funktionalen Beziehung zu ernstzunehmenden Verzerrungen der Schätzer führt. Teilweise versucht die Literatur zu Grenzeffekten dieser Herausforderung in begrenztem Umfang zu begegnen, indem alternative Funktionsformen geschätzt werden. Engel und Rogers (1996) sind sich dieses Problems bewußt, indem sie anmerken: „The effect of distance may also be understated if the log-distance function is not the appropriate one." und sowohl eine logarithmische als auch eine quadratische Spezifikation schätzen.

In dieser Arbeit wird ein davon abweichender Ansatz gewählt, indem keine (potentiell zu restriktive und damit wahrscheinlich unzutreffende) Annahme über die funktionale Form gemacht wird. Der partielle Einfluß der Entfernung wird nicht in ein „parametrisches Korsett“ gezwängt, sondern mittels einer semi-parametrischen Version des Modells (3) geschätzt (HÄRDLE ET AL. 2004). Im Speziellen scheint ein partielllineares Modell geeignet, das es erlaubt, den partiellen Einfluß der Entfernung, der im gegebenen Kontext durchaus nichtlinear sein könnte, nichtparametrisch zu schätzen. Dies vermeidet die Gefahr, eine falsche Funktionsform anzunehmen, jedoch erlaubt gleichzeitig den linearen partiellen Einfluß der Platzhaltervariablen zu berücksichtigen. Es vereint die Vorzüge der vollkommenen Flexibilität eines nichtlinearen Regressionsmodells und der intuitiven Interpretierbarkeit einer simplen multivariaten Regression. Somit wird folgende semi-parametrische Version von Modell (3) geschätzt:

$$
S_{\alpha}^{A B}=m\left(d^{A B}\right)+\beta_{1} D_{G}^{A B}+\beta_{2} D_{\text {Tan }}^{A B}+\beta_{3} D_{U g}^{A B}+\beta_{4} D_{\text {Nai }}^{A B},
$$

die in einer zweiten Version wiederum in heterogene Grenzeffekte differenziert. Die einzige Annahme über die funktionale Beziehung $m(\bullet)$ besteht darin, daß es sich um eine glatte Funktion handelt, die auch die Konstante $\beta_{0}$ aus Modell (3) aufnimmt. Sie wird ebenso wie die Koeffizienten der Platzhaltervariablen als partieller Einfluß der Entfernung zwischen den beiden Märkten eines Paares auf die paarweise Anpassung des Paares interpretiert, jedoch mit dem Unterschied, daß ihre Form vollkommen flexibel anhand der Daten ermittelt wird. Die Annahme der Glätte der funktionalen Beziehung ist offensichtlich weit weniger einschränkend als die einer expliziten parametrischen Spezifikation. Außerdem ermöglicht ein solcher let the data speakAnsatz neben Einsichten bezüglich der wahren, potentiell nichtlinearen Beziehung auch ökonometrische Tests hinsichtlich der Approximierbarkeit der nichtparametrischen durch parametrische Funktionen. 


\section{Datengrundlage}

Es wird ein umfangreicher Datensatz untersucht, der aus 16 Zeitreihen von Großhandelsmaispreisen in Kenia (Eldoret Nairobi, Nakuru, Mombasa), Tansania (Aruscha, Daressalam, Iringa, Mbeya, Songea) und Uganda (Iganga, Kampala, Kasese, Lira, Mbale, Masaka, Masindi) besteht ${ }^{6}$. Er wurde aus mehreren Quellen zusammengestellt, welche das Ostafrikanische Handelsnetzwerk Ratin (www.ratin.net/), den Bericht der MichigAN STATE UnIVERSITY (2008), das tansanische Industrie- und Handelsministerium (www.mitm.go.tz/) und InfoTradeUganda (www.infotradeuganda.com/) umfassen. Die Entfernungen wurden in Google.maps (http://maps.google.de/) als kürzeste Entfernung im Überlandstraßennetz der Länder ermittelt. Jede Zeitreihe umfaßt 106 monatliche Beobachtungen von Januar 2000 bis Oktober 2008. Fehlende Beobachtungen (59 von 1696, d.h. 3,5\%) wurden mittels einer Version des von KING ET AL. (2001) publizierten Algorithmus berechnet, indem 1000 Werte pro Datenpunkt erzeugt wurden, aus denen mithilfe des nichtparametrischen Modusschätzers von Parzen der wahrscheinlichste Wert ermittelt wurde.

Alle Preise wurden in US\$/t umgerechnet und in logarithmierter Form verwendet. Alle Zeitreihen sind nach dem ADF-Test integriert in erster Ordnung (I(1)), d.h. besitzen eine Einheitswurzel zum $10 \%$ Signifikanzniveau ${ }^{7}$. Unter allen möglichen Kombinationen werden 85 berücksichtigt, die sämtliche inländische Marktpaare in Kenia (6), Tansania (10) und Uganda (21) sowie, aufgrund der in der Einführung beschriebenen Handelsflüsse, sämtliche Kombinationen zwischen Kenia und Tansania (20) bzw. Kenia und Uganda (28) umfassen.

\section{Abbildung 1: Paarweise Anpassung vs. Entfernung}

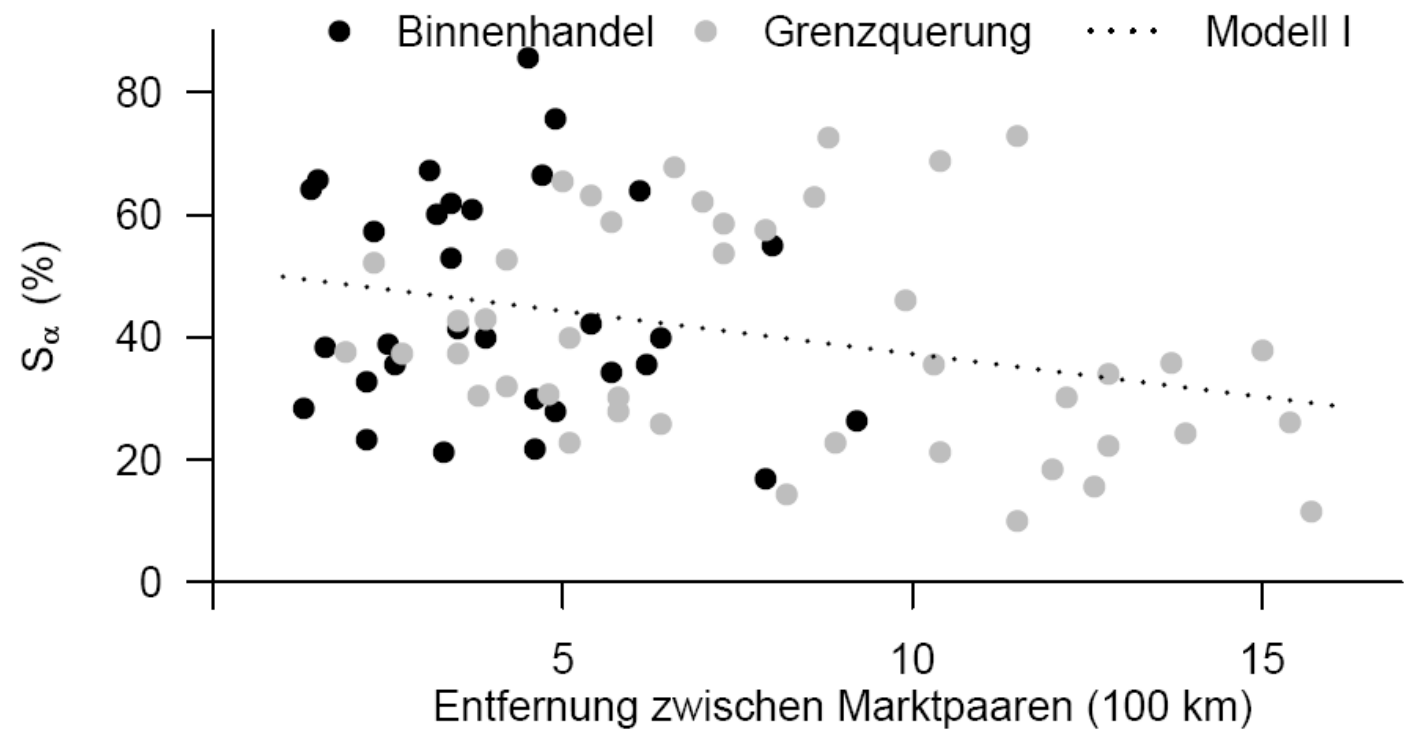

Quelle: Eigene Darstellung.

\footnotetext{
${ }^{6}$ Für die geografische Verteilung dieser Märkte siehe Abbildungen im Anhang.

7 Aufgrund der formalen Einschränkungen können weder die Ergebnisse dieser Tests noch die Schätzergebnisse des VECM (1) hier ausführlich wiedergegeben werden, sind aber auf Nachfrage von den Autoren erhältlich.
} 
Für die 77 Paare, die nach dem Johansen-Spur-Test kointegriert auf dem 10\% Niveau sind, wird jeweils das VECM (1) geschätzt. Drei Paare darunter weisen falsche Vorzeichen der Anpassungsparameter auf, so daß sie als Ausreißer von der weiteren Analyse ausgeschlossen werden. Somit werden 74 Marktpaare im zweiten Schritt der Analyse verwendet (Abbildung 1), deren paarweise Anpassung $S_{\alpha}^{A B}$ minimal 10,1\%, im Mittel 42,2\% und maximal 85,7\% beträgt. Die Entfernung zwischen den Marktpaaren beträgt minimal $130 \mathrm{~km}$, im Mittel $650 \mathrm{~km}$ und maximal $1770 \mathrm{~km}$ und geht in Einheiten von $100 \mathrm{~km}$ in die Regression ein. 58\% der Paare beinhalten eine Grenzüberquerung, darunter 20\% die kenianisch-tansanische und 38\% die kenianischugandische. 8\% der Paare befinden sich vollständig in Tansania und 26\% in Uganda. 19\% der Paare beinhalten Nairobi.

\section{Schätzergebnisse}

\subsection{Parametrische Modelle}

In Abschnitt 2 wurde eine Reihe von Variablen hergeleitet, die potentiell einen Einfluß auf die paarweise Anpassung $S_{\alpha}^{A B}$ haben können, der zuerst mit verschiedenen Spezifikationen des parametrischen Modells (3) untersucht wird. Jedoch ist es aus der ökonomische Theorie nicht möglich, für den Einzelfall die relevanten Variablen zu bestimmen, die $S_{\alpha}^{A B}$ am besten erklären. Die Variablenauswahl wird daher durch eine statistische Modellauswahl auf Basis Akaikes Informationskriteriums (AIC) ergänzt, um diejenigen Variablen zu identifizieren, die über die größte Erklärungskraft hinsichtlich $S_{\alpha}^{A B}$ verfügen. Dasjenige Modell gilt in diesem Sinne als das „beste“, das die Daten mit so wenigen wie möglich, aber so vielen wie nötig, also nur den aussagekräftigsten Variablen erklärt. Das AIC wird allgemein berechnet als:

(5) $\quad A I C=-2 \ln (L)+2 k$

wobei $L$ die Mutmaßlichkeit (likelihood) des Modells und $k$ die Anzahl der geschätzten Parameter benennt. Je besser die Daten durch das Modell erklärt werden, umso höher wird $L$ und umso geringer wird der erste Teilterm von (6). Da sich dies einfach durch Erhöhung der Anzahl erklärender Variablen erreichen läßt, sorgt der so genannte Strafterm $2 k$ für einen Ausgleich, indem er Variablen mit geringem Erklärungsgehalt „bestraft“ und das AIC dementsprechend erhöht. Daher ist das im statistischen Sinne beste Modell dasjenige, das das kleinste AIC aufweist, wie in Abbildung 2 dargestellt. 
Abbildung 2: AIC für verschiedene Spezifikationen von (4)

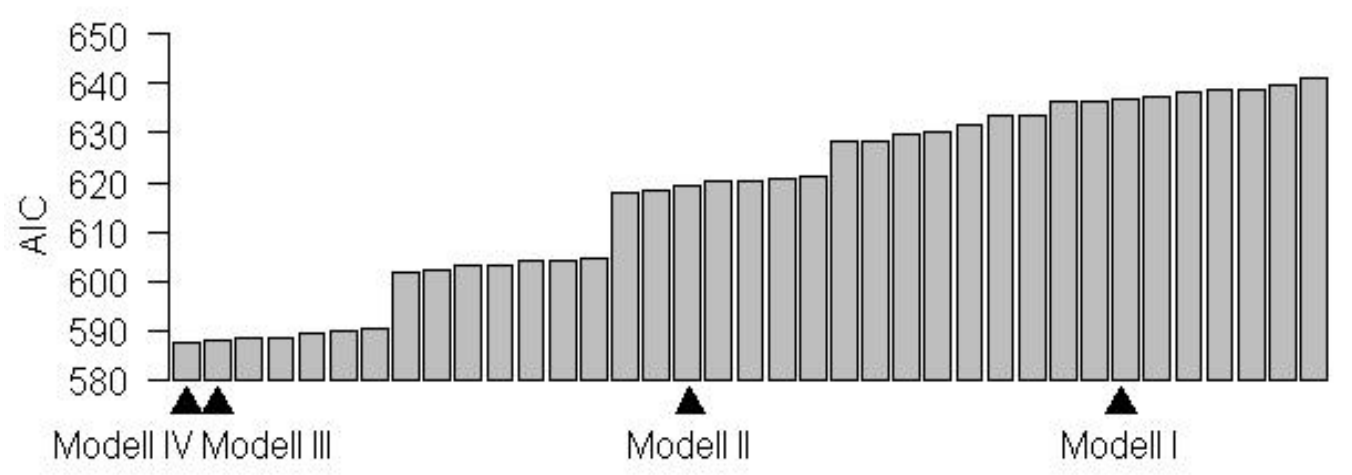

Modellvarianten

Quelle: Eigene Darstellung.

Tabelle 1 gibt die Ergebnisse auf Basis des Kleinsten-Quadrat-Schätzers, die Signifikanzen und das AIC einiger ausgewählter Modelle wieder. In Abbildung 1 läßt die gepunktete Linie eine funktionale Beziehung zwischen der Entfernung und $S_{\alpha}^{A B}$ vermuten, was durch die Schätzergebnisse von Modell I als signifikant negativer Zusammenhang bestätigt wird. Modell II zeigt, daß ein allgemeiner Grenzeffekt $\left(D_{B}\right)$ nicht existiert, aber ein Nairobieffekt $\left(D_{N a i}\right)$ signifikant positiv ist, was zu einem besseren Modell führt. Wenn jedoch, wie in Modell III, der Effekt der kenianischtansanischen Grenze $\left(D_{K T}\right)$ und ein Ländereffekt für Tansania $\left(D_{T a n}\right)$ berücksichtigt werden, was das AIC um mehr als 40 Punkte reduziert (für Uganda ist weder ein Grenz- noch ein Landeseffekt signifikant), ist der partielle Einfluß der Entfernung nicht mehr signifikant ${ }^{8}$, womit diese Variable überflüssig wird.

Abbildung 3: AIC für verschiedene Bandbreiten des semi-parametrischen Modells

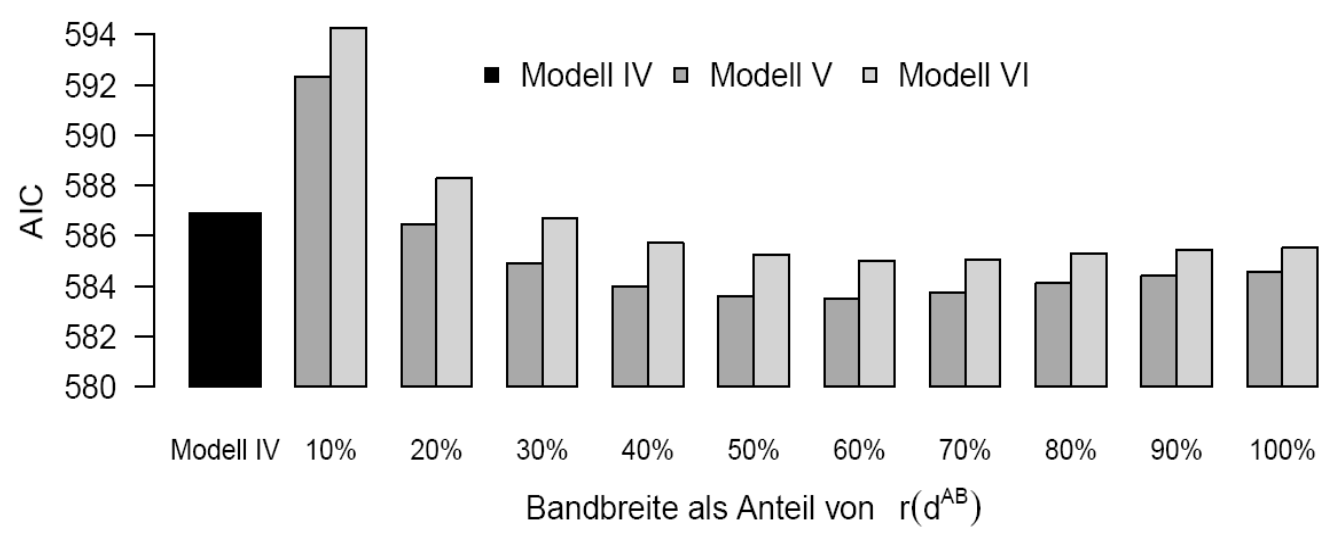

Quelle: Eigene Darstellung.

Die Koeffizienten des tansanischen Grenz- $\left(D_{K T}\right)$ und Ländereffektes $\left(D_{T a n}\right)$ sind beide signifikant negativ und von ähnlicher Größe. Daher wird mittels eines Waldtests geprüft, ob beide Koeffizienten statistisch voneinander abweichen, d.h. ob beide

\footnotetext{
${ }^{8}$ Eine lineare oder logarithmische Modellierung dieses partiellen Einflusses sind ebenso nicht signifikant.
} 
Variablen zu einem allgemeinen Tansaniaplatzhalter $D_{\text {TanAll }}=D_{\text {Tan }}+D_{K T}$ zusammengefaßt werden können. Die entsprechende Teststatistik ist mit einem Wert von 1,4 (p-Wert 0,25) nicht signifikant ${ }^{9}$. Somit kann das Modell vereinfacht werden, was sich im leicht verbesserten AIC von 586,9 widerspiegelt ${ }^{10}$. Somit repräsentiert Modell IV das beste parametrische Modell.

Tabelle 1: Schätzergebnisse für verschieden Spezifikationen von Modell (4) ${ }^{a}$

\begin{tabular}{|c|c|c|c|c|c|c|c|c|}
\hline \multirow[b]{2}{*}{ Modell } & \multicolumn{8}{|l|}{ Variable } \\
\hline & Kostante & d & $d^{2}$ & $D_{K T}$ & $\boldsymbol{D}_{\text {Tan }}$ & $D_{\text {Nai }}$ & $D_{\text {TanAII }}$ & AIC \\
\hline I & $51,3 * * *$ & $-1,4 * * *$ & & & & & & 637,0 \\
\hline II & $49,4 * * *$ & $-1,3^{* *}$ & & & & $16,9 * * *$ & & 629,6 \\
\hline III & $42,2 * *$ & 2,0 & $-0,2$ & $-26,0 * * *$ & $-22,7 * * *$ & $15,2^{* * *}$ & & 587,9 \\
\hline IV & $46,9 * * *$ & & & & & $15,2 * * *$ & $-26,5 * * *$ & 586,9 \\
\hline
\end{tabular}

Quelle: Eigene Berechnung.

${ }^{a}$ Ein, zwei und drei Sternchen markieren Signifikanz zum 10\%, 5\% bzw. 1\%-Niveau.

\subsection{Semi-parametrische Modelle}

Im besten parametrischen Modell IV konnte kein signifikanter partieller Einfluß der Entfernung auf die paarweise Anpassung nachgewiesen werden. Diese Variable wird im Folgenden auf möglichen nichtlinearen Einfluß untersucht. Es werden mittels des Speckmanschätzers (HÄRDLE ET AL., 2004) die beiden partiell-linearen Modelle $S_{\alpha}^{A B}=m\left(d^{A B}\right)+\beta_{1} D_{\text {Tan }}^{A B}+\beta_{2} D_{K T}^{A B}+\beta_{3} D_{\text {Nai }}^{A B} \quad$ (Modell V) bzW. $S_{\alpha}^{A B}=m\left(d^{A B}\right)+\gamma_{1} D_{\text {TanAll }}^{A B}+\gamma_{2} D_{\text {Nai }}^{A B}$ (Modell VI) geschätzt, die in ihrer Variablenauswahl den parametrischen Modellen III bzw. IV in Tabelle 1 entsprechen. Die Schätzung wird für verschiedene Bandbreiten $h$, die die Glätte des nichtparametrischen Teils $m\left(d^{A B}\right)$ bestimmen ${ }^{11}$, zur Kontrolle der Robustheit der Ergebnisse durchgeführt (Abbildung 4). Abbildung 3 verdeutlicht, daß das semi-parametrische Modell für die meisten Bandbreiten ein geringeres AIC als das beste parametrische Modell $(586,9)$ aufweist. Damit wird gezeigt, daß eine Berücksichtigung der Entfernung in Form eines nichtlinearen partiellen Einflusses das Modell signifikant verbessert. Das AIC erscheint für beide Modellvarianten recht stabil über ein weites Spektrum von Bandbreiten, was darauf hinweist, daß die Verbesserung der Modellqualität recht robust ist. Model VI weist ein geringeres AIC als Variante V auf.

Das Minimum liegt bei einer Bandbreite von 60\% der Spannweite $r\left(d^{A B}\right)$, d.h. $h_{o p t}=$ 8,6. Das entsprechende AIC ist mit einem Wert von 583,5 über 3 Punkte geringer als das von Modell VI, was auf eine beachtliche Verbesserung des Modells durch die

\footnotetext{
${ }^{9}$ Ein ähnlicher Test auf Parametergleichheit des Nairobi- und des allgemeinen Tansaniaplatzhalters wird mit einer Teststatistik von 73,4 (p-Wert <0,001) eindeutig abgelehnt.

${ }^{10}$ Somit wird ersichtlich, daß eine Verbesserung des AIC äquivalent zu den Ergebnissen des Waldtests ist und auf ein signifikant besseres Modell hinweist.

${ }^{11}$ Als Bandbreiten werden die Dezile der Spannweite der Entfernung $r\left(d^{A B}\right)=\max \left(d^{A B}\right)-\min \left(d^{A B}\right)=14.4$ benutzt. Je höher die Bandbreite, umso stärker wird der nichtparametrische Schätzer geglättet.
} 
nichtparametrische Modellierung des partiellen Einflusses der Entfernung hinweist. Das optimale semi-parametrische Modell ist demnach:

$$
S_{\alpha}^{A B}=m\left(d^{A B}\right)-24,9 * * * \cdot D_{\text {TanGen }}^{A B}+15,2 * * * \cdot D_{\text {Nai }}^{A B}+\varepsilon^{A B} .
$$

Die Schätzwerte des parametrischen Teils sind denen von Modell IV sehr ähnlich, wobei ihre Schätzer frei von ökonometrischer Verzerrung aufgrund falscher Funktionsform sind. Ein signifikant negativer allgemeiner Tansaniaeffekt von etwa 25 Prozentpunkten und ein ähnlich signifikant starker, aber positiver Nairobieffekt von etwa 15 Prozentpunkten werden identifiziert.

In Abbildung 4 ist der geschätzte nichtparametrische Einfluß $m\left(d^{A B}\right)$ für verschiedene Bandbreiten dargestellt. Der partielle Einfluß der Entfernung bedeutet für nahegelegene Märkte eine mittlere paarweise Anpassung von etwa 47\%, d.h. 47\% der Abweichung vom Langfristgleichgewicht wird vom Marktpaar unmittelbar in der Folgeperiode korrigiert. Bis zu einer Entfernung von 500 bis 700 km steigt sie leicht auf 48\% an und fällt danach mit steigender Distanz. Jenseits von etwa $1000 \mathrm{~km}$ fällt sie mit konstanter Veränderungsrate von einem Prozentpunkt pro $100 \mathrm{~km}$ zusätzlicher Entfernung ${ }^{12}$. Somit zeigen Marktpaare, deren Märkte $1500 \mathrm{~km}$ voneinander entfernt liegen, eine mittlere paarweise Anpassung von nur noch 42\%.

\section{Abbildung 4: Geschätzter nichtlinearer Einfluß der Entfernung auf die paarweise Anpassung}

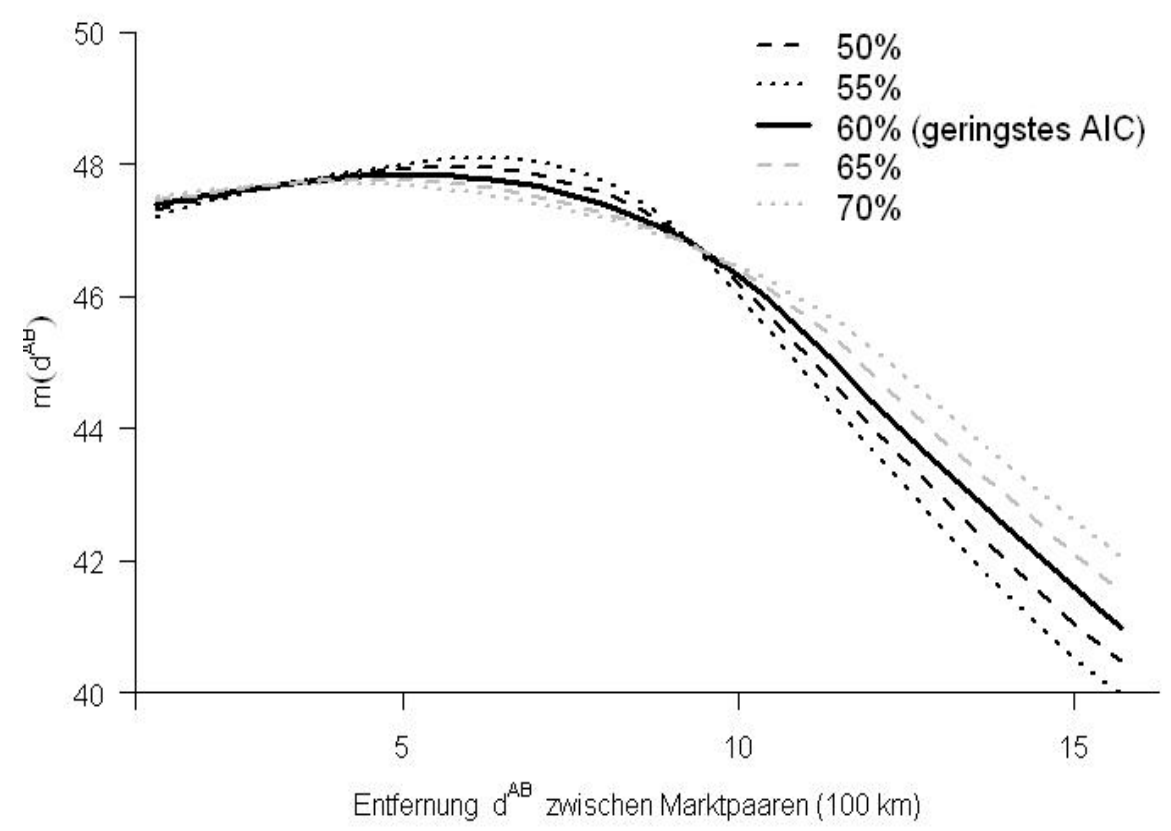

Quelle: Eigene Darstellung.

${ }^{12}$ Abbildung 4 im Anhang stellt die geschätzte nichtlineare Funktion für die optimale Bandbreite dar. 
In Abbildung 5 ist $m\left(d^{A B}\right)$ im Vergleich zu verschiedenen parametrisch modellierten partiellen Einflüssen abgebildet. Schon bei visueller Inspektion fällt auf, daß letztere stark vom nichtlinearen Schätzer abweichen, was durch die Ergebnisse eines modifizierten Bootstrap-Likelihood-Ratio-Tests (HÄRDLE ET AL., 2004) für 1000 Wiederholungen und $h_{o p t}=8,6$ bestätigt wird. Die Nullhypothesen, daß die partielle Beziehung zwischen $d^{A B}$ und $S_{\alpha}^{A B}$ linear $\left(m\left(d^{A B}\right)=\beta_{0}+\beta_{1} d^{A B}\right)$, quadratisch $\left(m\left(d^{A B}\right)=\beta_{0}+\beta_{1} d^{A B}+\beta_{2}\left(d^{A B}\right)^{2}\right)$ bzw. logarithmisch $\left(m\left(d^{A B}\right)=\beta_{0}+\beta_{1} \log \left(d^{A B}\right)\right)$ ist, werden jeweils mit, zum 1\%-Niveau signifikanten, Teststatistiken von 29,3, 5,4 bzw. 76,5 zugunsten der Alternativhypothese eines nichtlinearen Einflusses abgelehnt. Damit besteht starke ökonometrische Evidenz, daß eine parametrische Modellierung des partiellen Einflusses für die hier analysierten Daten inadäquat wäre, was konsistent mit der Modellauswahl auf Basis des AIC ist.

\section{Abbildung 5: Nichtparametrische vs. parametrische partielle Einflüsse}

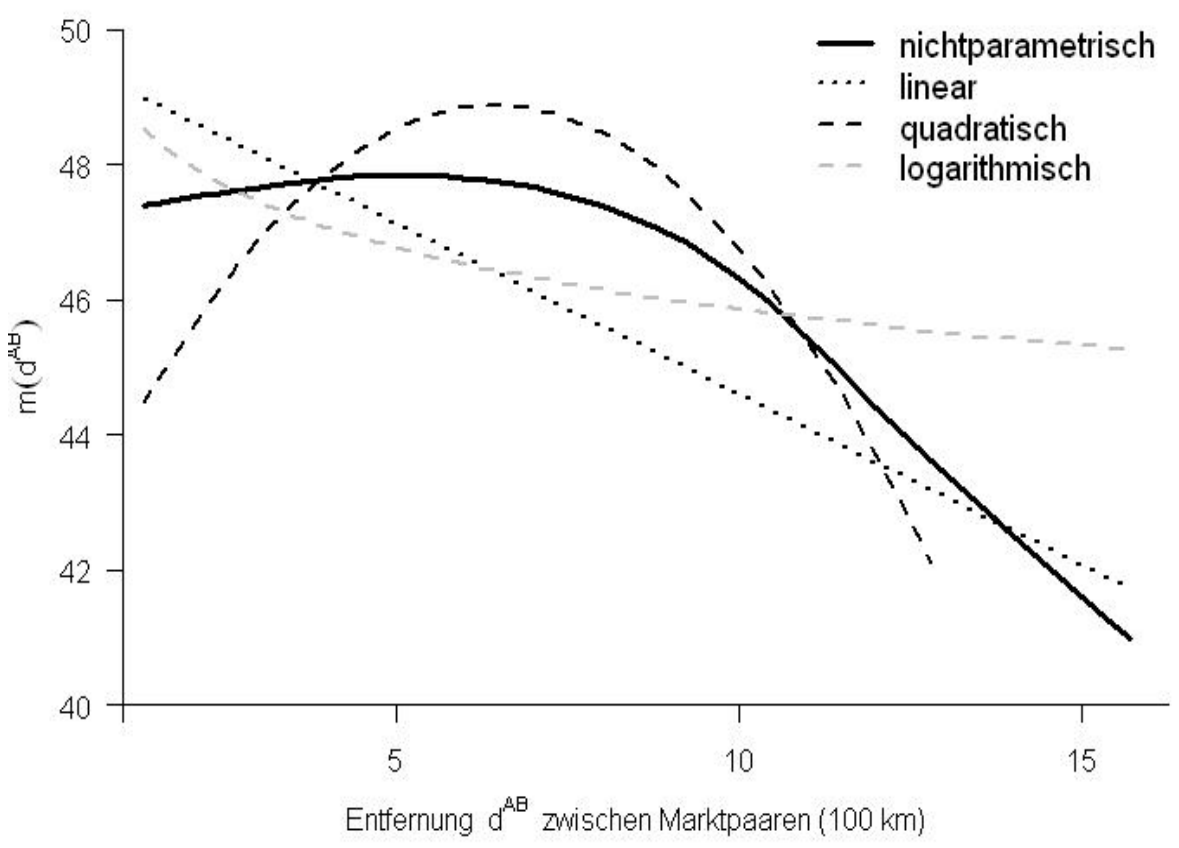

Quelle: Eigene Darstellung.

\section{Diskussion der Schätzergebnisse}

Beide Modelle weisen einen signifikant negativen Effekt sowohl für tansanische Inlandsmärkte als auch für die tansanische Grenzquerung nach, was konsistent mit der vom diesem Land verfolgten Agrar- und Handelspolitik, wie in der Einleitung beschrieben, ist. Dieser Effekt verringert, c.p., die mittlere paarweise Anpassung um etwa 25 Prozentpunkte und kann somit als außerordentlich starker Effekt angesehen werden. Obwohl der Punktschätzer des isolierten kenianisch-tansanischen Grenzeffektes, plausible Folge der häufig angewandten Ausfuhrverbote, absolut größer als der des isolierten tansanischen Landeseffektes, bedingt durch handelsverzerrende 
Maßnahmen auf lokaler und regionaler Ebene, ist, unterscheidet er sich statistisch nicht von letzterem. Somit geht der tansanische Grenzeffekt in einem allgemeinen Tansaniaeffekt auf, was zeigt, daß sämtliche Marktpaare, die mindestens einen tansanischen Markt beinhalten, signifikant schwächer integriert sind. Dies impliziert auf Basis der im zweiten Abschnitt entwickelten Gedanken, daß die TK im Land und an dessen Grenzen signifikant höher sind als in bzw. zwischen Kenia und Uganda. Einen wesentlichen Grund stellen mit Sicherheit die stark preis- und handelsverzerrenden Maßnahmen der tansanischen Regierung zur Sicherstellung flächendeckender Nahrungsmittelsicherheit dar. Die Identifizierung weiterer tansaniaspezifischer Faktoren liegt jenseits des Rahmens dieser Analyse, jedoch erscheint sie von erheblichem Interesse, da sie zu politikorientierten Handlungsempfehlungen zur Reduzierung bestehender TK führen könnte,.

Ein allgemeiner Grenzeffekt konnte nicht gefunden werden. Es wurde aber festgestellt, daß die Unterscheidung in einen kenianisch-tansanischen und kenianisch-ugandischen Effekt notwendig ist, was vor dem Hintergrund der stark differierenden nationalen Politiken als recht nahe liegend scheint. Während die Querung ersterer Grenze die MI signifikant verringert, liegt kein Effekt im Falle letzterer vor. Das deutet darauf hin, daß ein Grenzeffekt nicht zwangsläufig als homogen für ein Land aufgefaßt werden muß, sondern es sich, wie hier aus der Sicht Kenias ${ }^{13}$, um einen heterogenen Effekt handeln kann, der, unseres Wissens nach, in der Literatur zu Grenzeffekten noch nicht in dieser Form berücksichtigt worden ist. Weitere Untersuchungen über die Gründe des Auftretens solcher Heterogenität sind relevant von einem praktischen Standpunkt, liegen aber jenseits des Rahmens dieses Aufsatzes.

Neben dem Tansaniaeffekt wird ein signifikant positiver Nairobieffekt in vergleichbarer Stärke nachgewiesen, d.h. die Integration von Nairobi mit anderen Märkten ist im Mittel um 15 Prozentpunkte höher als für Marktpaare ohne die Stadt. Die Lage des Partnermarktes, ob in Kenia oder in einem der Nachbarländer, ist dabei nicht ausschlaggebend. Dieses Ergebnis ist vor dem Hintergrund der Größe und der wirtschaftlichen Rolle der Stadt im ostafrikanischen Raum und der recht liberalen Handelspolitik Kenias sehr plausibel. Da sie die Hauptstadt und die bei weitem größte Stadt des Landes darstellt, besitzt sie eine überdurchschnittlich gute infrastrukturelle Anbindung nicht nur an den Rest des Landes, sondern v.a. auch an die Nettoausfuhrregionen für Mais der Nachbarländer. Weiterhin dürfte die Attraktivität der Stadt als Absatzmarkt für Grundnahrungsmittel aufgrund der Zahl und des Wohlstandes ihrer Bewohner einen weiteren Faktor darstellen, wo während es ganzen Jahres der Verkauf größerer Mengen, ggf. auch zu höheren Preisen im Gegensatz zu kleineren Städten erwartet werden kann. Spediteure können häufigen Verkehr von Gütern und Personen zu und von der Stadt erwarten, so daß weniger Leerfahrten anfallen, was einerseits Kosten verringert und andererseits den Wettbewerb erhöht, wodurch Skaleneffekte und spill-overs entstehen können. Nicht zuletzt wird die erwähnte geschäftliche Infrastruktur, wie von HELBLE (2007) für Europa nachgewiesen, im Falle Nairobis, v.a. wegen dessen wirtschaftlicher Bedeutung und dem überdurchschnittlichen Zustand der den Handel betreffenden Infrastruktur eine erhebliche Rolle spielen. Es scheint plausibel, daß die Stadt mit

\footnotetext{
${ }^{13}$ Die spezielle Relevanz solcher Heterogenität des Effektes für Entwickungsländer scheint plausibel.
} 
weniger Mühe und Kosten erreicht werden kann als andere, die ebenso weit entfernt von den Produzentenregionen liegen. Solche und andere Faktoren verringern die Kosten räumlichen Handels im Falle Nairobis in einem Umfang, daß schon sehr geringe Preisanreize mit sehr geringer Verzögerung beachtliche Handelsströme auslösen können. Daher sind die entsprechenden Marktpaare durch überdurchschnittlich starke paarweise Anpassung, d.h. überdurchschnittliche MI, gekennzeichnet.

Im Kontext der soeben behandelten Komplexe eines Nairobi-, eines Landes- und eines Grenzeffektes liegt eine weitere Erklärung nahe. Im Falle Nairobis, dem Schwergewicht in der EAC, können auf Basis von Verwaltungseinheiten definierte Grenzen wesentlich weniger ausschlaggebend sein als auf Basis von ökonomischen Märkten definierte. Obwohl z.B. Handel von einem Markt eines Nachbarlandes nach Nairobi administrative Grenzen überschreiten muß, ist die paarweise Anpassung um 15 Prozentpunkte höher als für ein gleichentferntes Paar ohne Nairobi, gleichgültig in welchen Ländern, da das erste Marktpaar innerhalb einer „ökonomischen Einheit“ im Gegensatz zum zweiten liegt. Diese Sichtweise wird durch den stark negativen Landeseffekt Tansanias gestützt. Das Land scheint aufgrund von auf lokaler und regionaler Ebene angewandter handelsverzerrender Maßnahmen in mehrere ökonomische Einheiten zersplittert zu sein, obwohl es eine administrative Einheit darstellt.

Sowohl das parametrische als auch das semi-parametrische Modell geben hinsichtlich der partiellen Einflüsse der soeben behandelten kategorialen Variablen sehr ähnliche Ergebnisse, doch unterscheiden sie sich stark im Hinblick auf die Rolle der Entfernung. Im ersten Modell erscheint sie weder in linearer, quadratischer noch logarithmischer Form signifikant. Im zweiten Modell verbessert sie, wenn in nichtlinearer Form berücksichtigt, jedoch die Güte des Modells erheblich. Wie sind diese scheinbar widersprechenden Ergebnisse miteinander vereinbar? In Modell I, in dem Entfernung als die einzig relevante Variable angenommen wird, hat sie einen signifikant negativen Einfluß auf die paarweise Anpassung, jedoch ist eine solche Spezifikation weit entfernt vom optimalen Modell, in dem Tansania einen stark negativen und Nairobi einen stark positiven Effekt hat und die Entfernung insignifikant wird. Das erscheint plausibel, wenn man die Information, die in den beiden Effekten beinhaltet ist, berücksichtigt. Beide Platzhaltervariablen beinhalten unter anderem einen gewissen Teil der Information des partiellen Einflusses der Entfernung auf die paarweise Anpassung. Aufgrund der zentralen Lage Nairobis in der Region sind die Entfernungen der entsprechenden Marktpaare eher kurz, doch der allgemeine Tansaniaeffekt beinhaltet eine Reihe von Marktpaaren, die mehr als $1000 \mathrm{~km}$ entfernt liegen (i.d.R. Paare zwischen Südtansania und Kenia). Der partielle Einfluß der Entfernung, der nichtlinear ist (Abbildung 4 und Abbildung 5) erscheint im parametrischen Modell nicht signifikant, da seine funktionale Form von den normalerweise in der Literatur zu Grenzeffekten verwendeten Funktionsformen nur inadäquat angenähert werden kann. Ihr wahrer Einfluß auf die paarweise Anpassung erscheint als leicht steigend von 47\% für sehr nahegelegene Marktpaare auf 48\% für etwa die durchschnittliche Entfernung aller Marktpaare $(650 \mathrm{~km})$ bis er ab etwa $1000 \mathrm{~km}$ um konstant einen Prozentpunkt je $100 \mathrm{~km}$ auf $42 \%$ bei $1500 \mathrm{~km}$ sinkt. 


\section{Schlußbetrachtung}

Dieser Aufsatz untersucht anhand von 85 Marktpaaren Preisdynamiken auf Maismärkten in Kenia, Tansania und Uganda, den drei größten Mitgliedsländern der Ostafrikanischen Gemeinschaft. Obwohl sie sich in vielerlei Hinsicht ähneln und auch räumlich nah zueinander liegen, unterscheiden sich doch ihre Politikstrategien und schwerpunkte hinsichtlich Agrarproduktion und -handel erheblich. Diese Arbeit zielt auf die Identifizierung von Faktoren, die das Ausmaß von Preisreaktionen auf Abweichungen vom Langzeitgleichgewicht (die Marktintegration) beeinflussen, und kommt zu politisch relevanten Ergebnissen.

Es werden ein parametrisches und ein semi-parametrisches partiell-lineares Modell mittels einer Kombination einer theorie- und statistikbasierten Modellauswahl identifiziert und geschätzt, wobei sich letzteres als das adäquatere herausstellt. Es werden ein signifikant negativer Effekt der Preisreaktion für Tansania, der einen signifikanten Grenzeffekt beinhaltet, und ein signifikant positiver Effekt für Nairobi in beiden Modellen identifiziert. Jedoch stellt sich heraus, daß die Entfernung einen nichtlinearen partiellen Einfluß ausübt. Dieser Einfluß weicht nachweislich signifikant von den üblichen parametrischen Modellierungen ab und kann daher nur durch das semi-parametrische Modell adäquat abgebildet werden. Der Tansaniaeffekt ist konsistent mit der Haltung der tansanischen Politik zum primären Sektor und zum Handel mit dessen Gütern sowohl im Inneren, Maßnahmen auf lokaler und regionaler Ebene, wie Besteuerung des Maishandels oder so genannte commodity boards widerspiegelnd, als auch an der Grenze zu Kenia, maßgeblich hervorgerufen v.a. durch die häufig angewandten Ausfuhrverbote. Dies belegt, daß die tansanischen Maßnahmen in dem Sinne, in dem sie angewendet wurden, erfolgreich waren. Es liegt jedoch jenseits des Rahmens dieser Studie, die Rolle dieser Politikmaßnahmen von der anderer transaktionskosten-erhöhender Faktoren zu isolieren; da dies jedoch vom politischen Standpunkt aus ein äußerst interessantes und relevantes Unterfangen wäre, gibt es hier noch reichlich Raum für weitere Forschung. In ähnlicher Weise erscheint der identifizierte Effekt für Nairobi sehr plausibel im Lichte der Größe und ökonomischen Bedeutung der Stadt im ostafrikanischen Raum. Der heterogene Grenzeffekt weist deutlich darauf hin, daß die Zollunion für einen Teil der Mitglieder, Kenia und Uganda, vollkommen umgesetzt wurde, während für Tansania offensichtlich noch erhebliche Anstrengungen zu unternehmen sind.

Die Ergebnisse der Untersuchung sind sehr überzeugend vor dem Hintergrund der landesspezifischen Politikansätze. Tansania weist im Inneren und an seinen Grenzen eine wesentlich schwächere Marktintegration als die restlichen Märkte auf, was darauf hinweist, daß in Teilen der Zollunion regional noch signifikant erhöhte Transaktionskosten existieren, da die tansanischen Politikmaßnahmen die gewünschten Ziele erreichten. Die Ergebnisse sind ökonomisch und politisch von Bedeutung, da sie interessante Einblicke in Ähnlichkeiten und Unterschiede in der Marktintegration von ostafrikanischen Maismärkten ermöglichen. 


\section{Literatur}

AwUoR, T. (2007): Review of Trade and Markets Relevant to Food Security in the Greater Horn of Africa. A special report by the Famine Early Warning Systems Network (FEWS NET) for USAID.

BARRETT, C.B. (2001): Measuring Integration and Efficiency in International Agricultural Markets. In: Review of Agricultural Economics 23(1): 19-32.

ENGEL, C. und J.H. Rogers (1996): How Wide Is the Border? In: American Economic Review 86(5): 1112-1125.

Evans, C. (2003): The Economic Significance of National Border Effects. In: American Economic Review 93(4): 1291-1312.

FACKLER, P.L. und B.K. Goodwin (2001): Spatial Price Analysis. In: B. Gardner and G. Rausser (Hrg.): Handbook of Agricultural Economics, Band 1, Elsevier, Amsterdam, The Netherlands: 971-1024.

HÄrdle, W., MÜller, M., Sperlich, S. und A. WerWATZ (2004): Nonparametric and Semiparametric Models. Springer Verlag, Heidelberg.

HELBLE, M. (2007): Border Effect Estimates for France and Germany Combining International Trade and Intranational Transport Flows. In: Review of World Economics 143(3): 433463.

HeLliwell, J.F. (1996): Do national boundaries matter for Quebec's trade? In: Canadian Journal of Economics 29: 507-522.

KInG, G., Honaker, J., Joseph, A. und K. Scheve (2001): Analyzing Incomplete Political Science Data: An Alternative Algorithm for Multiple Imputation. In: American Political Science Review 95(1): 49-69.

McCallum, J., 1995. National Borders Matter: Canada-U.S. Regional Trade Patterns. In: American Economic Review 85(3): 615-623.

Michigan STATE University (2008): Strengthening Food Security in Sub-Saharan Africa through Trade Liberalization and Regional Integration. Country Annexes. Maize Market Sheds in Eastern and Southern Africa. Bericht für die Weltbank, Washington.

OLPER, A. und V. RAIMONDI (2008): Explaining National Border Effects in the QUAD Food Trade. In: Journal of Agricultural Economics 59(3): 436-462.

Temu, A., Manyama, A. und A. Temu (2007): Alternative Staple Food Trade and Marketing Policy Interventions for Tanzania: A Country Level Assessment of the Maize Sub-Sector. Berichtsentwurf für die FAO, Daressalam, Tansania.

UniversitÄT VON TeXAs (2009): Perry-Castañeda Library Map Collection/ Africa Maps, http://www.lib.utexas.edu/maps/africa.html. Besucht im Januar 2009.

Vereinte NATIONeN (2009): Cartographic Section, Department of Field Support http://www.un.org/Depts/Cartographic/english/htmain.htm. Besucht im Januar 2009.

WeltBanK (2008): Non-Tariff Measures on Goods Trade in the East Africa Community. Weltbankbericht N. 45708-AFR, PRM2-AFCRI. Washington DC, USA. 


\section{Anhang}

Abbildung 6: Geschätzter nichtlinearer partieller Einfluß der Entfernung auf $S_{\alpha}^{A B}$ vs. die Daten

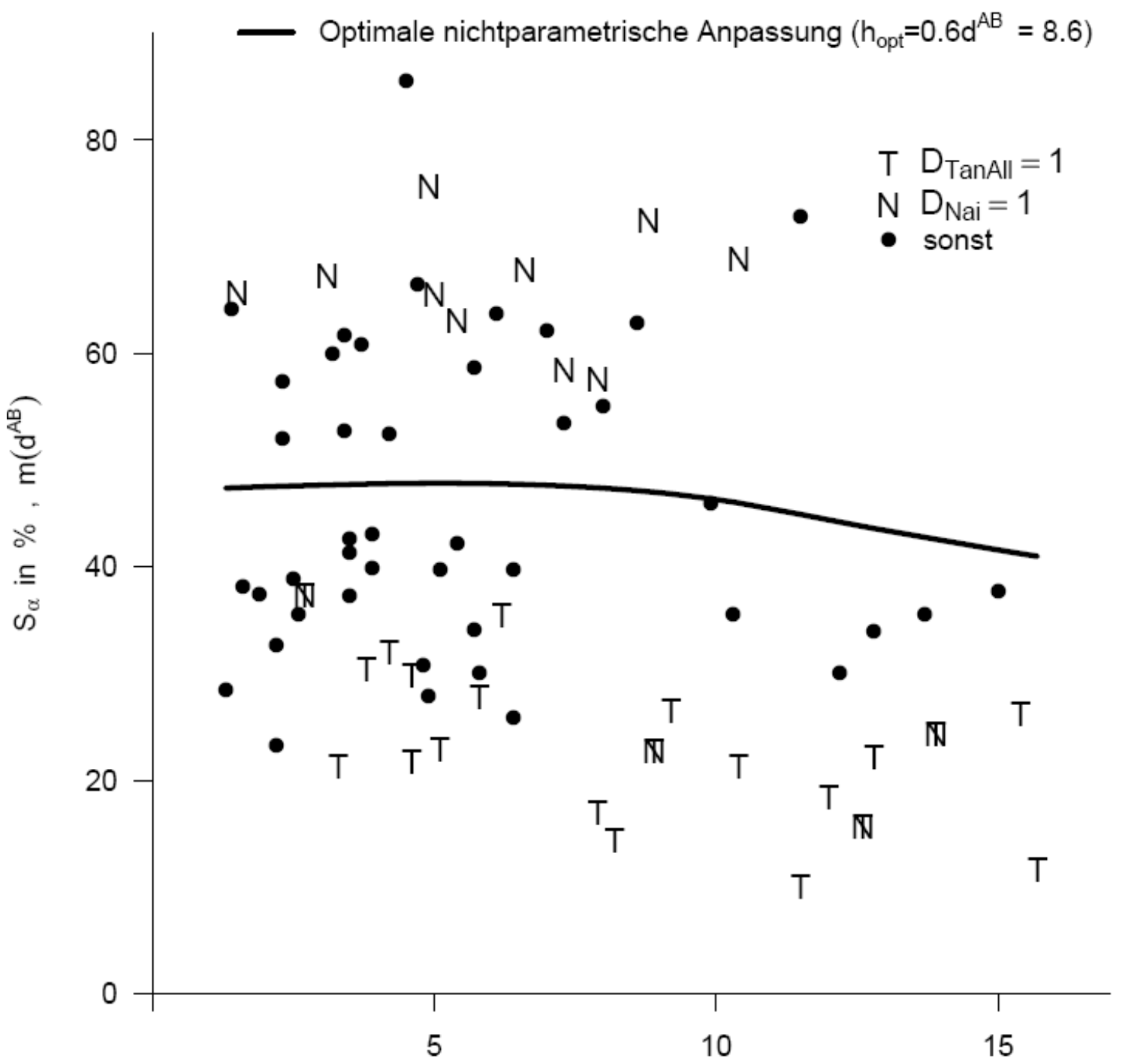

Entfernung zwischen den Märkten eines Paares in 100 km

Quelle: Eigene Darstellung. 


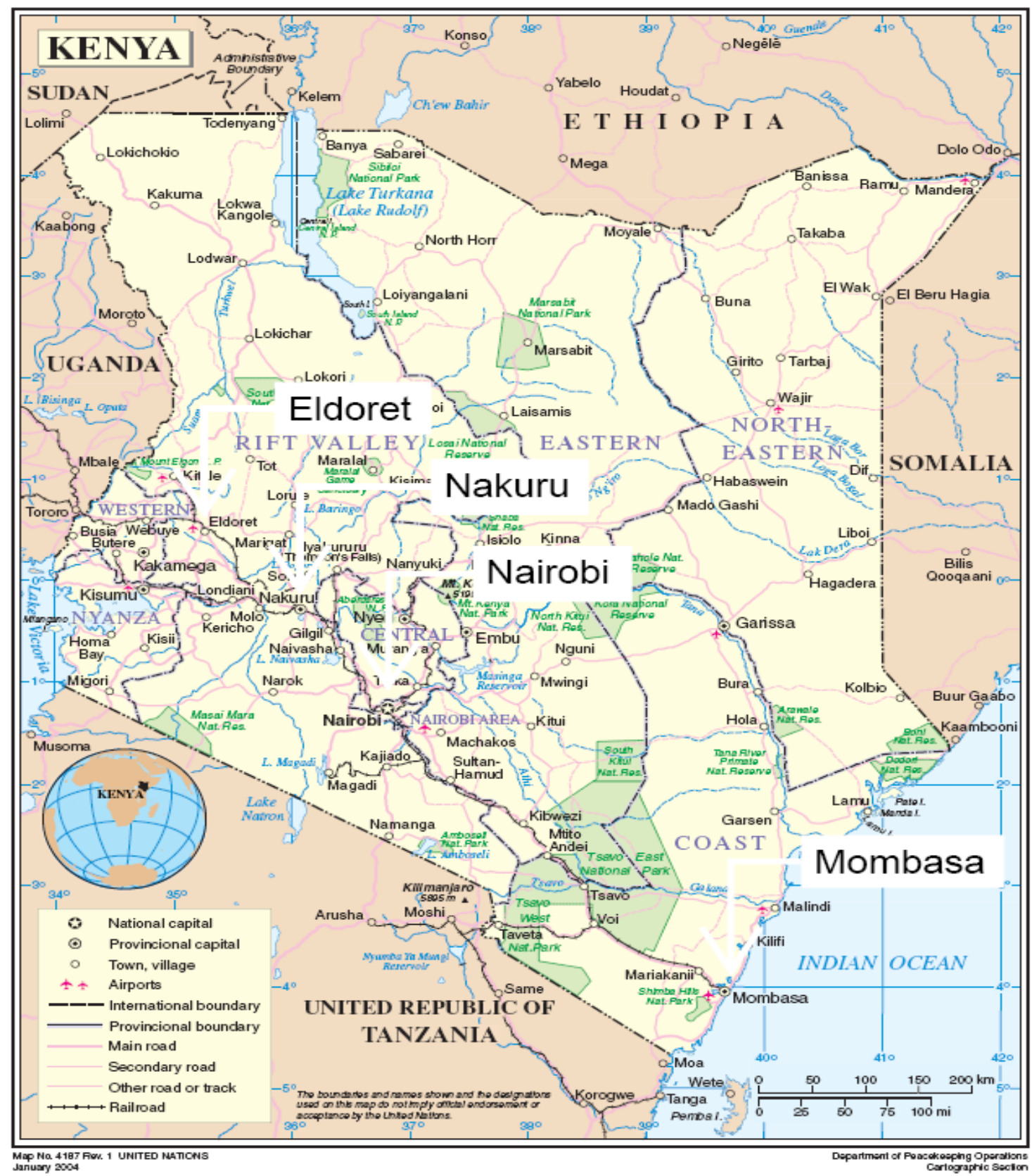

Quelle: Vereinte Nationen (2009) und eigene Darstellung. 


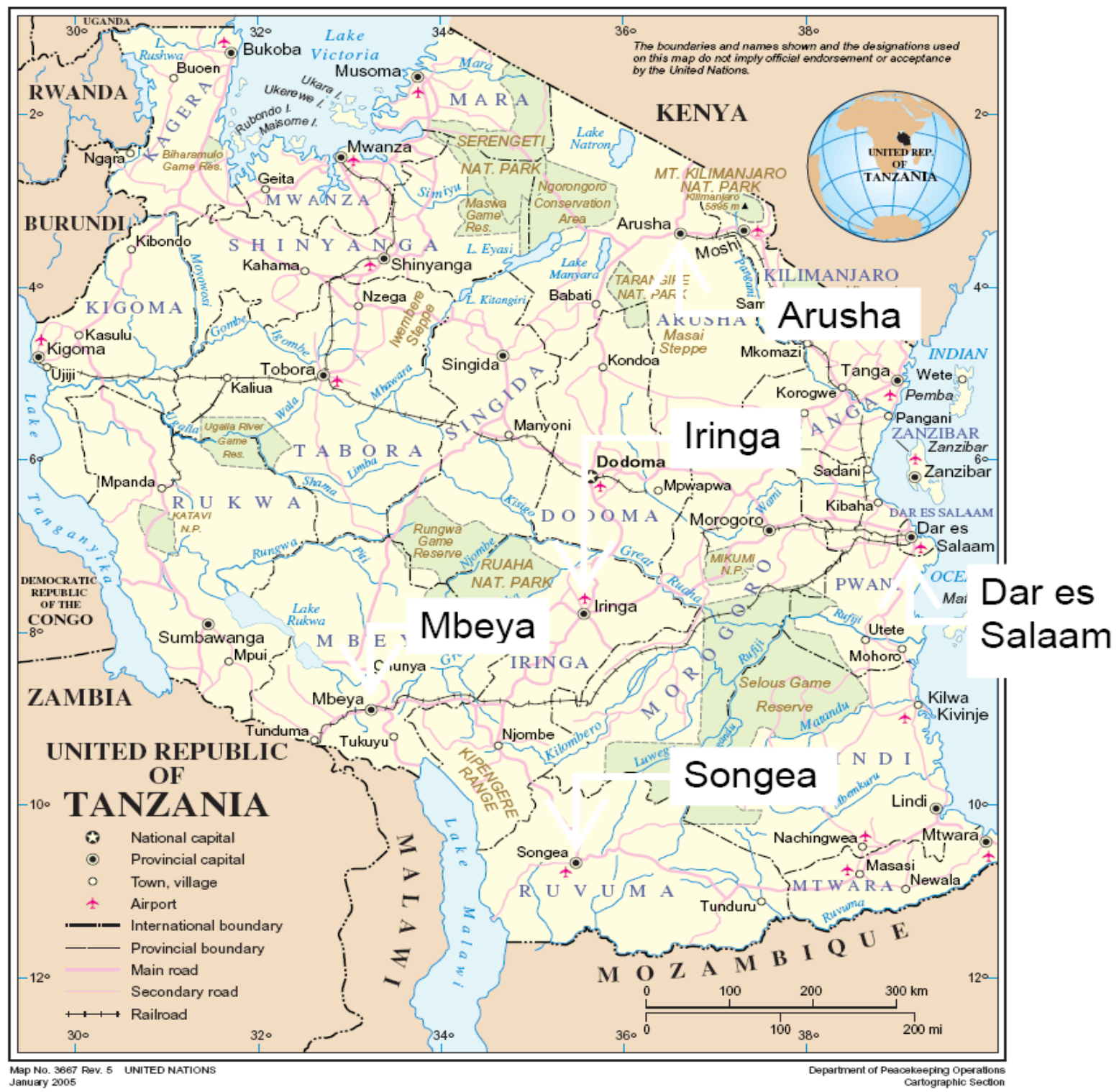

Quelle: Vereinte Nationen (2009) und eigene Darstellung. 


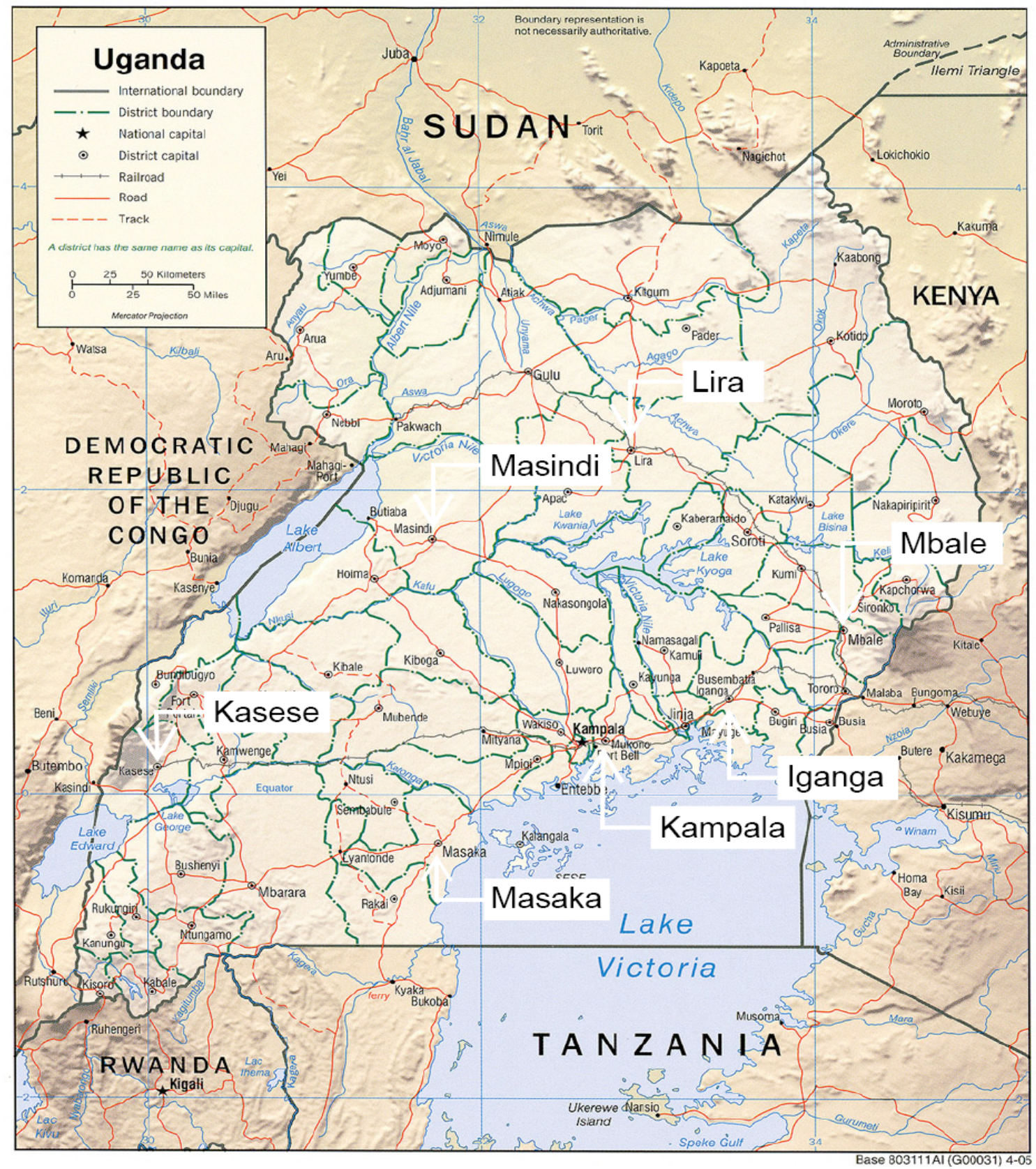

Quelle: Universität von Texas (2009) und eigene Darstellung. 


\title{
Appendix D
}

\author{
A Comparison of Threshold Cointegration and Markov- \\ Switching Vector Error-Correction Models in Price Trans- \\ MISSION ANALYSIS
}

This article is a cooperation with Stephan von Cramon-Taubadel. It has been published in the proceedings of the NCCC-134 Conference on Applied Commodity Price Analysis, Forecasting, and Market Risk Management. St. Louis, MO.

[http://www.farmdoc.illinois.edu/nccc134/paperarchive.html]

A short version of this research has been published under the title NonLineAR VECtor Error Correction Models in Price Transmission Analysis: ThreshOld Models vs. MARKov-Switching Models in the proceedings of the EAAE 2008 - XIIth Congress of the European Association of Agricultural Economists, Ghent, Belgium.

The article was presented at NCCC-134 Conference on Applied Commodity Price Analysis, Forecasting, and Market Risk Management St. Louis, Missouri, USA, April 21-22, 2008. The short version was presented as poster at EAAE 2008 - XIIth Congress of the European Association of Agricultural Economists, Ghent, Belgium, August 2629. 


\begin{abstract}
We compare two regime-dependent econometric models for price transmission analysis, namely the threshold vector error-correction model and Markov-switching vector error-correction model. We first provide a detailed characterization of each of the models, which is followed by a comprehensive comparison. We find that the assumptions regarding the nature of their regime-switching mechanisms are fundamentally different, so that each model is suitable for a certain type of nonlinear price transmission. Furthermore, we conduct a Monte Carlo experiment in order to study the performance of the estimation techniques of both models for simulated data. We find that both models are adequate for studying price transmission since their characteristics match the underlying economic theory and hence allow for an easy interpretation. Nevertheless, the results of the corresponding estimation techniques do not reproduce the true parameters and are not robust against nuisance parameters. The comparison is supplemented by a review of empirical studies of nonlinear price transmission, most of which apply the threshold vector error-correction model.
\end{abstract}

\title{
Keywords
}

comparison, market integration, Markov-switching vector error correction model, nonlinear time series analysis, price transmission, threshold vector error correction model

\section{Introduction}

Economists have devoted considerable attention to testing the Law of One Price (LOP) in a variety of settings, and agricultural economists in particular have generated an extensive literature on the empirical analysis of price transmission (PT) along spatial (prices for a homogeneous commodity at different locations - e.g. wheat in France and Germany) and vertical (prices for a commodity at different stages of processing - e.g. wheat-flour-bread) dimensions. Early studies focused on correlations or linear time series analysis involving prices, but in recent years attention has increasingly turned to the use of models that can capture the regime-dependent nature of relationships between prices. In a spatial context, the key insight, derived from Takayama and Judge (1971), is that prices will only co-move if spatial arbitrage conditions are binding (Baulch, 1994). If the difference between prices at two locations is greater than the cost of trade between these locations, then arbitrage will drive the price difference net of transaction costs to zero, and this equilibrating mechanism will lead to an observable relationship between the prices in question. If the difference between these prices is less than the transaction costs, however, arbitrage will not take place and in the simplest case the prices will move independently of one another. The result is a two-regime model of PT that extends to three regimes if the possibility of trade reversal is considered, and possibly more regimes if factors such as links to third markets or equilibrating mechanisms other than physical trade are accounted for. 
The threshold vector error-correction model has been used extensively in PT analysis (Goodwin and Piggott, 2001, etc.). Recently, Brümmer et al. (2009) proposed the use of the Markov-switching vector error-correction model to study price transmission in a vertical context between wheat and flour in Ukraine. So far no systematic attempt has been made to compare and contrast these models as regards their theoretical underpinnings and their performance and interpretation in the context of PT. In this paper we carry out such a comparison, in order to provide some indication regarding the common and differing features of both models. Both models allow for regimeswitching; does this characteristic imply that they may be used interchangeably and lead to congruent results? We show that this is not the case, and that each approach best suits particular analytical objectives in PT analysis. The comparison discusses the most important aspects for the empirical application of both models in PT analysis in detail in order to give some indication for the application of both models.

Section 2 outlines the relationship of both models to other time series models by introducing the class of nonlinear time series models in general and the subclass of threshold autoregressive models. These considerations are followed by a detailed characterization of the threshold and the Markov-switching vector error-correction model, respectively, by focusing on the basic idea, the model structure, the estimation and the interpretation of each. Section 3 provides a conceptual comparison of the characteristics of both models outlined before. It is supplemented by a simulation study which assesses the performance of the estimation methods of each model. The last section summarizes and draws conclusions. Appendix DI, pp. 197, provides a literature review of applications of the threshold vector error-correction model to PT analysis. Appendix DII, pp. 201, contains details on the simulation study.

\section{Model Review}

\subsection{Classification of nonlinear time series models}

Many model classes for nonlinear time series analysis were developed during the second half of the seventies and the eighties of the past century. ${ }^{2}$ Tong (1978) introduced the class of so-called threshold models. Fairly general formulations of nonlinear models have been developed by Priestley (1980b) (the class of state-dependent models) and Tjøstheim (1986) (the class of doubly stochastic models) which encompass a wide range of classes of less general models, including threshold models among others. Tong (1990) suggests a comprehensive classification of model classes for nonlinear time series analysis (Figure D.1). Model classes characterized by a specific functional relationship which do not contain other subclasses are called elementary model classes. On the next higher level, groups of such elementary classes, which are called first-generation mod-

$\overline{2}$ For a narrative about the "Birth of the Threshold Time Series Model" see Tong (2007). 
els, can be identified according to common properties. In turn, first generation models can be generalized in various ways. The resulting meta-classes such as the abovementioned state-dependent and doubly stochastic models are called second-generation models, which are very general in their specification and each of which includes various first-generation models. ${ }^{3}$ Among the first-generation models, a wide variety of model classes has been developed. Classes such as bilinear (BL) models, threshold autoregressive (TAR) models ${ }^{4}$ or autoregressive models with conditional heteroscedasticity $(\mathrm{ARCH})$ are examples. For the purpose of this paper, the class of TAR models is most interesting. It subdivides into the three groups of piecewise polynomial, piecewise linear and smooth autoregressive models depending on the functional relationship $f$ between the history $\left\{X_{p}\right\}_{p \in \mathbb{Z}, p<t}$ of the time series ${ }^{5}\left\{X_{t}\right\}_{t \in \mathbb{Z}}$ and its value $X_{t}$ at time $t$ :

$$
X_{t}=f(\underbrace{X_{t-1}, \ldots, X_{t-k}, \epsilon_{t-1}, \ldots, \epsilon_{t-k}}_{\text {history of the time series }})+\epsilon_{t}
$$

A general formulation of the threshold model might take the form

$$
\mathbf{X}_{\mathbf{t}}=\mathbf{A}^{\left(J_{t}\right)} \mathbf{X}_{\mathbf{t}-\mathbf{1}}+\mathbf{H}^{\left(J_{t}\right)} \boldsymbol{\epsilon}_{t}+\mathbf{C}^{\left(J_{t}\right)}
$$

where $\mathbf{X}_{\mathbf{t}}=\left(X_{t}, X_{t-1}, \ldots, X_{t-k+1}\right)^{\top}$ and $J_{t}$ denotes a random variable which takes one of the integer values $\{1,2, \ldots, l\}$ at each time $t . J_{t}$ is an indicator variable signaling the state (regime) in which the series $\left\{X_{t}\right\}$ is at time $t$. For a particular state $J_{t}=j$, the $(k \times k)$ non-random matrices $\mathbf{A}^{(j)}$ and $\mathbf{H}^{(j)}$ contain the autoregressive coefficients and the coefficients that reflect heteroscedasticity, respectively. The $(k \times 1)$ vector $\mathbf{C}^{(j)}$ comprises the constants of the relationship. $\left\{\boldsymbol{\epsilon}_{t}\right\}$ denotes a sequence of identically and independently distributed (iid) k-dimensional random vectors with zero mean and existing covariance matrix. Thus, for each state $J_{t}=j$ the relationship is locally linear ${ }^{6}$ with a particular set of coefficients and/or a constant.

The determination of $J_{t}$ remains unspecified in (D.2). One might think of various ways in which the states of $\left\{X_{t}\right\}$ are determined. This indicator variable is the key element of the nonlinear character of the equation; as Tong (1983) puts it, " $J_{t}$ indicates the mode of the dynamic mechanism". The realizations of $J_{t}$ at all time points $t$ form the series of the states (regimes) $\left\{J_{t}\right\}$ which is referred to as the regime-generating process (RGP)

3 However, Tong (1990), among others, questions their usefulness for practical analysis because of the high degree of generalization.

4 TAR models are called nonlinear mean reversion (NMR) models in real exchange rate analysis; compare, for example, Norman (2007) and O'Connel and Wei (2002). However, we will stick to the former term throughout this paper.

5 We use the abbreviated form $\left\{X_{t}\right\}$ for denoting a time series in this paper.

6 As Priestley (1980a) notes, the term local refers in this setting not to the proximity to a particular point in time but to a certain region of the state space of the series. Furthermore, linear refers to the constancy of parameters in such a region. Local linearity is thus the key property of TAR models, namely that their parameters are not constant over the whole range of observations, but take (constant) values depending on the current state/ the regime of the time series. Hence they are only constant within each state and called state-dependent or regime-dependent parameters. 


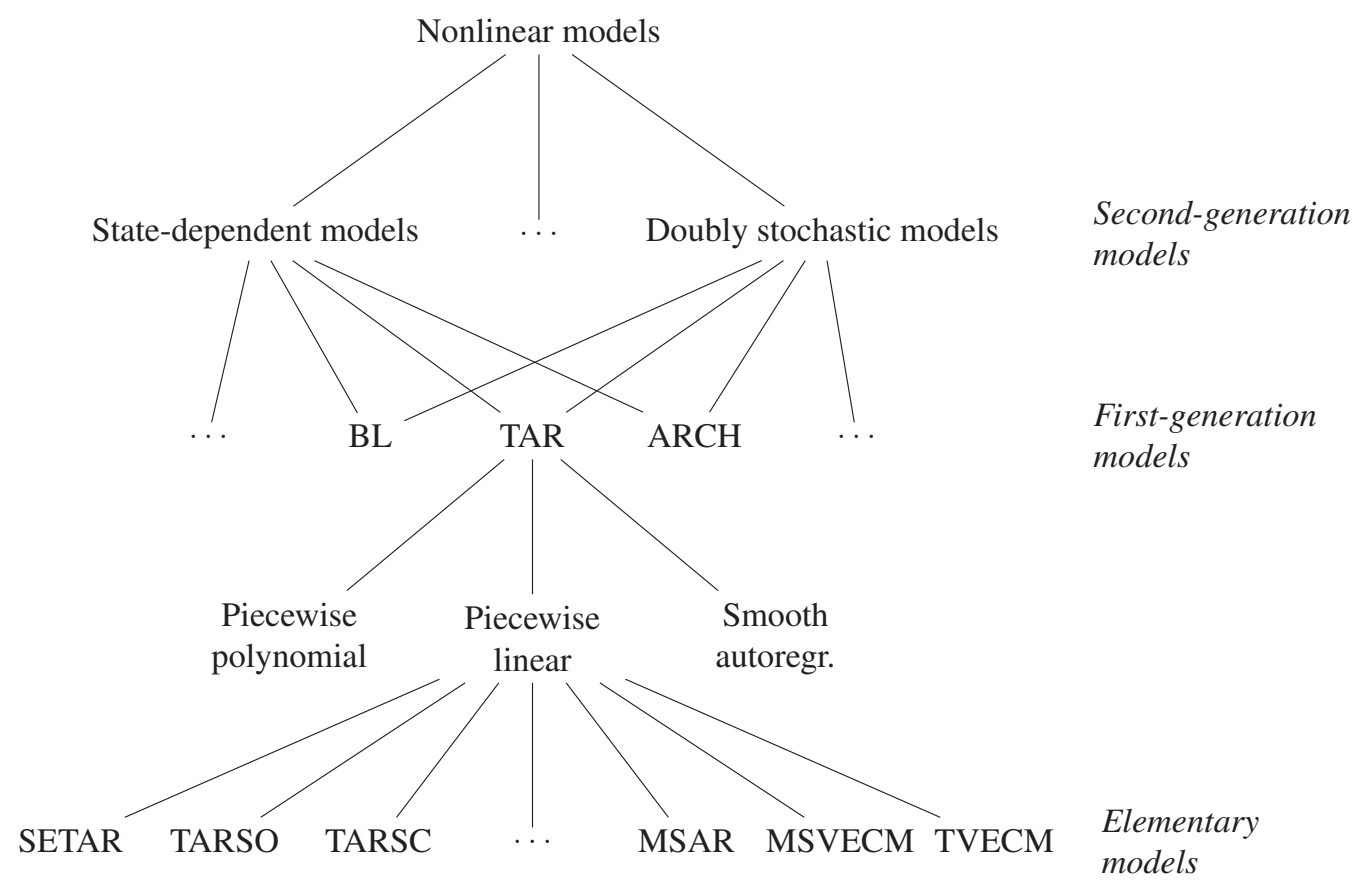

Figure D.1: Classification of Nonlinear Models after Tong (1990)

of the time series. This generation mechanism of the regime process characterizes elementary model classes within the class of piecewise linear TAR models. The state of a threshold model can be generated by one of the following basic mechanisms :

$$
\begin{aligned}
J_{t} & =f\left(X_{t-p}\right) \\
J_{t} & =f\left(Y_{t-q}\right) \\
J_{t} & =f\left(X_{t-p}, Y_{t-q}\right)
\end{aligned}
$$

where $t, p \in, q \in \mathbb{N}$ and $t>p, t>q$.

The first case refers to the endogenous determination of the regimes of $\left\{X_{t}\right\}$ by some part of its history. Tong (1990) calls this the class of self-exciting threshold autoregression models (SETAR) $)^{7}$ since the regimes of $\left\{X_{t}\right\}$ are completely generated by the series itself. The second case denotes the exogenous determination by some other series $\left\{Y_{t}\right\}$, lagged by $q$ periods, that is independent of $\left\{X_{t}\right\}$. One can think of a number of ways of exogenous determination. The most obvious generation mechanism is a second time series $\left\{Y_{t}\right\}$ which is known. Tong (1990) refers to this case as an openloop threshold autoregressive system (TARSO). If $\left\{Y_{t}\right\}$ itself follows a threshold model

\footnotetext{
7 The abbreviation may be complemented by the number of regimes and the lag length as $\operatorname{SETAR}\left(l ; k_{1}, k_{2}, \ldots, k_{l}\right)$ where $l$ denotes the number of regimes and $k_{j}, j=1,2, \ldots, l$ the lag-length in the $j^{\text {th }}$ state as, for example, in Tong (1990), or only by the number of regimes $\operatorname{SETAR}(l)$ as, for example, in Hansen (1999).
} 
and its regimes are exogenously determined by $\left\{X_{t}\right\}$, the model is called a closed-loop threshold autoregressive system (TARSC), i.e., each of the two series is determining the states of the other one. Another possibility, among others, is the determination of the states by a set of unknown (exogenous) variables which cannot be identified or measured for some reason so that only quantify conditional probabilities of staying in a state or switching to another can be quantified. Thus, the states of a series $\left\{X_{t}\right\}$ might be generated by a Markov chain. The resulting model is called Markov-switching autoregressive (MSAR) ${ }^{8}$ which can easily be transformed into the Markov-switching vector error-correction model (MSVECM). The third type of regime determination can be thought of as a mixture of the two above-mentioned ones in which the states of $\left\{X_{t}\right\}$ are determined by a combination of lagged values of the series itself and of some exogenous series $\left\{Y_{t}\right\}$. The case that the states of the second series $\left\{Y_{t}\right\}$ are in turn determined by a combination some lag of itself and of $\left\{X_{t}\right\}$ can be called simultaneous TARSC. If regressands of such a system are not expressed in levels but in differences, the resulting piecewise linear TAR model with mixed regime determination is called a threshold vector error-correction model (TVECM). Hence, the TVECM and the MSVECM both belong to the class of piecewise linear TAR models.

\subsection{Detailed Characterization}

\section{The Threshold Principle}

In a simple market setting it is often postulated that quantity demanded will equal zero above a certain price, or that quantity supplied will equal zero below a certain price. As a result, the functional relationship between quantity (supplied or demanded) and price will be subject to different regimes depending on whether the price is above or below certain values.

Such values are called thresholds. A threshold introduces nonlinearities into the functional relationship and "specifies the operation modes of the system" (Tong, 1990). The relationship between two or more variables might be locally linear ${ }^{9}$; however, globally it exhibits nonlinear behavior because of the existence of one or more structural changes in the relationship.

Tong (1990) notes that "threshold is a generic concept" resulting from the general property of saturation ${ }^{10}$, i.e., the structural changes as found, for example, in the mentioned quantity-price relationship. Tong defines the threshold principle as "the local approximation over the states, i.e., the introduction of regimes via thresholds."

\footnotetext{
8 A multidimensional version of this class can be seen in the Markov-switching vector autoregressive (MSVAR) models which are discussed in depth in Krolzig (1997).

9 Compare footnote 6 on page 165 for a definition of local linearity.

10 Tong (1990) and Tong and Lim (1980) provide a large number of examples in various disciplines of science.
} 
Such regime-dependent parameter stability of some time series is usually referred to as threshold behavior. Balke and Fomby (1997, and references therein) list several examples in which threshold behavior is found in economics, e.g. prices, inventories, consumer durables or employment.

\section{Threshold VECTOR ERROR-CORRECTION MODELS}

\section{Basic Idea}

Although Whittle (1954) was first to suggest a statistical model based on the threshold idea, the class of threshold models was formally introduced by Tong in 1978. He and many other researchers subsequently extended this area of research. Bhansali suggests that, as early as 1980, "commodity price series [are] a possible class of economic time series where applications of these models may be useful". In the second half of the 1980s, cointegration theory was developed to deal with the analysis of non-stationary time series. ${ }^{11}$ In 1997, Balke and Fomby published a paper on threshold cointegration in which they united both developments. Their essential insight is the assumption that the correction of deviations from the long-run equilibrium, i.e., the equilibrium errors, might display threshold behavior. The TVECM has attracted much attention in PT analysis, among other areas, since the publication of Balke and Fomby (1997). ${ }^{12}$

The possible existence of nonlinear PT was first hypothesized by Heckscher (1916). ${ }^{13}$ In the context of international trade, he proposed a band of inaction in which small deviations from the equilibrium price are not adjusted because transaction costs are higher than potential earnings due to the price differential. These transaction costs not only encompass transport costs, but also, for example, costs of searching, negotiating, insurance and risk premia. Heckscher termed the boundaries of this neutral band, in which prices are supposed to move freely, commodity points. In other words, the transmission of price signals between markets depends on whether deviations from the equilibrium price are inside the band of inaction or not, i.e., PT changes structurally depending on the magnitude of the deviations. Hence, PT is likely to follow threshold behavior. Such a regime-dependent nature of PT also results from the EnkeSamuelson-Takayama-Judge spatial equilibrium model formulated in Takayama and Judge (1971). This model implies that trade will only occur if the price spread of some homogeneous commodity between two spatially separated markets is at least as large as the transaction costs of trading between these two markets. Consequently, PT depends on the magnitude of the price spread, i.e., it shows regime-dependent

\footnotetext{
${ }^{11}$ Compare, for example, Engle and Granger (1987), Johansen (1995), Hendry and Juselius (2000) or Hendry and Juselius (2001).

${ }^{12}$ We provide a review of publications which study PT in commodity trade using mainly the TVECM in the econometric analysis in Appendix DI, pp. 197.

${ }^{13}$ This idea is based on the LOP as it was formulated by Marshall (1890, p. 325) who also mentioned the role of transaction costs.
} 
behavior.

Figure D.2 depicts the threshold behavior of PT. ${ }^{14}$ It shows the quantity traded $\operatorname{trade}_{t}^{A B}$ from market $A$ to market $B$ as a function of the price differential $p_{t}^{B}-p_{t}^{A}$ between $B$ and $A$. $\tau$ denotes the price differential above which trade takes place. Rational traders will only engage in trade if it is profitable, i.e., when they make a net profit. Thus, $\tau$ can be interpreted as the commodity point for trade from $A$ to $B$ which is equivalent to the transaction costs involved in the trading process. Price differentials below $\tau$ will not trigger trade flows and are not adjusted. However, if the price differential is greater than $\tau$, trade, by shifting supply from A to B, will cause $p_{t}^{A}$ to rise and $p_{t}^{B}$ to fall. This mechanism reduces the price differential in a process that will continue until it returns to $\tau \cdot p_{t}^{B}-p_{t}^{A}-\tau=0$ is therefore an equilibrium relationship. If both $p_{t}^{A}$ and $p_{t}^{B}$ are $I(1)$, it will be a cointegrating relationship, with an equilibrium error $p_{t}^{B}-p_{t}^{A}-\tau$ that is corrected by trade whenever it exceeds zero ${ }^{15}$; values of the error that are less than zero are not corrected. ${ }^{16}$ Hence, trade leads to the many times studied price adjustment process. Consequently, the magnitude of PT will differ depending on whether trade takes place or not, that is, PT shows regimedependent behavior. Thus, threshold models are both theoretically and intuitively appropriate in general for the analysis of PT. Moreover, the regressands are usually expressed in first differences, i.e., $\Delta p_{t}^{A}=p_{t}^{A}-p_{t-1}^{A}$ and $\Delta p_{t}^{B}=p_{t}^{B}-p_{t-1}^{B}$. The regimes of each price series, directly corresponding to the regimes of PT, are determined by the error-correction term, which is itself a function of both series. Thus, a simultaneous TARSC in the form of the TVECM is an appropriate model. Obstfeld and Taylor (1997) provide the first publication which explicitly refers to the hypothesis of Heckscher. O'Connel and Wei (1997) and Trenkler and Wolf (2003) derive this idea from economic theory. Several theoretical models in the area of real exchange rate analysis yield results in line with Heckscher's hypothesis; see, for example, Dumas (1992), Uppal (1993), Sercu et al. (1995), Coleman (1995, 2004).

\footnotetext{
${ }^{14}$ A cointegration vector $\boldsymbol{\beta}=(1-1)^{\top}$ is implicitly assumed here.

${ }^{15}$ Hence, the price spread $p_{t}^{B}-p_{t}^{A}$ is directly proportional to the equilibrium error. Since, for example, the price change in market $B \Delta p^{B}=p_{t}^{B}-p_{t-1}^{B}$ is a measure for trade from $A$ to $B$, the error-correction mechanism as depicted, for example, in Meyer (2004) corresponds to Figure D.2.

${ }^{16}$ However, negative values of the error are bounded from below by a second threshold which measures the transaction costs of trade in the opposite direction. This second threshold need not be of the same magnitude as the first, as, for example, the costs of moving up- as opposed to downriver or with and without backhauls might differ.
} 


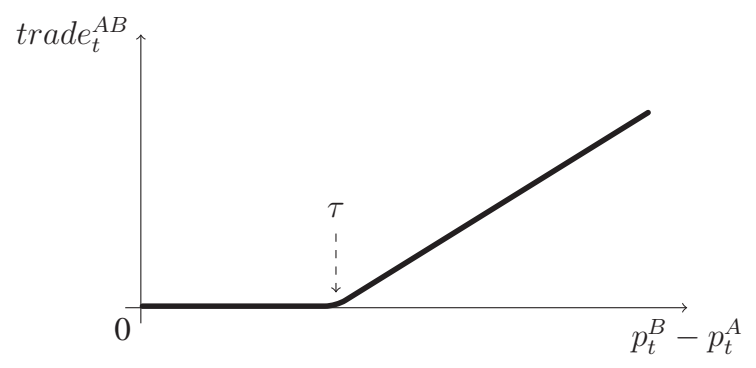

Figure D.2: Transactions Costs and Regime-Dependent PT

\section{Model Structure}

The TVECM may generally be formulated as follows ${ }^{17}$ :

$$
\begin{aligned}
\Delta \mathbf{p}_{t} & =\boldsymbol{\mu}^{(j)}+\boldsymbol{\alpha}^{(j)} \boldsymbol{\beta}^{\top} \mathbf{p}_{t-1}+\mathbf{C}^{(j)}(L) \Delta \mathbf{p}_{t}+\boldsymbol{\epsilon}_{t} \quad \text { if } \theta^{(j-1)}<\boldsymbol{\beta}^{\top} \mathbf{p}_{t-d} \leq \theta^{(j)} \\
& =\boldsymbol{\mu}^{(j)}+\boldsymbol{\alpha}^{(j)} e c t_{t-d}+\sum_{i=1}^{k-1} \boldsymbol{\Psi}_{i}^{(j)} \Delta \mathbf{p}_{t-i}+\boldsymbol{\epsilon}_{t} \quad \text { if } \theta^{(j-1)}<e c t_{t-d} \leq \theta^{(j)} \\
& =\boldsymbol{\mu}^{\left(J_{t}\right)}+\boldsymbol{\alpha}^{\left(J_{t}\right)} e c t_{t-d}+\sum_{i=1}^{k-1} \boldsymbol{\Psi}_{i}^{\left(J_{t}\right)} \Delta \mathbf{p}_{t-i}+\boldsymbol{\epsilon}_{t}
\end{aligned}
$$

where $\mathbf{p}_{t}=\left(\begin{array}{ll}p_{t}^{A} & p_{t}^{B}\end{array}\right)^{\top}$ is the vector of prices in markets $A$ and $B, t=1, \ldots, T$ denotes the time index and $j \in\{1,2, \ldots, l, l+1\}$ the index of the regimes. $\boldsymbol{\mu}^{(j)}$ denotes the regime-dependent mean where the superscript $(j)$ signals the regime-dependency of the parameter. ect $t_{t-d}=\boldsymbol{\beta}^{\top} \mathbf{p}_{t-d}$ denotes the deviation from the long-run equilibrium, i.e. the error-correction term lagged by $d$ periods. ${ }^{18} \boldsymbol{\beta}=\left(\begin{array}{ll}\beta^{A} & \beta^{B}\end{array}\right)^{\top}$ denotes the cointegration vector of the prices $\mathbf{p}_{t}$ and $\boldsymbol{\alpha}^{(j)}=\left(\boldsymbol{\alpha}^{A} \quad \boldsymbol{\alpha}^{B}\right)^{\top(j)}$ is called the loading vector. It contains the regime-dependent parameters characterizing to what extent the price changes $\Delta \mathbf{p}_{t}$ react on deviations from the long-run equilibrium lagged by $d$ periods. These parameters are interpreted as the magnitudes of error-correction of both prices which are equivalent to the speed (the rate) of price adjustment to the longrun equilibrium and characterize the regime-dependent magnitudes of $\mathrm{PT} \mathbf{C}^{(j)}(L)$ denote lag polynomials of order $k$ and, alternatively, the $\Psi_{i}^{(j)}$ are $(2 \times 2)$ matrices containing the autoregressive coefficients of each price difference (the coefficients for short-run adjustment of deviations). The errors $\boldsymbol{\epsilon}_{t}$ are $(2 \times 1)$ vectors of iid random variables with mean zero and finite covariance matrix $\Sigma$.

The values $\theta^{(j)} \in \mathbb{R}$ are ordered so that $\theta^{(0)}<\theta^{(1)}<\ldots<\theta^{(l)}<\theta^{(l+1)}$ where $\theta^{(0)}=-\infty, \theta^{(l+1)}=\infty$. They are called threshold parameters or, for short, thresh-

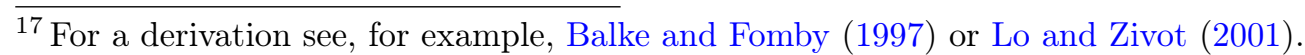

${ }^{18}$ Here it becomes obvious, that the threshold variable $e c t_{t-d}$ is a linear combination of the price series $\mathbf{p}_{t}$ and thus a function of those.
} 
olds. ${ }^{19}$ We impose the assumption on the thresholds to be time-invariant since this specification is almost exclusively used in applied research. ${ }^{20}$ The variable determining the relevant regime at time $t$ is called the threshold variable. ${ }^{21}$ It is assumed to be stationary and to follow a continuous distribution. $d \in$ is called the delay parameter. Alternatively, the model can be formulated using the indicator variable $J_{t}$ introduced in (D.2). It takes the value $j$ at time $t$ if $\theta^{(j-1)}<$ ect $t_{t-d} \leq \theta^{(j)}$.

Obviously, the nonlinear TVECM is a generalization of the linear vector error-correction model (VECM). Each threshold $\theta^{(j)}$ is only meaningful if

$$
0<\mathrm{P}\left(\theta^{(j-1)}<e^{e c t_{t-d}} \leq \theta^{(j)}\right)<1 .
$$

That is, only if realizations of the threshold variable occur with a probability larger than zero, i.e., are observable in each regime, the respective threshold exists. ${ }^{22}$ By introducing dummy variables for each regime, the model can be formulated more compactly in terms of a multivariate regression model similar to Hansen and Seo's (2002):

$$
\begin{aligned}
\Delta \mathbf{p}_{t} & =\boldsymbol{A}^{(1) \top} \mathbf{X}_{t-1} \mathbf{d}_{t}^{(1)}+\ldots+\boldsymbol{A}^{(l) \top} \mathbf{X}_{t-1} \mathbf{d}_{t}^{(l)}+\boldsymbol{\epsilon}_{t} \\
& =\sum_{j=1}^{l} \boldsymbol{A}^{(j) \top} \mathbf{X}_{t-1} \mathbf{d}_{t}^{(j)}+\boldsymbol{\epsilon}_{t} \\
& =\boldsymbol{A}^{\left(J_{t}\right) \top} \mathbf{X}_{t-1}+\boldsymbol{\epsilon}_{t}
\end{aligned}
$$

where $\mathbf{A}^{(j)}$ denotes a $((2 k+2) \times 2)$ matrix of coefficients. The vector of the regressors of (D.5) with $(2 k+2)$ elements is contained in $\mathbf{X}_{t-1}=\left(\begin{array}{llll}1 & \boldsymbol{\beta}^{\top} \mathbf{p}_{t-1} & \Delta \mathbf{p}_{t-1} \ldots \Delta \mathbf{p}_{t-k}\end{array}\right)^{\top}$. Furthermore, $\mathbf{d}_{t}^{(j)}=\mathbb{1}_{\left(\theta^{(j-1)}<e c t_{t-d} \leq \theta^{(j)}\right)}$ denotes the dummy variable signaling the $j$ 's regime of the series at time $t$ where $\mathbb{1}_{(\bullet)}$ is the indicator function. By expressing the regimes of the price series in terms of the indicator variable $J_{t}$, a special case of (D.2) is obtained.

\footnotetext{
${ }^{19}$ The thresholds $\theta^{(0)}$ and $\theta^{(l+1)}$ are usually not referred to as thresholds in the proper sense of the term. Rather, they represent some kinds of natural boundaries since the threshold variable of any meaningful model will take values between $-\infty$ and $\infty$. Hence, they also exist for each linear model and are only introduced for the sake of the generality of (D.4) - (D.6). In general, if we speak of thresholds we refer only to the inner ones, i.e., $\theta^{(1)}, \theta^{(2)}, \ldots, \theta^{(l)}$. Thus, in general, a TVECM of $s$ regimes has $s-1$ effective, i.e., inner thresholds and vice versa. Thus, $l$ denotes the number of effective thresholds.

${ }^{20}$ For models relaxing this restriction see, e.g., van Campenhout (2007), who models the threshold as a linear function of time, and Park et al. (2007), who derive formulae for dynamic thresholds varying on a daily basis.

${ }^{21}$ In the case of the traditional TVECM, the threshold variable is always the deviation from the long-run equilibrium $e c t_{t-d}$.

${ }^{22}$ If the realizations of the threshold variable are likely to occur only in one regime, no effective threshold exists and the TVECM in (D.5) simplifies to a linear VECM of the form $\Delta \mathbf{p}_{t}=\boldsymbol{\mu}+$ $\boldsymbol{\alpha} e c t_{t-d}+\sum_{i=1}^{k-1} \boldsymbol{\Psi}_{i} \Delta \mathbf{p}_{t-i}+\boldsymbol{\epsilon}_{t}$.
} 
In the analysis of PT, the thresholds are interpreted as the transaction costs for moving a homogeneous commodity between any pair of markets which introduce the nonlinear behavior into the PT process. The error-correction mechanism is usually assumed to react immediately one period after some deviation from equilibrium, i.e., the delay parameter is usually assumed to equal one and the error-correction term becomes $e c t_{t-1}$. Furthermore, the number of regimes is restricted, often set to either two or three, implying one or two thresholds respectively. ${ }^{23}$ In line with the above-mentioned theoretical background, a TVECM(3) has much appeal since it accounts for trade in both directions between two spatially separated markets. ${ }^{24}$

Balke and Fomby (1997), as well as Lo and Zivot (2001), suggest certain restrictions on the model which might be particularly suitable for applied analysis. The two prices can, based on Heckscher's supposition, be expected not to be cointegrated inside the "band of inaction" spanned by the two transaction costs, implying that the price differences $\Delta \mathbf{p}_{t}$ move as random walks around zero. Consequently, no error-correction takes place in regime $j=2$ between the two thresholds, i.e., $\boldsymbol{\alpha}^{(2)}=\mathbf{0}$, and the regimedependent mean equals zero $\boldsymbol{\mu}^{(2)}=\mathbf{0}$. Depending on the center of attraction of the error-correction mechanism, special cases of the model can be distinguished. If the errors are corrected toward a band around the long-run equilibrium which is spanned by the regime-specific means $\boldsymbol{\mu}^{(1)}$ and $\boldsymbol{\mu}^{(3)}$, the model is called a BAND-TVECM as formulated in (D.11). ${ }^{25}$ However, if the errors are corrected toward the long-run equilibrium itself, implying $\boldsymbol{\mu}^{(1)}=\boldsymbol{\mu}^{(3)}=0$, the model is called an EquilibriumTVECM (EQ-TVECM). Moreover, the model is called continuous if $\boldsymbol{\mu}^{(1)}=-\boldsymbol{\alpha}^{(1)} \theta^{(1)}$ and $\boldsymbol{\mu}^{(3)}=-\boldsymbol{\alpha}^{(3)} \theta^{(2)}$. If both (effective) thresholds are of the same magnitude, i.e., if $-\theta^{(1)}=\theta^{(2)}$, implying identical transaction costs in both directions of trade, the model is called symmetric.

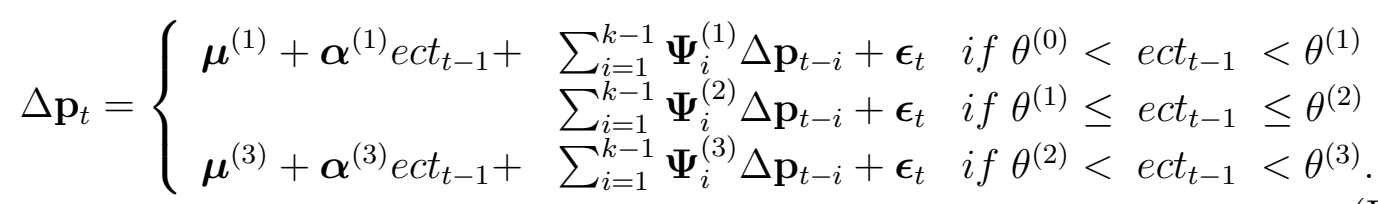

Figure D.3 depicts a realization of an EQ-TVECM characterized by three regimes. The regime $J_{t}$ depends exclusively on the magnitude of the first lag of the errorcorrection term $e c t_{t-d}$. The price series $p_{t}^{A}$ and $p_{t}^{B}$ are plotted in the bottom panel. The variable causing regime switches is deviation from the long-run equilibrium, i.e.,

\footnotetext{
${ }^{23}$ In order to refer to the number of regimes, the name of the specified model is sometimes supplemented by this number, for example a TVECM with $l$ thresholds has $l+1$ regimes and can be denoted by TVECM $(l+1)$ or $\operatorname{TVECM}_{l+1}$.

${ }^{24} \operatorname{A} \operatorname{TVECM}(2)$ where $\theta^{(1)}=0$ is suitable for the study of asymmetric PT, see, e.g., Chen et al. (2005).

${ }^{25}$ Note that the inequality signs differ slightly here in comparison to the above models since in price transmission analysis the edge of the band is considered to belong to the band since in this case the price differential equals the transaction costs. Nonzero profits to be exploited for traders only emerge in the case that the price differential is larger than the transaction costs.
} 
the error-correction term ect $t_{t}$ which equals the difference between the prices at each time $t$. It is separately plotted in the middle panel. If it is either smaller or larger than the lower $\theta^{(1)}$ or the upper threshold $\theta^{(2)}, J_{t}$ takes the values $j=1$ or $j=3$, respectively, and error-correction toward zero takes place. However, prices move independently inside the band spanned by the two thresholds since $\boldsymbol{\alpha}^{(2)}=0$. Whenever the threshold variable $e t_{t}$ crosses one of the thresholds, the regime switches after a lag of $d$ periods to the new regime as depicted in the middle and the upper panel of Figure D.3. ${ }^{26}$ The parameters of most interest in applied analysis are the thresholds $\theta^{(j)}$, the loading vectors $\boldsymbol{\alpha}^{(j)}$ and the cointegration vector $\boldsymbol{\beta}$.

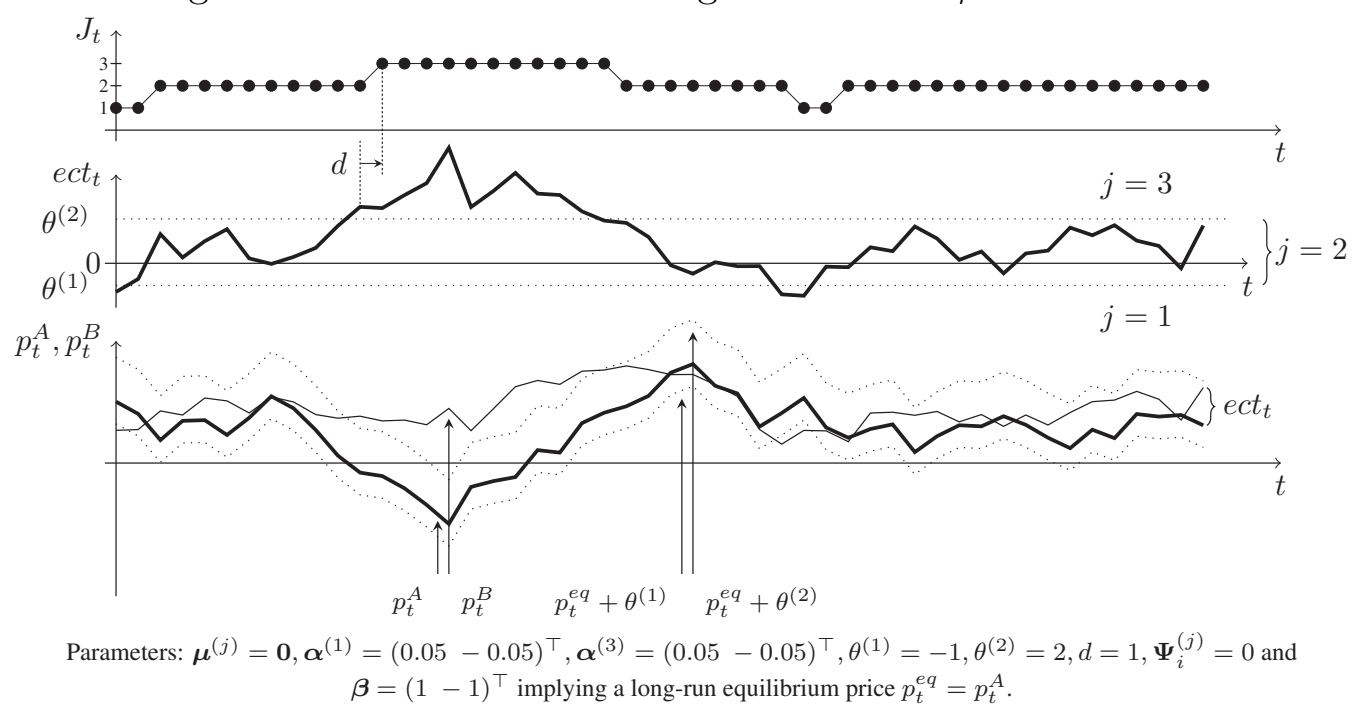

Figure D.3: Realization of a $\operatorname{TVECM}(3)$

\section{Estimation}

Several authors have developed estimation techniques for threshold models, either in general or for the TVECM in particular. Tong (1978) recommends the Entropy Maximization Principle based on the Akaike Information Criterion (AIC) for the estimation of a general TAR model. Tsay (1989), Chan (1993) and Hansen (2000) propose approaches for threshold models with two regimes. Tsay (1998) shows that, asymptotically, the estimates of this sequential conditional multivariate least squares estimation are strongly consistent and that the estimated coefficients $\mathbf{A}^{(j)}$ in equation (D.9) are independent of the thresholds $\theta^{(j)}$ and the delay parameter $d$ and normally distributed.

Balke and Fomby (1997) suggest conditional least squares estimation for TAR models applicable to any number of thresholds and delay parameters. Obstfeld and Taylor (1997, Appendix A) give a detailed description of their applied maximum likelihood

\footnotetext{
${ }^{26}$ The rationale for such a lag is that markets need some time to react. Nevertheless, this time may depend on the product traded, the market infrastructure and the socio-economic environment of the market.
} 
estimation technique. Hansen (1999) presents an estimation technique for SETAR models with two or more regimes based on sequential conditional least squares estimation through concentration. Lo and Zivot (2001, Appendix A) suggest a combination of the methods of Hansen (1999) and Tsay (1998) for estimating one threshold and the delay parameter of a multivariate TVECM. Hansen and Seo (2002) propose a maximum likelihood estimation procedure for the TVECM for the bivariate case, i.e., with two regimes, which allows for the simultaneous estimation of the cointegration vector and the threshold, and provide a detailed description of the algorithm proposed.

Table D.1 summarizes estimation approaches of selected publications in chronological order. It displays information on the underlying model such as the model class, the number of estimated thresholds $l$, and potential restrictions on the delay parameter $d$. Moreover, it mentions whether the estimation follows the maximum likelihood or the least squares principle. The latter is referred to differently in the literature as sequential, iterative or conditional (multivariate) least squares. ${ }^{27}$ This is complemented by information on the optimization method such as the considered optimization criterion, i.e., the objective function, its parameters, and the type of the optimization. The RSS criterion, in contrast to the log-determinant of the variance-covariance matrix, ignores correlations across the regimes' equations. Nevertheless, Serra and Goodwin (2002) have shown that both criteria yield the same estimation results and might thus be considered to be equivalently suitable.

\begin{tabular}{l|lcccccc}
\hline Publication & Model & $l$ & $d$ & Principle & Crit. & Par's & Optim. \\
\hline Balke and Fomby (1997) & SETAR & any & est. & LS & RSS & $\theta, d$ & Min \\
Obstfeld and Taylor (1997) & SETAR & $1^{28}$ & 1 & ML & LR $^{29}$ & $\theta$ & Max \\
Hansen (1999) & SETAR & $0-2$ & est. & LS & RSS & $\theta, d$ & Min \\
Lo and Zivot (2001) & TVECM & 1 & est. & LS & RSS & $\theta, d$ & Min \\
Hansen and Seo (2002) & TVECM & 1 & 1 & ML & $\log |\Sigma|$ & $\theta, \beta$ & Min \\
\hline
\end{tabular}

Note: $l$ denotes the number of thresholds (compare footnote 19, page 171), $d$ the delay parameter, $M L$ maximum

likelihood, $L S$ least squares, $R S S=\operatorname{trace}(\Sigma)$ residual sum of squares, $\log |\Sigma|$ the $\log$ determinant of the

variance-covariance matrix of the residuals; $\theta$ means threshold, est. estimated and $\beta$ the cointegration vector.

\section{Table D.1: Estimation Approaches of Selected Publications}

The functions of the presented criteria will usually not be smooth. ${ }^{30}$ Hence, a grid search algorithm in the form of SCLS is suitable for optimization. Its dimension depends on the number of parameters of the optimization criterion. ${ }^{31}$ The challenge

\footnotetext{
${ }^{27}$ We refer to the method as sequential conditional least squares (SCLS) throughout this paper.

${ }^{28}$ Obstfeld and Taylor estimate a symmetric TVECM(3), meaning that in absolute terms, only one symmetric threshold is to be estimated.

${ }^{29} L R$ denotes the likelihood ratio between a $\operatorname{SETAR}(3,1,1,1)$ and an $\operatorname{AR}(1)$. The latter model might also be called a SETAR $(1,1)$; for the notation of SETAR models, see footnote 7, p. 166.

${ }^{30}$ For examples of the shape of such criterion functions, see Hansen and Seo (2002, p. 298).

31 The higher the dimension, the higher the computational costs. Several authors have suggested alternatives; see, for example, Hansen and Seo (2002), Lo and Zivot (2001), Hansen (1999), Bai and Perron (1998), Bai (1997), or Dorsey and Mayer (1995).
} 
of estimation in this context, consists in the fact that the unknown parameters of the model depend on each other. The coefficients matrices $\mathbf{A}^{(j)}$ in (D.9), in addition to the variance-covariance matrix $\boldsymbol{\Sigma}$, depend among other things on the unknown thresholds $\theta^{(j)}$; however, the former are a precondition for estimating the latter.

The basic idea of the grid search is very pragmatic. In order to break the "vicious circle", the parameters of the optimization criterion, i.e., the thresholds among others, are pretended to be known and are set to some constants. Conditional to the combination of the chosen optimization parameters, the remaining model parameters (i.e., the coefficients matrices $\mathbf{A}^{(j)}$ and the variance-covariance matrix $\boldsymbol{\Sigma}$ ) and the optimization criterion are computed. The computation is repeated for a number of combinations of possible values of the optimization parameters, and the criterion is evaluated. ${ }^{32}$

Candidate values of the optimization parameters are generated by an evenly spaced grid across the empirical support of the threshold variable and potentially a reasonable range of the criterion's other parameters. The combination which optimizes the criterion represents the final estimates of the optimization parameters. Conditionally on these, the final estimates of the remaining model parameters are obtained. In case of the maximum likelihood approach of Hansen and Seo (2002), this idea is called concentrated or profile likelihood.

For practical computation, the constraint formulated in (D.7) has to be accounted for in order to ensure a reasonable number of observations for the estimation of $\mathbf{A}^{(j)}$. It is modified in the following way to ensure a minimal proportion of observations in each regime,

$$
\pi_{0}<\frac{T_{j}}{T}<1-l \cdot \pi_{0}
$$

where $T_{j}$ denotes the number of observations in regime $j$, and $l$ the number of thresholds of the model. The trimming parameter $\pi_{0}$ is usually set to 0.05 or 0.1 .

\section{Interpretation}

As sketched above, the TVECM specification, as long as the model is assumed or tested to have two effective thresholds, has an immediate economic motivation, namely the concept of commodity points suggested by Heckscher which corresponds to the transaction costs in a Enke-Samuelson-Takayama-Judge spatial equilibrium. Price adjustment as a consequence of trade only takes place if the price spread between two markets exceeds the "band of inaction". The latter is delimited by the two estimated thresholds. Prices move independently within this band and are not cointegrated.

The thresholds are interpreted as transaction costs which render trade costly and thus

$\overline{32}$ Detailed accounts of the algorithm are given, for example, in Serra and Goodwin (2003) and Park et al. (2007). 
inhibit it to a certain extent. Hence, the assumption that the thresholds are symmetric around the equilibrium error may not be appropriate in every case, since economic theory does not give indications for this assumption, and transaction costs are likely to be direction-specific (e.g. backhaul). The transaction costs encompass many more aspects than the expenses for transportation. They can be any costs with respect to temporal and financial expenses connected with the search for information, financing the trading process and legal duties (Shepherd, 1997). Barrett (2001) provides an extensive discussion of the components of such costs. A large part consists, of course, of freight rates. Additionally, variable costs associated with insurance, financing or contracting are relevant. Exogenous costs such as underwriting fees or testing charges might apply. Furthermore, average duties on the product and immeasurable transaction costs such as opportunity and search costs or risk premia might also play a role.

Consequently, if the deviation ect $t_{t}$ from the long-run equilibrium, as depicted in the middle panel of Figure D.3, is less than the transaction costs $\theta^{(1)}$ of trade from market $B$ to market $A$, it is corrected upward toward zero, i.e., the long-run equilibrium, by trade into this direction. If it exceeds the transaction costs $\theta^{(2)}$ of trade from $A$ to $B$, then it is corrected downward toward zero. Within the band spanned by the two transaction costs there is no incentive for trade in either direction. Prices move independently in this corridor, hence $\boldsymbol{\alpha}^{(2)}=\mathbf{0}$, until the deviation from their long-run equilibrium exceeds either of the transaction costs once more. At this point trade becomes profitable and the deviation decreases in the following periods. In other words, error-correction takes place.

To our knowledge, only very few papers opt for a broader interpretation of the estimated thresholds that encompasses more than transaction costs. Trenkler and Wolf (2003) suggest an extension of the interpretation of these parameters (which they call cost parameter) to social, cultural and technical aspects. Moreover, they discuss the potential impact of nominal fixed transaction costs on market integration when aggregate price levels fluctuate. Obstfeld and Taylor (1997) note that the estimated thresholds, which they call commodity points in line with Heckscher's terminology, may reflect more aspects than costs of transport and restrictions to trade. They refer to this additional component as sunk costs of arbitrage. O'Connel and Wei (2002) provide a comprehensive discussion of the importance of fixed and variable market frictions for deviations from the LOP. They hypothesize that costs connected to the change of preferences and technology, such as costs of labor migration or of entering and exiting a market, may also be relevant. Coleman $(1995,2004)$ addresses this argument as well. 


\section{Markov-Switching Vector Error-Correction Models}

\section{Basic Idea}

The underlying concept of the MSVECM emerged in the area of the econometrics of time-varying parameters. Goldfeld and Quandt (1973) developed a switching regression model characterized by parameter changes that are governed by a Markov chain. Hamilton (1989) extended this approach to the analysis of time series. A nonlinear VECM whose equilibrium errors follow a Markov process is suggested by several authors. Furthermore, the model is extended to the cointegration framework. Jackman (1995) proposes such a model for analyzing the determinants of presidential approval in the United States. Krolzig $(1996,1997)$ develops the MSVECM as a special case of the more general Markov-switching vector autoregression model, whereas Hall et al. (1997) apply a MSVECM to the analysis of house prices in the United Kingdom. ${ }^{33}$ Applications of the model are mainly found in business cycle and financial research, e.g. Krolzig and Toro (2001), Francis and Owyang (2003), or Psaradakis et al. (2004a), the latter suggesting further applications. Krolzig et al. (2002) analyze the British labor market. In PT analysis, the model is much less frequently applied than the TVECM; Brümmer et al. (2009) propose it to analyze vertical price transmission between wheat and flour in Ukraine.

As mentioned above, the MSVECM, as an elementary subclass of TAR models, is in general suitable for the analysis of PT due to the threshold behavior of trade as depicted in Figure D.2. Hamilton (1989) characterizes the particular form of nonlinearities that Markov-switching models are suitable for as "discrete shifts in regime-episodes across which the dynamic behavior of the series is markedly different". Hamilton (1994, ch. 22.4) emphasizes the inherent appealing characteristics of comprehensiveness and great flexibility of this model class. Hamilton and Raj (2002b) mention that "normal behavior of economies is occasionally disrupted by dramatic events that seem to produce quite different dynamics for the variables that economists study". Moreover, Psaradakis et al. (2004a) note that the MSVECM is "best suited to situations where the change in regime is triggered by a sudden shock to the economy, situations which might not be adequately described by models with smooth transitions or threshold effects".

The MSVECM can be characterized as a TAR model with exogenous determination of the states, that is, the regimes are not a function of the analyzed price series themselves but of external determinants which, in contrast, do not have to be observed. Such determinants might act as general driving forces of trade, prices and a number of

\footnotetext{
${ }^{33}$ They suggest two specifications of the model with, first, constant transition probabilities and, second, transition probabilities as functions of the equilibrium error. However, we focus in this paper only on the former case which represents the simplest form of the MSVECM with time-invariant transition probabilities. For extensions of this framework, see also, for example, Diebold et al. (1994), Hamilton and Raj (2002a) or Camacho (2005).
} 
further economic variables. In the context of PT, regime-switching seems plausible, which may not exclusively be determined by the equilibrium error, but rather by the "general state" of the trading process or even of the surrounding political or economic system. Price transmission behavior is likely to change temporarily due to external factors such as general characteristics of the political economic system. Raj (2002) mentions national policy changes, economic recessions, financial panics or wars in the context of business cycles. Further "sources of abrupt change" such as government actions in the form of the introduction or elimination of legal regulations are alluded to in Hamilton (1995). Chamley (1999) shows that a unique equilibrium may exist in a world characterized by imperfect information showing episodes of high and low economic activity which may, among others, result in differing equilibrium adjustment and short-run dynamics of PT. Furthermore, he shows that switches between such regimes occur randomly.

For example, one could think of traders' temporary insecurity about the future due to elections or turmoil in politically unstable countries, or due to exceptional positive or negative expectations about the near economic future, such as forecasts of strong price rises. ${ }^{34}$ Hence, traders face, for such limited periods, quite differing conditions ranging from increased uncertainty to the impossibility of trade and are likely to show, as a consequence, temporarily differing behavior. Such periods are hardly measurable. Agricultural scandals as they occasionally occur in Europe and the Unites States, resulting in at least temporary changes of consumer demand, may lead to transitionally different transmission of price signals. Further events such as temporal or new legal regulations, crop failures, or transient demand changes are likely to change the "normal" trade dynamics in an abrupt manner. ${ }^{35}$ Trade and hence price adjustment dynamics are furthermore subject to the asymmetries of the business cycle which were first hypothesized by Keynes (1936).

The importance of a further factor is stressed in the business cycle literature. Economic behavior and thus regime switches might by driven by extrinsic uncertainty in the sense of "random phenomena that do not affect tastes, endowments, or production possibilities" (Cass and Shell, 1983). This uncertainty is referred to in the literature as nonfundamentals, market psychology, animal spirits, sunspots or self-fulfilling prophecies. Several publications show that these phenomena are apt to create business cycle fluctuations in the absence of shocks to fundamentals of the economy, see, for example, Azariadis (1981), Cass and Shell (1983), Howitt and McAfee (1992), Jeanne and Masson (2000) or Thomas (2004). Hamilton and Raj (2002b) see the cause of such potential impact in the fact that "agents' believe such nonfundamentals affect aggregate economic activity". Such phenomena are likely to cause regimes in trade processes and also in PT, as well as random switches between them. ${ }^{34}$

\footnotetext{
${ }^{34}$ For an example, see Agra Europe (2008, middle of page M/3): "Importers still panic buying...".

${ }^{35}$ Martinez Peria (2002) aims at identifying speculative attacks on the European Monetary System during the first half of the 1990s by modeling a tranquil versus a speculative regime.
} 
In general, the MSVECM is formulated identically to the TVECM in (D.6):

$$
\Delta \mathbf{p}_{t}=\boldsymbol{\mu}^{\left(J_{t}\right)}+\boldsymbol{\alpha}^{\left(J_{t}\right)} \boldsymbol{\beta}^{\top} \mathbf{p}_{t-1}+\sum_{i=1}^{k-1} \boldsymbol{\Psi}_{i}^{\left(J_{t}\right)} \Delta \mathbf{p}_{t-i}+\boldsymbol{\epsilon}_{t}
$$

The number of regimes is denoted with $M$ so that $J_{t}=j \in\{1,2, \ldots, M\}$. The model can, of course, be written compactly, as in (D.10). Each regime-dependent variable takes a certain value depending on the value of the indicator variable $J_{t}$ at time $t$, for example $\boldsymbol{\alpha}^{\left(J_{t}\right)}=\boldsymbol{\alpha}^{(j)}$ if $J_{t}=j$, i.e.,

$$
\boldsymbol{\alpha}^{\left(J_{t}\right)}= \begin{cases}\boldsymbol{\alpha}^{(1)} & \text { if } J_{t}=1 \\ \vdots & \\ \boldsymbol{\alpha}^{(M)} & \text { if } J_{t}=M\end{cases}
$$

The regimes $j$ of the MSVECM (D.13) are thought of as determined by a probabilistic process which has $M$ states; they are assumed to be realizations of a latent $M$-state Markov chain with discrete state space in discrete time. The regime-dependent parameters are constant in each state but are allowed to change across states. Hence, each state of the underlying Markov chain directly corresponds to a regime of PT. Furthermore, the chain determines the regime switching.

The key element of the model is the $(M \times M)$ transition matrix $\Gamma$ which contains the transition probabilities $\gamma_{h j}$ for switching from state $h$ to state $j$

$$
\boldsymbol{\Gamma}=\left(\begin{array}{cccc}
\gamma_{11} & \gamma_{12} & \cdots & \gamma_{1 M} \\
\gamma_{21} & \gamma_{22} & \cdots & \gamma_{2 M} \\
\vdots & \vdots & \ddots & \vdots \\
\gamma_{M 1} & \gamma_{M 2} & \cdots & \gamma_{M M}
\end{array}\right)
$$

where $\gamma_{h j}=\operatorname{Pr}\left(J_{t+1}=j \mid J_{t}=h\right)$. The Markov chain is assumed to be homogeneous, that is, the transition probabilities are assumed to be time-invariant (compare footnote 33). Since switching from state $h$ can only take place to one of the $M$ states, the rows of $\boldsymbol{\Gamma}$ sum up to unity by construction, i.e., $\boldsymbol{\Gamma} \mathbf{1}_{M}=\mathbf{1}_{M}$ where $\mathbf{1}_{M}=(11 \ldots 1)^{\top}$ is a $(1 \times M)$ vector, which is equivalent to $\sum_{j=1}^{M} \gamma_{h j}=1, h=1, \ldots, M$. The state process $\left\{J_{t}\right\}$ determined by the transition probabilities $\gamma_{h j}$ can thus be modeled quite flexibly. For example, the larger the probability on the diagonal of $\boldsymbol{\Gamma}$ of some state is, the more persistent the behavior of this state will appear and the less switches from this state to others will occur on average.

Several assumptions on the properties of the Markov chain have to be made in order 
to keep the model in a tractable complexity and to ensure desirable properties of the time series and the regimes. The RGP is assumed to satisfy the Markov property:

$$
\operatorname{Pr}\left(J_{t+1} \mid J_{t}, J_{t-1}, \ldots, \boldsymbol{p}_{t}, \boldsymbol{p}_{t-1}, \ldots\right)=\operatorname{Pr}\left(J_{t+1} \mid J_{t}\right)
$$

which is also referred to as a first-order or a memoryless process. This property states that the probability of switching to a new state in $t+1$ solely depends on the state of the preceding period $t$ or as Chung (1960) puts it, "the past should have no influence on the future except through the present". Neither states before $J_{t}$ nor any further variables such as the observed price series contain additional information regarding the regime switching. This assumption is not restrictive since each more complex model can be reparametrized into a first-order model; see, for example, Hamilton (1994, ch. 22.4) or MacDonald and Zucchini (1997, ch. 1.3). Moreover, the Markov chain has to be assumed to be ergodic and irreducible. The first condition is necessary to ensure a stationary unconditional probability distribution of the regimes. ${ }^{36}$ The second one is needed to ensure the stationarity of the resulting time series. It requires that the ergodic probabilities of all states are larger than zero. Hence, it is assumed that any state can be reached from any state, that is that there are no absorbing states.

Figure D.4 depicts the transition graph of a Markov chain of trade with $M=2$ states. It displays the possibilities for switching between two subsequent periods and the associated transition probabilities, i.e., it illustrates the information contained in the transition matrix $\boldsymbol{\Gamma}$. In state $j=1$, trade is not inhibited by, for example, governmental measures; in state $j=2$ it is. The realization of a MSVECM in Figure

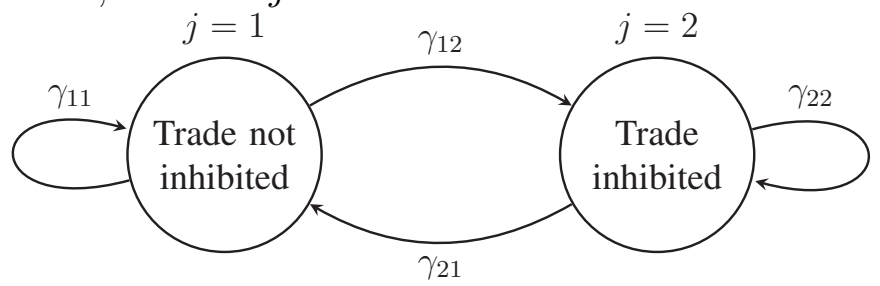

Figure D.4: Transition Graph of a Two-State Markov Chain

D.5 is generated according to (D.13) and corresponds to the Markov chain in Figure D.4. If, say, the Markov chain is at $t=0$ in state $J_{0}=2$, as depicted in the upper panel of the figure, the loading parameters $\boldsymbol{\alpha}^{\left(J_{t}\right)}$ take the values $\boldsymbol{\alpha}^{(2)}$, i.e., the correction of deviations from the long-run equilibrium in this period takes place with a high magnitude of $\mathrm{PT}$ of \pm 0.25 . The switching to the state in the next time period $t=1$ solely depends on the previous state and the respective transition probabilities (Markov property). For $J_{0}=2$ the state $J_{1}$ of the following period is generated by a random switch based on the probabilities $\gamma_{22}=0.8$ and $\gamma_{21}=1-\gamma_{22}=0.2$. Following this mechanism, the state in $t=1$ will be, say, $J_{1}=2$. PT in this period is in turn characterized by the adjustment speeds $\boldsymbol{\alpha}^{(2)}$. These adjustment speeds will prevail

\footnotetext{
${ }^{36}$ The expected unconditional probabilities of the of being in any of the $M$ states at arbitrary time are called the ergodic probabilities of the chain. Hence, the empiric frequencies of the regimes asymptotically equal the ergodic probabilities.
} 
until the Markov chain switches to state $j=1$ at some time $t$ (the ninth time point in the figure).

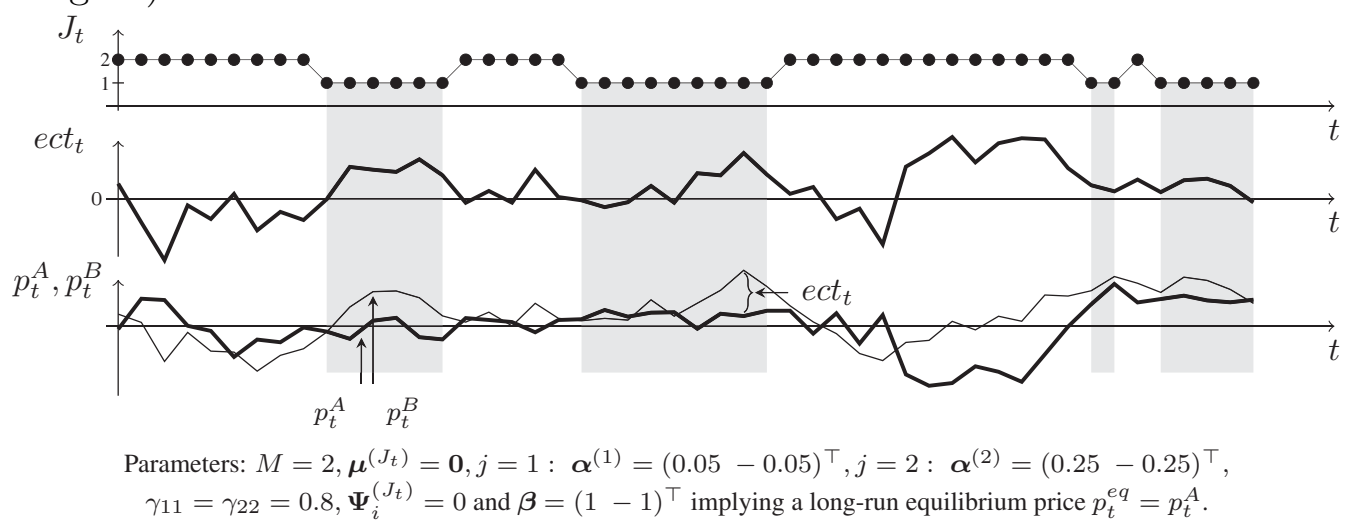

Figure D.5: Realization of a $\operatorname{MSVECM}(2)$

\section{Estimation}

In contrast to the estimation of the TVECM, one method is used for the estimation of the MSVECM as well as for general Markov-switching models in practice. ${ }^{37}$ The particular challenge for estimation is similar to the TVECM. The researcher encounters uncertainty on two levels. First, the state process $\left\{J_{t}\right\}$ depends on $\mathbf{A}^{(j)}$ in (D.9). It has to be estimated since it is unknown. Second, the model parameters $\mathbf{A}^{(j)}$ in turn depend on the unknown states $J_{t}$ and are also to be estimated. ${ }^{38}$

Due to this two-fold uncertainty, the estimation consists of two steps, the expectation step and the maximization step. These steps are reiterated and the inference about the states and the estimates is updated until some convergence criterion is met. The procedure is called the Expectation-Maximization algorithm (EMA) (Figure D.6). A particular filter is used in its first step, called the Baum-Lindgren-HamiltonKim (BLHK) filter. ${ }^{39}$ The EMA was introduced by Dempster, Laird, and Rubin in 1977. Hamilton (1990) proposes the usage of the BLHK filter in connection with the EMA. Kim (1994) contributes an important improvement of the expectation step. Krolzig (1997, ch. 6) provides a detailed account of the method, mentioning its major advantages of computational simplicity and desirable convergence properties, and discussing various extensions. The algorithm is initialized by assuming starting values for the model parameters, the transition matrix and the probabilities of being in each of the $M$ regimes at $t=1$. The following expectation step draws inference about the

\footnotetext{
${ }^{37}$ Krolzig (1997, ch. 8) outlines an alternative estimation method (multi-move Gibbs sampling), which is based on Bayesian statistics. Mizrach and Watkins (2000) mention hill climbing. However, they recommend the EMA because of its superior properties.

${ }^{38}$ Hamilton (1990) mentions three problems of interest to the researcher: the inference about the unobserved regimes of the sample; the conditional forecast; and the estimation of the model parameters, including the transition matrix.

${ }^{39}$ For more details, compare Krolzig (1997, ch. 5).
} 


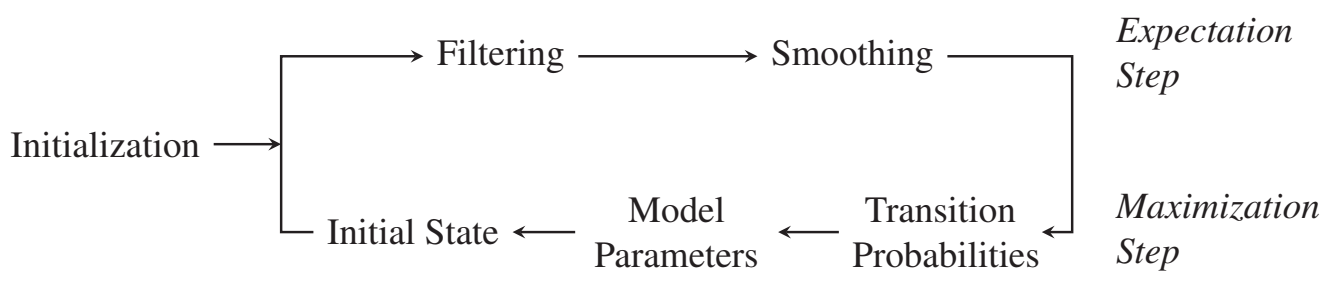

Figure D.6: The Expectation-Maximization Algorithm

unobserved regimes. First, the observations are filtered with the BHLK filter which yields the filtered probabilities. These are the probabilities that the observation at time $t$ has been generated by each of the $M$ regimes conditional on the data up to $t$ and the estimated model parameters which are, in the case of the first iteration, the initially assumed ones. Afterwards, the full sample smoothed probabilities are obtained on the basis of the filtered probabilities by a backward recursion. They represent the probabilities for each of the $M$ regimes that it has occurred at time $t$ conditional on the entire sample at hand. Equivalently, they may be interpreted as the probabilities that the observation at time $t$ has been generated by regime $j$ conditional on the entire sample.

The maximization step computes the update of the maximum likelihood estimates of all parameters, which include the transition probabilities, the vector error-correction parameters, and the probabilities of being in each of the $M$ regimes at $t=1$, that is, the initial state. The transition probabilities $\gamma_{h j}$ are updated as the ratio between the summed probabilities of switches from $h$ to $j$ and of occurrences of regime $h$ throughout the sample. Both quantities are calculated on the basis of the smoothed probabilities from the performed expectation step. The regime-dependent vector error-correction parameters $\mathbf{A}^{(j)}$ are calculated via generalized least squares estimation in which the observations are weighted by their smoothed probabilities. The second step finishes with the update of the probabilities of being in each of the $M$ regimes at $t=1$ which are estimated by the smoothed probabilities for $t=1$. The first iteration has thus been completed. The second iteration starts with utilizing the updated parameters from the previous one for the calculations in the expectation step where inference about the states is updated again. The second iteration is then completed by the update of the parameter estimates in the maximization step. The third iteration starts, and so on, until some reasonable convergence criterion is met.

This algorithm works for the estimation of Markov-Switching models in general. For the MSVECM in particular, Krolzig (1996) recommends a two-step estimation where first the cointegration vector and the equilibrium errors are obtained. The equilibrium error may then be treated as an exogenous regressor in the model, which becomes a general MSVAR model. The EMA can then be applied to the latter as described above. 


\section{Interpretation}

In the case of the MSVECM, the inference on the regimes is of probabilistic nature. Hamilton and Raj (2002b) note that there is a "growing consensus among economists that regime changes might be more appropriately modeled as arising from a probability process such as the Markov process". The RGP is assumed to follow a latent Markov chain. Hence, the researcher cannot say with certainty which regime has occurred at some time $t$. The only measures allowing inference on this question are the smoothed probabilities which lie between zero and one. The regime with the highest smoothed probability for some time $t$ is most likely to occur.

The interpretation of the regimes is far from being obvious a priori. It is much less straightforward than it is for the TVECM. The Markov-switching methodology is capable of identifying distinct regimes among the observations of the sample. However, it relies exclusively on the sample by doing so. Hence, it is the researcher's task to make sense of the identified regimes since no immediate interpretation based on economic theory is available as in, e.g., the case of Heckscher's supposition for the TVECM. The regimes have to be thoroughly analyzed and contrasted. Furthermore, an instructive endeavor might be to hypothesize the number and timing of regimes, or at least potential determinants, before performing the econometric analysis. By carefully analyzing potentially relevant events in the political and economic environment during the sample period, insights into the dynamics of the markets under study may be gained. The data analysis might then be used less as an exploratory but rather as a confirmatory tool. Jackman (1995) discusses the danger of the ex post "labeling of states". He argues that a thorough interpretation of the estimates of each regime is necessary for characterizing the detected states. Alternatively, one might try to impose some structure on the Markov process or approach the issue from a Bayesian point of view by incorporating prior knowledge. ${ }^{40}$

The MSVECM allows, in a similar way that the TVECM does, not only for regimes characterized by different speeds of error adjustment but also for periods where no error-correction takes place, as, for example, in Psaradakis et al. (2004a). The latter case is particularly interesting. In contrast to the TVECM, such a regime is not bounded; the longer the regime prevails, the farer the prices, which are not cointegrated in this regime, may wander away from the equilibrium relationship. Such random walk behavior leads to high deviations from equilibrium which are not corrected despite their magnitude. Thus, such a regime might be interpreted as being characterized by prohibitive transaction costs which do not allow for any trade although deviations from equilibrium might become huge. Such a extreme regime of PT might, for example, be caused by political intervention or other forms of prohibitive trade barriers which either lead to immense costs of trade or do not allow for trade at all. Consequently, the MSVECM may be seen as being able to detect temporarily changing transaction costs where the change takes place in the form of discrete shifts.

\footnotetext{
${ }^{40}$ He provides a comprehensive and detailed example of the first approach in his article.
} 


\section{Comparison}

\subsection{Conceptual Comparison}

The application of TAR models to PT analysis is appropriate in general due to the regime-dependent behavior of $\mathrm{PT}$ as depicted in Figure D.2. The application of the TVECM in particular, with Heckscher's supposition and the spatial arbitrage models of Takayama and Judge, has immediate economic justification; the MSVECM only has justification to a limited extent, mainly due to the lack of attention it has attracted as of yet in the field. Nevertheless, the application of the latter model in PT analysis seems intuitively very reasonable, particularly in cases where "discrete shifts in regime-episodes" (Hamilton, 1989) seem to be present in the data and the trade process was "occasionally disrupted by dramatic events" (Hamilton and Raj, 2002b) or "a sudden shock" (Psaradakis et al., 2004a).

Both models can be formulated in terms of (D.9) which represents a special case of the general threshold model specification in (D.2). Although both approaches model regime-dependent behavior of time series and belong to the group of piecewise linear TAR models (Figure D.1), the philosophy regarding their underlying RGPs differs fundamentally. This leads to differing estimation methods and interpretation of results.

In case of the TVECM, the regime process $\left\{J_{t}\right\}$ is assumed to be exclusively generated by the first lag of some linear combination of the two price series under investigation, i.e., $J_{t}=f\left(p_{t-1}^{A}, p_{t-1}^{B}\right)$. In the case of the MSVECM, it is assumed rather to be a function of one or more exogenous variables $y, z, \ldots$ which might be thought of as the "general state" of the system $J_{t}=f\left(y_{t}, y_{t-1}, \ldots, y_{t-l}, z_{t}, z_{t-1}, \ldots, z_{t-r}, \ldots\right)$ where $l, r \in$. In contrast to the former model, the state process is allowed to be latent. Consequently, no observations on the regime generating variable(s) are required; they may even stay entirely unspecified. In this light, the assumption of the TVECM that the equilibrium error $e c t_{t}$ is the only variable determining the regimes seems restrictive. However, if the time series to be analyzed emerged in a stable economic and political environment in the absence of abrupt changes and other events which are likely to influence trade, the TVECM is the more appropriate model. It implies that there are at least two regimes in the data, even three regimes in the case of trade reversals, and that the deviations from the long-run equilibrium ect $t_{t}$ is the only variable causing regime switching.

In the case of the existence of only one spatial equilibrium condition in the data or a highly unstable political and/or economic environment, in which trade as one aspect of the economy is embedded, regimes of PT are not likely to be (exclusively) determined by the equilibrium error. Regime shifts due to exogenous factors may superimpose the (weak) regimes created by spatial equilibrium conditions and dominate the RGP. Con- 
sequently, ect $t_{t}$ does not represent the variable causing regime shifts. The MSVECM would be more appropriate in such a case. Consequently, the assumption that the equilibrium error represents the only variable causing nonlinear PT might not reflect reality in some settings.

These diverging suppositions regarding the RGP entail differing inference concerning the regime incidences. Whereas statements about the regime occurred at some time $t$ can be made with certainty for the TVECM, they can only be of probabilistic nature in the case of the MSVECM. Allowing for non-deterministic statements regarding the regime incidences turns out to be a reasonable and justified approach. Trade as well as business cycles or presidential approval are highly complex processes generated by unknown dynamics which are likely to be of nonlinear character. Although the methodology is capable of detecting evidence in the data at hand that some observations are likely to follow a different regime, the researcher can, of course, not completely be sure about such a finding because the true RGP remains unknown. This remaining uncertainty is acknowledged by considering probabilistic statements regarding the occurrence of the regimes as the MSVECM does.

In the case of the TVECM, the statements about the occurrence of regimes are deterministic in the sense that a certain regime $j$ has or has not occurred at time $t$ with certainty, i.e., the point estimates of $e t_{t}$ can uniquely be assigned to the $l$ regimes of the model which is implicitly formulated in (D.9). The binary variable $\mathbf{d}_{t}^{(j)}$ takes the value 1 if the regime $j$ occurs at time $t$ or zero otherwise. Clearly, such a deterministic all-or-nothing statement is more restrictive than the corresponding probabilistic statement of the MSVECM; however, on the other hand, it allows for an easier interpretation. Whereas the Markov-switching approach acknowledges the uncertainty concerning the unknown true DGP, the TVECM approach does not. It instead suggests that whenever the estimated threshold variable falls into a certain interval, the corresponding regime prevails with certainty. This implication is quite strong and may, of course, not be true for all observations. This assignment may have occurred occasionally by chance instead of being caused by the supposed underlying RGP.

Both models are capable of detecting regimes characterized by different rates of errorcorrection as well as regimes in which no adjustment behavior takes place. Although the TVECM does not explicitly model transaction costs, the threshold estimates are, at least in the case of a TVECM(3) specification, usually interpreted as such. Moreover, they are often assumed to be constant during the sample period. The MSVECM also does not model transaction costs. Nevertheless, it might be understood as to allow the transaction costs to shift during the sample period since an identified regime without adjustment may potentially be caused by temporary prohibitive transaction costs.

The estimation of both models faces the same challenge. The parameters of the regimedependent VECM are unknown and depend on the regime process $\left\{J_{t}\right\}$. This process itself is unknown because the quantities characterizing it, which are the thresholds 
and the transition probabilities, respectively, are unknown as well. Their estimates in turn depend on the unknown vector error-correction parameters. The estimation methods of SCLS and EMA, though variants of the maximum likelihood principle, tackle this task in different ways. In the former case, a number of modifications are easily implemented to estimate the model in dependence of various optimization parameters (Table D.1). The researcher determines candidate values of the optimization parameters which typically form a regular spaced grid. Conditionally on these, an optimization criterion is evaluated. The combination of parameters optimizing the criterion is selected as the final estimates. The EMA, in contrast, iterates conditionally on one set of starting values until a convergence criterion is met. Inference on the unobserved regimes is recursively drawn for all observations conditional on the parameter estimates of the previous iteration. Parameter estimates, in turn, are obtained conditionally on the evidence on the regimes from the preceding step. In the case of both methods, the number of regimes may either be justified theoretically, evaluated by econometric tests, or determined by using a model selection criterion. ${ }^{41}$

Differences between both methods concerning the interpretation have already been addressed. Of course, regime frequencies and regime-dependent half-lives of the adjustment process may be calculated in both cases. Additionally, the expected duration of the regimes may be calculated for the MSVECM. The regime frequency is estimated by the proportion of observations generated by the regime. In the case of the MSVECM it is the proportion of observations which is likely to be generated by the regime, wherein the meaning of the term "likely" has to be determined by the researcher. The regime with the highest smoothed probability among all regimes for some time $t$ is considered to be the most likely. The half-life of an adjustment process is the time which is required to correct half of the deviation from equilibrium of a given shock (van Campenhout, 2007) and can easily be obtained. ${ }^{42}$ However, the calculation of half-lives is more complicated for vector autoregressions of higher order, as pointed out by Ben-Kaabia and Gil (2007). The expected duration $\lambda^{j}$ of regime $j$ can be calculated as $\lambda^{j}=\mathrm{E}\left[\lambda \mid J_{t}=j\right]=\frac{1}{1-\gamma_{j j}}$ as outlined in Krolzig (1997, subsection 11.3.4). $\gamma_{j j}$ denotes the transition probability of staying in regime $j$ as depicted in the transition matrix $\Gamma$ (D.15).

Furthermore, it has been noted that the interpretation of the regimes of the TVECM

\footnotetext{
${ }^{41}$ In this paper, we address neither the issue of testing for nonlinearity nor that of impulse response analysis, since it is beyond its scope. However, we will briefly discuss the issue of model selection below.

${ }^{42}$ The half-life $\kappa$ is the solution in $z_{t+\kappa}=\frac{z_{t}}{2}$ based on a SETAR specification of the equilibrium error process, as, for example, in Balke and Fomby (1997, equation (1)). As they have shown, the SETAR and the TVECM specification are equivalent; the former represents a reparametrization of the latter and vice versa. Hence, the half-life is calculated as $\kappa=\frac{\ln (0.5)}{\ln \left(1+\rho^{(j)}\right)}$ based on the SETAR specification. In the TVECM specification, $\rho^{(j)}$ is not estimated. It has thus to be replaced by $\rho^{(j)}=1+\boldsymbol{\beta}^{\top} \boldsymbol{\alpha}^{(j)}=1+\boldsymbol{\alpha}^{A(j)}-\beta^{B} \boldsymbol{\alpha}^{B(j)}$ where $\boldsymbol{\alpha}^{A(j)}$ denotes the magnitude of PT of the $j$ 's regime of the price series of market $A$ and $\boldsymbol{\beta}=\left(\beta^{A} \beta^{B}\right)^{\top}=\left(1 \beta^{B}\right)^{\top}$ the cointegration vector between both prices.
} 
is relatively straightforward. However, the results are occasionally interpreted in a narrower or broader sense. In the case of the MSVECM, some effort has to be devoted to carefully analyzing the identified regimes. The parameters and further descriptive variables have to be interpreted and consulted in detail in order to receive insights regarding the distinguishing characteristics and the nature of the regimes.

\section{Distinct Regime Generating Processes: Exogenous vs. Endogenous Switching}

As mentioned above, both models may be formulated in terms of the general specification (D.10). Nevertheless, the RGPs differ fundamentally. A formulation in terms of a common notation permits insights from one perspective regarding their similarities and differences. In particular, the RGP of the TVECM can be reformulated by using the notation of the MSVECM. The key distinction in the philosophies underlying both approaches becomes apparent and can well be contrasted by using a unified notation. In the following, we restrict the comparison to the simplest specification of $l+1=M=2$ regimes for each of the models.

The transition matrix of a MSVECM with $M=2$ regimes has the following structure

$$
\boldsymbol{\Gamma}=\left(\begin{array}{ll}
\gamma_{11} & \gamma_{12} \\
\gamma_{21} & \gamma_{22}
\end{array}\right)=\left(\begin{array}{cc}
\gamma_{11} & 1-\gamma_{11} \\
1-\gamma_{22} & \gamma_{22}
\end{array}\right)
$$

because $\boldsymbol{\Gamma} \mathbf{1}_{M}=\mathbf{1}_{M}$. Thus, the corresponding Markov chain is characterized by only two transition probabilities. It is assumed to be homogeneous, that is, the transition probabilities are assumed to be constant. Moreover, for the transition probabilities it is assumed to hold that $0<\gamma_{11}<1$ and $0<\gamma_{22}<1$ so that the chain is irreducible. The process is assumed to satisfy the Markov property. Alternatively, the transition matrix can be rewritten in terms of a set of conditional probabilities

$$
\begin{aligned}
& \operatorname{Pr}\left(J_{t}=1 \mid J_{t-1}=1\right)=\gamma_{11} \\
& \operatorname{Pr}\left(J_{t}=1 \mid J_{t-1}=2\right)=1-\gamma_{22} \\
& \operatorname{Pr}\left(J_{t}=2 \mid J_{t-1}=1\right)=1-\gamma_{11} \\
& \operatorname{Pr}\left(J_{t}=2 \mid J_{t-1}=2\right)=\gamma_{22} .
\end{aligned}
$$

A TVECM of $l+1=2$ regimes possesses one (effective) threshold $\theta^{(1)}$. The RGP is characterized as implicitly formulated in (D.6)

$$
J_{t}= \begin{cases}1 & \text { if ect } \\ 2 & \text { if } \text { ect }_{t-1}>\theta^{(1)}\end{cases}
$$

As mentioned above, $J_{t}$ takes the values $j$ with certainty. Depending on the size of the threshold variable which is in this case the first lag of the error-correction term ect $t_{t-1}$, the regime $j$ prevails with a probability of $100 \%$ at time $t$. Hence, it becomes evident 
that the RGP may be formulated in terms of conditional probabilities which can be summarized into a transition matrix. However, due to the mentioned certainty, these probabilities take either of the values 0 or 1 . They are conditional on the previous state $J_{t-1}$, however, they additionally depend on the treshold variable $e t_{t-1}$ of the previous period. The corresponding transition probabilities are as follows

$$
\begin{array}{cc}
\text { for } \text { ect }_{t-1} \leq \theta^{(j)} & \text { for ect } t_{t-1}>\theta^{(j)} \\
\operatorname{Pr}\left(J_{t}=1 \mid J_{t-1}=1, \text { ect }_{t-1}\right)=\omega_{11}^{(1)}=1 & \operatorname{Pr}\left(J_{t}=1 \mid J_{t-1}=1, \text { ect }_{t-1}\right)=\omega_{11}^{(2)}=0 \\
\operatorname{Pr}\left(J_{t}=1 \mid J_{t-1}=2, \text { ect }_{t-1}\right)=\omega_{21}^{(1)}=1 & \operatorname{Pr}\left(J_{t}=1 \mid J_{t-1}=2, \text { ect }_{t-1}\right)=\omega_{21}^{(2)}=0 \\
\operatorname{Pr}\left(J_{t}=2 \mid J_{t-1}=1, \text { ect }_{t-1}\right)=\omega_{12}^{(1)}=0 & \operatorname{Pr}\left(J_{t}=2 \mid J_{t-1}=1, \text { ect }_{t-1}\right)=\omega_{12}^{(2)}=1 \\
\operatorname{Pr}\left(J_{t}=2 \mid J_{t-1}=2, \text { ect }_{t-1}\right)=\omega_{22}^{(1)}=0 & \operatorname{Pr}\left(J_{t}=2 \mid J_{t-1}=2, \text { ect }_{t-1}\right)=\omega_{22}^{(2)}=1 .
\end{array}
$$

These probabilities can be summarized into the following transition matrix $\Omega$, which depends on the threshold variable $e c t_{t-1}$

$$
\boldsymbol{\Omega}=\boldsymbol{\Omega}_{t}=\left\{\begin{array}{l}
\boldsymbol{\Omega}^{(1)}=\left(\begin{array}{ll}
\omega_{11}^{(1)} & \omega_{12}^{(1)} \\
\omega_{21}^{(1)} & \omega_{22}^{(1)}
\end{array}\right)=\left(\begin{array}{ll}
1 & 0 \\
1 & 0
\end{array}\right) \quad \text { if ect } \text { t }_{t-1} \leq \theta^{(1)} \\
\boldsymbol{\Omega}^{(2)}=\left(\begin{array}{ll}
\omega_{11}^{(2)} & \omega_{12}^{(2)} \\
\omega_{21}^{(2)} & \omega_{22}^{(2)}
\end{array}\right)=\left(\begin{array}{ll}
0 & 1 \\
0 & 1
\end{array}\right) \quad \text { if ect } \text { en }_{t-1}>\theta^{(1)} .
\end{array}\right.
$$

This transition matrix $\Omega$ is equivalent to the usual specification of the RGP of a TVECM, e.g., as formulated in (D.22). It highlights the similarities and the differences of the TVECM in comparison to the transition matrix $\boldsymbol{\Gamma}$ of the MSVECM. In the case that the error-correction term is smaller than the threshold, it does not matter which state the process was in during the preceding period $t-1$ it takes the regime $j=1$ in time $t$ with probability 1 . This regime is either reached by staying in the regime 1 , which is expressed by $\omega_{11}^{(1)}$, or by switching from regime 2 to one 1 , expressed by $\omega_{21}^{(1)}$. In the case that the error-correction term is larger than the threshold, the process will be in regime 2 at time $t$ with probability 1 .

It becomes apparent that the transition matrix $\Omega$ is not constant over time because the respective transition probabilities take values depending on the magnitude of the error-correction term. Thus, the matrix symbol is augmented by the time index $t$ and denoted as $\Omega_{t}$. Consequently, this RGP, in contrast to the MSVECM in (D.17), is not homogeneous. Second, the transition probabilities are restricted to the values 0 or 1. In this sense, the switching is purely deterministic. It either occurs or it does not, either way with certainty.

Third, the process does not satisfy the Markov property because $\operatorname{Pr}\left(J_{t} \mid J_{t-1}\right.$, ect $\left._{t-1}\right) \neq$ $\operatorname{Pr}\left(J_{t} \mid J_{t-1}\right)$. It has been mentioned that the size of the error-correction term determines 
not only the regime but also the transition probabilities in $\Omega_{t}$. Hence, it contains additional information for the switching of the regimes. In contrast, the entire information relevant for switching is encompassed in the previous state in case of the MSVECM as denoted in (D.18) to (D.21). Moreover, the transition probabilities in $\boldsymbol{\Omega}_{t}$ do exclusively depend on the threshold variable because a state $j$ is reached at time $t$ from any preceding state with certainty, exclusively determined by the magnitude of the threshold variable. This view corresponds to the usual interpretation of the TVECM that the switching exclusively depends on the error-correction term. Hence, it holds that $\operatorname{Pr}\left(J_{t} \mid J_{t-1}, e^{e c t_{t-1}}\right)=\operatorname{Pr}\left(J_{t} \mid\right.$ ect $\left._{t-1}\right)$ and (D.23) to (D.26) and the transition matrix $\Omega_{t}$ simplify to

$$
\Omega_{t}^{\prime}=\left(\begin{array}{ll}
\omega_{11}^{\prime} & \omega_{12}^{\prime} \\
\omega_{21}^{\prime} & \omega_{22}^{\prime}
\end{array}\right)=\left(\begin{array}{ll}
1 & 0 \\
0 & 1
\end{array}\right)
$$

where $\omega_{1 j}^{\prime}=\operatorname{Pr}\left(J_{t}=j \mid e c t_{t-1} \leq \theta^{(1)}\right)$ and $\omega_{2 j}^{\prime}=\operatorname{Pr}\left(J_{t}=j \mid e c t_{t-1}>\theta^{(1)}\right), j=1,2$. This formulation emphasizes the fact that the switching is exclusively governed by the observed threshold variable in a deterministic way, whereas, in the case of the MSVECM, the switching is governed by the unobserved previous state in a probabilistic way as formulated in (D.17) to (D.21). Furthermore, in the case of the TVECM, the threshold variable is a linear combination of the two price series under study.

Consequently, the regime switching of the TVECM, in contrast to the MSVECM, is not exogenous. It has been shown that the probabilities $\omega_{h j}^{\prime}, h, j=1,2$ for switching to regime $j$ from time $t-1$ to time $t$ depend exclusively on the error-correction term $e c t_{t-1}$. This threshold variable itself is a function of the observed price series $\left\{p_{t}^{A}\right\}$ and $\left\{p_{t}^{B}\right\}$ of markets $A$ and $B$ because it represents a linear combination of the first lags of both series ect $t_{t-1}=\boldsymbol{\beta}^{\top} \mathbf{p}_{t-1}$. Hence, the transition probabilities are also a function of the two price series $\omega_{h j}^{\prime}=f\left(e^{\prime} t_{t-1}\right)=f\left(p_{t-1}^{A}, p_{t-1}^{B}\right)$ and the switching is thus endogenous. The switching of the MSVECM is exogenous since the variable causing the switching remains unknown.

\section{Model Selection}

The characterization of either of the considered approaches has been motivated in the previous sections by economic theory and heuristic evidence. To our knowledge, no econometric tests exist which explicitly test nonlinear model classes against each other. Mellows (1999) notes that this constitutes a common problem in nonlinear time series analysis. Although a number of tests have been developed to check for nonlinear behavior such as Hansen (1997), Hansen (1999) or Hansen and Seo (2002) for TAR or Hansen (1992) for Markov-switching models, the determination of the most appropriate model class for the data at hand remains an issue for future research. Mellows (1999, ch. 5) suggests a classification method based on a idea of Tong's (1990) which uses parametric bootstrap and discriminant analysis. However, a simulation study reveals that the more pronounced the specific nonlinearities of the underlying 
process are, the more likely a model class will be identified correctly by this approach. Under weak nonlinearities, one quarter to more than half of the models are wrongly classified as linear. However, diagnostic tests, as developed in Hamilton (1996) for example, help to assess the adequacy of the chosen model.

Considerations of testing one model against another may not be of immediate interest in the context of applied research in price transmission analysis since the application of a certain model class has to go along with an appropriate interpretation of results. Qualitative reasoning of the appropriateness of the chosen model accompanied by formal tests of nonlinearities in the time series and a thorough interpretation of estimation results may instead be a recommendable approach to tackle this issue.

Besides the question of which nonlinear model to choose, the question of whether nonlinear models are superior to linear time series models has to be addressed. It has been discussed above that both models considered here possess much appeal from an economic perspective. Clements and Krolzig (1998) assess the performance of two nonlinear time series models in comparison with linear AR models in business cycle analysis. They find that although both Markov-switching autoregressive and SETAR models are well capable to model the particular features of the data, their performance in forecasting is not superior to AR models. This question is not discussed for the models considered in this paper. However, more complex models are in general more capable to capture the distinctive features of the data. In contrast, their forecasting performance does not necessarily increase due to their complexity. This general fact should also hold for the TVECM and the MSVECM.

\subsection{A Simulation Study}

In addition to the conceptual comparison of the TVECM and the MSVECM, we are interested in assessing the performance of the estimation methods under ideal circumstances. In particular, we apply the sequential conditional least squares (SCLS) and the Expectation-Maximization-Algorithm (EMA) to simulated data which is generated by a TVECM and a MSVECM respectively. ${ }^{43}$ Problems of the SCLS estimation have rarely been addressed in applied research. Lo and Zivot (2001) study the performance of the method for data generated according to a TAR and a TVECM for symmetric thresholds of 3,5, and 10, and time series of 100, 250, and 500 observations, respectively. They find "considerable uncertainty in the estimates [of the thresholds] for moderate sample sizes" in the case of the unrestricted model. However, the estimates of restricted TAR and TVECM models have a much smaller bias and also a smaller variance. Furthermore, Trenkler and Wolf (2003) note that the estimates of

\footnotetext{
${ }^{43}$ The Bayesian approaches of Luoma et al. (2004) and Balcombe et al. (2007) for estimating the TVECM are not considered here. We focus on SCLS and EMA since they are the predominant estimation methods in applied research in PT.
} 
the unrestricted TVECM are very unreliable.

With this simulation study we extend the work of Clements and Krolzig (1998), Lo and Zivot (2001) and Psaradakis et al. (2004b). The first article assesses the forecast performance of Markov-switching and TAR models relative to linear autoregressive models in business cycle analysis via a simulation study. Lo and Zivot study the performance of tests for threshold cointegration, threshold nonlinearity, and specification tests; they evaluate the estimation of the TVECM by an extensive simulation study. Psaradakis et al. (2004b) examine tests for cointegration, parameter instability, neglected nonlinearity, Markov-switching, and a model selection procedure based on data which follows Markov-switching error-correction.

We follow Lo and Zivot (2001) by generating data according the simple cointegration model as outlined in Balke and Fomby (1997). ${ }^{44}$ Lo and Zivot generate data with varying lengths of the time series and magnitudes of the true thresholds which they assume to be symmetric. However, we adopt a more comprehensive setting by generating time series of length 150, 500 and 1500, respectively. We vary the thresholds $\theta^{(j)}$, the transition probabilities $\gamma_{h j}$ and the error-correction parameters $\boldsymbol{\alpha}^{(j)}$. The data is generated according to a TVECM and a MSVECM respectively. Each model is assumed to have three regimes, one of which does not show error-correction. In the following, we briefly present some results of the simulation study for $T=500$. Further results may be obtained from the authors.

\section{Case I: SCLS and TVECM Data}

A very low share, namely only around $56 \%$ of all observations, are correctly identified by this method. Hence, SCLS seems not to be able to identify the true regimes of TVECM-data as generated by ((DII.2)) to a satisfying extent. The reason lies in the very high and varying MSE as depicted for $\hat{\theta}^{(1)}$ in the following figure. The MSE is of considerable magnitude and strongly depends on the true thresholds $\theta^{(1)}, \theta^{(2)}$ as well as on the nuisance parameters $\rho^{(1)}, \rho^{(3)}$ which govern the autoregressive process of the ect $t_{t}$ as formulated in (DII.2). The bias is the smallest for $\left|\theta^{(j)}\right|=\sigma_{\nu_{t}}^{2}$. Hence, not only the magnitude of the thresholds themselves but rather the ratio between the absolute values of the thresholds and the variance of the innovations $\nu_{t}$ in (DII.2) seems also to have influence on the MSE. Both the bias and the variance tend to increase with decreasing $\alpha^{(j)}$ and with increasing $T$. This result is plausible since both, a small $\alpha^{(j)}$ and a large $T$, lead to more realizations of the data far away from the true thresholds and thus tend to increase bias and variance.

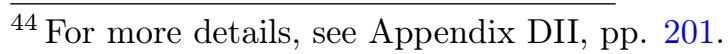



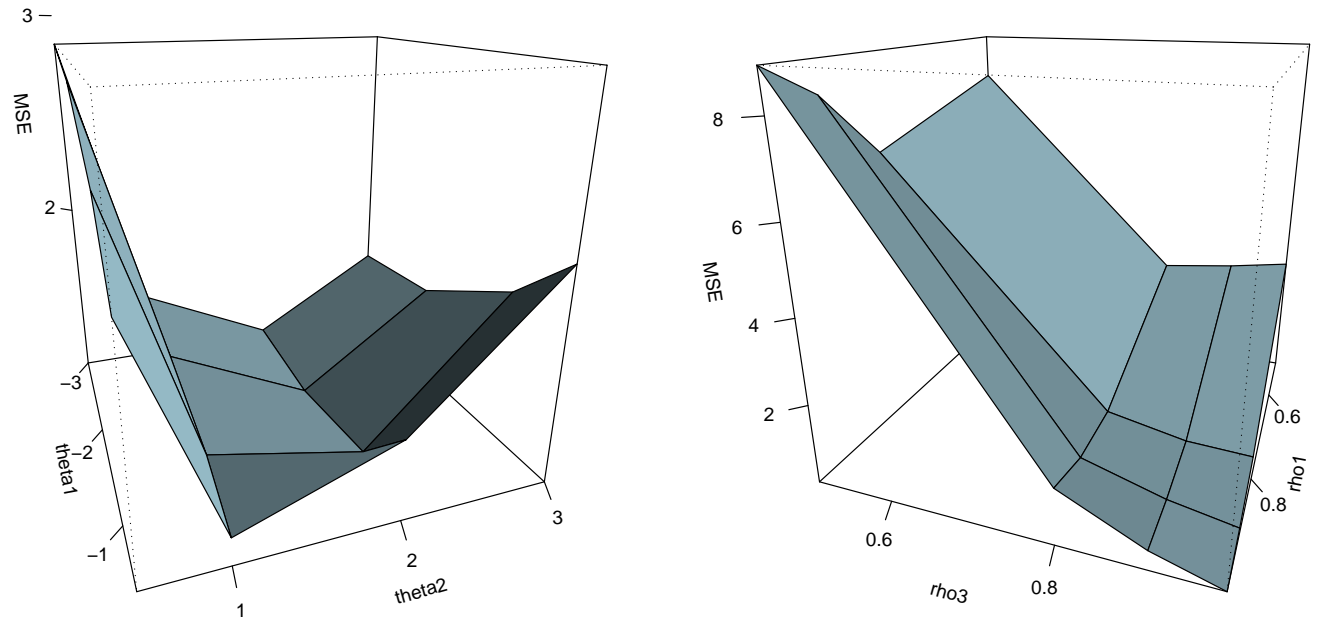

$$
\widehat{\operatorname{MSE}}_{\hat{\theta}^{(1)}}=f\left(\theta^{(1)}, \theta^{(2)}\right) \text { vs. } \widehat{\operatorname{MSE}}_{\hat{\theta}^{(1)}}=f\left(\rho^{(1)}, \rho^{(3)}\right)
$$

\section{Case II: SCLS and MSVECM Data}

The following table shows the classification of true vs. estimated regime incidences as percentages of all observations of the dataset. By summing up each of the columns, the ergodic probabilities of the MSVECM-data may be obtained. The percentages on the diagonal denote the correctly identified regimes, which sum up to only $40 \%$. SCLS performs even worse here than in case I. Hence, SCLS does not seem to be suitable to detect the true regimes in the data which is simulated by a MSVECM-DGP.

\begin{tabular}{l|lll}
\hline & R1 true & R2 true & R3 true \\
\hline R1 est & 0.339 & 0.076 & 0.041 \\
R2 est & 0.216 & 0.039 & 0.018 \\
R3 est & 0.214 & 0.039 & 0.019 \\
\hline
\end{tabular}

True vs. Estimated Regime Incidences in Case II

Case III: EMA and TVECM Data

The following table shows the classification of true vs. estimated regime incidences as estimated by the EMA on TVECM-data. A share of only about 30\% was correctly identified, indicating that EMA does not perform well with data that follow a TVECM.

\section{Case IV: EMA and MSVECM Data}

The following figure provides some evidence on the performance of the EMA on data that are generated by a MSVECM. The share of correctly identified regimes lies at about $42 \%$. Similarly to case I, the MSE of, say, the estimate of $\pi^{(1)}$, i.e., the ergodic 


\begin{tabular}{l|lll}
\hline & R1 true & R2 true & R3 true \\
\hline R1 est & 0.088 & 0.105 & 0.028 \\
R2 est & 0.133 & 0.152 & 0.044 \\
R3 est & 0.186 & 0.199 & 0.065 \\
\hline
\end{tabular}

True vs. Estimated Regime Incidences in Case III

probability of the first regime, is heavily influenced by the true ergodic probabilities as well as by the nuisance parameters $\rho^{(j)}$. Nevertheless, a strange pattern appeared in the estimation results. The bias for the first two regimes is very high in tendency and decreases as the true ergodic probabilities approach $\frac{1}{3}$ and increases with decreasing $\alpha^{(j)}$. The variance is small and increases slightly with increasing $\alpha^{(j)}$. Both the bias as well as the variance decrease with the increasing number of observations per time serioc
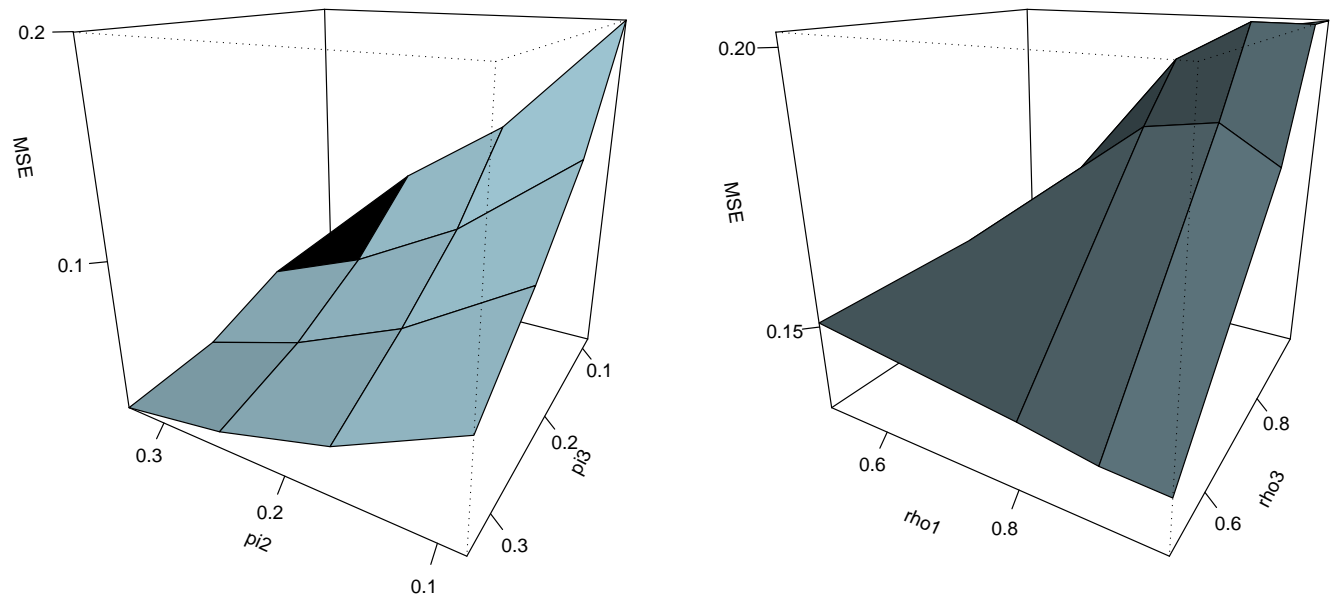

$$
\widehat{\operatorname{MSE}}_{\hat{\pi}^{(1)}}=f\left(\pi^{(2)}, \pi^{(3)}\right) \text { vs. } \widehat{\operatorname{MSE}}_{\hat{\pi}^{(1)}}=f\left(\rho^{(1)}, \rho^{(3)}\right)
$$

\section{Summary}

This Monte Carlo analysis leads to rather pessimistic results regarding the performance of the assessed estimation methods since only one third to one half of the regimes have correctly been identified. Lo and Zivot (2001) have demonstrated that the estimates of the thresholds are biased. We extended this evidence and demonstrated that the estimates of the thresholds also seem heavily influenced by the true error-correction parameters. The EMA did not yield satisfying results either. However, these results cannot be generalized since they depend on the assumptions made beforehand, particularly the specification of the DGPs. In order to derive general statements, further experiments have to be conducted. 


\section{Conclusion}

This paper compares two time series models which are relevant for PT analysis and allow for nonlinear adjustment of deviations from the long-run equilibrium. Both model classes, the TVECM as well as the MSVECM, are characterized by parameters which may take different values depending on the regime of the data. They are constant within one regime but may differ across regimes. Such regime-dependent models allow for the study of trade processes from a dynamic point of view in which the transmission of price signals between markets changes temporarily. Such sophisticated models may thus enhance the understanding of the interaction of markets. Although both models seem at first glance very similar due to their common property of regime switching, their underlying statistical concepts differ fundamentally. Consequently, each model is suited for a particular type of nonlinearity.

The TVECM is characterized by endogenous switching. The variable causing regime swit-ches is assumed to be fully determined by the prices under study. The restriction of the switching mechanism to a particular relationship facilitates the interpretation of the model, which matches economic theory very well. Such a constraint implies two aspects. First, if the explicit information contained in the threshold variable is correct, the model will yield more reliable results than more general ones. However, if the opposite is the case, then it will be further away from the truth than general models.

The MSVECM is more general with respect to the switching mechanism since it allows for exogenous switching independent of the price series analyzed. Furthermore, the determinants causing switching may even remain completely unspecified. Its key element is a latent Markov chain modeling the transition of regimes between subsequent points in time. The higher flexibility of the model comes at the cost of limited straightforward interpretability. Making sense of the identified regimes requires more effort than in the case of the former model.

The two models reflect different aspects of the complex economic reality: spatial equilibrium conditions in the one case and unobserved states of the system in the other. If the price data to be analyzed were predominantly not subject to external impacts such as changing political, economic or natural interferences, it can be assumed that markets and trade processes were the main forces generating the data. A TVECM would be the more appropriate model in such a case, since it explicitly draws on the economic information contained in the prices. Nevertheless, a TVECM requires at least two regimes in the data to be estimable. It requires, for example, changing spatial equilibrium conditions. If, however, trade mainly took place in one direction or external interferences dominated the markets during the time period studied, then a MSVECM might be more suitable. Most often, the reality will lie in between these two extreme cases. In such a case, the most appropriate model depends on the dominating impact. The two models can be expected to yield differing results since each 
emphasizes a certain aspect of PT.

Although both models, from an economic perspective, seem capable of leading to interesting insights into PT, the simulation study confirmed and extended evidence that the empiric application and the applicability constitutes a drawback of these models. However, improving the estimation of such models is not the only area for future research. Most often, the quantitative components of the theoretically postulated and econometrically estimated thresholds, i.e., the determinants causing nonlinear price adjustment, receive little attention. Acquiring empirical evidence regarding their structure might help to develop adapted models. As a consequence, the interpretation of the estimated thresholds only in terms of transaction costs may turn out to be too narrow in some cases. The magnitude of the time lag $d$ of the TVECM is likely to depend on the product traded, infrastructure, etc., so that by relaxing the assumption that prices react to deviations from equilibrium within a time lag of only one period, insights into the dynamic processes of trade might be extended. 


\section{References}

See references list of the dissertation, pp. 81 . 


\section{Appendix DI: Literature Review of Applications of the TVECM}

Table DI. 5 provides a review of studies of PT in commodity markets ${ }^{45}$ which are in most cases applications of the TVECM. We focus in the review on the data, models and estimation approaches used in the studies. It covers 35 publications of which 26 are journal articles. Tables DI.2 to DI.4 summarize the reviewed publications according to their publication type, research field and publication year.

\begin{tabular}{lccccc}
\hline Type & Journal art. & Confer. paper & Working paper & Dissertation & MSc thesis \\
Number & 26 & 4 & 3 & 1 & 1 \\
\hline
\end{tabular}

Table DI.2: Publications per Type

Table DI.5 lists the publications according to the initial letter of the first author's name. The first two columns give information on the type $T y$ of the article, where $j$ denotes "journal article", $c$ "conference paper", w "working paper", $d$ "dissertation" and $m$ "MSc thesis", and the field of research $F i$, where ae denotes "agricultural economics", eh "economic history", ee "energy economics" and $e$ "economics" in general. The following four columns provide information on the analyzed data. They outline the product(s) and the geographic region studied, their frequency $F r$, where $q$ denotes "quarterly", $m$ "monthly", $b$ "bi-weekly", w "weekly", $d$ "daily" and n.m. "not mentioned", and the length of the time series $T$ in number of periods.

\begin{tabular}{lcccc}
\hline Type & Agric. economics & Economic history & Economics & Energy economics \\
Number & 23 & 7 & 3 & 2 \\
\hline
\end{tabular}

Table DI.3: Publications per Research Field

The next five columns of Table DI.5 summarize features of the estimated model(s). The column Model classifies the model(s) used according to the discussion of equation (D.11), p.172, Reg denotes the number of regimes in the model; Cont indicates whether the model, if it has three regimes and is of the Band-type, is continuous. Sym indicates, if the model has three regimes, whether the model is symmetric; and $A d j$ states whether the model allows for a nonzero adjustment coefficient in the middle regime, i.e., whether $\boldsymbol{\alpha}^{(2)} \neq 0$.

\begin{tabular}{cccccccccccc}
\hline Year & 1997 & 1998 & 1999 & 2000 & 2001 & 2002 & 2003 & 2004 & 2005 & 2006 & 2007 \\
Num. & 1 & 2 & 1 & 2 & 2 & 3 & 2 & 3 & 7 & 5 & 7 \\
\hline
\end{tabular}

Table DI.4: Publications per Year

The last two columns provide information about the applied estimation method. Est outlines the (reference of) the estimation method applied where $H S$ denotes the method of Hansen and Seo (2002), B denotes "Bayesian estimation", LZ, LS, PT, OT, BF the method of Lo and Zivot (2001), "multivariate least squares", Prakash and Taylor (1997), Obstfeld and Taylor (1997) and Balke and Fomby (1997) respectively. Par denotes the parameters used in the optimization as defined in (D.6).

\footnotetext{
${ }^{45}$ Note that this literature review was prepared for a conference in April 2008. Consequently, it reflects the current literature only until approximately January 2008.
} 


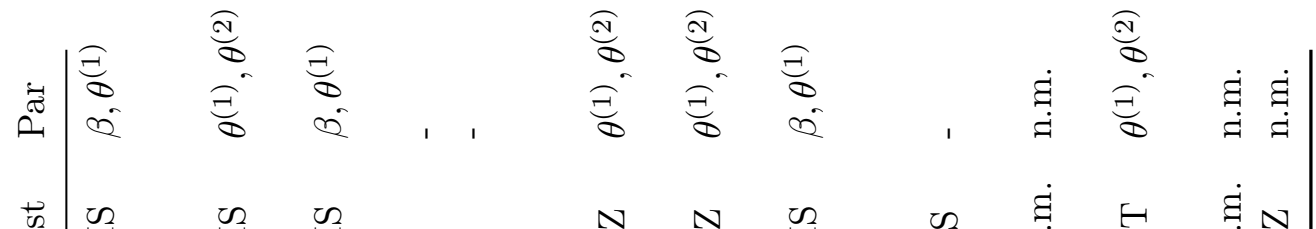

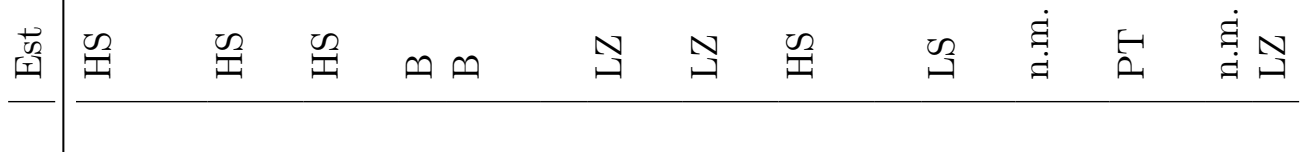

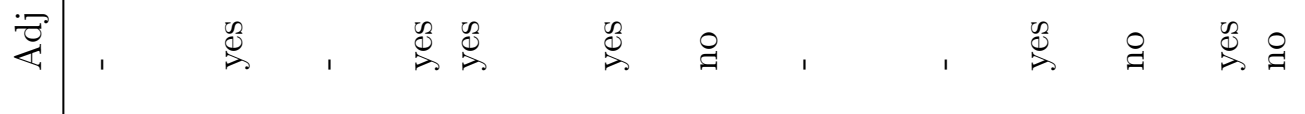

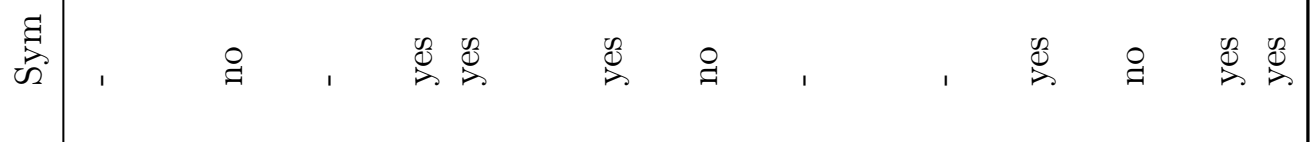

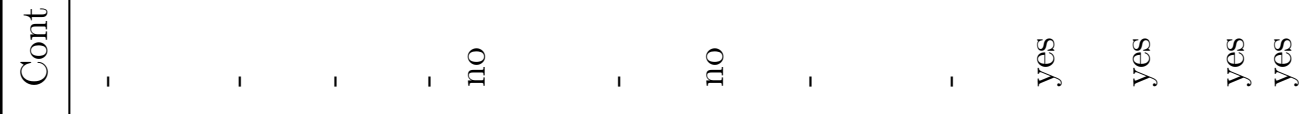

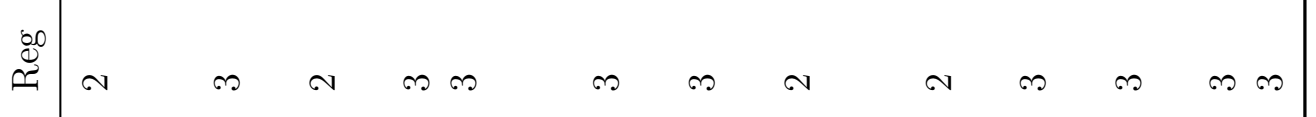

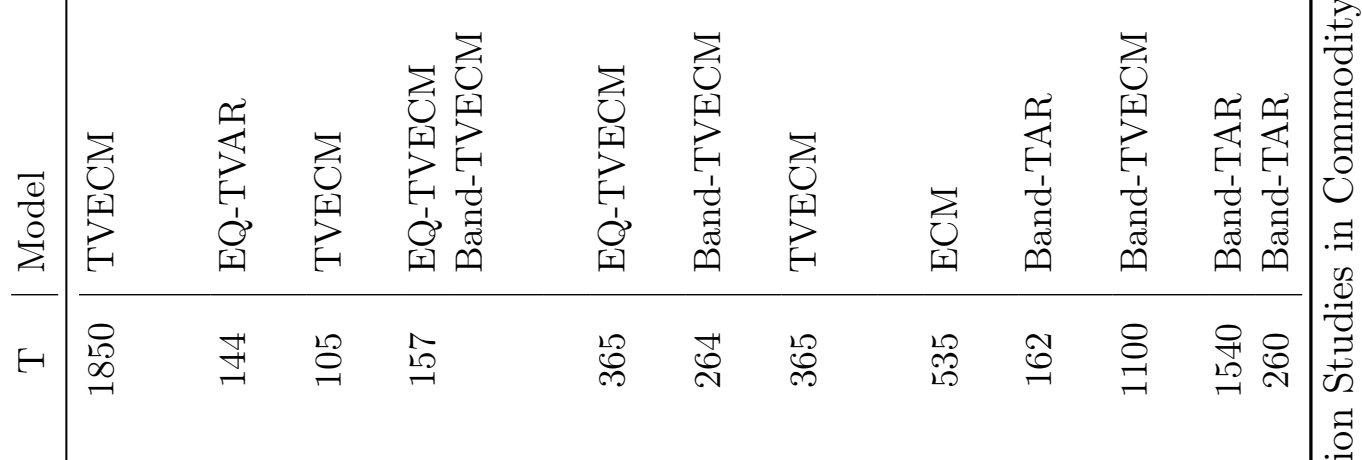

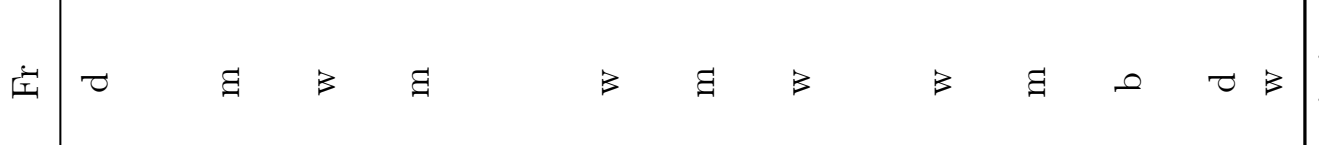

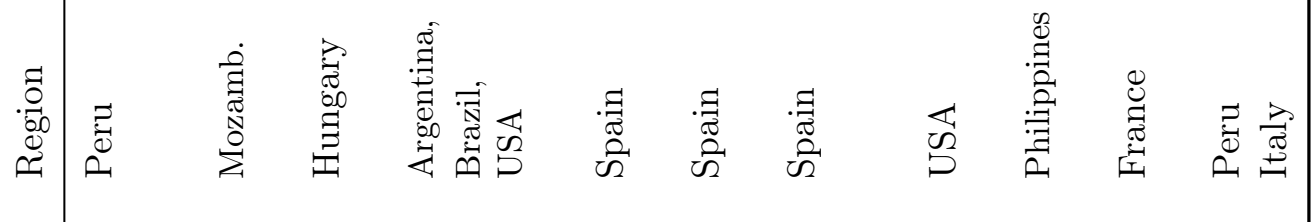

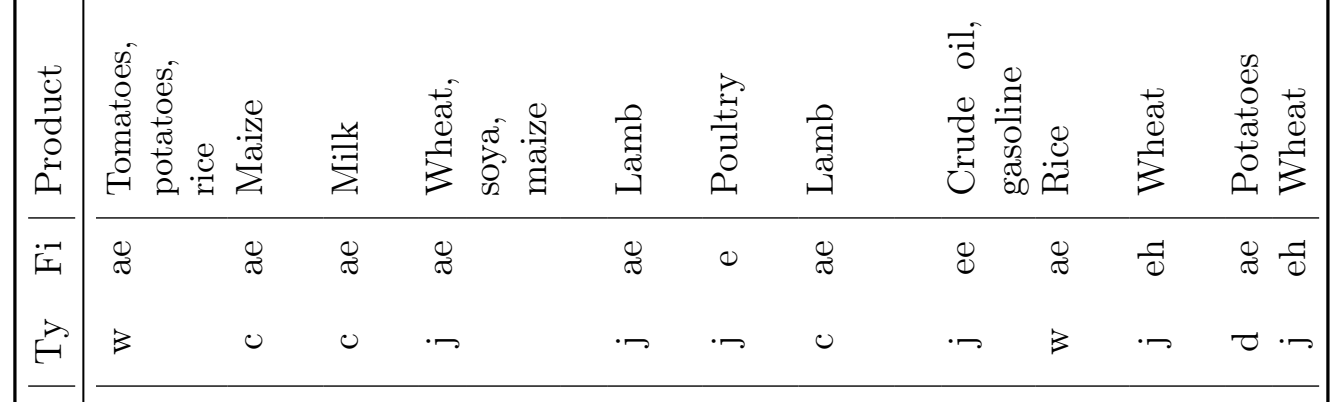

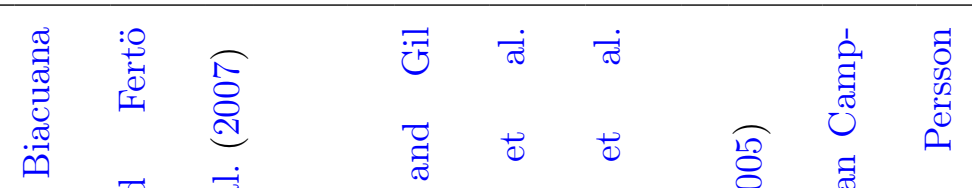

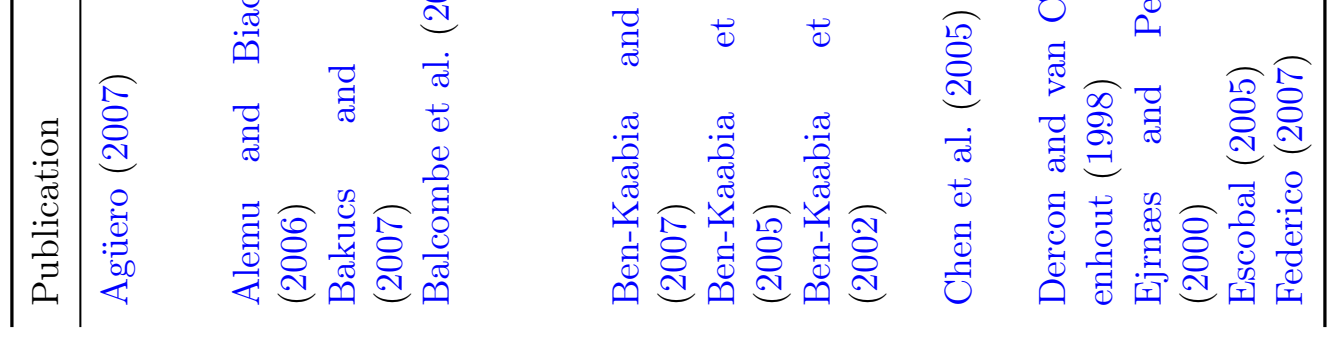




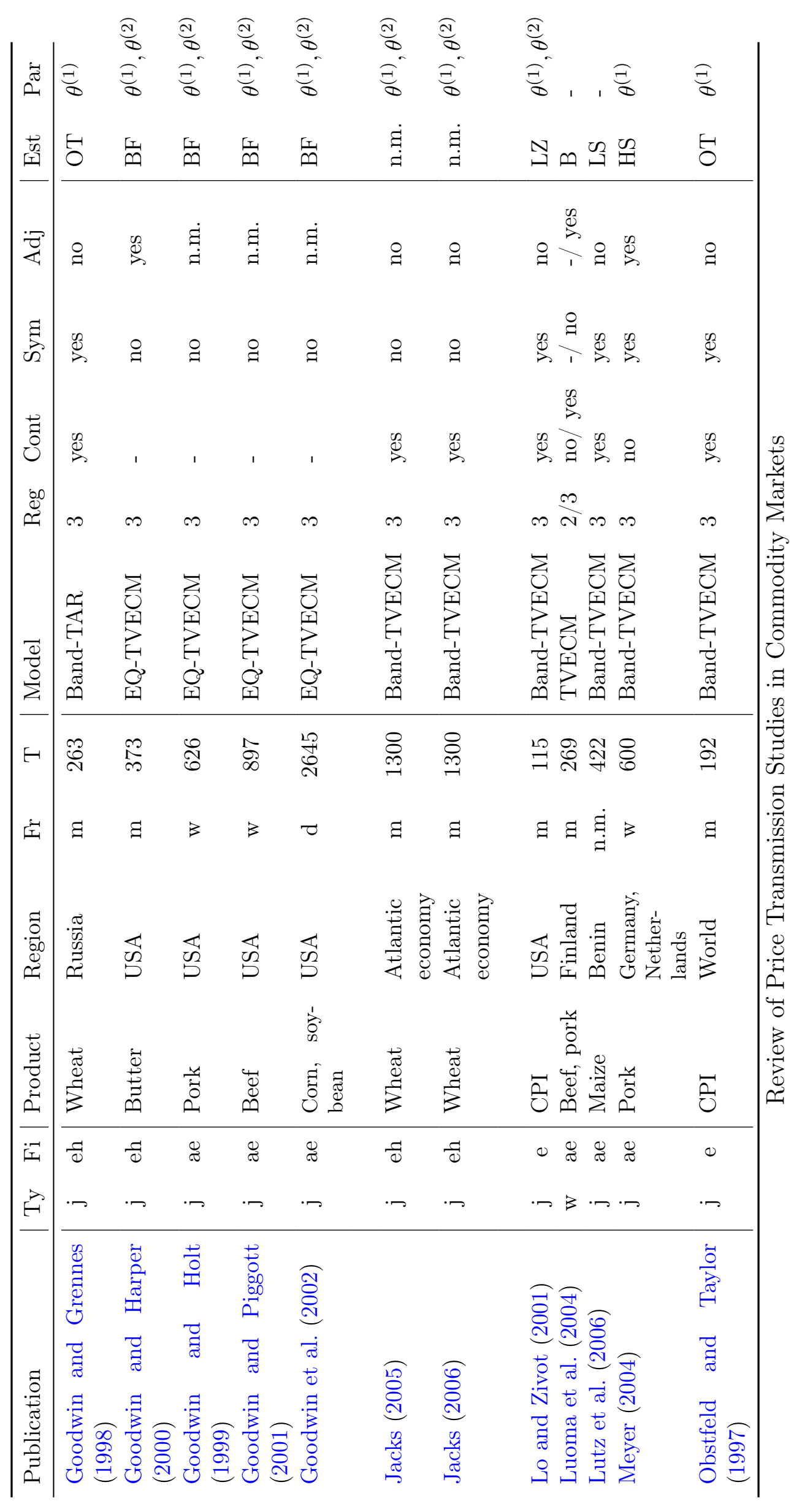




\begin{tabular}{|c|c|c|c|c|c|c|c|c|c|c|c|c|c|}
\hline Publication & Ty & $\mathrm{Fi}$ & Product & Region & Fr & $\mathrm{T}$ & Model & Reg & Cont & Sym & Adj & Est & Par \\
\hline $\begin{array}{l}\text { O'Connel and Wei } \\
(2002)\end{array}$ & $\mathrm{j}$ & $\mathrm{e}$ & $\mathrm{CPI}$ & USA & $q$ & $42-68$ & TAR & 2 & - & - & - & n.m. & $\overline{\theta^{(1)}, d}$ \\
\hline Park et al. (2007) & $\mathrm{j}$ & ee & $\begin{array}{l}\text { Natural } \\
\text { gas }\end{array}$ & $\begin{array}{l}\text { Canada, } \\
\text { USA }\end{array}$ & $\mathrm{d}$ & 1290 & Band-TVECM & 3 & no & no & yes & $\mathrm{LZ}$ & $\begin{array}{l}\theta^{(1)}, \theta^{(2)}, \\
d\end{array}$ \\
\hline $\begin{array}{l}\text { Pede and McKenzie } \\
(2005)\end{array}$ & $\mathrm{c}$ & ae & Maize & Benin & $\mathrm{w}$ & 162 & TVECM & 2 & - & - & - & HS & $\beta, \theta^{(1)}$ \\
\hline Sephton (2003) & j & ae & $\begin{array}{l}\text { Corn, soy- } \\
\text { bean }\end{array}$ & USA & $\mathrm{d}$ & 2645 & TVECM & 2 & - & - & - & HS & $\beta, \theta^{(1)}$ \\
\hline $\begin{array}{l}\text { Serra and Goodwin } \\
(2003)\end{array}$ & $\mathrm{j}$ & ae & Dairy & Spain & $\mathrm{m}$ & 78 & EQ-TVECM & 3 & - & no & yes & $\mathrm{LS}$ & $\theta^{(1)}, \theta^{(2)}$ \\
\hline $\begin{array}{l}\text { Serra and Goodwin } \\
(2004)\end{array}$ & $\mathrm{j}$ & ae & Eggs & USA & $\mathrm{m}$ & 372 & TVECM & $2 / 3$ & $-/$ n.m. & $-/$ n.m. & $-/$ n.m. & HS & $\begin{array}{l}\theta^{(1)} / \theta^{(1)} \\
\theta^{(2)}\end{array}$ \\
\hline Serra et al. (2006a) & j & ae & Pork & $\begin{array}{l}\text { Denmark, } \\
\text { France, } \\
\text { Germany, } \\
\text { Spain }\end{array}$ & $\mathrm{w}$ & 574 & EQ-TAR & 3 & - & no & yes & $\mathrm{BF}$ & $\theta^{(1)}, \theta^{(2)}$ \\
\hline Serra et al. (2006b) & j & ae & Eggs & USA & $\mathrm{m}$ & 360 & EQ-TAR & 3 & - & no & yes & n.m. & $\theta^{(1)}, \theta^{(2)}$ \\
\hline $\begin{array}{l}\text { Trenkler and Wolf } \\
(2005)\end{array}$ & $\mathrm{j}$ & eh & $\begin{array}{l}\text { Wheat } \\
\text { flour }\end{array}$ & Poland & $\mathrm{m}$ & 190 & Band-TAR & 3 & yes & yes & no & LZ & n.m. \\
\hline Uchezuba (2005) & $\mathrm{m}$ & ae & Apples & $\begin{array}{l}\text { South } \\
\text { Africa }\end{array}$ & $\mathrm{m}$ & 148 & Band-TVECM & 3 & no & no & yes & $\mathrm{LZ}$ & $\theta^{(1)}, \theta^{(2)}$ \\
\hline van Campenhout (2007) & $\mathrm{j}$ & ae & Maize & Tanzania & $\mathrm{w}$ & 626 & EQ-TAR & 3 & - & yes & no & n.m. & $\theta^{(1)}, \theta^{(2)}$ \\
\hline
\end{tabular}

Table DI.5: Review of Price Transmission Studies in Commodity Markets 


\section{Appendix DII: Simulation Study}

We perform a simulation study in order to assess the performance of the estimation methods used almost exclusively in applied research, namely sequential conditional least squares (SCLS) in the case of the TVECM and the Expectation-MaximizationAlgorithm (EMA) in the case of the MSVECM. We generate 1000 replications of two prices $p_{t}^{A}$ and $p_{t}^{B}$ per dataset, which follow a certain TVECM-specification and MSVECM-specification respectively.

Each DGP is assumed to consist of $l+1=M=3$ regimes, in one of which no error correction takes place, i.e. where the two prices are not cointegrated. ${ }^{46}$ Since the equilibrium errors are corrected toward the long-run relationship itself and not toward a band around it in the case of the MSVECM, we assume the error correction process of the TVECM to be of equilibrium-type in order to ensure comparability of the adjustment processes, that is, the equilibrium errors of the threshold model are also assumed to be corrected toward the long-run relationship itself, which means that we assume $\boldsymbol{\mu}^{(j)}=0$ in (D.11), p. 172. Furthermore, we assume the short-run dynamics $\Psi_{i}^{(j)}=0$ for the sake of simplicity. Hence, the DGP corresponds to the simple nonlinear VECM as outlined in Balke and Fomby (1997, p. 629):

$$
\begin{aligned}
\Delta \boldsymbol{p}_{t} & =\boldsymbol{\alpha}^{\left(J_{t}\right)} e c t_{t-1}+\epsilon_{t} \\
=\left(\begin{array}{c}
\Delta p_{t}^{A} \\
\Delta p_{t}^{B}
\end{array}\right) & =\left(\begin{array}{l}
\alpha^{A\left(J_{t}\right)} \\
\alpha^{B\left(J_{t}\right)}
\end{array}\right) e c t_{t-1}+\left(\begin{array}{c}
\epsilon_{t}^{A} \\
\epsilon_{t}^{B}
\end{array}\right) .
\end{aligned}
$$

Each dataset is generated containing $t=1, \ldots, T ; T=150,500,1500$ time points respectively. ${ }^{47}$ The lengths $T$ are based on the time series used in the studies of the literature review in Table DI.5. The first two values of $T$ denote a short and a long time series as typically used in empirical research. They roughly correspond to the first (162 measurements) and the third quartile (564 measurements) of the lengths of the time series of all studies except the five publications which use daily data. Very long time series of $T=1500$ observations will typically rarely be available in PT analysis. They correspond roughly to the mean length (1560 measurements) of the time series of daily observations used in Escobal (2005), Park et al. (2007) and Agüero (2007); the datasets of Goodwin et al. (2002) and Sephton (2003) with 2645 daily observations are not regarded since they are exceptionally long.

In particular, we assume the parameter of the cointegration relationship and the com-

\footnotetext{
${ }^{46}$ In the case of the TVECM, it is the middle regime that does not show error correction, i.e., $\boldsymbol{\alpha}^{(2)}=0$; for the MSVECM, it is the first, i.e., $\boldsymbol{\alpha}^{(1)}=0$.

${ }^{47}$ We actually generate $T+200$ observations of each dataset and throw away the first 200 in order to reduce the influence of the starting value.
} 
mon trend to $\beta=-1$ and $\phi=1$ respectively. We generate the equilibrium error process $\left\{e c t_{t}\right\}$ and the common stochastic trend $\left\{B_{t}\right\}$ according to

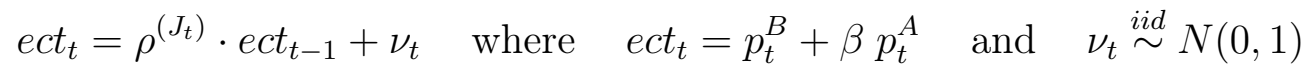

$$
\begin{aligned}
& B_{t}=B_{t-1}+\eta_{t} \quad \text { where } \quad B_{t}=p_{t}^{B}+\phi p_{t}^{A} \quad \text { and } \quad \eta_{t} \stackrel{i i d}{\sim} N(0,1)
\end{aligned}
$$

The corresponding error correction parameters $\boldsymbol{\alpha}^{(j)}$ and the price series $p_{t}^{A}, p_{t}^{B}$ in (DII.1) may then be calculated based on the generated values of ect $t_{t}$ and $B_{t}$ via the identities

$$
\begin{array}{rlrl}
p_{t}^{A} & =\frac{B_{t}-e c t_{t}}{\phi-\beta} \\
\alpha^{A(j)} & =\frac{1-\rho^{(j)}}{\phi-\beta} & p_{t}^{B} & =\frac{\phi \cdot e c t_{t}-\beta B_{t}}{\phi-\beta} \\
\alpha^{B(j)} & =-\frac{\phi\left(1-\rho^{(j)}\right)}{\phi-\beta} .
\end{array}
$$

We generate datasets for the four cases according to Table DII.1. In cases I and IV, we are interested in the performance of the estimation methods depending on the true parameters introducing nonlinearity, i.e., the thresholds and the transition matrix. ${ }^{48}$ Hence, we focus on the estimation of the thresholds and the ergodic probabilities depending on a set of varying true thresholds and ergodic probabilities incorporating the error correction parameter as a nuisance parameter in order to study whether and to what extent they influence the estimation results. ${ }^{49}$ The parameters varied in the DGP of the TVECM are the thresholds $\theta^{(1)}, \theta^{(2)}$ and the error correction parameters $\rho^{(1)}, \rho^{(3)}$ of the outer regimes. Regarding the DGP of the MSVECM, the ergodic probabilities $\pi^{(2)}, \pi^{(3)}$ and the error correction parameters $\rho^{(1)}, \rho^{(3)}$ are varied.$^{50}$

\footnotetext{
${ }^{48}$ Since the transition matrix of the MSVECM-DGP considered contains nine elements, we use the three corresponding ergodic probabilities $\boldsymbol{\pi}=\left(\pi^{(1)} \pi^{(2)} \pi^{(3)}\right)^{\top}$ instead. These are, technically spoken, the normalized eigenvector of the matrix associated with its unit eigenvalue (cf. Hamilton, 1994, pp. 681). The transition matrix and the ergodic probabilities are connected via $\boldsymbol{\Gamma}=\boldsymbol{T} \boldsymbol{\Lambda} \boldsymbol{T}^{-1}$ (Hamilton, 1994, p. 730) where $\boldsymbol{T}$ is the $M \times M$ matrix of the eigenvectors and $\boldsymbol{\Lambda}$ the $M \times M$ diagonal matrix of the eigenvalues of $\boldsymbol{\Gamma}$. Hence, by holding all elements of $\boldsymbol{\Lambda}$ constant and only plugging in varying $\boldsymbol{\pi}$ in the column of $\boldsymbol{T}$ associated with the unit eigenvalue, the corresponding transition matrices can be obtained. The ergodic probabilities corresponding to an estimated transition matrix $\hat{\boldsymbol{\Gamma}}$ can be obtained, in turn, by the eigenvalue decomposition of the latter and normalization of the respective eigenvector. We obtained $\boldsymbol{\Lambda}$ and $\boldsymbol{T}$ from the eigenvalue decomposition of $\boldsymbol{\Gamma}=\left(\begin{array}{ccc}0.95 & 0.03 & 0.02 \\ 0.15 & 0.8 & 0.05 \\ 0.2 & 0.1 & 0.7\end{array}\right)$ since it implies ergodic probabilities of $\boldsymbol{\pi}=\left(\begin{array}{lll}0.769 & 0.154 & 0.077\end{array}\right)^{\top}$, i.e., it assigns quite distinct unconditional probabilities to the three regimes.

${ }^{49}$ Since we simulate according to (DII.2) and (DII.3), we vary the autoregressive parameter $\rho^{(j)}$ in (DII.2) instead of $\boldsymbol{\alpha}^{(j)}$.

${ }^{50}$ Note that $\boldsymbol{\alpha}^{(2)}=0$, implying that $\rho^{(2)}=1$ in both cases and $\pi^{(1)}=1-\left(\pi^{(2)}+\pi^{(3)}\right)$. As mentioned before, the ergodic probabilities denote the expected unconditional probabilities $\pi^{(j)}$ of the $j=1, \ldots, M ; M=3$ regimes. The frequencies of the regimes generated by a MSVECM-DGP should hence equal them asymptotically.
} 
Table DII.1: Design of the Simulation Study

\begin{tabular}{l|cc}
\hline Estimation & $\begin{array}{l}\text { DGP } \\
\text { TVECM }\end{array}$ & MSVECM \\
\hline SCLS & I & II \\
EMA & III & IV \\
\hline
\end{tabular}

As criterion for measuring the performance of the estimation of the thresholds via SCLS and the ergodic probabilities via EMA, we choose the mean squared error $\operatorname{MSE}_{\hat{\lambda}}=\mathrm{E}(\hat{\lambda}-\lambda)^{2}=[\operatorname{Bias}(\hat{\lambda})]^{2}+\sigma^{2}(\hat{\lambda})$. Hence, the MSE of SCLS is estimated for $\hat{\lambda} \in\left\{\hat{\theta}^{(1)}, \hat{\theta}^{(2)}\right\}$ and the MSE of the EMA for $\hat{\lambda} \in\left\{, \hat{\pi}^{(1)}, \hat{\pi}^{(2)}, \hat{\pi}^{(3)}\right\}$. We approximate the functional relationship between the MSE and the varying true parameters of the DGPs by $\widehat{\operatorname{MSE}_{\hat{\lambda}}}=[\widehat{\operatorname{Bias}(\hat{\lambda})}]^{2}+\widehat{\sigma^{2}(\hat{\lambda})}$. The evaluation of

$$
\operatorname{MSE}_{\hat{\lambda}}^{S C L S}=f\left(\theta^{(1)}, \theta^{(2)}, \rho^{(1)}, \rho^{(3)}\right) \text { and } \operatorname{MSE}_{\hat{\lambda}}^{E M A}=f\left(\pi^{(2)}, \pi^{(3)}, \rho^{(1)}, \rho^{(3)}\right)
$$

for grids of true parameters is first computationally very demanding and can hardly be illustrated graphically due to its five dimensions. We thus reduce the number of dimensions to three by evaluating the MSE function by holding its third and fourth parameters constant $\left.\mathrm{MSE}_{\hat{\lambda}}^{A}=f\left(\theta^{(1)}, \theta^{(2)} \mid \rho^{(1)}, \rho^{(3)}\right), \mathrm{MSE}_{\hat{\lambda}}^{C}=f\left(\pi^{(2)}, \pi^{(3)} \mid \rho^{(1)}, \rho^{(3)}\right)\right)$ and evaluating it by holding its first and second parameters constant $\left(\mathrm{MSE}_{\hat{\lambda}}^{B}=f\left(\rho^{(1)}, \rho^{(3)} \mid \theta^{(1)}, \theta^{(2)}\right)\right.$, $\left.\operatorname{MSE}_{\hat{\lambda}}^{D}=f\left(\rho^{(1)}, \rho^{(3)} \mid \pi^{(2)}, \pi^{(3)}\right)\right)$ respectively. Although we restrict the simulation on these two subspaces, detailed insights into the behavior of the estimation methods can still be expected.

In cases II and III it is not possible to estimate the bias by simulation because there is no correspondence between the estimated thresholds and the ergodic probabilities, which served for the artificial generation of the data, and vice versa. We therefore try to assess for each observation whether its regime was correctly classified by the estimation method. However, this kind of inference has two identification problems in the case of the EMA. First, the method only allows probabilistic statements regarding the regime incidences. The approach of considering the regime with the highest smoothed probability at some time $t$ as the one that most likely occurred may, of course, randomly lead to incorrect identification. Second, Markov-switching models in general suffer from the problem regime identification regarding the transition matrix. This means that for repeated estimations, the numbering of the regimes may change so that the first regime of the first estimation needs not to be identical to the first one of the second estimation. One can try to identify the regimes by the magnitudes of their estimated parameters; however, a considerable amount of uncertainty remains, since the estimates vary randomly so that one can indeed obtain a wrong reordering. 
First, we generate TVECM datasets of the 16 combinations of $\theta^{(1)}=-0.5,-1,-2,-3$ and $\theta^{(2)}=0.5,1,2,3$ so that

$$
\operatorname{MSE}_{\hat{\lambda}}^{A}=f\left(M^{A} \mid \rho^{(1)}=0.9, \rho^{(3)}=0.9\right)
$$

where

$$
M^{A}=\left(\begin{array}{cccc}
-0.5,0.5 & -1,0.5 & -2,0.5 & -3,0.5 \\
-0.5,1 & -1,1 & -2,1 & -3,1 \\
-0.5,2 & -1,2 & -2,2 & -3,2 \\
-0.5,3 & -1,3 & -2,3 & -3,3
\end{array}\right)
$$

and the MSE is evaluated for each combination, i.e., for each element of $M^{A}$ individually.

Second, we generate TVECM datasets of the 16 combinations of $\rho^{(1)}=0.98,0.9,0.8,0.5$ and $\rho^{(3)}=0.98,0.9,0.8,0.5$ so that

$$
\operatorname{MSE}_{\hat{\lambda}}^{B}=f\left(M^{B} \mid \theta^{(1)}=-1, \theta^{(2)}=1\right)
$$

where

$$
M^{B}=\left(\begin{array}{cccc}
0.98,0.98 & 0.9,0.98 & 0.8,0.98 & 0.5,0.98 \\
0.98,0.9 & 0.9,0.9 & 0.8,0.9 & 0.5,0.9 \\
0.98,0.8 & 0.9,0.8 & 0.8,0.8 & 0.5,0.8 \\
0.98,0.5 & 0.9,0.5 & 0.8,0.5 & 0.5,0.5
\end{array}\right)
$$

For $\mathrm{T}=1500$, we only generate 9 combinations of $\theta^{(1)}=-0.5,-1,-2, \theta^{(2)}=0.5,1,2$ and $\rho^{(1)}=0.98,0.9,0.5, \rho^{(3)}=0.98,0.9,0.5$, respectively, due to the high computational cost involved for such long time series.

Case II

We generate one MSVECM dataset with

$$
\boldsymbol{\Gamma}=\left(\begin{array}{ccc}
0.8 & 0.15 & 0.05 \\
0.03 & 0.95 & 0.02 \\
0.1 & 0.2 & 0.7
\end{array}\right) \text { and } \rho^{(1)}=0.9, \rho^{(3)}=0.5
$$

Case III

We generate one TVECM dataset with $\theta^{(1)}=-1$ and $\theta^{(2)}=1$ and $\rho^{(1)}=0.9$, $\rho^{(3)}=$ 0.5 . 
First, we generate MSVECM datasets of the 16 combinations of $\pi^{(2)}=\frac{1}{12}, \frac{1}{6}, \frac{1}{4}, \frac{1}{3}$ and $\pi^{(3)}=\frac{1}{12}, \frac{1}{6}, \frac{1}{4}, \frac{1}{3}$ so that

$$
\operatorname{MSE}_{\hat{\lambda}}^{C}=f\left(M^{C} \mid \rho^{(2)}=0.9, \rho^{(3)}=0.5\right)
$$

where

$$
M^{C}=\left(\begin{array}{cccc}
0.08,0.08 & 0.17,0.08 & 0.25,0.08 & 0.33,0.08 \\
0.08,0.17 & 0.17,0.17 & 0.25,0.17 & 0.33,0.17 \\
0.08,0.25 & 0.17,0.25 & 0.25,0.25 & 0.33,0.25 \\
0.08,0.33 & 0.17,0.33 & 0.25,0.33 & 0.33,0.33
\end{array}\right) .
$$

Second, we generated MSVECM datasets of the 16 combinations of

$$
\rho^{(2)}=0.98,0.9,0.8,0.5 \text { and } \rho^{(3)}=0.98,0.9,0.8,0.5
$$

so that

$$
\operatorname{MSE}_{\hat{\lambda}}^{D}=f\left(M^{D} \mid \pi^{(2)}=\frac{1}{6}, \pi^{(3)}=\frac{1}{12}\right)
$$

where

$$
M^{D}=\left(\begin{array}{cccc}
0.98,0.98 & 0.9,0.98 & 0.8,0.98 & 0.5,0.98 \\
0.98,0.9 & 0.9,0.9 & 0.8,0.9 & 0.5,0.9 \\
0.98,0.8 & 0.9,0.8 & 0.8,0.8 & 0.5,0.8 \\
0.98,0.5 & 0.9,0.5 & 0.8,0.5 & 0.5,0.5
\end{array}\right)
$$

For $\mathrm{T}=1500$, we only generate 9 combinations of $\pi^{(2)}=\frac{1}{12}, \frac{1}{6}, \frac{1}{3}, \pi^{(3)}=\frac{1}{12}, \frac{1}{6}, \frac{1}{3}$ and $\rho^{(2)}=0.98,0.9,0.5, \rho^{(3)}=0.98,0.9,0.5$, respectively, due to the high computational cost involved for such long time series. We do not present all results in this paper. Further results may be obtained from the authors. 





\section{Curriculum Vitae}

1979

$1998-2000$

$2001-2003$

$2003-2004$

$2004-2005$

$2005-2010$
Born in Zschopau/Erzgebirge, German Democratic Republic

Studies of Agriculture (Grundstudium)

Martin-Luther-Universität Halle-Wittenberg

B.Sc. in Agricultural Economics

Georg-August-Universität Göttingen

M.Sc. in Agricultural Economics

Georg-August-Universität Göttingen

Studies of Arabic language

Sana'a, Yemen, and Cairo, Egypt

Ph.D. in Agricultural Economics

Georg-August-Universität Göttingen 

